\title{
Optimization of Piezoelectric Actuator Configuration on a Flexible Fin for Vibration Control Using Genetic Algorithms
}

Andrew Rader, B.Eng.

\author{
A Thesis submitted to \\ the Faculty of Graduate Studies and Research \\ in partial fulfilment of \\ the requirements for the degree of \\ Master of Applied Science \\ Ottawa-Carleton Institute for \\ Mechanical and Aerospace Engineering \\ Department of Mechanical and Aerospace Engineering \\ Carleton University \\ Ottawa, Ontario, Canada
}

July 2005

Copyright (c)

2005 - Andrew Rader, B.Eng. 


$\begin{array}{ll}\begin{array}{l}\text { Library and } \\ \text { Archives Canada }\end{array} & \begin{array}{l}\text { Bibliothèque et } \\ \text { Archives Canada }\end{array} \\ \begin{array}{l}\text { Published Heritage } \\ \text { Branch }\end{array} & \begin{array}{l}\text { Direction du } \\ \text { Patrimoine de l'édition }\end{array} \\ \begin{array}{l}\text { 395 Wellington Street } \\ \text { Ottawa ON K1A ON4 }\end{array} & \begin{array}{l}\text { 395, rue Wellington } \\ \text { Ottawa ON K1A ON4 } \\ \text { Canada }\end{array}\end{array}$

Your file Votre référence

ISBN: 0-494-10150-4

Ourfile Notre référence

ISBN: 0-494-10150-4

NOTICE:

The author has granted a nonexclusive license allowing Library and Archives Canada to reproduce, publish, archive, preserve, conserve, communicate to the public by telecommunication or on the Internet, loan, distribute and sell theses worldwide, for commercial or noncommercial purposes, in microform, paper, electronic and/or any other formats.

The author retains copyright ownership and moral rights in this thesis. Neither the thesis nor substantial extracts from it may be printed or otherwise reproduced without the author's permission.
AVIS:

L'auteur a accordé une licence non exclusive permettant à la Bibliothèque et Archives Canada de reproduire, publier, archiver, sauvegarder, conserver, transmettre au public par télécommunication ou par l'Internet, prêter, distribuer et vendre des thèses partout dans le monde, à des fins commerciales ou autres, sur support microforme, papier, électronique et/ou autres formats.

L'auteur conserve la propriété du droit d'auteur et des droits moraux qui protège cette thèse. $\mathrm{Ni}$ la thèse ni des extraits substantiels de celle-ci ne doivent être imprimés ou autrement reproduits sans son autorisation.
In compliance with the Canadian

Privacy Act some supporting forms may have been removed from this thesis.

While these forms may be included in the document page count, their removal does not represent any loss of content from the thesis.
Conformément à la loi canadienne sur la protection de la vie privée, quelques formulaires secondaires ont été enlevés de cette thèse.

Bien que ces formulaires aient inclus dans la pagination, il n'y aura aucun contenu manquant.

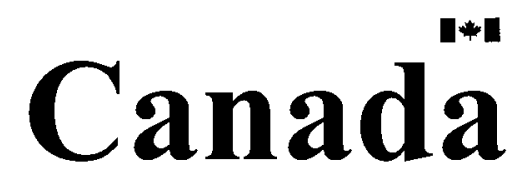




\begin{abstract}
An efficient solution to slow crack growth caused by buffet-induced vibration of vertical tails on twin-tail fighter airplanes is to use piezoelectric actuators and sensors to create an adaptive structure that actively reduces the buffeting. The optimization of the sensor and actuator configuration is critical to ensure that the structure will be effective in reducing the vibration for the anticipated loading conditions.

This thesis uses genetic algorithms to solve two optimization problems for vibration control of the first three modes of the fin. The first problem optimizes the position of a single pair of piezoelectric actuators on the fin. The second problem optimizes the activation of a pre-determined number of actuator pairs. The fitness functions for optimization are determined from the Frequency Response Functions (FRFs) measured at an accelerometer for the activation of each possible actuator. Individual modal and multi-modal acceleration and displacement vibration control are considered. The advantage of this method lies in the decoupling of the fitness function formulation from the optimization. In comparison to previous approaches, this allows optimization on much more complex geometries where the derivation of an analytical fitness function is prohibitive or impossible.

The optimization results obtained through simulation are verified through a comparison with results obtained from an experimental model of the fin. The agreement between results from simulation and experiment demonstrates the validity of the optimizations.
\end{abstract}




\section{Acknowledgments}

I would like to thank Professor Fred Afagh and Dr. Aghil Yousefi-Koma for all their insight and time. They allowed me the freedom to work independently and pursue my own research, but were always available when I needed their experience, support, or when I needed additional motivation to keep me on track. This thesis could not have been completed without the assistance provided by Professor Afagh in particular.

I am grateful to the National Research Council of Canada, and specifically to everyone at the Aeroacoustics and Structural Dynamics, Structures, Materials, and Propulsion (SDSMP) laboratory of the Institute for Aerospace Research (IAR). Dr. Yousefi-Koma and Eric Chen constructed the experimental model of the fin and assisted greatly in gathering the experimental results. Dr. Yousefi-Koma and Dr. David Zimcik at the SDSMP laboratory contributed in writing the journal and conference papers prepared as part of this research, as did Professor Afagh at Carleton University. A conversation with Professor David Amundsen in the School of Mathematics and Statistics at Carleton University was instrumental in the development of the surface fitting genetic algorithm.

I would like to acknowledge the generous financial support of the Natural Sciences and Engineering Research Council (NSERC), and the funding provided for the project by the Canadian Department of National Defence (DND). I also would like to thank my family and Helena Forbes for their unconditional support. 


\section{List of Symbols}

\begin{tabular}{ll}
$F R F$ & Frequency Response Function \\
$x, y$ & Cartesian co-ordinate system \\
$\varepsilon$ & Strain \\
$d$ & Piezoelectric charge constant \\
$t$ & Material thickness \\
$V$ & Applied voltage \\
$\varepsilon$ & Strain \\
$\alpha$ & Thermal expansion coefficient \\
$T$ & Temperature \\
$\mathbf{g}$ & Earth's gravitational constant $\left(9.81 \mathrm{~m} / \mathrm{s}^{2}\right)$ \\
$p$ & Polynomial order \\
$n$ & Number of coefficients in a polynomial \\
$z$ & Polynomial surface magnitude \\
$a$ & Polynomial surface coefficient \\
$d$ & Actual FRF magnitude \\
$r$ & Range parameter \\
$l$ & Size of population pool \\
$\beta$ & Number of individuals in a population \\
$\lambda$ & Random number between 0 and 1 \\
\hline & Random number between -1 and 1 \\
\hline &
\end{tabular}


$w_{1}, w_{2}, w_{3}$

$\omega_{1}, \omega_{2}, \omega_{3}$

$q$

$R M E$

$M E$
Modal weighting factors

Natural frequencies

Size of population for multiple actuator configuration optimization

Relative mean effectiveness

Minimum effectiveness 


\section{Table of Contents}

$\begin{array}{ll}\text { Abstract } & \text { ii }\end{array}$

Acknowledgments $\quad$ iii

List of Symbols $\quad$ iv

Table of Contents $\quad$ vi

List of Tables $\quad$ x

List of Figures $\quad$ xii

1 Introduction 1

1.1 Thesis Overview $\ldots \ldots \ldots \ldots \ldots \ldots$

1.2 Motivation ....................... 3

1.2.1 Active Tail Buffet Suppression Project . . . . . . . . . . . . . 4

1.2.2 Vibration Control of Individual Modes . . . . . . . . . . . . 7

1.2.3 Vibration Control of Multiple Modes . . . . . . . . . . . . 7

1.2.4 Acceleration vs. Displacement Control . . . . . . . . . . . . 7

1.3 Background .......................... 8

1.3.1 Active Materials . . . . . . . . . . . . . 8

1.3.2 Piezoelectric Actuators f . . . . . . . . . . . . 8

1.3.3 Adaptive Structures . . . . . . . . . . . . . . . . 9

1.3 .4 Genetic Algorithms . . . . . . . . . . . . . . . . 10

vi 
1.3.5 Combination of Finite Element Methods with Genetic Algorithms . 11

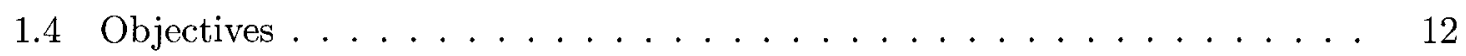

2 Flexible Fin Overview and Data Extraction 14

2.1 Finite Element Methods Overview . . . . . . . . . . . . . . . 14

2.2 Fin Configurations . . . . . . . . . . . . . . . 15

2.2.1 Experimental Set-up vs. Finite Element Model . . . . . . . . . 15

2.2 .2 Accelerometers . . . . . . . . . . . . . . 16

2.2.3 Finite Element Model for Actuator Configuration Optimization . . . 18

2.3 Finite Element Method Data Acquisition _ . . . . . . . . . . . . . . 19

2.3.1 FEM Elements and Configuration . . . . . . . . . . . . . . 20

2.3 .2 Thermal Analogy . . . . . . . . . . . . . . . 20

2.3.3 Patran and Frequency Response Simulation . . . . . . . . . . . 22

2.3.4 Nastran and Post Processing Results . . . . . . . . . . . . . 23

2.3.5 Excel Files and Selection of Peak FRF . . . . . . . . . . . . . . . 24

3 Optimization of the Position of a Single Pair of Actuators 26

3.1 Approach: Polynomial Surface Fitting to Obtain a Continuous Fitness Function 26

3.1.1 Suitability of Three-Dimensional Polynomial Surfaces . . . . . . . 27

3.1.2 Fitness Function for Polynomial Surface Fitting . . . . . . . . . 28

3.1.3 Convergence of Solution . . . . . . . . . . . . . . . . 30

3.2 Genetic Algorithm for Surface Fitting . . . . . . . . . . . . . . . 32

3.2 .1 Genetic Algorithm Steps . . . . . . . . . . . . . . . . . 34

3.2.2 Factors Affecting Convergence . . . . . . . . . . . . . . 44

3.3 Polynomial Surface Fitting Results . . . . . . . . . . . . . . . . 48

3.3.1 First Mode . . . . . . . . . . . . . . . . . . . 48

3.3 .2 Second Mode . . . . . . . . . . . . . . . . . . 48

3.3 .3 Third Mode . . . . . . . . . . . . . . . . . . . 51

3.4 Genetic Algorithm for Actuator Positional Optimization . . . . . . . . . 52

3.4.1 Fitness Function for Positional Optimization . . . . . . . . . . 53

vii 
3.4 .2 Genetic Algorithm Steps . . . . . . . . . . . . . . . 54

3.4 .3 Positional Optimization .................... 55

4 Multiple Actuator Grouping Optimization $\quad 61$

4.1 Approach: Direct Peak FRF Maximization . . . . . . . . . . . . . 61

4.2 Frequency Response Function Results . . . . . . . . . . . . . . . 62

4.2 .1 First Mode . . . . . . . . . . . . . . . . 62

4.2 .2 Second Mode . . . . . . . . . . . . . . . . . . 62

4.2 .3 Third Mode . . . . . . . . . . . . . . . 63

4.3 Genetic Algorithm for Grouping Optimization . . . . . . . . . . . . 64

4.3 .1 Fitness Function . . . . . . . . . . . . . . 65

4.3 .2 Genetic Algorithm Steps . . . . . . . . . . . . . . . . 67

4.4 Assessing Configurations . . . . . . . . . . . . . . . . 68

4.4 .1 Actuation Authority . . . . . . . . . . . . 68

4.4.2 Minimum Effectiveness (ME) and Relative Mean Effectiveness (RME) 69

4.5 Optimization Types $\ldots \ldots \ldots \ldots \ldots \ldots \ldots$

4.5 .1 Actuation Maximization . . . . . . . . . . . . . 70

4.5.2 Minimization of Number of Actuators . . . . . . . . . . 71

4.5 .3 Trade-off Study . . . . . . . . . . . . . . . . . . . 71

4.6 Configurations for Actuation Maximization . . . . . . . . . . . 72

4.6 .1 First Mode . . . . . . . . . . . . . . . 72

4.6 .2 Second Mode . . . . . . . . . . . . . . . . . 73

4.6 .3 Third Mode . . . . . . . . . . . . . . 75

4.6.4 Multi-Modal Acceleration . . . . . . . . . . . 76

4.6.5 Multi-Modal Displacement . . . . . . . . . . . 77

4.7 Example of a Trade-off Study . . . . . . . . . . . . . . . 80

5 Verification of Simulation through Experimentation $\quad 83$

5.1 Experimental Data Acquisition . . . . . . . . . . . . . 84

5.1 .1 Test Bed Specifications . . . . . . . . . . . 84

viii 
5.1 .2 Experimental Results . . . . . . . . . . . . 85

5.1 .3 Scaling of Experimental Results . . . . . . . . . . . . 86

5.2 Comparison of Experimental Results with Simulation . . . . . . . . . . 87

5.2.1 Comparison with Results from FEM model in Experimental Configuration $(12$ Actuators $) \ldots \ldots \ldots \ldots$

5.2.2 Comparison with Results from Multiple Actuator Configuration Optimization $($ Chapter 4$) \ldots \ldots \ldots \ldots$

5.2.3 Comparison with Results from Single Actuator Positional Optimization $($ Chapter 3$) \ldots \ldots \ldots \ldots$

6 Conclusions and Recommendations $\quad 94$

6.1 Conclusions . . . . . . . . . . . . . . . . . . . . . 94

6.2 Recommendations . . . . . . . . . . . . . . . 96

$\begin{array}{ll}\text { List of References } & 99\end{array}$

$\begin{array}{ll}\text { Appendix A Surface Fitting Matlab Code } & 102\end{array}$

Appendix B Single Actuator Matlab Optimization Code 144

$\begin{array}{ll}\text { Appendix C Grouping Optimization Matlab Code } & 168\end{array}$ 


\section{List of Tables}

2.1 X and Y Accelerometer Positions on the Smart Fin along with Corresponding Finite Element Node . . . . . . . . . . . . . . . . . 17

3.1 Number of Coefficients for a Polynomial Surface by Polynomial Order . . . 27

3.2 Values of the Range Parameters for Each Polynomial Order . . . . . . . . 36

3.3 Approximate Number of Generations per Second by Polynomial Order on a $2000 \mathrm{MHz}$ Pentium PC . . . . . . . . . . . . . . . . . 44

3.4 Effect of Polynomial Order on Error . . . . . . . . . . . . . . . 45

3.5 Effect of the Initial Population Pool Size on Convergence . . . . . . . . . 46

3.6 Effect of Mutation Probability on Convergence . . . . . . . . . . 47

3.7 Effect of Mutation Severity on Convergence . . . . . . . . . . . . . . 48

3.8 Best Single 9th Order Surface and Sub-surface Coordinates and Error for the Second Mode ... . . . . . . . . . . . . . . . . . 51

3.9 Optimum Actuator Position with Different Weighting Factors . . . . . . . 59

4.1 Example of a Forced Mutation (FM) Trigger During reproduction . . . . . 68

4.2 Selected Actuation Maximization Configurations for the First Mode . . . . 73

4.3 Selected Actuation Maximization Configurations for the Second Mode . . . 74

4.4 Selected Actuation Maximization Configurations for the Third Mode . . . $\quad 75$

4.5 Selected Actuation Maximization Configurations for the Multi-modal Acceleration Control . . . . . . . . . . . . . . . . . . . 77

4.6 Selected Actuation Maximization Configurations for the Multi-modal Displacement Control . . . . . . . . . . . . . . . 80

5.1 Comparison of Natural Frequencies from Simulation and Experimental Model 83 
5.2 Material Properties of Aluminum and Piezoelectric Material . . . . . . . . 85

5.3 Accelerometer Information for the Flexible Fin Test Bed . . . . . . . . . . 86

5.4 Comparison of Experimental and Simulation Results for the First Mode of the Smart Fin . . . . . . . . . . . . . . . . . . . 89

5.5 Comparison of Experimental and Scaled Simulation Results for the Second Mode of the Smart Fin . . . . . . . . . . . . . . . . 90

5.6 Comparison of Experimental and Scaled Simulation Results for the Third Mode of the Smart Fin . . . . . . . . . . . . . . . . . . . . 91

5.7 Actuator Ranking from Most Effective to Least For Individual Modal Control 92

5.8 Actuator Ranking from Most Effective to Least For Multi-Modal Control . 92

5.9 Comparison of Actuator Ranking of Multiple Actuator Optimization with Experimental Results for Individual Modal Control using Equivalent Actuators 93

5.10 Comparison of Actuator Ranking of Multiple Actuator Optimization with Experimental Results for Multi-Modal Control using Equivalent Actuators . 93 


\section{List of Figures}

1.1 Leading Edge Vortices of an F/A-18 at a High Angle of Attack [1] . . . . 4

1.2 F/A-18 Vertical Tail with Bonded Piezoelectric Actuators [2] . . . . . . 5

1.3 First Mode Dwell Reduction for Simulation (Left) and Experiment (Right) [3] 6

2.1 The Fin Mode Shapes . . . . . . . . . . . . . . . . . . . 15

2.2 Geometry of the Smart Fin in Experimental Configuration . . . . . . . 16

2.3 Finite Element Model of the Smart Fin in Experimental Configuration [4] . 17

2.4 Accelerometer Positions on the Smart Fin . . . . . . . . . . . . . . . 18

2.5 The 48 Actuator Positions from the Finite Element Model of the Smart Fin 19

2.6 Actuator Modelling using 25 HEX20 Solid Elements [4] . . . . . . . . 21

2.7 Example of Excel Results File for Extraction of Peak FRFs . . . . . . . . 25

2.8 Peak FRF at Accelerometer 1 with the Actuator at each of the 48 Possible

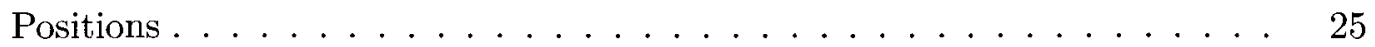

3.1 Typical SRSS Error (left) and Normalized Mean Error (right) vs. Generation Plots ................................... 31

3.2 Calculation of Range Parameter $\mathrm{r}_{0} \ldots \ldots \ldots \ldots \ldots$

3.3 Calculation of Range Parameter $r_{1} \ldots \ldots \ldots \ldots$

3.4 Calculation of Range Parameter $\mathrm{r}_{2} \ldots \ldots \ldots \ldots \ldots$

3.5 Example of Crossover at the Second Allele . . . . . . . . . . . . . . 40

3.6 Comparison of Convergence Rate With and Without the Immigration Operator 42

3.7 Comparison of the Discrete FRF Peak Magnitudes and the Best 3rd Order Polynomial Surface for the First Mode . . . . . . . . . . . . . . . . 49 
3.8 Comparison of the Discrete FRF Peak Magnitudes and the Best Single 9th Order Polynomial Surface for the Second Mode . . . . . . . . . . . . 50

3.9 The Actuator Test Area Subdivisions . . . . . . . . . . . . . . . 51

3.10 The First (a) and Second (b) Sub-surfaces for the Second Mode . . . . . . 52

3.11 The Third (a) and Fourth (b) Sub-surfaces for the Second Mode . . . . . 53

3.12 Comparison of the Discrete FRF Peak Magnitudes (a) and the Combined Subsurfaces (b) for the Second Mode . . . . . . . . . . . . . . . . 54

3.13 Comparison of the Discrete FRF Peak Magnitudes and the Best 8th Order Polynomial Surface for the Third Mode . . . . . . . . . . . . . . 55

3.14 The Optimum Actuator Pair Position for the First Mode $\ldots \ldots . . . .56$

3.15 The Optimum Actuator Pair Position for the Second Mode . . . . . . . . 57

3.16 The Optimum Actuator Pair Position for the Third Mode . . . . . . . . . 57

3.17 The Optimum Actuator Pair Position for Acceleration Control . . . . . . 58

3.18 The Optimum Actuator Pair Position for Displacement Control . . . . . . 59

3.19 Combined Multi-modal Polynomial with Weighting Factors to Equalize Mean FRF Magnitudes . . . . . . . . . . . . . . . . . . 60

4.1 Discrete FRF Peak Magnitudes for the First Mode . . . . . . . . . . . 63

4.2 Discrete FRF Peak Magnitudes for the Second Mode . . . . . . . . . 63

4.3 Discrete FRF Peak Magnitudes for the Third Mode . . . . . . . . . . 64

4.4 Actuator Numbering Scheme . . . . . . . . . . . . . . . . . . 72

4.5 Actuation Maximization Configurations for the First Mode . . . . . . . 73

4.6 Actuation Maximization Configurations for the Second Mode . . . . . . . 74

4.7 Actuation Maximization Configurations for the Third Mode . . . . . . . . 76

4.8 Minimum Effectiveness (ME) and Relative Mean Effectiveness (RME) versus Number of Actuators for Acceleration Control . . . . . . . . . . 78

4.9 Actuation Maximization Configurations for Multi-modal Acceleration Control 79

4.10 Actuation Maximization Configurations for Multi-modal Acceleration Control 79

4.11 Minimum Effectiveness (ME) and Relative Mean Effectiveness (RME) versus Number of Actuators for Displacement Control . . . . . . . . . . . . . . 81 
4.12 Actuation Maximization Configurations for Multi-modal Displacement Control 82

4.13 Actuation Maximization Configurations for Multi-modal Displacement Control 82

5.1 Flexible Fin with Piezoelectric Actuators [3] . . . . . . . . . . . . . . 84

5.2 The Experimental Test Bed $[3] \ldots \ldots \ldots \ldots \ldots$

5.3 A Typical FRF vs. Time Plot . . . . . . . . . . . . . 86 


\section{Chapter 1}

\section{Introduction}

Buffet-induced vertical tail vibration is a significant problem, particularly for twin-tail fighter jets which require frequent inspection to prevent catastrophic failure [1]. In order to reduce the crack growth rate and therefore reduce the reliance on inspection, one of two possible approaches is generally adopted. The first involves stiffening the structure, partially to reduce the severity of vibration, but primarily to strengthen the structure in order to reduce crack growth. The second, and potentially more efficient approach, is to use an adaptive structure to actively reduce the buffeting in order to reduce crack growth and increase the lifespan of a vertical tail fin. This thesis focuses on optimizing the configuration of piezoelectric actuators on a flexible vertical tail fin in order to actively reduce induced buffeting displacement and acceleration at any point on the fin.

\subsection{Thesis Overview}

Chapter 1 describes the active tail buffet suppression project, conducted in part at the National Research Council of Canada, which was the primary motivation for this research. An overview of the project is presented, and recent developments are expounded. Acceleration and displacement vibration control and the relative effects of each are described as they relate to a reference point on the fin. A background of active materials, active structures, genetic algorithms, and some combinations of finite element methods with genetic algorithms in current literature are presented. 
Chapter 2 describes, in detail, the different configurations of the finite element and experimental models of the flexible fin that were used in this work. The methodology used to extract the frequency response results in terms of the frequency response function (FRF) from the finite element model using PATRAN and NASTRAN is discussed in detail. The experimental setup and methodology for FRF extraction is described.

Chapter 3 describes the optimization of the position of a single pair of piezoelectric actuators on the fin by using a genetic algorithm to fit the modal FRFs onto continuous surfaces in order to obtain a continuous fitness function. The derivation of this fitness function is described in detail. A complete examination of all steps of the genetic algorithm is a primary focus of the chapter. Particular attention is paid to the initial population pool and the mutation operator developed in this study to particularly suit three dimensional polynomial surface fitting. Factors that affect convergence such as population pool size, mutation probability, and mutation severity are examined in order to determine the optimal parameters which are later used in the genetic algorithm. The results of polynomial surface fitting are then presented for each mode. The second mode FRF was not well suited for representation as a single polynomial surface, but this problem was overcome by dividing the examined area of the fin into four neighbouring subregions, each of which provided good representations. A second genetic algorithm that was used to determine the optimal position of the actuator is then discussed in detail. Finally, the optimum piezoelectric actuator position is determined for several vibration control schemes.

Chapter 4 describes the optimization of the configuration of up to 48 individual piezoelectric actuators on the fin by direct maximization of the FRF for either an individual mode or multi-modal acceleration or displacement control. The derivation of the fitness function is examined for all vibration control schemes. The different types of optimization possible in terms of trade-off studies, actuation maximization, and minimization of number of actuators through application of the methodology are highlighted. The genetic algorithm used for optimization of the actuator configuration is discussed in detail, with emphasis on reproduction and mutation, including forced mutation, a unique feature of the algorithm. The FRF results for the first three modes are presented, and key measures of optimization 
effectiveness, namely actuation authority, minimum effectiveness (ME), and relative mean effectiveness (RME) are defined. The optimized configurations for individual modal control and multi-modal acceleration and displacement control are presented, and an example of a trade-off study between actuator weight and actuation authority is presented.

Chapter 5 presents a verification of the finite element simulation results by comparison to optimization results obtained from an experimental model of the fin. The experimental results are presented along with a modal scaling used to correlate the results to the simulation results. Comparisons of the experimental results with simulation results obtained from the finite element model of the fin in experimental, single actuator position optimization, and multiple actuator optimization configurations are conducted.

Chapter 6 emphasizes the major contributions to advancing the state of knowledge in the field and conclusions that can be inferred from the research, and provides recommendations for future directions of research.

\subsection{Motivation}

This research was conducted at the National Research Council of Canada and Carleton University in order to complement a project to develop an adaptive control system to actively reduce buffet induced tail vibrations as part of a multinational research project. An active control system has been developed for the flexible fin presented in this thesis in the Aeroacoustics and Structural Dynamics, Structures, Materials, and Propulsion (SDSMP) laboratory of the Institute for Aerospace Research (IAR) at the Ottawa branch of the National Research of Canada [3]. This control system was developed using evenly distributed pairs of piezoelectric actuators such as in the experimental configuration presented in Chapter 5 in this thesis. The efficiency of such a control system can be vastly improved by instead optimizing the actuator configuration. The purpose of the research described in this thesis is to present a novel approach to such an optimization and investigate the possible problems associated with the methodology. 


\subsubsection{Active Tail Buffet Suppression Project}

Buffet induced tail vibrations such as those shown in Figure 1.1 occur on high performance twin-tail aircraft such as the F/A-18 and F-15 when unsteady pressures associated with separated flow, or vortices, excite the vibration modes of the vertical tail fin structural assemblies [1]. This is a significant problem, particularly for the F/A-18 aircraft operated by Canada, Australia, and the United States, which requires frequent inspection to prevent catastrophic failure caused by crack growth. There have been numerous studies on using an active control system in order to reduce the buffet loads on both scaled models and actual aircraft [5] [6] [7]. An alternative method of vibration control to piezoelectric actuators is to use active rudder control [8]. Results of studies using piezoelectric actuators for vibration control have shown that a $60 \%$ reduction of first mode bending amplitude can be acheived, resulting in a significant extension of the lifespan of a tail fin. Other studies have been conducted by Boeing, NASA Langley, the Air Force Research Laboratory (AFRL), and Daimler Chrysler, to highlight a few [9] [10].

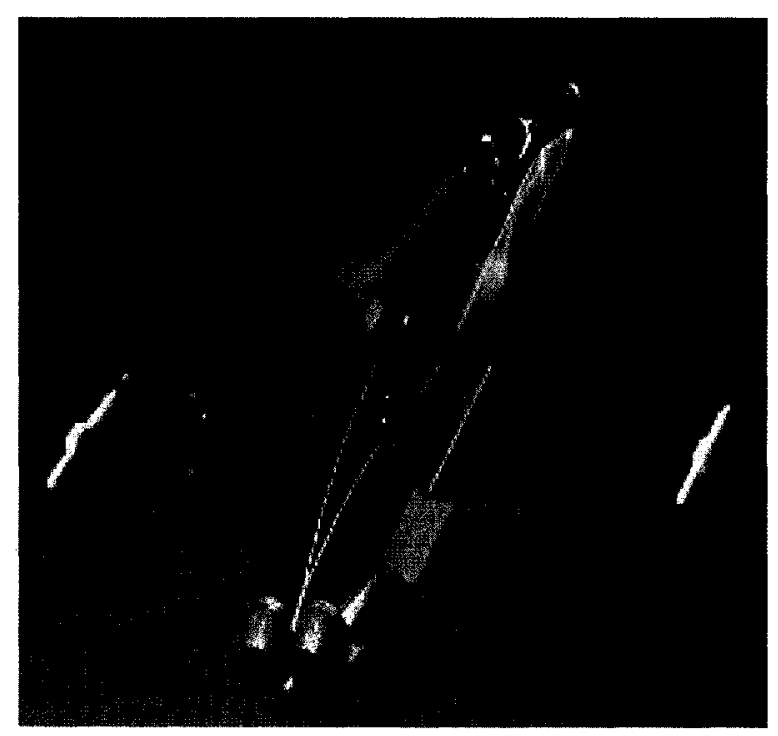

Figure 1.1: Leading Edge Vortices of an F/A-18 at a High Angle of Attack [1] 


\section{Project Overview}

A joint project between Canada, Australia, and the United States was initiated in 1997 under The Technical Cooperation Program (TTCP) to determine the suitability of an active control system to reduce the buffet induced tail vibrations on a full-scale F/A-18. In Canada, this involved the Canadian Department of National Defence (DND) and the National Research Council of Canada (NRC). The Canadian contribution was the development of an active control system using piezoelectric actuators [2]. The control scheme was able to provide a reduction of up to $60 \%$ in buffet vibration amplitude in the best case, and of $10 \%$ in the worst case [11]. If incorporated into the aircraft, this control system would double the durability of the fin. Figure 1.2 shows the full scale test of the control system with bonded piezoelectric actuators.

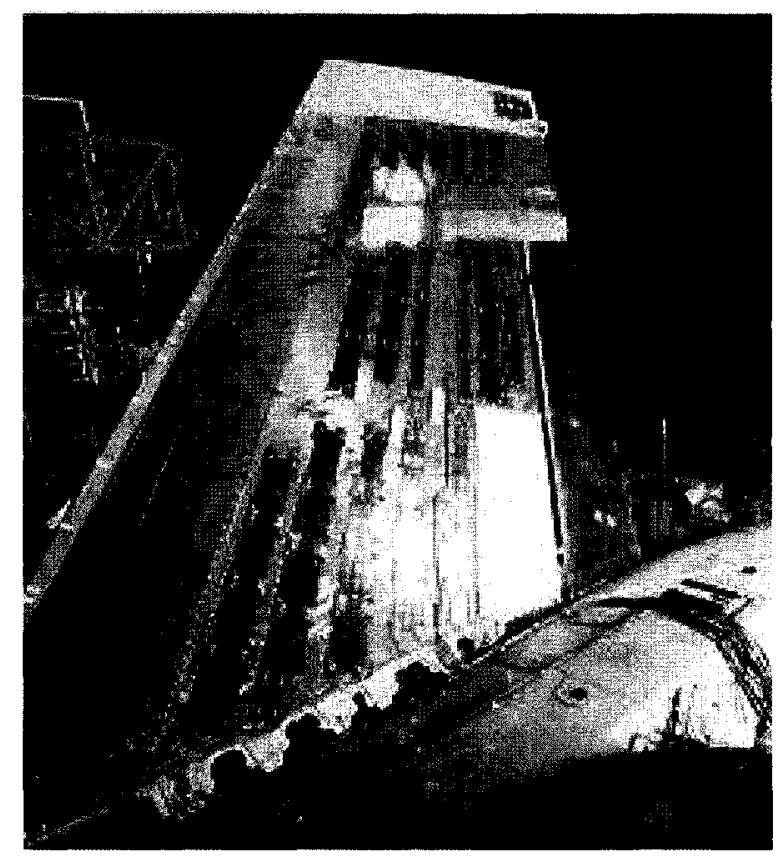

Figure 1.2: F/A-18 Vertical Tail with Bonded Piezoelectric Actuators [2]

\section{System Identification}

A primary challenge in the development of an active control system is system identification, or the extraction of a dynamic model for a flexible structure with piezoelectric transducers. 
Using a reduced model experimental flexible fin, a theoretical method of system identification using finite element modelling has been developed at the SDSMP laboratory at the NRC of Canada to model a dynamic system and to obtain its Frequency Response Function (FRF) [4]. A $\mu$ synthesis technique is then used to match a transfer function to the FRF plot. Experimental system identification was also carried out using the experimental test bed described in Chapter 5 of this thesis. A state space model produced the best fit to the experimental data, and resulted in good agreement between the two methods. This was an essential part of the development of a control system for the flexible fin.

\section{Control System}

A feedback control scheme for the experimental configuration of the flexible fin presented in this thesis using 12 pairs of actuators was developed at the SDSMP laboratory [3]. Using the $\mu$ synthesis technique, the transfer function of the system was derived from the FRF data. Based on this model, an active control system was implemented by using the RealTime Workshop and xPC TargetBox of MathWorks. Results demonstrated that active control systems could reduce the vibration significantly both in narrowband and broadband frequency ranges, as shown in Figure 1.3.

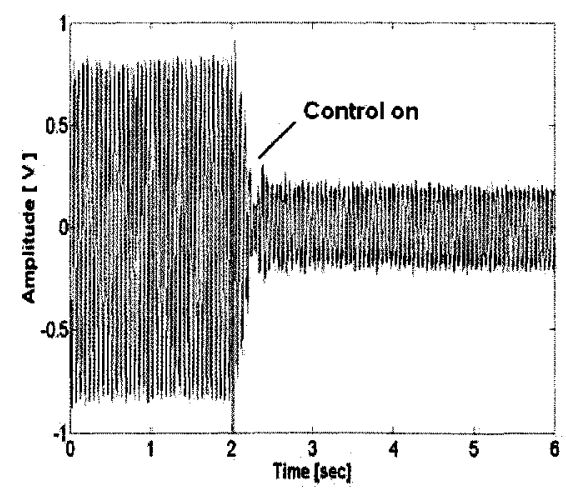

(a) Simulation

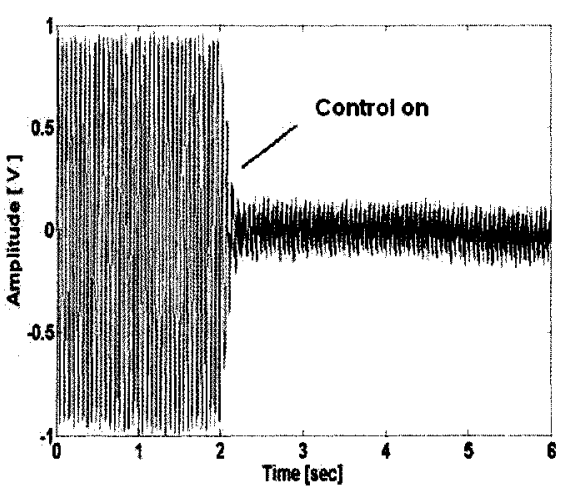

(b) Experiment

Figure 1.3: First Mode Dwell Reduction for Simulation (Left) and Experiment (Right) [3] 


\subsubsection{Vibration Control of Individual Modes}

In many operations, buffeting is only able to excite a particular vibrational mode of the vertical tail fin. In this case, it is efficient to design a control scheme to reduce the vibration of that mode, and to place actuators where they are best suited to control that mode. This results in the best possible position of actuators and most effective control system, but if unexpected frequencies are encountered, it may not be able to adequately control the resulting vibration. Alternatively, it is possible to distribute actuators over the fin. This would allow some reduction of any vibrations that could possibly be encountered, however efficiency would suffer, sometimes drastically, depending on the vibration mode.

\subsubsection{Vibration Control of Multiple Modes}

If it is not possible to identify a single mode of concern, but it is possible to identify a frequency range that is likely to be encountered in operation, it is possible to optimize an actuator configuration for multi-modal control. This entails identifying the corresponding vibration modes, and tailoring a system specifically to control either the acceleration or displacement (or a combination of each) for those modes. This results in a more versatile system, with a corresponding loss of actuation efficiency. It is, however, still much more efficient than a random or even distribution of actuators. It is inevitable that the wider the range of conditions a system is optimized for, the less effective the system is for each individual condition. Thus an understanding of the likely operational conditions for the system is critical.

\subsubsection{Acceleration vs. Displacement Control}

This research used both acceleration and displacement, measured with accelerometers, as independent bases for optimization of actuator configuration. Either can be important factors for vibration of the flexible fin. Buffet induced tail vibration is usually associated with activation of the first and second mode, and crack growth rate is primarily dictated by first mode vibrational displacement. However, the second and third modes are within 
the excitation range that is likely to be encountered in operation and are quite significant, particularly when considering acceleration.

\subsection{Background}

The research presented in this thesis falls into several fields, including active materials, piezoelectric actuators, flexible active structures, genetic algorithms, and the combination of a genetic algorithm with finite element methods.

\subsubsection{Active Materials}

Active materials incorporate physical properties that can be controlled by external stimulus, allowing them to be used in control systems where the material properties are selectively adjusted to achieve a specific purpose. Properties such as viscosity (in the case of magneto and electro-rheological fluids) or strains (in the case of magnetostrictive and electrostrictive materials or shape memory alloys) are typically controlled by electrical, magnetic, or temperature stimulus. Stimuli that can be rapidly adjusted such as an electric or magnetic field are particularly relevant, since they can be used in real time control systems.

\subsubsection{Piezoelectric Actuators}

Piezoelectrics are the most common form of electrostrictive materials that can generate an electric field when they are strained. As a result of this "direct piezoelectric effect" they can be used as sensors detecting straining of a host structure. Conversely, when subjected to an applied electric field, employing their "converse piezoelectric effect", these materials can be used as actuators to strain the host structure. As discussed later on in this thesis, both direct and converse piezoelectric effects are considered to be linearly proportional in the present study, i.e., the induced strain is assumed to be linearly proportional to the applied electric field intensity, changing signs as the sign of the electric field changes. The piezoelectric effect has been observed in natural quartz crystals, semicrystalline polyvinylidene, some ceramics, and human bone [12]. Either lead zirconium titanate (PZT) ceramics or polyvinyl fluorides 
(PVDF) are typically used for piezoelectric actuators. The actuators used in this research were pairs of thin PZT ceramic plates that could be easily bonded to the surface of the flexible fin. However, in the finite element model, a thermal analogy was employed to simulate the piezoelectric effect. Piezoelectric ceramics have high actuation response in terms of acceleration or displacement per volt due to the high Young's modulus inherent in the material, being able to develop strains on the order of $0.1 \%$ [13]. A high bandwidth (on the order of $100 \mathrm{kHz}$ ) and a large temperature range (-20 to 200) allows them to be used in a wide range of dynamic control applications [13]. The principle drawbacks are a high density and brittleness [12].

Polycrystalline materials composed of piezoelectric crystals are made piezoelectric by a process called poling. For ceramics, this involves heating the material to just below its Curie temperature and then subjecting the material to a strong electric field to align the electric dipoles in the ceramic with the direction of the applied field, commonly referred to as the "poling direction". The material is then cooled, locking the dipoles in their aligned orientation. Once the electric field is removed, the ceramic is left with a net dipole moment in the poling direction that is now considered to be the primary direction that can be strained when subjected to an electric field. An opposite electric field results in opposite strain. A limit is imposed on the strain that can be developed because too high an applied electric field will cause the material to repole. The primary direction is always in the same direction as one of the common axes of each crystal. The remaining two crystal axes remain oriented randomly about the aligned axis, thus making poled ceramics transversely isotropic materials with respect to the poling direction.

\subsubsection{Adaptive Structures}

In general, an adaptive structure consists of a host structure with a system of sensors and actuators directed by a controller that is capable of modifying the response of the combined system in real time in a desired fashion to environmental and operational excitations [14]. In the case of the flexible fin, it refers to the combination of piezoelectric actuators and sensors, and the control system to control vibration of fin. The sensors measure the response 
of the fin and the actuators apply forces and moments to counteract the vibration. The configuration of these sensors and actuators is critical to the ability to reduce vibration for anticipated operational conditions (excitation frequencies).

\subsubsection{Genetic Algorithms}

A genetic algorithm is an optimization technique inspired by evolution in nature, whereby a Darwinian survival-of-the-fittest approach is combined with an efficient information exchange system [15]. In nature, performance is gauged by the ability of an organism to survive; in a genetic algorithm, performance is measured by a problem specific user defined fitness function [16]. A genetic algorithm incorporates characteristics of both random and directed search techniques. A large initial population of trial solutions is randomly generated and the best solutions, subject to a fitness function, are kept while the others are discarded. The type of genetic algorithm used depends on the problem. In many genetic algorithms binary coding is used, where the individuals (trial solutions) are represented as strings consisting of 0 and 1 bits (alleles) [17]. Other genetic algorithms use continuous variables to represent individuals. A genetic algorithm is able to locate near-optimum solutions to large and difficult search problems quite rapidly. Unlike many conventional optimization techniques, many points in the search space are considered simultaneously and random choice is used to guide the search [15]. A genetic algorithm is especially applicable to surface fitting because it does not require any prior information about the function (such as its derivatives), and, given a sufficiently large initial population and number of generations for convergence, will always find the best solution. For example, increasing the initial population size reduces the chance of getting stuck at local minima. While the population is initially randomly chosen, chance is used efficiently to converge to the final solution. In all genetic algorithms, the operators of selection, reproduction, and mutation are applied sequentially on an initial random population. Some genetic algorithms incorporate other features, such as an immigration operator, or crossover before, after, or instead

of reproduction. Reproduction and crossover are quite simple operations, yet they give genetic algorithms most of their searching power. They efficiently combine and exchange 
information from the fittest individuals in a generation, ensuring that the next generation will be at least as fit, if not more fit. This gives genetic algorithms the ability to converge to a solution over many generations. Following reproduction, mutation is applied, which enhances the ability of a genetic algorithm to find a near-optimum solution by introducing new alleles into the population. This can allow the final solution to incorporate information that was not included in the initial population. Although mutation is critical to introduce new information, it should be noted that it occurs with a small probability and also that most of the mutations are detrimental. The mutations that do not improve the solution are subsequently removed in the selection process. A very small number of beneficial mutations occur, but these contribute vital information to the final solution. The fact that most mutations are detrimental means that too high a mutation rate will slow convergence by detrimentally changing the fittest individuals of the population too often; however, too low a mutation rate will also slow convergence by not introducing sufficient mutations.

\subsubsection{Combination of Finite Element Methods with Genetic Algorithms}

Optimization of the placement or grouping of piezoelectric actuators and sensors using genetic algorithms has been examined in the literature but these studies have consistently used an analytical solution in order to derive the fitness (cost) function for optimization. For example, Wang, Quek, and Ang [18] used a genetic algorithm combined with an analytical approach to optimize the positions of actuators and sensors on flexible composite plates. Kwak, Heo, and Han [19] proposed a technique for dynamic modelling a rectangular plate with arbitrarily oriented piezoelectric sensors and actuators based on the RayleighRitz method. Although a genetic algorithm was subsequently employed to determine the optimal positions of a pair of sensors and a pair of actuators simultaneously fixed on the plate, their method was also based on an analytical approach. Sheng and Kapania [20] introduced the combination of a finite element model (FEM) together with a genetic algorithm solver, although an analytic formulation was still employed. Lopes, Steffen, and Inman [21] again used an analytical approach in order to optimize the position of actuators and sensors. The most critical (and often most challenging) feature of optimization, 
particularly with a genetic algorithm, is the derivation of a fitness function. A consistent limitation of existing approaches is that they require the derivation of a closed-form fitness function for optimization, thus reducing and limiting the complexity of problems that can be solved. In contrast, the advantage of the optimization approach presented in this thesis lies in the simplicity of the fitness function and the applicability of the technique to any configuration of sensors and actuators on a structure with basically any degree of geometric complexity.

For this research, a finite element model of the fin was used to develop the fitness function from the frequency response function (FRF). A genetic algorithm was then used to optimize the configuration of a selected number of piezoelectric actuators or the position of a single actuator on the fin. The decoupling of data acquisition from the optimization process, eliminating the need for derivation of a closed-form solution, is the main advantage of the present technique in comparison to previous approaches that are reported in the existing literature. It can therefore be employed for complex geometries where analytical solutions cannot be obtained, or where derivation of such a solution would be prohibitively difficult. This method is somewhat reminiscent of that employed by Cook and Crossley [22], who optimized the position of smart actuator placement for aircraft maneuvering using a genetic algorithm. However, a major difference lies in the derivation of the fitness function; in that study PMARC (a fluid dynamics software package) was used to determine fitness of the population at each generation as the algorithm converged, instead of initially specifying a numerical description of the population fitness.

\subsection{Objectives}

There were three main objectives of the research described in this thesis: the optimization of a single pair of piezoelectric actuators on the fin using a continuous fitness function, the optimization of the configuration of the fin with multiple actuators, and verification of the simulation results by comparison with results obtained from an experimental model of the fin. To accomplish the first objective, a genetic algorithm was used for polynomial surface 
fitting of the FRF in order to obtain a continuous fitness function. To accomplish the second objective, the fitness function was derived directly from the FRF measured from the finite element model of the fin which had 48 possible pairs of actuators, completely covering the examined area without overlap. The final objective was accomplished by the comparison of an optimization where the fitness function was determined using the finite element model with an optimization where the fitness function was obtained by direct measurement of the FRFs from an experimental model of the fin with 12 pairs of actuators.

One of the main advantage of the method presented in this thesis is that it can be extended to practically any geometry or sensor and actuator configuration. Provided a structure can be modelled using finite element methods, the configuration of sensors and actuators on it can be optimized. This is especially useful for complex structures for which the derivation of a closed form fitness function is impossible or prohibitively complex. 


\section{Chapter 2}

\section{Flexible Fin Overview and Data Extraction}

The integration of actuators and sensors with the host structure often makes it prohibitively complex, if not impossible, to develop a mathematical model for a complex smart structure. It is often possible to adopt one of two alternative approaches. The structure can in some cases be reduced to an equivalent experimental apparatus on which actual actuators and sensors can be applied. Modal and other methods of vibration analysis can be applied to this model, and the results can be scaled to correspond to the actual structure. A control system can then be developed and tested on the experimental apparatus. A much simpler and usually reasonably accurate approach is to use a finite element model of the structure to extract vibrational information and develop a suitable control system. In this thesis, both the experimental and finite element approaches are adopted to investigate the problem of optimizing the configuration of piezoelectric actuators on a scaled model of a flexible aircraft fin.

\subsection{Finite Element Methods Overview}

Finite element methods are often used to model and predict the dynamic response of a structure with integrated sensors and actuators. An excellent early example of this is the study by Rahmoune et. al [23], where the electromechanical coupling effect of piezoelectric materials was used to establish a finite element model of a flexible plate with bonded piezoelectric sensors and actuators. At the National Research Council of Canada, a finite 
element model of the smart fin discussed in this thesis was used as one of the bases for the development of the active control for the fin [4].

\subsection{Fin Configurations}

The same flexible fin model was used for all parts of this research, although the actuator configurations were modified to accomplish the objectives described in Chapter 1 . The same basic finite element model of the fin, that represented the experimental apparatus, was used for all optimizations. The experimental configuration will therefore be described first, although the results it yielded are discussed in Chapter 5 while results from the finite element model optimizations are discussed in Chapters 3 and 4 . The general shapes of the first three modes are shown in Figure 2.1.

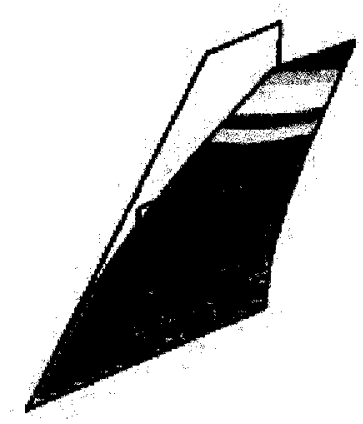

(a) First Mode

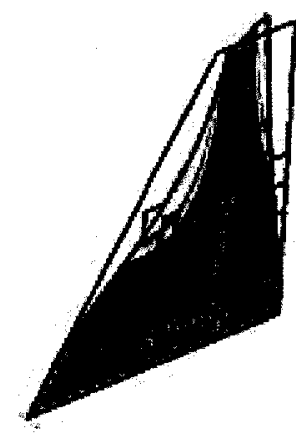

(b) Second Mode

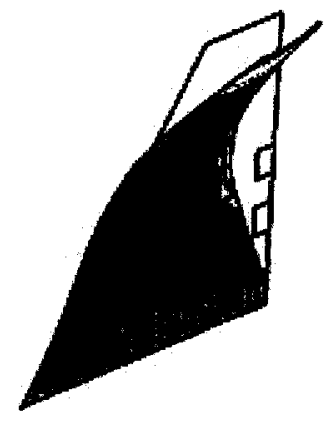

(c) Third Mode

Figure 2.1: The Fin Mode Shapes

\subsubsection{Experimental Set-up vs. Finite Element Model}

The experimental model of the flexible fin that was developed at the ASDSMP laboratory of the National Research Council of Canada was used for the purposes of this study. It consisted of a flexible aluminum fin of $1 \mathrm{~mm}$ thickness cantilevered at the fin root between a pair of solid aluminum blocks. Twelve lead zirconium titanate (PZT) thin ceramic plate actuators (BM500 from SensorTech Systems, Inc.) were bonded to each side of the fin [4]. 
A schematic of the model is shown in Figure 2.2. The material properties of the components and the natural frequencies and damping ratios of the integrated structure are discussed in Chapter 5. A finite element model of the integrated fin with accelerometers and actuators positioned in the same locations as in the experimental apparatus was used for comparison of experimental and simulation results. This equivalent finite element model is presented in Figure 2.3.

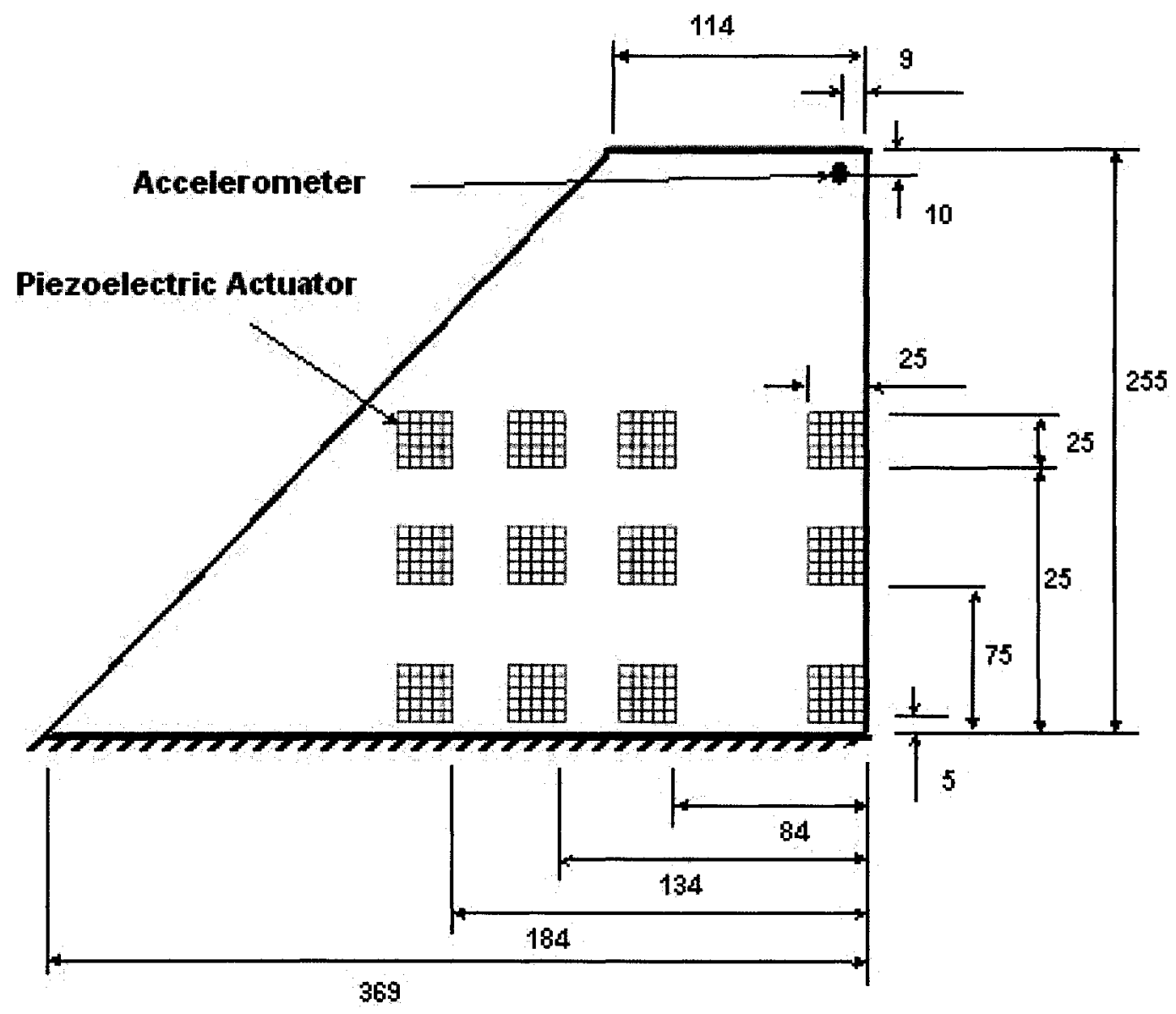

Figure 2.2: Geometry of the Smart Fin in Experimental Configuration

\subsubsection{Accelerometers}

For both the experimental and finite element models, five accelerometers were used to measure the dynamic response of the integrated smart structure. These were positioned so 


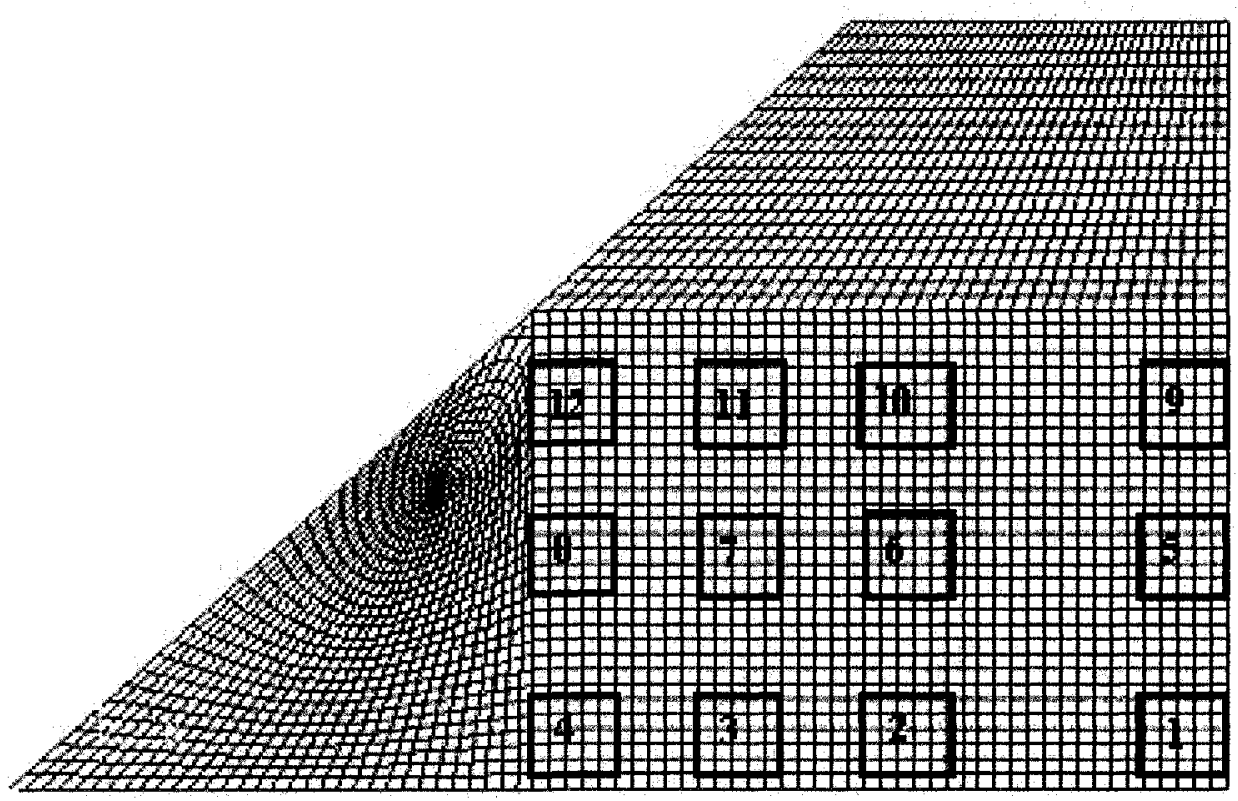

Figure 2.3: Finite Element Model of the Smart Fin in Experimental Configuration [4]

that they could measure relatively high responses from the first three dynamic modes of the smart fin. The positions of these accelerometers are shown in Figure 2.4, and are listed in Table 2.1. All coordinates refer to the centroid of each accelerometer.

Table 2.1: $\mathrm{X}$ and $\mathrm{Y}$ Accelerometer Positions on the Smart Fin along with Corresponding Finite Element Node

\begin{tabular}{cccc}
\hline Accelerometer & $\mathrm{X}(\mathrm{mm})$ & $\mathrm{Y}(\mathrm{mm})$ & Finite Element Node Number \\
\hline \hline 1 & 8.8 & 245.4 & 10010 \\
2 & 181.7 & 178.2 & 7688 \\
3 & 114.8 & 245.4 & 7778 \\
4 & 10.0 & 168.2 & 9996 \\
5 & 8.8 & 210.2 & 10040 \\
\hline
\end{tabular}

The accelerometer positions were chosen in order to be well suited to measure the response of all modes, specifically, accelerometer 1 was positioned at the fin tip trailing edge, as far as possible from the node lines associated with the lower fundamental modes. As such, it was well positioned to record a substantial response when the fin was excited at all 


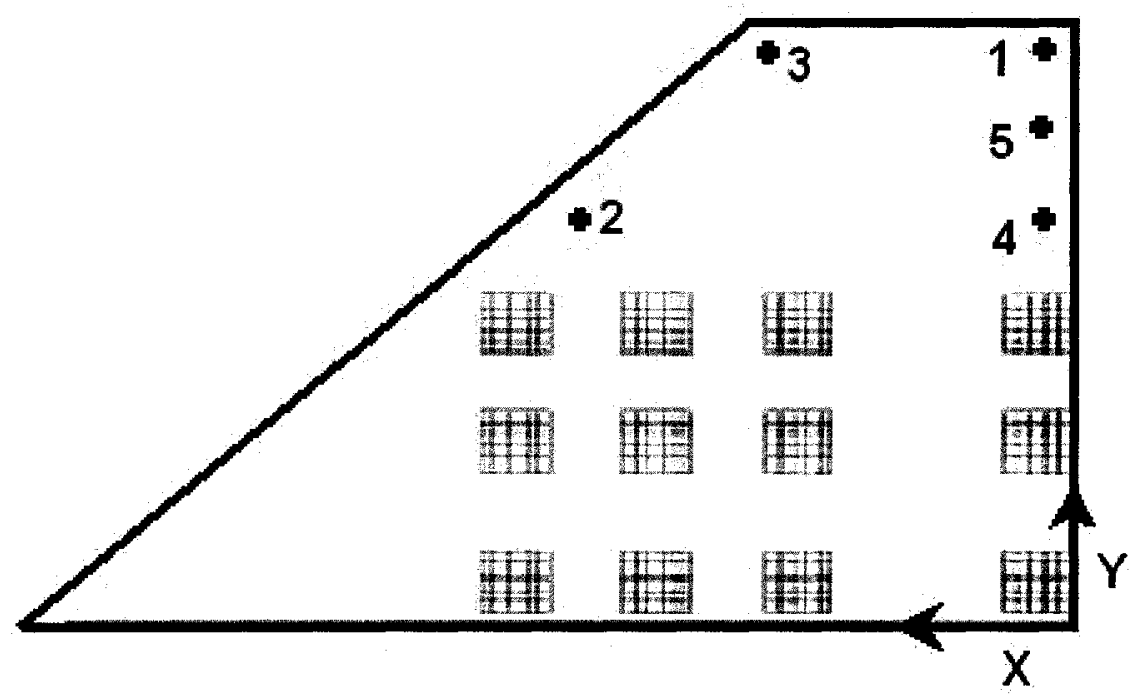

Figure 2.4: Accelerometer Positions on the Smart Fin

three of the first fundamental frequencies, and the response measured by it was used for the subsequent optimization of actuator position for each mode. The other accelerometers were primarily used for reference and verification of data, and no optimization was performed using the data they recorded. Accelerometer 2 was positioned midway from root to tip at the leading edge, and as such, it was well positioned to record specifically the response of the fin when excited at the second fundamental frequency. Similarly, accelerometers 3,4 , and 5 were well positioned to record the response at the first and third, second, and second and fourth fundamental frequencies, respectively. In general, a complex frequency response function contains both the magnitude and phase of a steady state response [24]. For the purposes of this work, since the magnitude was the primary concern, the FRF magnitude was taken as the acceleration recorded at accelerometer 1 divided by the input voltage in order to normalized the response.

\subsubsection{Finite Element Model for Actuator Configuration Optimization}

For both the single and multiple actuator configuration optimization, the same basic finite element model with 48 possible actuator locations (6 rows of 8 actuators) was used, Figure 
2.5. In both cases, and for each vibration mode, the resulting FRF at accelerometer 1 was measured once for each of the 48 possible actuator positions. However, in the case of the single piezoelectric actuator optimization problem, a single pair of actuators was bonded sequentially at each of the 48 different positions, whereas in the case of the actuator configuration optimization problem, each actuator was sequentially activated from the 48 bonded actuators that entirely covered the pre-determined region for all runs.

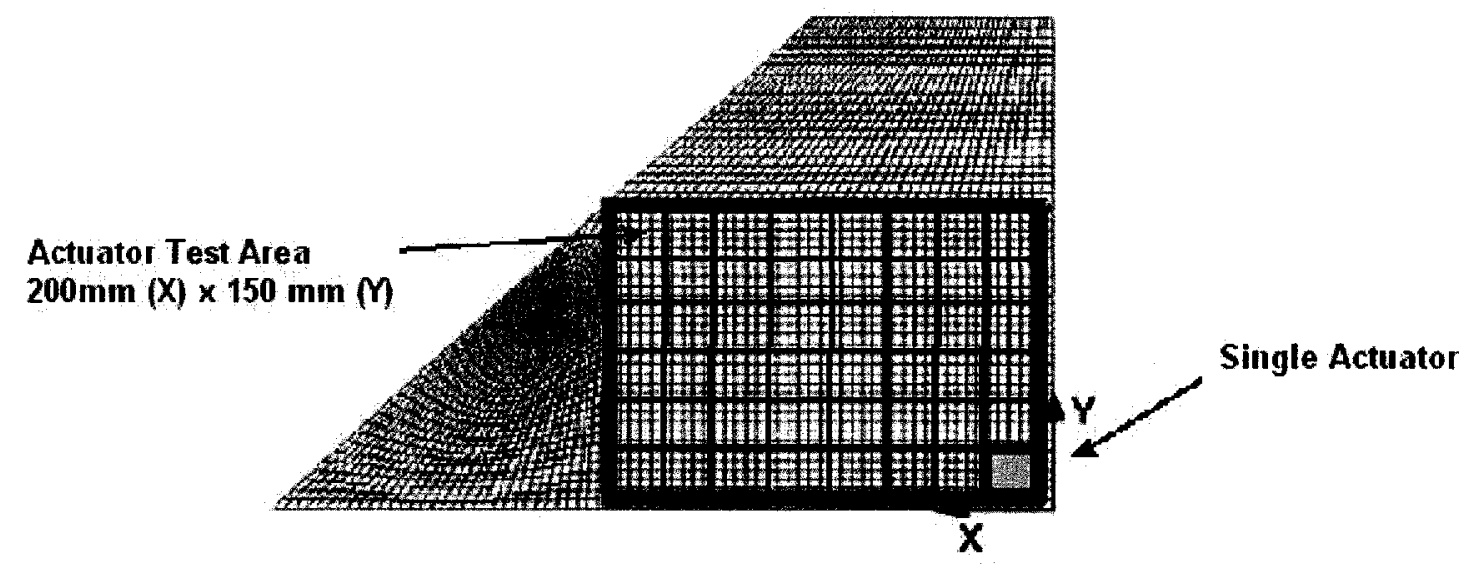

Figure 2.5: The 48 Actuator Positions from the Finite Element Model of the Smart Fin

\subsection{Finite Element Method Data Acquisition}

A basic FEM model of the integrated smart fin that was available at the ASDSMP laboratory of the National Research Council was used in this study. This model was developed using the MSC Patran software package and MSC Nastran was employed as the finite element model frequency response solver. A thermal load analogy was used to simulate the piezoelectric effect because it is not represented in Patran as is the case with most finite element software packages. The following specific assumptions are incorporated into the FEM model:

- a perfect bond between the host structure and the actuators,

- negligible change of mass and stiffness due to the bonding agent, and 
- incorporation into the model of a general structural damping ratio of $3.4 \%$ based on experimental results. This damping ratio was determined by trial and error by equalizing the first mode response from the experimental and FEM models. This assumption led to a requirement to scale the results for the second and third modes in Chapter 5.

\subsubsection{FEM Elements and Configuration}

Shell elements and solid elements were employed in Patran for the aluminum plate and actuators, respectively. The actuator solid elements were connected at their base to nodes on the aluminum plate shell elements. Each actuator was modelled as 25 solid $5 \mathrm{~mm} \times$ $5 \mathrm{~mm} \times 1 \mathrm{~mm}$ HEX20 (20 nodes) elements in PATRAN; an example is shown in Figure 2.6. The aluminum fin was modelled within the actuator test area as $5 \mathrm{~mm} \times 5 \mathrm{~mm}$ square QUAD8 shell elements of $1 \mathrm{~mm}$ thickness, each having nodes at their corners as well as mid-side nodes, for a total of 8 nodes per shell element. Outside the actuator test area, the elements varied in size and shape, as shown in Figue 2.5. It was critical that the face of each solid element was consistent in size and shape with the shell elements inside the actuator test area. However, the size and shape of the elements outside this area was unimportant and the resulting variation in element geometry was necessary to create the fin shape.

\subsubsection{Thermal Analogy}

Since only a few commercial software packages such as ANSYS are capable of piezoelectric modelling, a thermal analogy was employed in Nastran to model the piezoelectric actuators. For low frequency ranges and low applied voltages, the induced strain is given by:

$$
\varepsilon=\frac{d_{31}}{t} \Delta V
$$

where $d_{31}$ is the piezoelectric charge constant for the primary poled direction. $\Delta \mathrm{V}$ and $\mathrm{t}$ represent the applied voltage, and thickness of the piezoelectric actuators, respectively. Although there are strains in the perpendicular direction, the focus in this work is on strain 

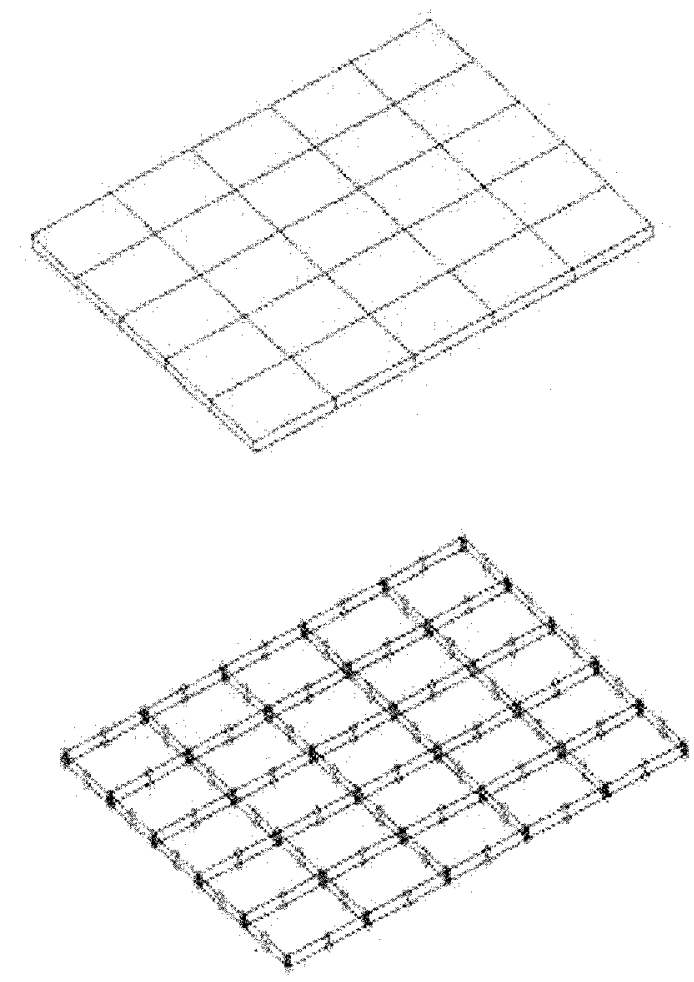

Figure 2.6: Actuator Modelling using 25 HEX20 Solid Elements [4]

in the primary poling direction. Thus, the induced strain is linearly proportional to the applied voltage and, in effect, it is analogous to thermal strain. Substituting for the thermal coefficient, $\alpha$, the thermal analogy can be used to simulate the piezoelectric effect, i.e.,

$$
\varepsilon=\alpha \times \Delta T
$$

where

$$
\alpha=\frac{d_{31}}{t}
$$

and $\Delta \mathrm{T}=\Delta \mathrm{V}$. 


\subsubsection{Patran and Frequency Response Simulation}

The first step was to set up the finite element model with only the aluminum fin modelled by shell elements. The geometry of the shell elements within the actuator test area was strictly constrained to $5 \mathrm{~mm} \times 5 \mathrm{~mm}$ in order to later accommodate the actuator solid elements. The cantilevered fixed root constraint was then applied to the fin. Next, a thermal constraint setting the temperature on the surface of the plate to zero was applied. This was necessary to prevent heat transfer into the plate that would arise from the thermal analogy used to represent the piezoelectric effect. If this constraint was not applied, the aluminum fin itself would contribute to this piezoelectric effect. This fin with the cantilevered constraint was used for both the single actuator optimization problem and the multiple actuator configuration problem.

For the single actuator optimization problem, a single pair of $25 \mathrm{~mm}$ by $25 \mathrm{~mm}$ actuator of solid elements was created at the first position (see Figure 2.5) and exposed at its outer face to a unit magnitude thermal load. A modal analysis was run using MSC Nastran with thermal cyclic loading in order to determine the first three fundamental frequencies of the finite element model of the integrated structure. The first three mode shapes are shown in Figure 2.1. The first three fundamental frequencies of the structure with a single actuator at the first position were determined to be $17.0 \mathrm{~Hz}, 49.1 \mathrm{~Hz}$, and $80.4 \mathrm{~Hz}$. Since only a single piezoelectric actuator was used, these fundamental frequencies remained relatively constant wherever the actuator was positioned on the fin. A "vibration analysis" run was then set up for simulation using MSC Nastran. A structural damping ratio of $3.4 \%$ was selected and the thermal load on the actuator outer surface was selected as the varying load. This was selected by equating the peak FRF magnitudes from the experimental model with those from the finite element model for the first mode. This will later lead to some scaling requirements in Chapter 5 for the second and third modes. The frequency range for simulation was selected as $0.1 \mathrm{~Hz}$ increments of $+/-2 \mathrm{~Hz}$ around the first three fundamental frequencies $(15-19 \mathrm{~Hz}, 47.1-51.1 \mathrm{~Hz}$, and $78.4-82.4 \mathrm{~Hz})$. A bulk run, with OP2 (output 2) output file format was selected, and the run was ready for analysis in MSC 
Nastran. This was then repeated for 47 other runs at each of the actuator positions specified in Figure 2.5.

The Patran section of the multiple actuator configuration optimization problem was a similar process. The only difference in the model was that instead of a new actuator being created in each of the 48 possible actuator positions, at the start the 48 actuators were created with no thermal loads, and a single thermal load was moved sequentially to each of the 48 actuators. The simulated frequency range was also adjusted to correspond with a $2 \mathrm{~Hz}$ range around the new first three fundamental frequencies of the integrated structure. Although the finite element model for this optimization had 47 passive actuators for each FRF measurement, the compromise between stiffness and mass contributed by the passive actuators, in fact resulted in less than $10 \%$ difference in fundamental frequency for all three modes as compared with a single actuator on the fin $(17.5 \mathrm{~Hz}, 47 \mathrm{~Hz}$, and $73 \mathrm{~Hz})$.

\subsubsection{Nastran and Post Processing Results}

MSC Nastran was used as a solver for all simulations of the finite element model. A Nastran simulation took just under one hour per run. Usually there were no problems with the simulation and a message appeared confirming the end of simulation. Occasionally, there was a problem with the finite element model and the output files could be consulted to determine the nature of the problem so that action could then be taken to eliminate it. Once the simulation was successfully completed, the Nastran output was loaded in Patran for post processing. This results file included vibration response information for every node on the fin at each of the requested frequencies. A report file was requested that included the resultant peak acceleration in the z-direction (out of the plane of the fin) at each of the nodes representing the accelerometers listed in Table 2.1. This report was sorted by node and hence by accelerometer for each of the frequency cases, and listed the accleration magnitude. The Patran report file format, .rpt, could be read as a text file, or loaded in Microsoft Excel to preserve the tabular structure. 


\subsubsection{Excel Files and Selection of Peak FRF}

The Patran report files were opened in Microsoft Excel and the columns were adjusted on initial opening in Excel to conform to the tabular structure used in the report files. This created Excel files containing all the acceleration response data for the nodes corresponding to the accelerometers listed in Table 2.1 at the frequency ranges of $+/-2 \mathrm{~Hz}$ from the first three fundamental frequencies in $0.1 \mathrm{~Hz}$ increments. These Excel files were convenient to work with, and since the thermal load applied to the actuators was of unitary magnitude, the resultant acceleration was in fact exactly equal to the frequency response function (FRF). Since the objective was to minimize the vibration amplitude for the first three modes, the FRF magnitude recorded by accelerometer 1 was used as a basis for optimization. The peak FRF for each of the first three fundamental frequencies was selected for each of the 48 actuator positions. This provided a direct measure of peak FRF over the surface of the actuator test area of the fin for each mode. An example of part of a typical Excel file is shown in Figure 2.7, and a typical peak FRF recorded by accelerometer 1 with the actuator positioned at all 48 actuator location is shown in Figure 2.8. The frequency cases are specified in Patran and are spaced in requested frequency increments. In this case, the frequencies are spaced at intervals of $0.1 \mathrm{~Hz}$ and occupy a range of $+/-2 \mathrm{~Hz}$ around each of the first three fundamental frequencies of oscillation. 


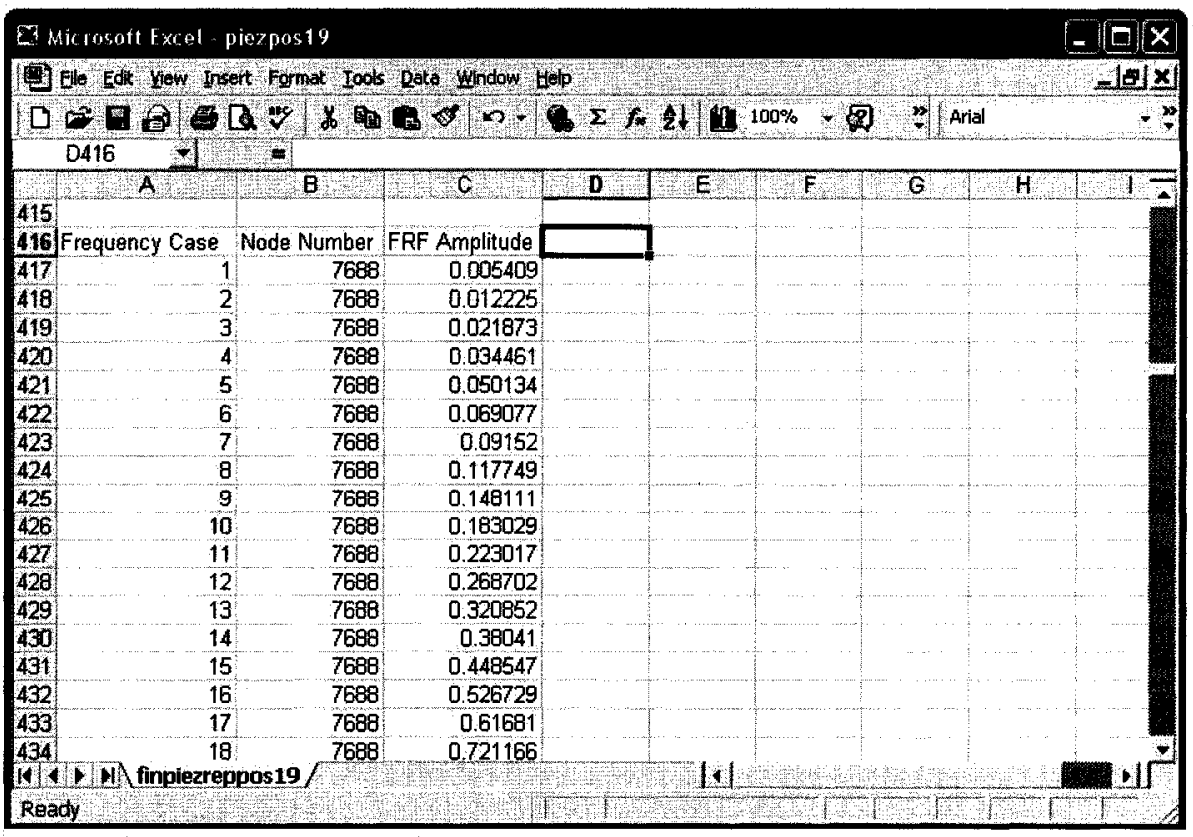

Figure 2.7: Example of Excel Results File for Extraction of Peak FRFs

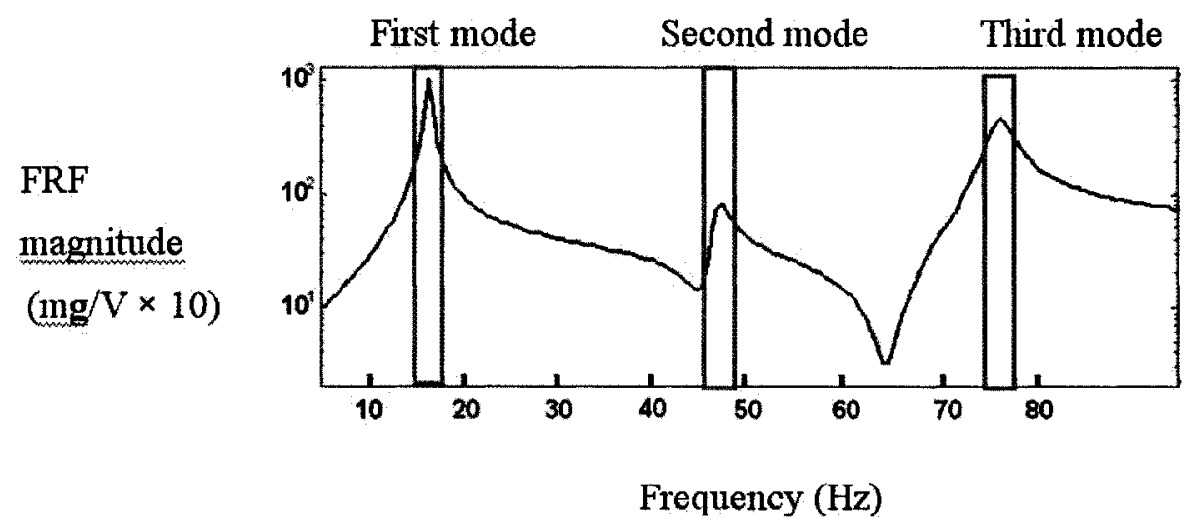

Figure 2.8: Peak FRF at Accelerometer 1 with the Actuator at each of the 48 Possible Positions 


\section{Chapter 3}

\section{Optimization of the Position of a Single Pair of}

\section{Actuators}

This chapter describes the optimization of the position of a single pair of piezoelectric actuators within the actuator test area of the fin to achieve a maximization of actuation authority for either individual control of the first three modes or for control of a combination of the modes simultaneously. The overall approach is described and then the procedure and results are discussed in detail.

\subsection{Approach: Polynomial Surface Fitting to Obtain a Con- tinuous Fitness Function}

In order to optimize the position of a single pair of piezoelectric actuators continuously within the actuator test area, a continuous fitness function describing the actuation authority of a pair of actuators anywhere within the test area was required. This fitness function was developed using a genetic algorithm designed to generate the best three-dimensional polynomial surface fit of the piezoelectric actuator FRF peak values within the test area, and to a limited degree by extrapolation, outside of the test area as well. Using this fitness function, a second genetic algorithm was developed to determine the optimum position for the actuator pair in order to maximize actuation authority for each mode individually, or for a combination of the modes, with modal emphasis achieved through the use of weighting 
factors on the combined modal fitness function.

\subsubsection{Suitability of Three-Dimensional Polynomial Surfaces}

Three dimensional polynomial surfaces were used to represent the magnitude of the FRF generated by a single pair of actuators anywhere within the actuator test area on the flexible fin. The $\mathrm{x}$ and $\mathrm{y}$ coordinates of the polynomial surface represent the position of a pair of actuators, while the magnitude of the polynomial represents the corresponding actuation authority recorded at the fixed accelerometer in terms of peak acceleration per volt (FRF), or peak displacement per volt, where the voltage is input into each actuator. It is analogous to a two-dimensional polynomial curve fitting of data, except it is much more complex because the data correspond to a point on a surface rather than on a line. As with curve fitting, polynomial surfaces are excellent to represent relatively steady patterns with few maxima and minima. Similar to a polynomial curve, a polynomial surface takes the form:

$$
\begin{gathered}
z=a_{1}+a_{2} x+a_{3} y+a_{4} x^{2}+a_{5} x y+a_{6} y^{2}+\ldots, \\
\text { Fitness }=\frac{1}{\text { Error }} .
\end{gathered}
$$

where $\mathrm{z}$ is the magnitude of the FRF peak value achieved at the accelerometer position for a corresponding $\mathrm{x}$ and $\mathrm{y}$ actuator pair position. The polynomial coefficients are represented by $a_{1}, a_{2},, a_{n}$, where $\mathrm{n}$, the number of coefficients, depends on the order of the polynomial sought. The values of $n$ for polynomials up to ninth order are shown in Table 3.1.

Table 3.1: Number of Coefficients for a Polynomial Surface by Polynomial Order

\begin{tabular}{lcccccccccc}
\hline \hline Polynomial Order (p) & 0 & 1 & 2 & 3 & 4 & 5 & 6 & 7 & 8 & 9 \\
No. of Coefficients (n) & 1 & 3 & 6 & 10 & 15 & 21 & 28 & 36 & 45 & 55 \\
\hline
\end{tabular}

An exact polynomial fit for $\mathrm{n}$ data points is achieved with an $\mathrm{n}-1$ order polynomial, 
but it is usually desirable to use a simpler polynomial if a sufficiently low error can be achieved, since the increase in the number of coefficients means that it is much more computationally demanding to use high order polynomials [25]. For example, although a 47th order polynomial would have resulted in an exact representation of the FRF data at each of the 48 possible actuator positions on the flexible fin, it was often possible to use a low order polynomial (such as 3rd order) to adequately represent the data over the entire area. Conversely, it may not be possible to obtain a single polynomial surface that fits the data accurately over the entire area without using a prohibitively high order (such as 10th order or higher). Also, polynomials are not good choices to represent all data sets. The set should not be overly erratic and there should not be an abundance of minima and maxima. In some cases polynomials are not suitable to represent the data set as a whole, but they are suitable to represent the data set within subregions of the area. This also occurs with two dimensional polynomial curve fitting and the solution is to subdivide the region and use separate curves known as splines for each subregion. This strategy was adopted here for surface fitting in the case of the second mode. Instead of fitting a single polynomial surface to the entire actuator test area, separate polynomial surfaces were used to represent the peak FRF magnitudes in each of four neighbouring subregions of the test area. When the neighbouring subareas are combined, this strategy offers a very good representation of the peak FRF magnitude over the entire actuator test area.

\subsubsection{Fitness Function for Polynomial Surface Fitting}

Two fitness functions were used in optimizing the position of the single pair of piezoelectric actuators on the flexible fin: one for each of two genetic algorithms. The first genetic algorithm was designed to determine the optimum polynomial surface fit of the peak FRF magnitudes in order to develop the fitness function to use in the second genetic algorithm for actual positional optimization. The development of the fitness function that is used in the genetic algorithm for the positional optimization is discussed later in this chapter.

For polynomial surface fitting, the obvious choice as a fitness function is some kind of inverse representation of the difference between the trial polynomial FRF peak magnitude 
and the actual FRF peak magnitude, because this difference (called error) is to be minimized in order to match the trial polynomial to the FRF data. Therefore, an appropriate fitness function for polynomial surface fitting is:

$$
\text { Fitness }=\frac{1}{\text { Error }}
$$

The simplest definition of error for this type of fitness function would be the sum of the absolute values of the errors, or first order sum error:

$$
\text { First Order Sum Error }=\left|z_{1}-d_{1}\right|+\left|z_{2}-d_{2}\right|+\ldots+\left|z_{48}-d_{48}\right|
$$

where $z_{i}$ is the FRF peak magnitude value given by trial polynomial surface, and $d_{i}$ is actual FRF peak magnitude at the acelerometer for the ith position of the actuator. The first order sum error can be used for polynomial surface fitting, but a much faster convergence rate can be achieved by increasing the emphasis of outliers in the population. By using a least-squares (LS) evaluation of peak FRF magnitude error for the fitness function, the contribution of coefficients with a significant error is highlighted, and, in effect, the genetic algorithm concentrates the majority of its searching ability on improving these coefficients. A LS curve fitting approach was employed by Karr to emphasize outlying data points in order to maximize convergence rate [16]. Using a LS approach, the fitness function was defined as the inverse of the square root of the sum of the squares of the residuals (SRSS):

$$
\begin{gathered}
\text { Fitness }=\frac{1}{\text { SRSS Error }} \text {, and } \\
\text { SRSS Error }=\sqrt{\left(z_{1}-d_{1}\right)^{2}+\left(z_{2}-d_{2}\right)^{2}+\ldots+\left(z_{48}-d_{48}\right)^{2}} .
\end{gathered}
$$

Combining a LS fitting technique with a genetic algorithm has been suggested by Karr, Stanley, and Scheiner [26], however they restricted their research to two dimensional curve fitting. Their research also suggested a least median square (LMS) based fitness function, where the median of the squared residuals is used as an error measurement. In the surface fitting algorithm, a LS based fitness function yielded better convergence rates. It is also 
possible to use higher order errors for the fitness function. For example, fourth and sixth order errors were also considered. However, it was determined that a fitness function using the SRSS error provided the best convergence rates and this fitness function was used in the genetic algorithm for polynomial surface fitting.

\subsubsection{Convergence of Solution}

An appropriate convergence criteria is essential in order to evaluate the ability of the genetic algorithm to obtain an accurate polynomial surface fit. The simplest method to evaluate the surface fitting algorithm is to examine an error vs. generation plot for the best coefficient set in the population at each generation. The error for this plot can be defined in several ways. It can be in a first order sum or least squares form as in Equations 3.4 or 3.6. Alternatively, the maximum, minimum, or mean errors can be examined. These are defined as:

$$
\begin{gathered}
\text { Maximum Error }=\max \left|z_{i}-d_{i}\right|, \\
\text { Minimum Error }=\min \left|z_{i}-d_{i}\right| \text {, and } \\
\text { Mean Error }=\frac{\left|z_{1}-d_{1}\right|+\left|z_{2}-d_{2}\right|+\ldots+\left|z_{48}-d_{48}\right|}{48} .
\end{gathered}
$$

A major drawback of all the error measurements highlighted so far is that they are defined in absolute terms, and therefore require some information about the data set in order to asses the goodness of fit. The error measures can be improved by normalizing them as a percentage of the mean FRF peak value. This offers a better indication of the accuracy of the surface fit since the error is scaled to the data magnitude:

$$
\text { Normalized Error }=\frac{\text { Error }}{\left|d_{1}\right|+\left|d_{2}\right|+\ldots+\left|d_{48}\right|}
$$

The convergence rate and final error can be determined from error vs. generation plots as shown in Figure 3.1. 


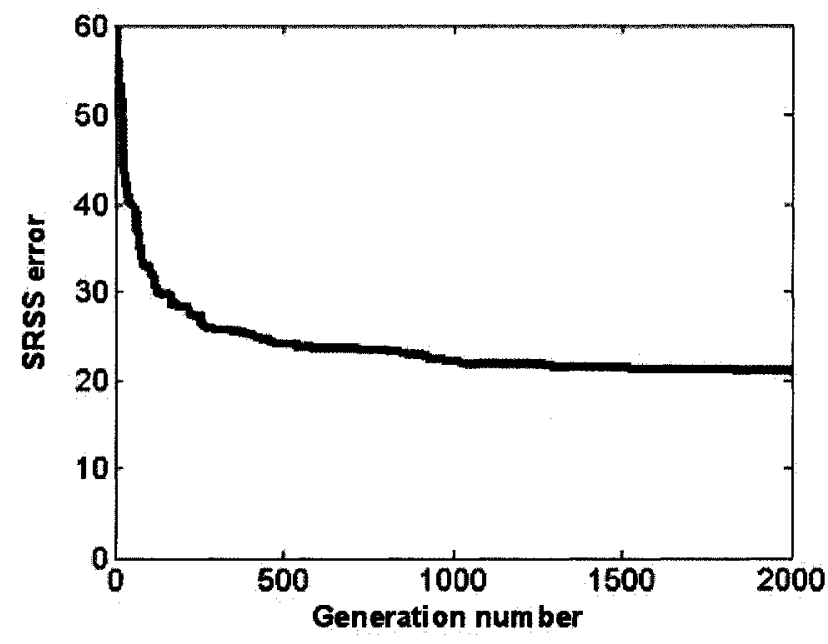

(a) SRSS Error vs. Generation

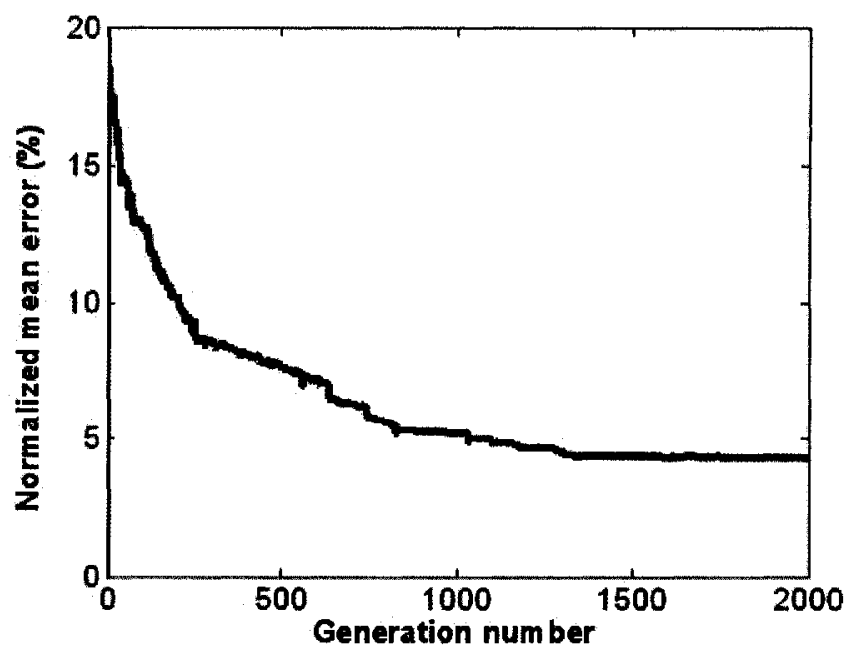

(b) Normalized Mean Error vs. Generation

Figure 3.1: Typical SRSS Error (left) and Normalized Mean Error (right) vs. Generation Plots

Another parameter that can be used to evaluate error is R-square [27]. R-square is typically used to evaluate goodness of fit for 2-D curve fitting, but it can also be expanded in order to be applied to 3 -D surface fitting. It takes a value between 0 and 1 with a value closer to 1 representing a better fit. R-square is defined as the sum of squares of regression (SSR) divided by the sum of squares about the mean (SST):

$$
R-\text { square }=\frac{S S R}{S S T}
$$

The sum of squares of regression is defined as

$$
S S R=\sum_{i=1}^{48}\left(z_{i}-\bar{d}\right)^{2},
$$

and the sum of squares about the mean is defined as

$$
S S T=\sum_{i=1}^{48}\left(d_{i}-\bar{d}\right)^{2} .
$$

$\mathrm{R}$-square is a simple yet effective non-dimensional parameter to asses the accuracy of the surface fit. An inability of the genetic algorithm to obtain an R-square value close to 
1 (taking for example 0.9 or higher as a reasonable value), indicates that the data set does not lend itself well to polynomial surface fitting. The improvement of surface fit that results from using subdivided regions is evident from $\mathrm{R}$-square and the normalized error, as will be demonstrated later.

\subsection{Genetic Algorithm for Surface Fitting}

A genetic algorithm incorporates characteristics of both random and directed search techniques. A large initial population of trial solutions is randomly generated and the best solutions, subject to a fitness function, are kept while the others are discarded. In many genetic algorithms binary coding is used, where the individuals (trial solutions) are represented as strings consisting of 0 and 1 bits (alleles). Alternatively, in continuous problems, the individuals are often composed of real numbered alleles. The surface fitting and single actuator position optimization genetic algorithm use real numbered alleles, while the multiple actuator configuration optimization algorithm described in Chapter 4 uses integer alleles.

While the parameters in a genetic algorithm are initially randomly chosen, they use chance efficiently to converge to the final solution. Like most genetic algorithms, the operators of selection, reproduction, crossover and mutation are applied sequentially on an initial random population. However, in the polynomial surface fitting genetic algorithm, an immigration process was also applied, in which completely new individuals were introduced after an initial selection process. Selection was then re-applied to determine if any of the immigrated individuals were fitter than any of the individuals in the old population. This allowed new information to be considered at any generation of the process, and reduced the number of generations to convergence as well as providing insurance against missing possible solutions. A new generation was then created by randomly combining the coefficients of the selected individuals to create new coefficient sets. Crossover also affords a means for strings to mix and match their desirable coefficients through a random process.

Reproduction and crossover are quite simple operations, yet they give genetic algorithms 
most of their searching power. They efficiently combine and exchange information from the fittest individuals in a generation, ensuring that the next generation will be at least as fit, if not more fit. This gives genetic algorithms the ability to converge to a solution over many generations. Following crossover, mutation is applied, which has a small chance of randomly changing each of the coefficients. Mutation enhances the ability of a genetic algorithm to find a near-optimum solution by introducing new alleles into the population. This can allow the final solution to incorporate information that was not included in the initial population.

Convergence of the solution to a final value was achieved by repeatedly performing selection, reproduction, crossover, and mutation until the fitness was sufficiently high or a specified number of generations had elapsed. The effects of population pool size, mutation rate, mutation severity, and immigration are examined here to determine the performance of the algorithm for polynomial optimization. Selection of suitable values for these parameters was critical in reducing error. For example, too high a mutation rate caused excessive divergence of the solution, while too low a rate resulted in a very long convergence time. Selection of a suitable polynomial order was also essential.

In the polynomial surface fitting algorithm, each individual was composed of a single chromosome of a single gene, and these individuals were represented with strings of real numbered alleles since the problem was in the continuous domain. In reference to Equation 3.1 , each individual was composed of a string encoding $a_{1}, a_{2}, \ldots, a_{n}$, where $\mathrm{n}$, the number of coefficients (alleles), depended on the order of the polynomial (p) sought as shown in Table 3.1. The genetic algorithm developed in this work is capable of fitting up to a 9 th order polynomial of the form of Equation 3.1.

This increase in the number of coefficients means that it is much more computationally demanding to fit higher order polynomials. The algorithm is sufficiently robust to fit most sets of any number of data points in the $x-y$ plane to a polynomial of up to 9 th order. The ability of the algorithm to obtain a suitable polynomial surface was examined at different polynomial orders. Third and eighth order polynomials were used for the first and third vibration modes. For the second mode, four neighbouring eighth order polynomials were employed to reduce the error that was associated with a single polynomial surface covering 
the entire actuator test area. Computational efficiency was not critical since the surface fitting needed to be performed only once for each modal frequency. The total run time for the most computationally demanding case encountered in this thesis was approximately 30 minutes for a 9th order polynomial surface using Matlab.

\subsubsection{Genetic Algorithm Steps}

The polynomial surface fitting algorithm incorporated the following steps:

1. The "range" parameters are derived (described below). This is used in the generation of the population pool and in mutation.

2. A large initial random pool of individuals (sets of coefficients which describe polynomial surfaces) is generated. The initial population for the genetic algorithm is obtained from this pool in step 4.

3. The trial polynomial function value at each actuator position is calculated and compared to the FRF peak value to determine the fitness (Equation 3.5).

4. The ten coefficient sets with the lowest error are selected as the first generation.

5. Reproduction and crossover are applied.

6. Each allele in each coefficient set is subjected to a small chance of mutation.

7. The error is calculated and the ten best coefficient sets are selected.

8. Immigration is applied to replace the unselected sets.

9. The error is re-evaluated and ten new individuals are selected as the next generation.

10. Steps 5 to 9 are repeated until convergence is achieved. 


\section{Derivation of the Range Parameters}

A set of values referred to in this thesis as "range" parameters were developed specifically for the surface fitting algorithm in this research. These parameters were necessary both to specify limits on the initial population pool and mutation. Without limits, both the synthesis of the population pool and the mutation operation would be completely unbounded. If unbounded, the coefficients (alleles) composing the initial population pool could vary from negative infinity to infinity, which would lead to an extremely unfit first generation and result in poor algorithm performance. Similarly, if the mutation operation was unbound, the chance of a successful mutation would be negligible. In fact, defining boundaries for mutation is not unlike what actually occurs in nature, since DNA uses a set of only four base pairs. The requirement for bounds on the alleles in the initial population pool stems from the definition of the alleles as real numbers. The use of real numbers allows the alleles to define a spectrum of characteristics, and in effect, the alleles in a real numbered system are equivalent to a gene in a binary system since they define a characteristic solely based on their value. An unbound mutation would be equivalent to a simultaneous mutation in all genes, which would be exceedingly improbable in nature or in a binary algorithm.

Since the individuals in the population were composed of $n$ coefficients as in Equation 3.1, a different range parameter is necessary for each power $(\mathrm{p})$ of coefficient. The zeroth order value of range, $r_{0}$, is defined as the absolute value of the maximum FRF peak value magnitude in the data set. The first order value of range, $r_{1}$, is defined as the absolute value of the maximum slope in $\mathrm{x}$ or $\mathrm{y}$ directions between FRF peak values in a data set. The second order value of range, $\mathrm{r}_{2}$, is defined as the maximum change in slope within the data set. The values of the range parameter are shown in Table 3.2, and the derivation of the zeroth, first, and second order values of range is shown in Figures 3.2 to 3.4. Although the higher order values of range are nominally determined from the higher order derivatives, the algorithm actually uses a near-zero integer value in order to specify bounds. The limits used for the range parameters beyond the third are not critical as long as they are small in magnitude because the actual values are usually small, though in general $r_{3} \geq r_{4} \geq r_{5} \geq r_{6} \geq r_{7} \geq r_{8} \geq r_{9}$. 
While the range parameter limits the initial coefficient values to within a specified range, these coefficients are permitted to diverge from this during the mutation or immigration processes.

Table 3.2: Values of the Range Parameters for Each Polynomial Order

\begin{tabular}{cc}
\hline \hline Polynomial Order & Corresponding Range Parameter Value \\
0 th & FRF peak value magnitude \\
1 st & Maximum slope \\
2nd & Maximum change in slope \\
3 rd & 3 \\
4 th & 2 \\
5 th & 1 \\
6 th & 1 \\
7 th & 1 \\
8 th & 1 \\
9 th & 1 \\
\hline
\end{tabular}

\section{Initial Population Pool}

The surface fitting genetic algorithm selects the first generation of individuals from a large initial population pool. This has the benefit of vastly increasing the fitness of the first generation, which dramatically improves subsequent convergence. This initial population pool is randomly generated, but is subject to the range parameters for each order of coefficient. In fact, since the initial population pool is subject to coefficient boundaries, the surface fitting algorithm is actually a random search followed by a process of natural selection in order to improve results. The combination of the random search and the natural selection process achieves a far superior surface fit to that which could be produced by either individually. The size of the initial population pool is a critical factor in convergence, and this is discussed below.

The initial population pool is randomly generated using the range parameters. Each individual is generated for an n-order polynomial of the form in Equation 3.1 one coefficient 


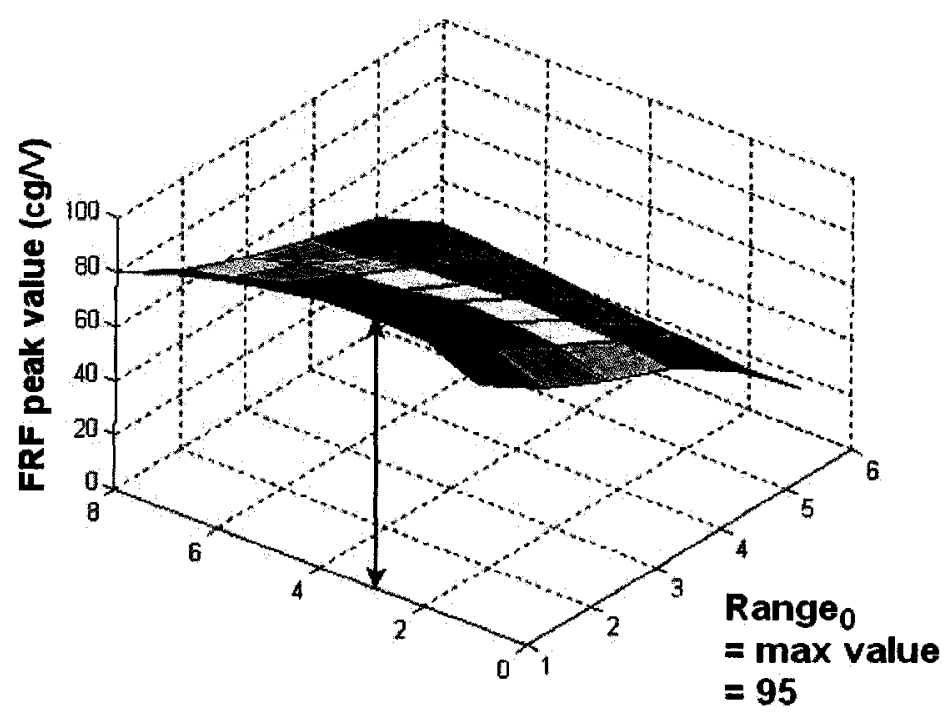

Figure 3.2: Calculation of Range Parameter $r_{0}$

at a time according to Equation 3.14, where $\alpha$ is a random number between -1 and 1, and $p$ is the polynomial order. The number of coefficients depends on the polynomial order as shown in Table 3.1.

$$
a_{1}=\alpha_{1} \text { range }_{0} ; a_{2}=\alpha_{2} \text { range }_{1} ; \ldots ; a_{5}=\alpha_{5} \text { range }_{2} ; \ldots ; a_{n}=\alpha_{n} \text { range }_{p} .
$$

The initial population pool is stored as an $l \times n$ matrix, where 1 is the number of individuals in the initial population pool. Typically 1 is very large, since a pool of 500,000 individuals takes only about 5 minutes to generate and vastly improves the initial fitness.

\section{Selection of the First Generation}

The size of a generation is a user-defined variable, but typically about 10 individuals is appropriate. A first generation population is chosen by simply selecting a specified number of the most fit individuals in the population, as defined by the chosen fitness function. This first generation should already be relatively fit, particularly if the initial population pool it 


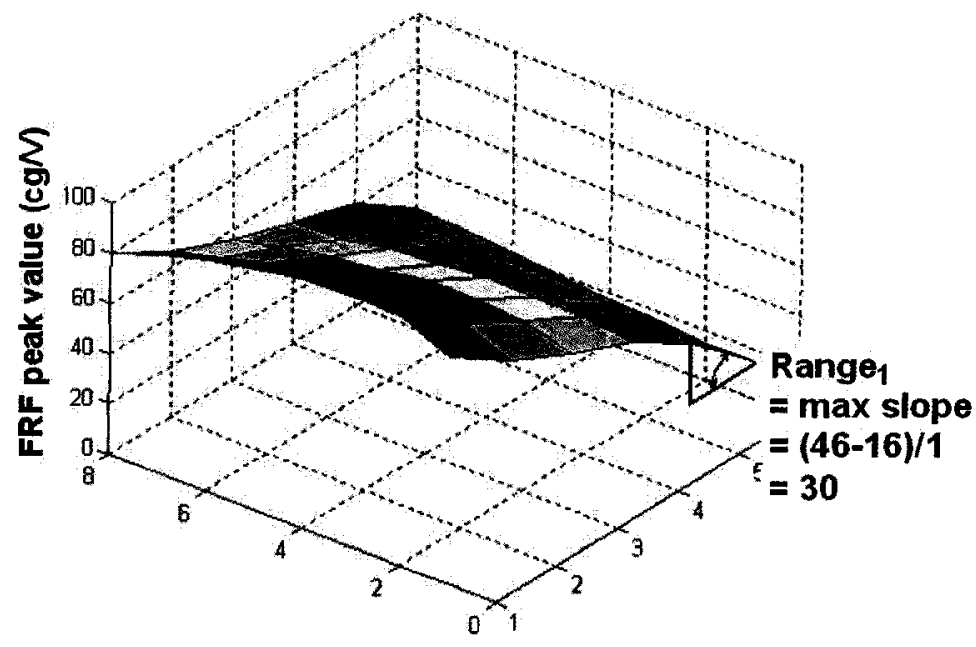

Figure 3.3: Calculation of Range Parameter $r_{1}$

is chosen from is large. A fit first generation greatly improves the subsequent convergence of the algorithm. The initial selection process consists of determining the fitness of every individual in the initial population pool, and selecting a pre-determined number of the most fit to compose the first generation. The fitness is defined as the inverse of the SRSS error. The algorithm identifies the individual with the highest fitness and records its index (position in the population pool array). It then sets the fitness to zero in order to avoid re-selecting the same individual repeatedly. Once the indices of the fittest individuals have been recorded, a new population matrix is composed from the initial population pool.

\section{Reproduction and Crossover}

Reproduction and crossover are two related processes that occur in nature and result in a mixing of genes for the next generation. In nature, reproduction usually occurs between a finite number of individuals in a population at each generation. In order to get the maximum possible variation and to ensure that fitness does not decrease for the next generation, in the surface fitting genetic algorithm, reproduction and crossover occur between all possible combinations of individuals, and also every individual is crossed with itself. This means that if there are $\mathrm{m}$ individuals in a population, there are $\mathrm{m}^{2}$ individuals following the 


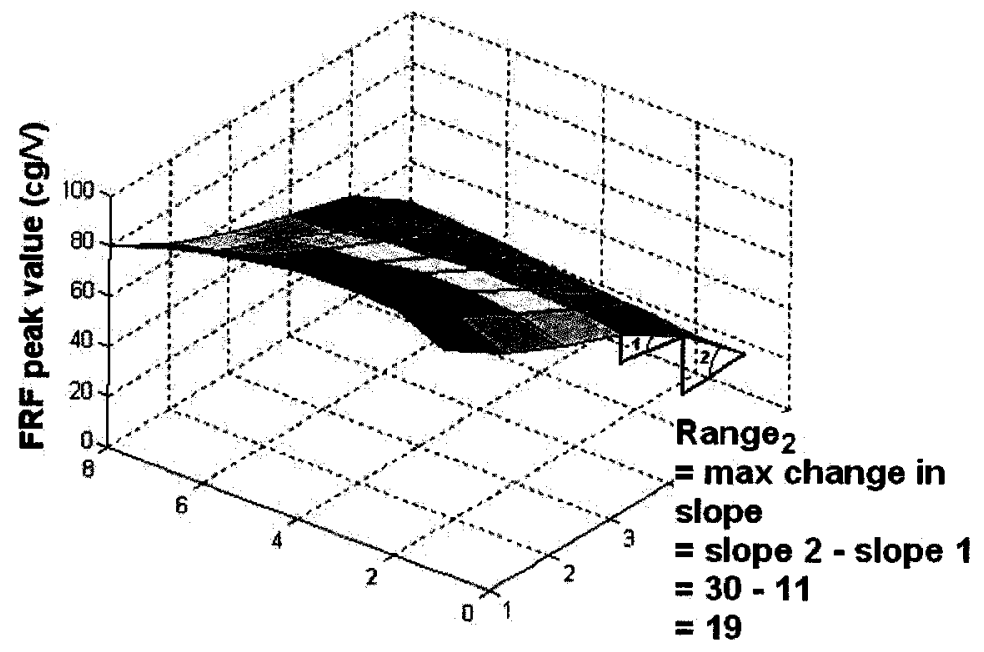

Figure 3.4: Calculation of Range Parameter $\mathrm{r}_{2}$

reproduction and crossover operation. The algorithm conducts reproduction and crossover in the same stage.

In nature, reproduction occurs on the organism level and crossover occurs on the cellular level, but both operations involve the transfer and mixing of genetic information. Crossover occurs during meiosis, the operation in which two gametes are produced from a regular cell. A gamete contains one of each chromosome, unlike a regular cell which contains two. This chromosome is composed of a portion of each of the two originals in the parent cell. During crossover, the chromosomes literally crossover and bind at a random position along the length. The first section of one chromosome forms part of one gamete, while the first portion of the other chromosome forms part of the other gamete. After the crossover point the chromosome portions that each gamete receives is switched. This crossing and the differences in the two original chromosomes are the only sources of variation amongst the gametes of an organism. The crossover in the genetic algorithm works in the same manner, except that it occurs immediately before reproduction. For two breeding individuals, a random point along the coefficient string is selected, and all the coefficients before that point are switched. Figure 3.5 shows an example of crossover with real strings for a second order polynomial. 


\section{$A=3.4 \quad 2.3 \quad 5.6 \quad-1.3 \quad 1.2 \quad-0.5$ \\ $B=0.91 .3\left[\begin{array}{llll}3.4 & -2.1 & 1.6 & -0.7\end{array}\right.$ \\ $A^{\prime}=3.4 \quad 2.3 \quad 3.4 \quad-2.1 \quad 1.6 \quad-0.7$ \\ $B^{\prime}=0.9 \quad 1.3 \quad 5.6 \quad-1.3 \quad 1.2 \quad-0.5$}

Figure 3.5: Example of Crossover at the Second Allele

Reproduction occurs next. In nature, this is the combination of two gametes to form a single zygote which contains a pair of each chromosome, one from each parent. Since the chromosome distribution was randomized during gamete production, the child effectively receives one of each parents chromosomes, subject to crossover, at random. Only one chromosome represents each individual in the genetic algorithm, so reproduction occurs at the chromosome level by a mixing process, rather than a selection of one chromosome or another at an organism level. Since the alleles in the genetic algorithm encode real numbers, instead of a roulette wheel process where one parent's allele or the other is selected for each locus at random, a random mixing process is used where each new allele is a randomly graded combination of that of the two parents:

$$
a_{i, \text { child }}=(\beta) a_{i, \text { parent } 1}+(1-\beta) a_{i, \text { parent } 2},
$$

where $\beta$ is a random number between 0 and 1 . Since the reproduction operation is a random mixing process, the crossover operation is not strictly necessary. Crossover was maintained because a roulette style (either-or) allele selection process was originally used for the algorithm (in which case crossover had a marked effect) and because the computational demand of the operation was very low.

\section{Mutation}

Although mutation is critical to introduce new information, it should be noted that it occurs with a small probability and also that most of the mutations are detrimental. The mutations that do not improve the solution are subsequently removed in the selection process. A very small number of beneficial mutations occur, but these contribute vital information to 
the final solution. The fact that most mutations are detrimental means that too high a mutation rate will slow convergence by detrimentally changing the fittest individuals of the population too often; however, too low a mutation rate will also slow convergence by not introducing sufficient mutations. Also, the mutation severity is a very important parameter in influencing convergence rate. If mutations are too large, they are far less likely to be beneficial because they overshoot the solution. Conversely, if mutations are too small, they only marginally improve the solution. Mutation chance and severity as they relate to the surface fitting algorithm are discussed below. The mutation operator developed specifically for this research is designed specifically for polynomial surface fitting:

$$
a_{\text {mutated }}=a_{\text {original }}+\left(\gamma \cdot \lambda \cdot \text { range }_{p}\right),
$$

where $\gamma$ is a random number between -1 and $1, \lambda$ is the mutation severity parameter, and the order of the range parameter depends on the order ( $p$ ) of the coefficient being mutated.

\section{Pre-Immigration Selection}

The initial selection process is exactly the same as the selection of the first generation population, except that instead of the selection being from a very large population pool, the selection is from the $\mathrm{m}^{2}$ individuals generated by reproduction and subsequently modified by mutation. The selection uses the inverse SRSS of the error fitness function, and $\mathrm{m}$ individuals are selected by the index recording procedure discussed earlier.

\section{Immigration}

Immigration is an infrequently discussed operator that can contribute the same addition of information at each generation effect as mutation. It can in some cases, even be used in place of a mutation operator [28]. Immigration inserts new individuals into a population at each generation in place of the individuals who remain unselected. These individuals can be generated in the same way as the individuals in the initial population, but this effect 
would be more easily accomplished by simply expanding the size of the initial population pool. Therefore, an alternative method that ensures a relatively high fitness is normally used.

In the surface fitting genetic algorithm, immigration was accomplished by replacing the $\left(\mathrm{m}^{2}-\mathrm{m}\right)$ unselected individuals at each generation with newly generated individuals whose characteristics (coefficients in this case) were randomly selected from the $\mathrm{m}$ fittest members of the population. The $\mathrm{m}$ best variations of each allele were combined into a pool. The $\left(\mathrm{m}^{2}-\mathrm{m}\right)$ immigrating individuals were then generated by randomly selecting one allele variation from each pool. Each allele in the immigrating individuals was randomly modified by up to $+/-10 \%$. This vastly improved the fitness of the pool by replacing the least fit individuals in a generation with many possible combinations of variations of alleles from the most fit. It was therefore similar to a second reproduction process. Unlike mutation, the immigrating individuals were not based on the initial population or a range parameter based on data scatter. The immigration operator therefore complemented the mutation operator, contributing separately but effectively to convergence rate. Although the genetic algorithm could have functioned without the immigration operator, a combination of mutation and immigration yielded dramatically better results, as shown by Figure 3.6.

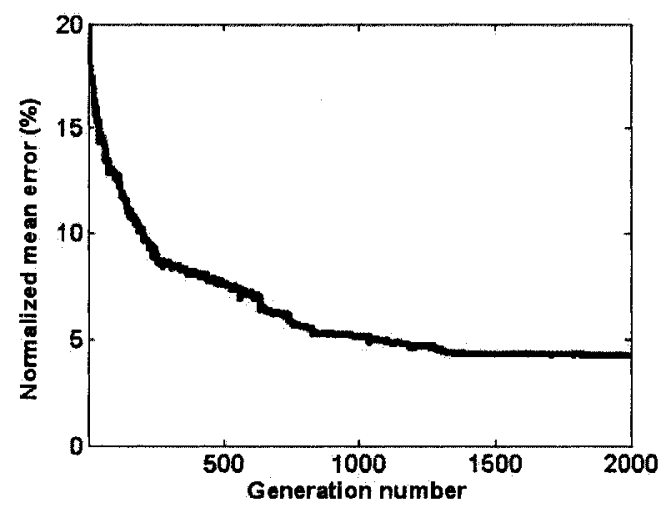

(a) With Immigration

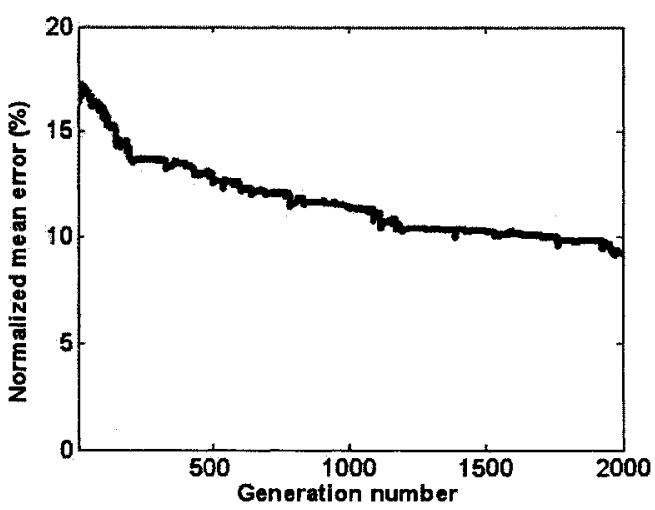

(b) Without Immigration

Figure 3.6: Comparison of Convergence Rate With and Without the Immigration Operator 


\section{Re-selection and Repetition}

Following reproduction and crossover, mutation, pre-immigration selection, and immigration, a post-immigration selection is conducted to assemble a new size $\mathrm{m}$ population. This differs from the pre-immigration selected population only if one or more of the immigrating individuals are more fit than those previously selected. As with pre-immigration selection, this process uses the inverse SRSS of the error fitness function, and the $\mathrm{m}$ individuals are selected by the index recording procedure discussed above.

This group of post-immigration selected individuals forms the next generation, and all steps of the algorithm repeat until the algorithm is terminated.

\section{Algorithm Termination}

Two possible criteria for algorithm termination are used in the genetic algorithm. One or both can be used for a surface fitting process. The first is simply the fitness or error measurement. The algorithm can be ordered to terminate when the error drops below a sufficiently low level (such as when the normalized mean error drops below 5\%). This approach is reasonable, but it does not take into account the suitability of the polynomial order or size of data set. For some data sets, a particular polynomial order will never achieve a sufficiently low error, and such an algorithm termination criteria will result in an infinite loop. Also, it may not be initially clear what magnitude of error should be achievable for a particular data set. For these reasons, a second termination criteria that could be used either independently or in conjunction with the error measurement was required.

The number of generations is an excellent termination criteria, because given an average time per generation, the algorithm can be run for a specific amount of time, and the algorithm can obtain the best possible solution within that time. Since an error vs. generation plot is generated for each run, convergence can be assessed from the slope of the error vs. generation line. If no further reduction in error is achieved after several hundred generations, the algorithm can be considered to have obtained the best possible polynomial surface fit using the chosen polynomial order and data set. If the fitness was still improving 
at algorithm termination, it can be re-initiated with a larger number of generations. Table 3.3 shows the approximate time required by the algorithm per generation by polynomial order. This time is heavily influenced by computer speed, and this Table is consistent with the processing power of a $2000 \mathrm{MHz}$ Pentium personal computer.

Table 3.3: Approximate Number of Generations per Second by Polynomial Order on a $2000 \mathrm{MHz}$ Pentium PC

\begin{tabular}{cc}
\hline \hline Polynomial Order & Approximate Number of Generation per Second \\
1st & 500 \\
2nd & 200 \\
3 rd & 70 \\
4 th & 25 \\
5 th & 10 \\
6 th & 5 \\
7 th & 3 \\
8 th & 2 \\
9 th & 1.5 \\
\hline
\end{tabular}

\subsubsection{Factors Affecting Convergence}

Several user defined variables affect the convergence of the algorithm. The polynomial order and population pool size are determined from the variability in the data set. Widely scattered FRF peak magnitudes require higher polynomial orders and, in order to achieve good convergence rates, high initial population pool sizes. Mutation probability and severity are also important factors in achieving a successful surface optimization.

\section{Polynomial Order}

Using an appropriate polynomial order is essential to obtaining a good polynomial surface optimization. In general, the error decreases with increasing polynomial order. However, it may be possible to obtain an optimal polynomial with a lower order, eliminating the need for additional computation. With the first and third modes from the flexible fin vibration, 
it was convenient to start with a low polynomial order and increase the order until the error decreased to an acceptable level after a certain number of generations. This strategy should be pursued when searching for a correct polynomial order. If the fully converged error does not substantially decrease with a polynomial order increase, the lower polynomial order is suitable, unless both polynomial orders result in a very high error. In this case, an optimization using the next higher order should be attempted, however it can arise with very scattered data that a prohibitively high order polynomial may be required to obtain a sufficiently low error. In the case of the piezoelectric actuator FRF peak values, this occurred for the second mode. The solution was to divide the range into smaller subregions and optimize a polynomial over each separately. The error associated with increasing polynomial order for the third mode after 2000 generations is shown in Table 3.4. Since the transition from an 8 th to 9 th order polynomial did not significantly reduce the error, an 8 th order polynomial was selected to reduce computational complexity.

Table 3.4: Effect of Polynomial Order on Error

\begin{tabular}{cccc}
\hline \hline Polynomial Order & SRSS error $(\mu \mathrm{g} / \mathrm{V})$ & Normalized Mean Error $(\%)$ & R-square \\
4 th & 740 & 27 & 0.47 \\
5 th & 680 & 22 & 0.54 \\
6 th & 460 & 18 & 0.63 \\
7 th & 320 & 14 & 0.72 \\
8 th & 240 & 10 & 0.86 \\
9 th & 220 & 9 & 0.88 \\
\hline
\end{tabular}

\section{Population Pool Size}

The size of the initial population pool proved to be one of the most important factors in optimization of the polynomial surface. A large population pool results in a much higher first generation fitness, which substantially improved the subsequent convergence. Since the algorithm is in fact a random search followed by natural selection to improve the solution, a more successful random search increases the success of the natural selection process, 
particularly with a large or widely scattered data set. Although the genetic algorithm should eventually obtain the optimal polynomial even with a small population pool, in the case of a high order polynomial with many data points, the convergence time could be prohibitively long resulting in an inferior solution for a given number of generations. It is relatively simple and efficient to generate a very large population pool (50,000 or more individuals in under a minute) from which to draw the first generation. The effect of population pool size on convergence rate for the first mode after 2000 generations is shown in Table 3.5.

Table 3.5: Effect of the Initial Population Pool Size on Convergence

\begin{tabular}{cccc}
\hline \hline Population Pool Size & SRSS error $(\mu \mathrm{g} / \mathrm{V})$ & Normalized Mean Error $(\%)$ & R-square \\
100 & 57 & 12 & 0.87 \\
1,000 & 48 & 10 & 0.93 \\
10,000 & 28 & 6 & 0.97 \\
50,000 & 21 & 4 & 0.98 \\
\hline
\end{tabular}

\section{Mutation Probability}

Mutation enhances the speed with which a genetic algorithm can find a near-optimum solution by introducing new alleles into the population, allowing the final solution to incorporate information that was not included in the initial population. Although mutation is critical to introduce new information, it should be noted that it occurs with a small probability and also that most mutations are detrimental in that they usually reduce the fitness of an individual. These detrimental mutations are subsequently selected out in the selection process, while the infrequent beneficial mutations are absolutely vital to enhancing the convergence speed. Since most mutations are detrimental, too high a mutation rate slows convergence by reducing population fitness. However, too low a mutation rate also slows convergence by failing to induce sufficient mutations. The mutation chance is defined as the probability, applied separately to each coefficient (allele) in each coefficient set (individual), with which a mutation occurs. The effect of mutation probability on convergence rate for the first 
mode after 2000 generations is shown in Table 3.6. A sufficiently large number of generations should eventually yield the same error for all cases as in the $5 \%$ case. However, a very high mutation probability would dramatically slow convergence, or in a worst case, result in non-convergence. A $5 \%$ mutation probability was used for polynomial surface fitting.

Table 3.6: Effect of Mutation Probability on Convergence

\begin{tabular}{cccc}
\hline \hline Mutation Probability (\%) & SRSS error $(\mu \mathrm{g} / \mathrm{V})$ & Normalized Mean Error (\%) & R-square \\
1 & 55 & 11 & 0.89 \\
2 & 50 & 9 & 0.92 \\
5 & 21 & 4 & 0.98 \\
10 & 24 & 5 & 0.96 \\
25 & 30 & 6 & 0.94 \\
\hline
\end{tabular}

\section{Mutation Severity}

In biological science there are many types of mutations based on the replication of DNA, such as substitutions, where an allele replaces another, or deletions, where an allele is removed and a phase shift occurs. For the surface fitting algorithm, the purpose of mutation was to introduce a chance of a small variation in the real-valued alleles at each generation. The severity of this small variation was absolutely critical not only to ensure a reasonable convergence rate, but to achieve convergence at all.

The severity of mutation has a moderate effect on the convergence rate. If mutations are too large, they are far more likely to be detrimental. Conversely, if mutations are too small, they are unlikely to produce a significant favourable change. The effect of mutation severity on convergence rate for the first mode after 2000 generations is shown in Table 3.7. The optimal mutation severity of $\lambda=0.1$ was used for polynomial surface fitting. 
Table 3.7: Effect of Mutation Severity on Convergence

\begin{tabular}{cccc}
\hline \hline Mutation Severity $(\lambda)$ & SRSS error $(\mu \mathrm{g} / \mathrm{V})$ & Normalized Mean Error (\%) & R-square \\
0.01 & 23 & 5 & 0.96 \\
0.05 & 22 & 5 & 0.97 \\
0.1 & 21 & 4 & 0.98 \\
0.5 & 38 & 8 & 0.95 \\
1 & 83 & 17 & 0.77 \\
\hline
\end{tabular}

\subsection{Polynomial Surface Fitting Results}

Convergence was achieved easily for the first and third modes using 3rd and 8th order polynomial surfaces. The second mode proved to be a much more challenging case, and even a 9 th order polynomial resulted in an unacceptably high error. Thus, rather than a single polynomial surface, four neighbouring sub-surfaces were used. The combination of these subsurfaces described the FRF peak values over surface of the fin very well.

\subsubsection{First Mode}

The scatter of the peak FRF magnitudes for the first mode was minor, and generation of the best polynomial surface for the first mode was therefore straightforward. A 3rd order polynomial was sufficient to obtain a low error. The discrete FRF peak values (with shading but no surface), and the corresponding polynomial surface fit for the first mode are shown in Figure 3.7. As expected from the mode shapes shown in Figure 2.1, the peak FRF magnitudes were concentrated at the fin root closest to the node line running along the root from the leading edge to the trailing edge. The FRF magnitudes measured at the accelerometer slowly decreased as the actuator was placed further from the root.

\subsubsection{Second Mode}

The second mode FRF peak values presented a particular challenge for obtaining the best polynomial surface. Although the error decreased with increasing polynomial order, even a 


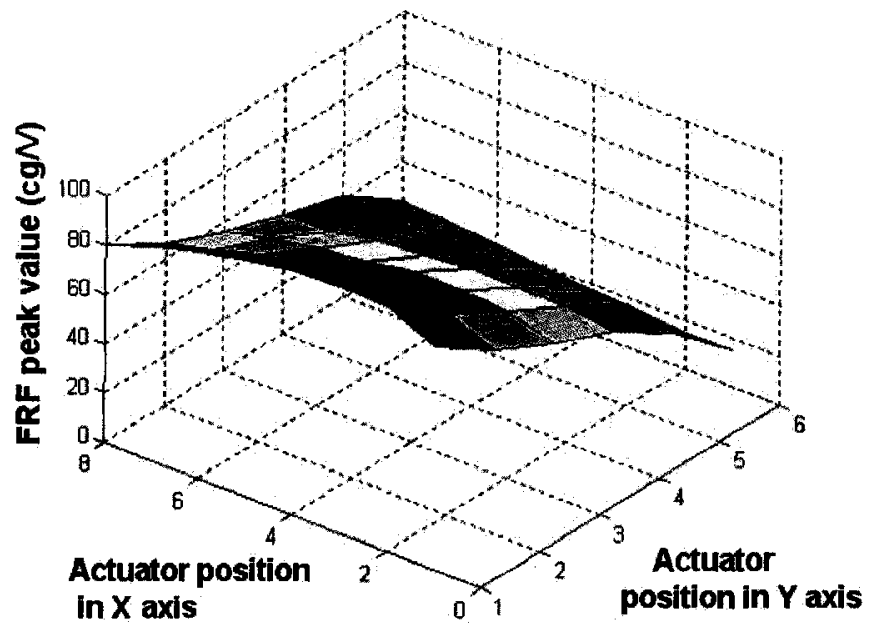

(a) Discrete FRF Peak Magnitudes

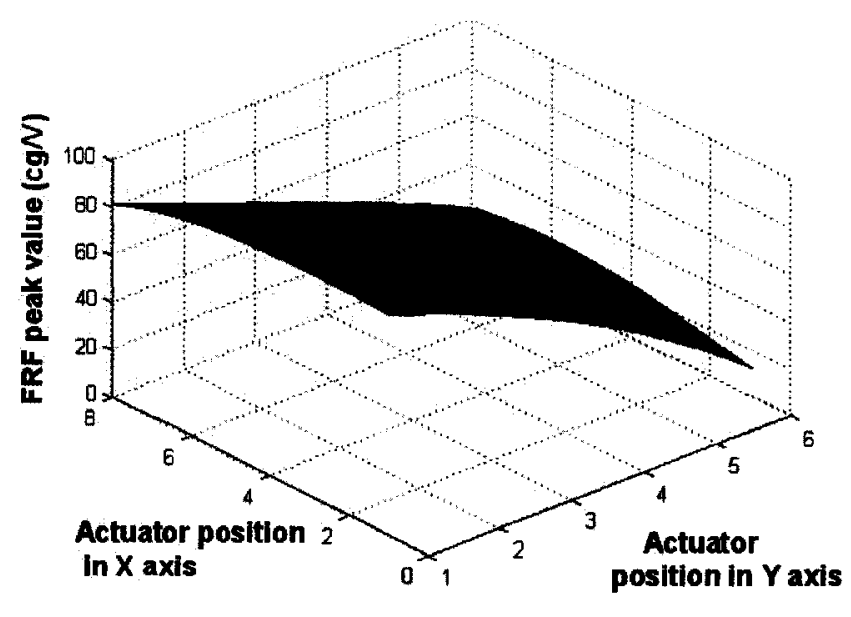

(b) Best 3rd Order Surface Fit

Figure 3.7: Comparison of the Discrete FRF Peak Magnitudes and the Best 3rd Order Polynomial Surface for the First Mode

9th order polynomial resulted in a normalized mean error of $38 \%$ and an R-square value of 0.63. The discrete FRF peak values (with shading but no surface), and this corresponding 9th order polynomial surface are shown in Figure 3.8. This was evidently not a sufficiently good surface fit. This resulted from the fact that the second mode had too much variation in the peak values to be adequately represented by a single 9 th order polynomial. Increasing the polynomial order would eventually produce a better optimized surface, but it would be prohibitively complicated to fit such a surface, let alone manipulate it. This problem was solved by subdividing the actuator test area into four separate neighbouring sub-areas, over each of which a polynomial sub-surface was optimized. As expected from the mode shapes shown in Figure 2.1, the peak FRF magnitudes were concentrated at the corners of the actuator test area, as distant as possible from the central torsional node line running from root to tip.

\section{Neighbouring Polynomial Sub-Surfaces}

When attempting to match challenging data sets to two-dimensional curves, a possible solution is to use splines. This divides the problem into sub-problems whereby a different curve 


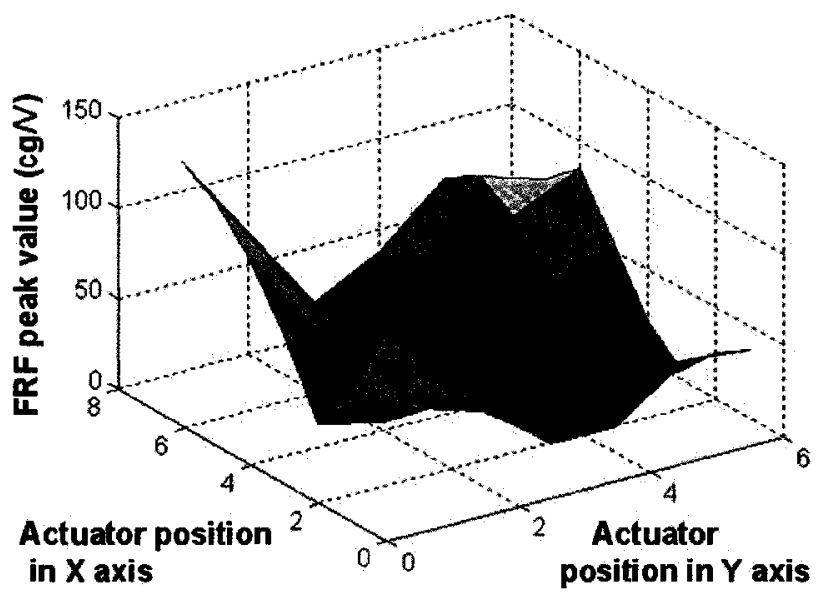

(a) Discrete FRF Peak Magnitudes

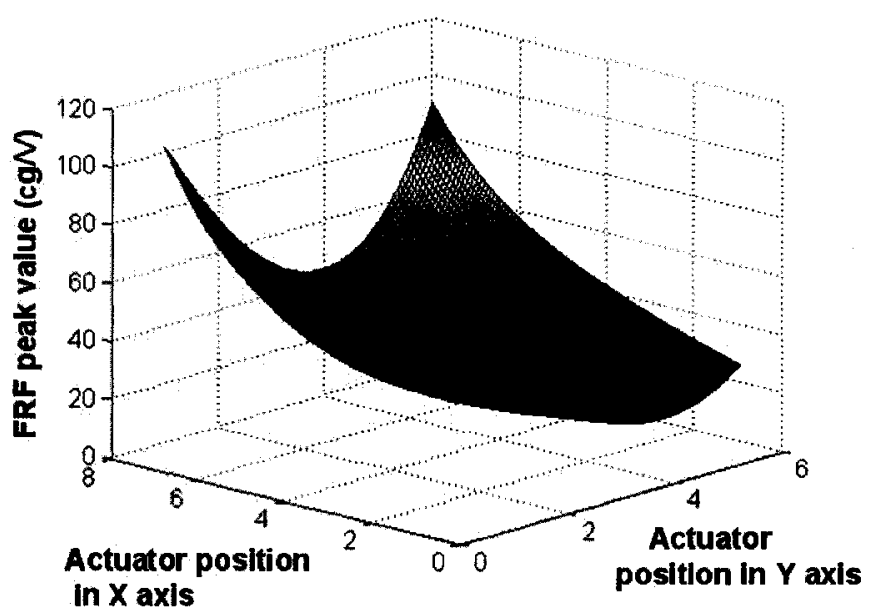

(b) Best 9th Order Fit Using a Single Surface

Figure 3.8: Comparison of the Discrete FRF Peak Magnitudes and the Best Single 9th Order Polynomial Surface for the Second Mode

represents the data between each point. With a spline, continuity is normally established by fixing the data value and first derivative of the two curves at each point. The method of splines can be extended to polynomial surface fitting. However, in this case it is not necessary to fix neither the data value nor slope provided that the sub-surfaces cover the entire region. In fact, it would add unnecessary complexity. Continuity at the junctions is not necessary to be established between the sub-surfaces because optimization of the actuator position requires a FRF peak value at any point but not necessarily continuous polynomial surfaces. Along the junctions, the mean FRF peak values can be used. The actuator test area was therefore divided into four sub-areas as shown in Figure 3.9 over which corresponding sub-surfaces were optimized. The division of the problem into 8th order sub-surfaces resulted in low error for all the individual sub-surfaces, as shown in Table 3.8. These sub-surfaces are shown in Figures 3.10 and 3.11. Note that the sub-surfaces share common points and there is a coordinate transformation so that all the sub-surfaces use $\mathrm{X}$ and $\mathrm{Y}$ coordinates between 1 and 4 . The combination of these sub-surfaces over the entire actuator test area provided an overall optimal polynomial surface, which is shown in Figure 3.12. 


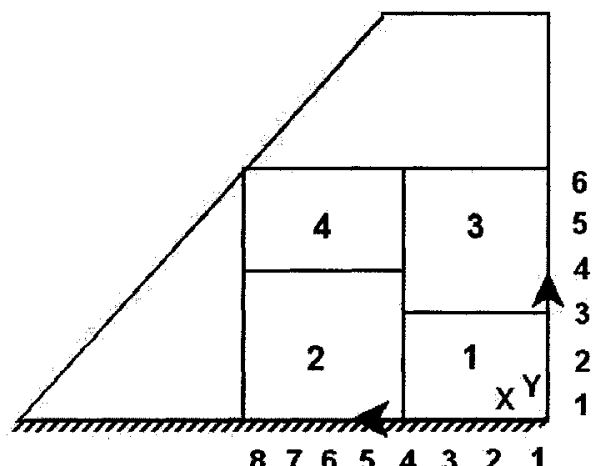

Figure 3.9: The Actuator Test Area Subdivisions

Table 3.8: Best Single 9th Order Surface and Sub-surface Coordinates and Error for the Second Mode

\begin{tabular}{ccccc}
\hline \hline Surface & X-Coordinates & Y-Coordinates & Norm. Mean Error (\%) & R-square \\
Best 9th Order & N/A & N/A & 38 & 0.63 \\
Sub-surface 1 & $1-4$ & $1-3$ & 8 & 0.95 \\
Sub-surface 2 & $4-8$ & $1-4$ & 8 & 0.95 \\
Sub-surface 3 & $1-4$ & $3-6$ & 10 & 0.93 \\
Sub-surface 4 & $4-8$ & $4-6$ & 6 & 0.97
\end{tabular}

\subsubsection{Third Mode}

The surface fitting for the third mode with the complete set of positions was more complex than the first mode, but was still relatively straightforward. An 8th order polynomial was necessary to obtain $10 \%$ error, and a 9 th order polynomial did not show substantial improvement. If required, the error could be further reduced by sub-dividing the region using neighbouring sub-surfaces as described for the second mode. The discrete FRF peak values (with shading but no surface), and the corresponding polynomial surface fit for this mode are shown in Figure 3.13. As expected from the mode shapes shown in Figure 2.1, the peak FRF magnitudes were concentrated at the fin root closest to the node line running along the root from the leading edge to the trailing edge, and at the central node line running from the leading edge to the trailing edge midway along the fin from root to tip. 


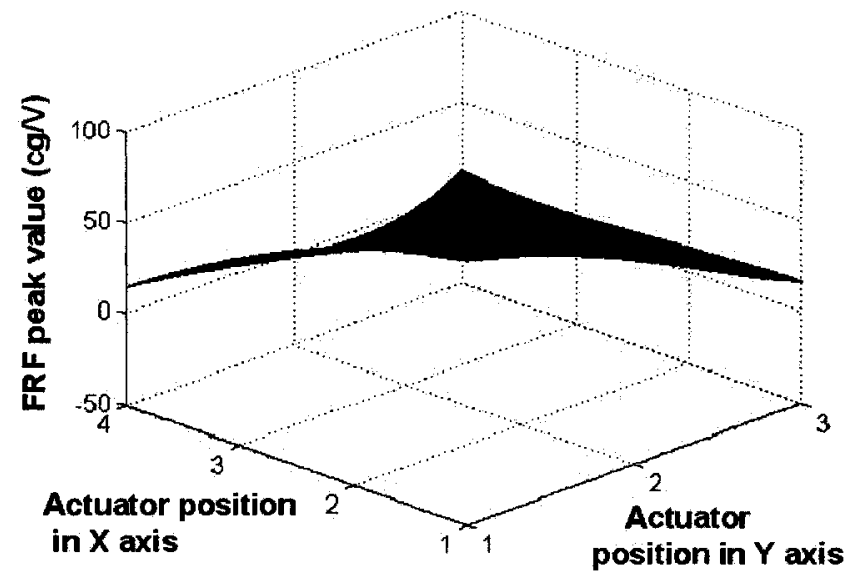

(a) Sub-surface 1

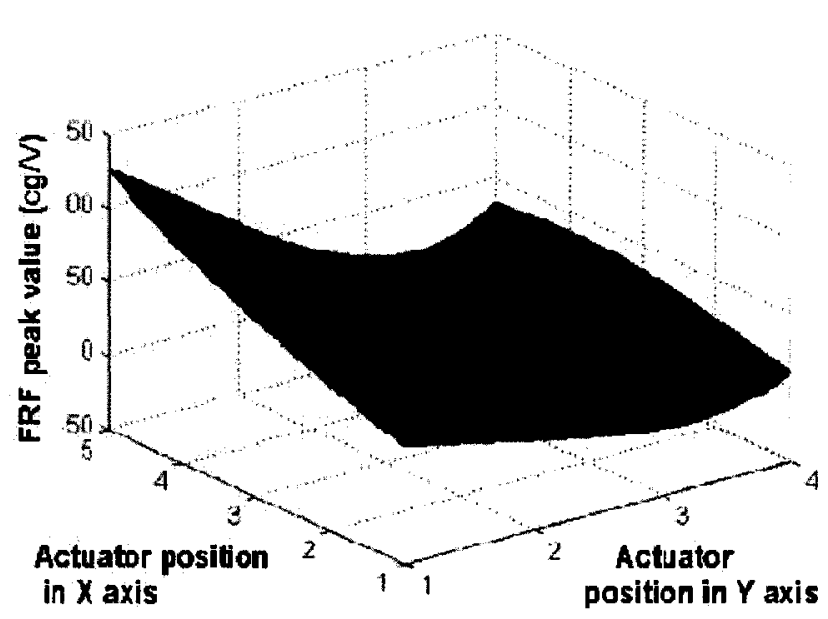

(b) Sub-surface 2

Figure 3.10: The First (a) and Second (b) Sub-surfaces for the Second Mode

The FRF magnitudes measured at the accelerometer decreased as the actuator was placed further from these node lines.

\subsection{Genetic Algorithm for Actuator Positional Optimization}

A second genetic algorithm was developed to optimize the position of the single actuator pair on the flexible fin surface. This genetic algorithm was substantially simpler than that developed for polynomial surface fitting, and no problems were encountered in its application. The genetic algorithm maximized the overall peak FRF magnitude recorded at the accelerometer for the combination of modes as given by the fitness function described below. This algorithm used a population of individuals containing two alleles of continuous variables representing $\mathrm{x}$ and $\mathrm{y}$ coordinates within the actuator test area. Each individual therefore represented an actuator position, and the most fit individual (that with the highest overall peak FRF magnitude recorded at the accelerometer), was the optimum actuator position for maximization of actuator authority for control of acceleration. A similar fitness function was used to optimize the actuator position for displacement control. 


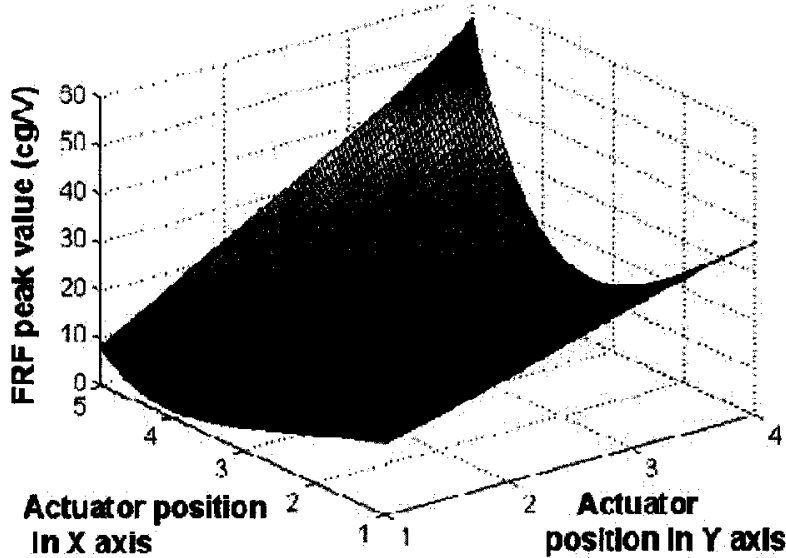

(a) Sub-surface 3

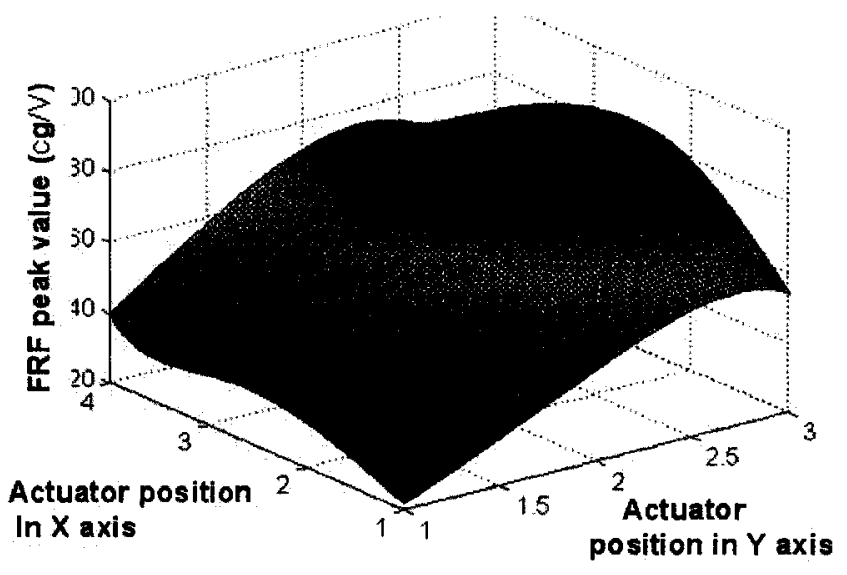

(b) Sub-surface 4

Figure 3.11: The Third (a) and Fourth (b) Sub-surfaces for the Second Mode

\subsubsection{Fitness Function for Positional Optimization}

The fitness function was determined from a weighted summation of the polynomial surfaces. Since the second mode was discontinuous at the sub-area division, this resulted in a discontinuous overall fitness function. However, since the alleles were composed of continuous variables ( $\mathrm{x}$ and $\mathrm{y}$ coordinates), it was extremely unlikely for one to fall directly on the subdivision. If one did, the mean value along the division was used. The weighting factors are dependent on the desired vibration control emphasis over each of the first three modes, and directly influence the optimum actuator location. Since FRF is a direct normalized measure of acceleration response, the fitness function used for acceleration control was

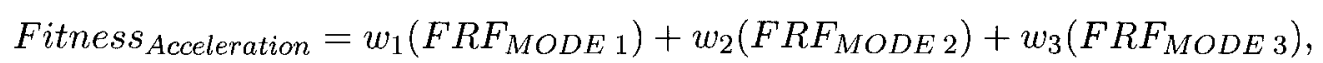

where $w_{1}, w_{2}$, and $w_{3}$ are the weighting factors on the first, second, and third modes, respectively.

Similarly for displacement control, considering harmonic motions, the fitness function was taken to be the sum of the weighted individual modal peak FRF values divided by the square of the fundamental frequencies for each mode. The objective was therefore to maximize: 


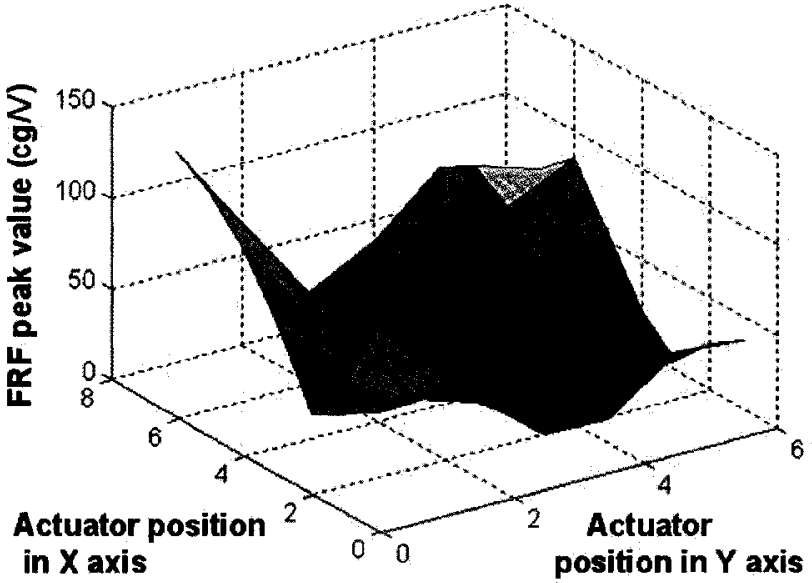

(a) Discrete FRF Peak Magnitudes

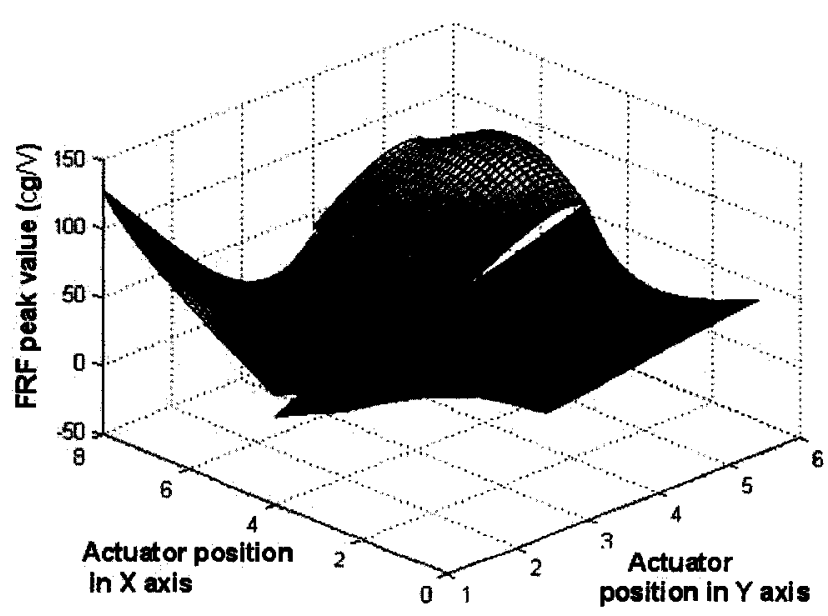

(b) Combined Sub-surface Fit

Figure 3.12: Comparison of the Discrete FRF Peak Magnitudes (a) and the Combined Subsurfaces (b) for the Second Mode

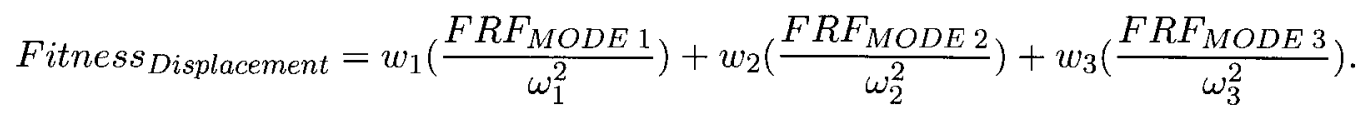

\subsubsection{Genetic Algorithm Steps}

The genetic algorithm for single actuator pair positional optimization was substantially more simple than that for polynomial surface fitting. No initial population pool or range parameters were required since the alleles ( $\mathrm{x}$ and $\mathrm{y}$ coordinates) were constrained by the geometry of the fin. Reproduction and mutation were simple operations and the computational requirements were very low, both in terms of generations to convergence (under 100 ), and computation time (several seconds). The algorithm terminated after a specified number of generations, or when there was no improvement in solution after 10 generations. The actuator position optimization algorithm incorporates the following steps:

1. An initial population of 10 individuals is formed by randomly selecting $\mathrm{x}$ and $\mathrm{y}$ values for each within the actuator test area.

2. The individuals in the population are bred with every other individual (including 


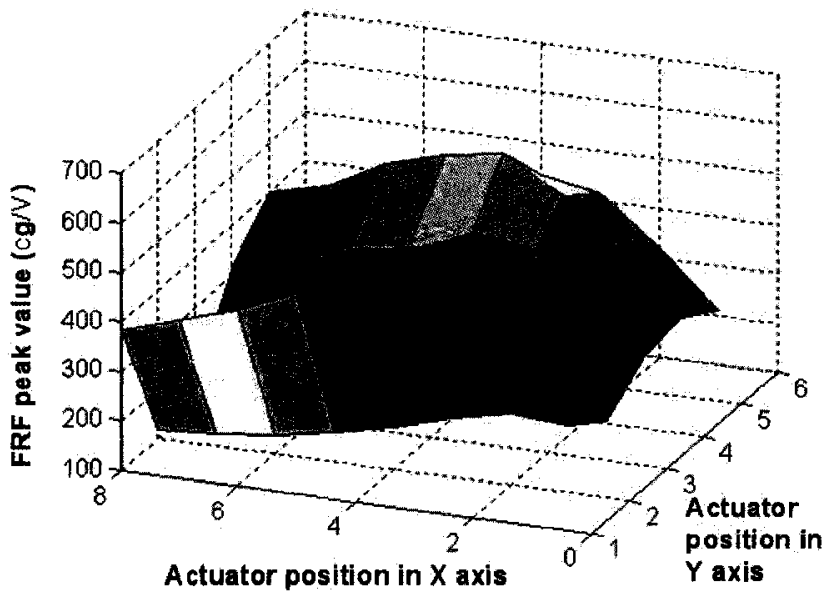

(a) Discrete FRF Peak Magnitudes

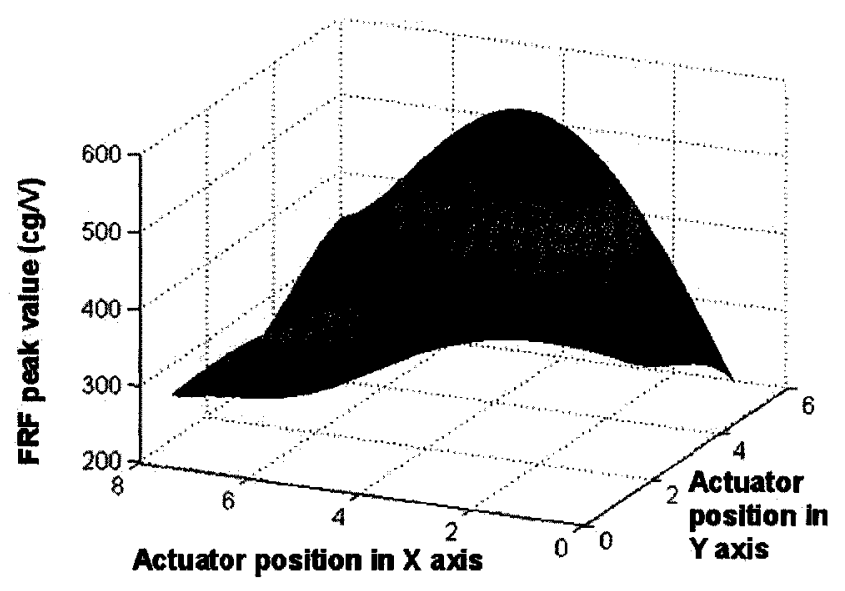

(b) Best 8 th Order Surface Fit

Figure 3.13: Comparison of the Discrete FRF Peak Magnitudes and the Best 8th Order Polynomial Surface for the Third Mode

themselves in a self-cross) to form 100 individuals. The breeding is a random mixing process, where for every combination, the child's allele is a random value between the values of the two parents' alleles.

3. Mutation is applied, giving each allele in the population a $5 \%$ chance of randomly changing in value ( $\mathrm{x}$ or $\mathrm{y}$ coordinate) by up to 0.5 .

4. The fitness is calculated for each individual, and the algorithm is checked for termination. If the algorithm does not terminate, the next generation is formed by the 10 fittest individuals.

5. Reproduction, mutation, and selection are repeated until the algorithm terminates.

\subsubsection{Positional Optimization}

Several cases were considered for positional optimization of the pair of actuators for vibration control, including individual control of each mode and multi-modal acceleration and displacement control. The algorithm is capable of optimizing for other cases using the 
weighting factors in the fitness function. Note that positions within $20 \mathrm{~mm}$ of the fin root were excluded from the optimization due to the potential for interference with the attachment. This was not a requirement for the configuration optimization discussed in chapter 4 , and led to some discrepancies in results.

\section{First Mode}

The optimum position for a pair of actuators for the first mode was determined by using the fitness function in Equation 3.17 with weighting factors $w_{1}=1$ and $w_{2}=w_{3}=0$. Since there is no contribution from other modes, the optimum position for acceleration and displacement control are effectively the same, since a position of maximum fitness would remain so even if divided by the square of the corresponding fundamental modal frequency as in Equation 3.18. The optimum position using this criteria was at $x=55 \mathrm{~mm}, y=20 \mathrm{~mm}$, as shown in Figure 3.14. This corresponds to a positioning as close as possible to the fin root fairly close to the trailing edge. This position is consistent with the peak FRF magnitude plots and with the fin nodes.

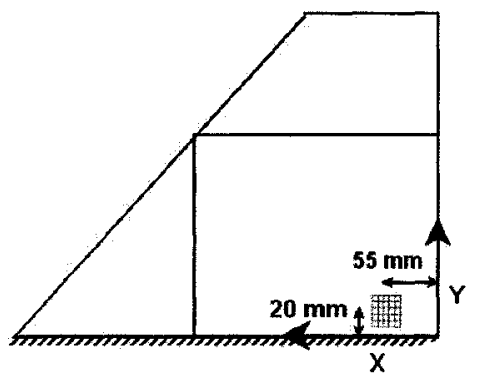

Figure 3.14: The Optimum Actuator Pair Position for the First Mode

\section{Second Mode}

As with the first mode, the optimum position for a pair of actuators for the second mode was determined by using the fitness function in Equation 3.17 with weighting factors $w_{2}=1$ and $w_{1}=w_{3}=0$. The optimum position using this criteria was at $\mathrm{x}=190 \mathrm{~mm}, \mathrm{y}=120 \mathrm{~mm}$, as shown in Figure 3.15. This corresponds to a positioning as far as possible from the torsional 
node line of the fin that runs from root to tip at mid-chord from the leading edge to the trailing edge. This is consistent with the peak FRF magnitude sub-surface plots.

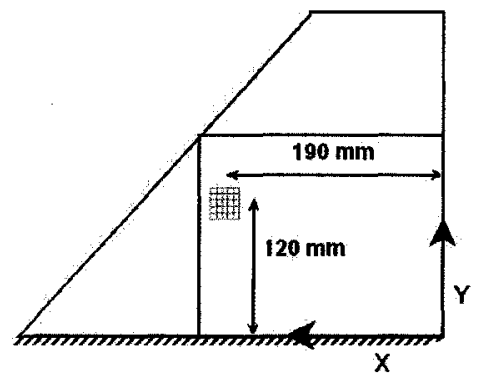

Figure 3.15: The Optimum Actuator Pair Position for the Second Mode

\section{Third Mode}

As with the first and second modes, the optimum position for a pair of actuators for the third mode was determined by using the fitness function in Equation 3.17 with weighting factors $\mathrm{w}_{3}=1$ and $\mathrm{w}_{1}=\mathrm{w}_{2}=0$. The optimum position using this criteria was at $\mathrm{x}=110 \mathrm{~mm}$, $\mathrm{y}=145 \mathrm{~mm}$, as shown in Figure 3.16. This corresponds to a positioning as on the second bending node line of the fin about a quarter of the way from trailing edge to leading edge. Positions near the fin root also yielded high actuation authority, as there corresponded more closely with the first bending node line. These positions are consistent with the peak FRF magnitude plots.

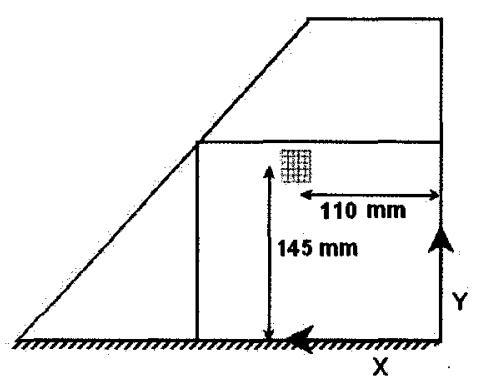

Figure 3.16: The Optimum Actuator Pair Position for the Third Mode 


\section{Acceleration Control}

The optimum position for a pair of actuators for multi-modal control of acceleration using even weighting factors $\left(w_{1}=w_{2}=w_{3}=1\right)$ in Equation 3.17 was a trade off between the positions for individual modal control. Each mode independently influenced the position of the pair of actuators. Since the peak FRF magnitudes were much higher for the second and particularly the third mode, these modes dominated for acceleration control and most heavily influenced the optimum position. This position was at $\mathrm{x}=160 \mathrm{~mm}, \mathrm{y}=125 \mathrm{~mm}$, as shown in Figure 3.17.

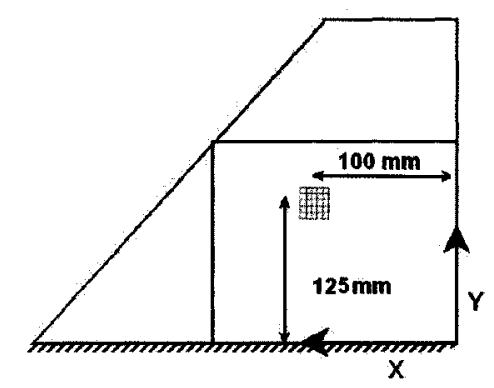

Figure 3.17: The Optimum Actuator Pair Position for Acceleration Control

\section{Displacement Control}

The optimum position for a pair of actuators for multi-modal control of displacement using even weighting factors $\left(\mathrm{w}_{1}=\mathrm{w}_{2}=\mathrm{w}_{3}=1\right)$ in Equation 3.18 was again a trade off between the positions for individual modal control. Each mode independently influenced the position of the pair of actuators. Since the fundamental frequencies were much lower for the second and particularly the first mode, these modes dominated for displacement control and most heavily influenced the optimum position. This position was at $\mathrm{x}=25 \mathrm{~mm}, \mathrm{y}=20 \mathrm{~mm}$, as shown in Figure 3.18.

\section{Other Possible Cases}

An infinite number of possible optimum actuator positions are possible, depending on the values of the weighting factors. These factors allow emphasis to be placed on particular 


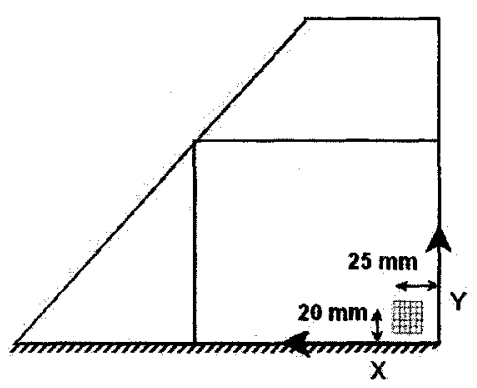

Figure 3.18: The Optimum Actuator Pair Position for Displacement Control

modes, in order to achieve a particular vibration control objective. For example, to highlight the importance of torsional vibration control, the weighting factor $\mathrm{w}_{2}$ could be increased. Two cases other than acceleration, displacement, or individual modal control were considered. One used weighting factors which equalized the mean peak FRF magnitudes of all modes for the fitness function, while the other used weighting factors which equalized the maximum peak FRF magnitudes of all modes. These cases are shown along with the cases for individual modal control and acceleration and displacement control in Table 3.9. The combined polynomial surfaces with weighting factors to equalize the maximum peak FRF magnitudes is shown in Figure 3.19.

Table 3.9: Optimum Actuator Position with Different Weighting Factors

\begin{tabular}{cccccc}
\hline \hline Weighting Case & $\mathrm{w}_{1}$ & $\mathrm{w}_{2}$ & $\mathrm{w}_{3}$ & $\mathrm{x}$ position $(\mathrm{mm})$ & y position $(\mathrm{mm})$ \\
First Mode & 1 & 0 & 0 & 55 & 20 \\
Second Mode & 0 & 1 & 0 & 190 & 120 \\
Third Mode & 0 & 0 & 1 & 110 & 145 \\
Acceleration Control & 1 & 1 & 1 & 160 & 125 \\
Displacement Control & 1 & 1 & 1 & 25 & 20 \\
Weighted to Equalize Mean FRF & 0.82 & 1 & 0.34 & 185 & 115 \\
Weighted to Equalize Maximum FRF & 1 & 0.35 & 0.23 & 135 & 110
\end{tabular}




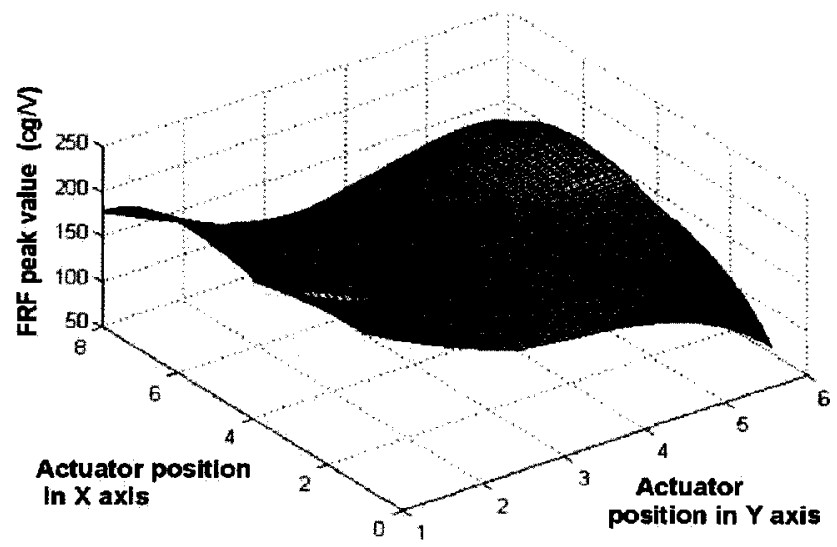

Figure 3.19: Combined Multi-modal Polynomial with Weighting Factors to Equalize Mean FRF Magnitudes 


\section{Chapter 4}

\section{Multiple Actuator Grouping Optimization}

This chapter describes the optimization of the configuration of a pre-determined number of piezoelectric actuator pairs within the actuator test area of the fin for either individual control of the first three modes or for control of a combination of the modes simultaneously. The actuation authority for each configuration is determined, allowing the number of actuators to be minimized for a specific vibration control requirement. Although the actuation authority always increases for each additional actuator, often this increased authority is minimal and the configuration efficiency suffers. The overall approach is described and then the procedure and results are discussed in detail.

\subsection{Approach: Direct Peak FRF Maximization}

For multiple actuator activation, the objective was to determine the optimum configuration of a pre-determined number of actuator pairs to activate from the 48 possible actuator positions on the fin. In this case it was much simpler to develop a fitness function as compared with the single actuator problem due to its discrete nature. No surface fitting of FRF results was required, and the configuration optimization could proceed by a simple direct maximization of FRF peak magnitudes. A genetic algorithm was employed to directly optimize the configuration from the fitness function. 


\subsection{Frequency Response Function Results}

This section presents the FRF peak magnitude results measured at the first accelerometer using the finite element model for each of the first three fundamental frequencies. These results will later be used to optimize the configuration of multiple actuators on the flexible fin for vibration control of acceleration or displacement using a genetic algorithm. The results are similar to those obtained in Chapter 3, however, they are based on a different finite element model of the fin. In this case all 48 actuator pairs are present for all measurements but only one is activated in turn, whereas only one actuator pair was fixed to the fin for each measurement in the case of single actuator positional optimization. The x-coordinates of the actuators are numbered 1-8 and the y-coordinates are numbered 1-6, since there were 8 and 6 possible positions in the $\mathrm{x}$ and $\mathrm{y}$ directions, respectively, as shown in Figure 2.5.

\subsubsection{First Mode}

The first vibrational mode of the fin, or first bending mode, was excited at a frequency of approximately $17 \mathrm{~Hz}$. The peak FRF values at the reference point for the first mode are plotted vs. the centroid of actuator position in Figure 4.1. Note that for vibration control of the first mode, the optimum actuator position should be near the root of the fin, particularly at the trailing edge. This is consistent since maximum control authority for the first mode should be achieved by placing actuators as close as possible to the node line that runs along the fixed fin root from leading edge to trailing edge.

\subsubsection{Second Mode}

The second vibrational mode of the fin, or first torsional mode, was excited at a frequency of approximately $47 \mathrm{~Hz}$. The peak FRF values at the reference point for the second mode are plotted vs. the centroid of actuator position in Figure 4.2. Note that based on the FRF plot, the optimum actuator configuration for vibration control of the second mode should be concentrated in the vicinity of the corners of the examined area. This is consistent since maximum control authority for the second mode should be achieved by placing actuators 


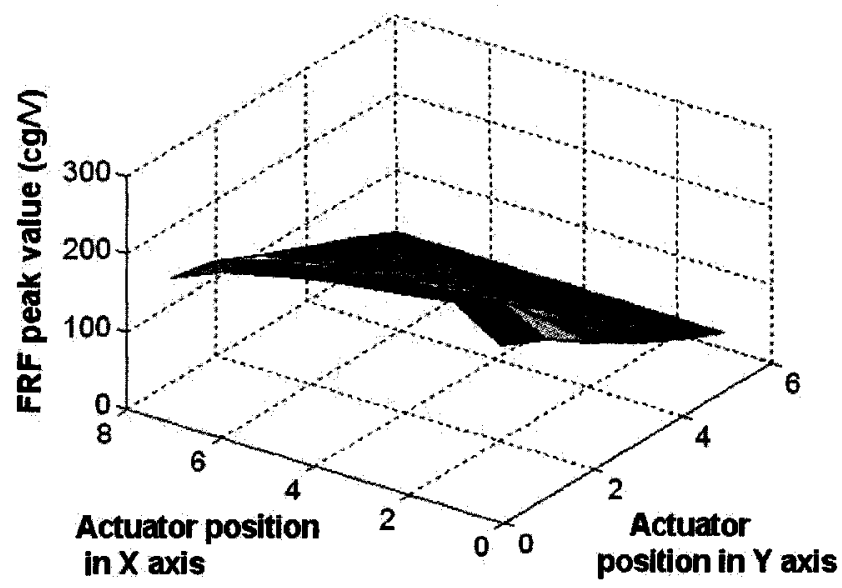

Figure 4.1: Discrete FRF Peak Magnitudes for the First Mode

as far as possible from the node line that runs through the center of the fin from root to tip.

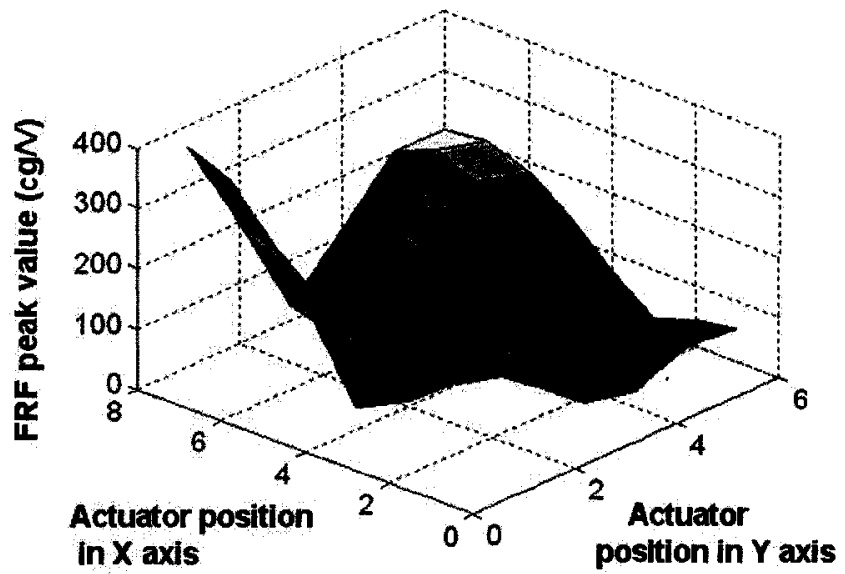

Figure 4.2: Discrete FRF Peak Magnitudes for the Second Mode

\subsubsection{Third Mode}

The third vibrational mode of the fin, or second bending mode, was excited at a frequency of approximately $73 \mathrm{~Hz}$. The peak FRF values at the reference point for the first mode are 
plotted vs. the centroid of actuator position in Figure 4.3. Note that for vibration control of the third mode, the optimum actuator position should be near the root of the fin or along the fifth row on the $\mathrm{Y}$ axis. This is consistent since maximum control authority for the third mode should be achieved by placing actuators as close as possible to the node lines that run along the fixed fin root and midway from root to tip. These both run from leading edge to trailing edge.

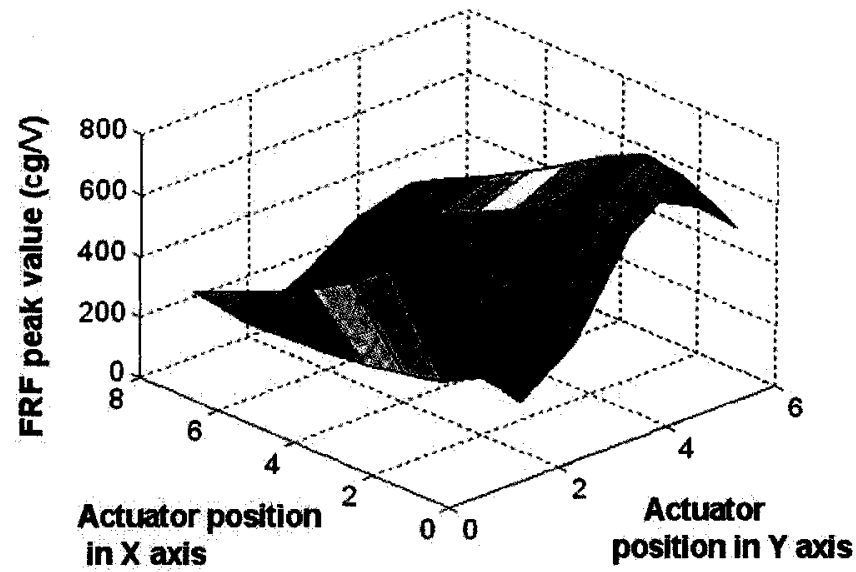

Figure 4.3: Discrete FRF Peak Magnitudes for the Third Mode

\subsection{Genetic Algorithm for Grouping Optimization}

As with the genetic algorithm for the single actuator pair problem, each individual was composed of a single chromosome containing a single gene. However, in the configuration optimization case, this gene was represented by a q-sized string of non-repeating integers ranging from one to forty-eight, where $\mathrm{q}$ was a pre-determined number of actuator pairs whose configuration was to be optimized. The resulting genetic algorithm was a hybrid, incorporating features of both binary and continuous genetic algorithms. Although like binary algorithms only a limited number of integer values were able to represent genes, the genes were not limited to $1 \mathrm{~s}$ or 0 s but instead to non-repeating integers representing the actuator positions. A binary algorithm was considered but had to be rejected due to 
difficulties that would occur with the reproduction process considering the requirement to maintain a fixed grouping size at each generation.

\subsubsection{Fitness Function}

The optimization objective was to maximize the actuation authority generated by actuator pairs at a combination of the 48 possible positions within the actuator test area of the fin shown in Figure 2.5. As defined in section 4.4.1, the peak FRF magnitudes obtained for actuators at these positions are direct measurements of actuation authority over acceleration (or if suitably scaled, displacement) recorded at the first accelerometer position shown in Figure 2.4. The fitness functions for individual modal and multi-modal control of acceleration and displacement were therefore derived directly from these peak FRF maginitudes.

Actuator configuration optimization for individual modes was a straightforward maximization of FRF peak magnitudes recorded for each individual mode. This in effect means that the actuator with the highest recorded FRF peak magnitude is the first selected, followed by the second and third, for as many actuators as were pre-specified for the configuration. Since the scaling of FRF results for displacement requires a division by the fundamental frequency for the relevant mode, the effect on the FRF from each possible actuator position is the same. There is therefore no distinction between acceleration and displacement control optimization for a single mode, and the fitness function for an actuator configuration is

$$
\text { Fitness }_{\text {Single mode }}=\sum_{i=1}^{q}(\text { Peak F RF Magnitudes }) \text {. }
$$

For multiple mode acceleration control, the FRF peak values could be used directly because an FRF is a normalized acceleration response. Hence the fitness function for a group was a weighted sum of the individual modal peak FRF values. Thus the fitness function for multi-modal acceleration control was 


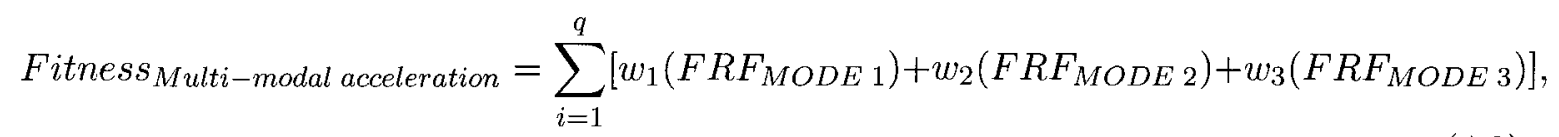

where $w_{1}, w_{2}$, and $w_{3}$ are the weighting factors on the first, second, and third modes, respectively.

Similarly for displacement control, considering harmonic motions, the fitness function was taken to be the sum of the weighted individual modal peak FRF values divided by the square of the fundamental frequencies for each mode. The objective was therefore to maximize

Fitness $_{\text {Multi-modal displacement }}=\sum_{i=1}^{q}\left[w_{1}\left(\frac{F R F_{M O D E 1}}{\omega_{1}^{2}}\right)+w_{2}\left(\frac{F R F_{M O D E 2}}{\omega_{2}^{2}}\right)+w_{3}\left(\frac{F R F_{M O D E 3}}{\omega_{3}^{2}}\right)\right]$.

For the actual optimization the weighting factors were taken as $w_{1}=w_{2}=w_{3}=1$; however, it is possible to use other weighting factors in order to achieve a specific emphasis on any of the three modes. For example, weighting factors of $w_{1}=1, w_{2}=0$, and $w_{3}=0.5$ would achieve bending control with a reduced emphasis on the third mode. The weighted summation of individual FRFs results in a simple fitness function that allows the contribution of individual modes to be modified according to the requirements of the problem.

An alternative fitness function using a square root of the sum of the squares of individual modal FRFs (as used for the polynomial optimization algorithm discussed in Chapter 3) was considered. This would have had the effect of increasing the importance of outlying modes. Although this was a desired result in the case of polynomial surface optimization, it was determined through numerical experimentation that increasing the contribution of an outlying mode was not beneficial in the optimization of group configuration, and that sufficient modal emphasis was provided through the use of user-defined weighting factors instead. 


\subsubsection{Genetic Algorithm Steps}

The genetic algorithm for multiple actuator pair configuration optimization was simpler than that for polynomial surface fitting, but more complex than that for single actuator pair position optimization. No initial population pool or range parameters were required since the alleles (actuator numbers) were constrained by the limits on possible actuator positions (1-48). Reproduction and mutation were relatively simple operations with the only major difference being the requirement for a forced mutation operation that is explained below. The computational requirements were very low, both in terms of generations to convergence (usually under 100), and computation time (several seconds). The algorithm terminated when there was no improvement in solution after 10 generations.

The genetic algorithm used for multiple actuator configuration optimization incorporates the following steps for a pre-selected number of actuators:

1. An initial population of ten individuals (trial sets of non-repeated actuators) is generated.

2. Reproduction is applied where each individual is bred with every other individual and also with itself (self-crossing). Breeding involves creating an offspring in which each allele (actuator position) has a $50 \%$ of chance of originating from each parent. If as a result of the transfer of a previous allele both parents would contribute a repeated allele, an immediate mutation is forced - "forced mutation" - on the child for the allele in question (see step 3). An example of where this could occur is shown in Table 4.1.

3. Each allele in each individual is subject to a small (typically $5 \%$ ) chance of mutation. Mutation simply results in a different non-repeated actuator position replacing the allele to be mutated. Forced mutation is the same process except that it has a $100 \%$ mutation probability.

4. The fitness of the bred and mutated individuals is calculated and the ten best individuals are selected as a new generation. 
5. Steps 2 to 4 are repeated until a specified number of generations have elapsed and the fittest set of actuator positions is identified.

Table 4.1: Example of a Forced Mutation (FM) Trigger During reproduction

\begin{tabular}{ccccccccccc}
\hline \hline First Parent & 34 & 22 & 18 & 43 & 23 & 9 & 29 & $\mathbf{2 5}$ & 40 & 5 \\
Second Parent & 25 & 38 & 24 & 16 & 2 & 7 & 42 & $\mathbf{1 8}$ & 35 & 22 \\
Child & $\mathbf{2 5}$ & 22 & $\mathbf{1 8}$ & 43 & 2 & 9 & 29 & FM & 40 & 5
\end{tabular}

\subsection{Assessing Configurations}

There are several important parameters that can be used to quantify the effectiveness of a grouping of actuators. Each gives different types of information about the optimization and is useful for assessing a particular condition.

\subsubsection{Actuation Authority}

The most basic measure of effectiveness is actuation authority. This is presented in terms of $\mathrm{g} / \mathrm{V}$ for acceleration, where $\mathrm{g}$ is the gravitational constant on the earth's surface $\left(9.8 \mathrm{~m} / \mathrm{s}^{2}\right)$ though often it is in centi-g or mili-g ( $\mathrm{cg}$ or $\mathrm{mg}$ ), and $\mathrm{V}$ is the voltage applied across the piezoelectric actuator in volts. For displacement, actuation authority is measured in terms of $\mathrm{mm} / \mathrm{V}$ for displacement. Although the total actuation authority of a group of actuators will always increase with added actuators, the incremental authority gained by adding each actuator always decreases as the number of actuators increases. A minimum number of actuators should be used in order to achieve vibration control for a particular loading condition. Once the required actuation authority is attained, additional actuators simply add undesired weight and power requirements. Actuation authority should therefore be used to determine the requisite number and configuration of actuators when a specific actuation authority is required to control a particular vibration condition. 


\subsubsection{Minimum Effectiveness (ME) and Relative Mean Effectiveness (RME)}

The efficiency of an actuator configuration can be represented by the parameters of minimum effectiveness (ME) and relative mean effectiveness (RME). Minimum effectiveness quantifies the effectiveness of the least effective actuator in the configuration relative to the most effective, i.e.,

$$
M E=\frac{\text { Actuation Authority of Actuator with Lowest Authority }}{\text { Actuation Authority of Actuator with Highest Authority }} .
$$

Relative mean effectiveness describes the mean actuation authority of all actuators in a configuration relative to the actuation authority of the most effective actuator, i.e.,

$$
R M E=\frac{\sum \text { Actuation Authorities } /(\text { No. of Actuators })}{\text { Actuation Authority of Actuator with Highest Authority }} .
$$

$\mathrm{ME}$ and RME are measures of the efficiency of an actuator configuration. Both parameters always decrease with actuators added to a set, but in some cases they decrease more rapidly with added actuators. For example, in the case of the second mode, although actuators bonded to the fin in the periphery of the actuator test area are effective for control of the second mode, actuators placed near the middle of the actuator test area have almost no effect. This can be seen in Figure 4.2. This is in contrast with the first mode, where an actuator pair bonded to the fin anywhere within the actuator test area will have a relatively large actuation authority. Therefore, optimization of actuator configuration is much more critical with the second mode than with the first. This is reflected in RME and ME, both of which decrease much more rapidly for the second mode as compared with the first. Note that ME and RME decrease much more rapidly for optimization of multi-modal control as opposed to control of a single mode, as will be seen in Tables 4.2 to 4.6 . This demonstrates that it is much more efficient to control a particular vibrational mode; inevitably, for a given number of actuators, the optimal configuration for a multi modal control will be less efficient than the optimal configuration for a single mode control. This is because for multi-modal control, the configuration is a trade-off between various sub-configurations, 
each of which is optimized for a particular mode.

\subsection{Optimization Types}

The results presented below can be used to either optimize the configuration of actuators when due to some restriction such as weight, the objective is to use a particular predetermined number of actuators, or to optimize the configuration as well as the number of actuators simultaneously in order to achieve a required actuation authority, depending on design criteria. All types of multi-modal optimizations can have weighting factors applied to the fitness function in order to emphasize relative modal contribution.

\subsubsection{Actuation Maximization}

Actuation maximization is used to maximize the actuation authority generated at a reference point (in this case at accelerometer 1 in Figure 2.4) by a pre-determined number of actuator pairs. Any number of actuators can be used, but generally less than about ten actuator pairs are employed. Actuation maximization is also a sub-process and starting point for both the minimization of the number of actuators and trade-off studies. This is because the actuation authority should always be maximized for a specific number of actuators, regardless of any other criteria. Actuation maximization is achieved by determining which of a specific number of actuator pairs out of the 48 possible should be activated to meet an actuation requirement. This requirement can be maximization of authority for individual modes, or multi-modal acceleration or displacement. Actuation maximization is critical; as will be shown later with the second mode, it can result in almost three times the mean actuation authority achieved by random actuator placement, and over ten times the actuator authority generated by the worst possible actuator placement. 


\subsubsection{Minimization of Number of Actuators}

This type of optimization is applicable when a specific magnitude of actuation authority is required for a problem-specific task. This can be for an individual mode, or for multimodal acceleration or displacement control. This is actually an application of actuation maximization, where instead of optimizing activation of a pre-determined number of actuators the minimum number of actuators is determined for a pre-specified required actuation authority.

\subsubsection{Trade-off Study}

A trade off study is used to examine the relationship between the increasing actuation authority and the increasing voltage requirement or added mass with each additional actuator. The simplest type of trade-off study is a plot of actuation authority (a non-linear quantity) together with actuator mass or voltage requirement (in this case a linear quantity) versus the number of actuators. This allows a direct comparison of the trend between the benefit of adding actuators in terms of added actuation authority and the cost of the added weight or voltage requirements. In order to actually determine an optimum actuator configuration using a trade-off study, a problem specific cost-benefit relationship is required. This relationship can then be used to determine the optimum actuator configuration, either graphically from a plot of benefits and costs, or using an algorithm such as one of the genetic algorithms presented in this thesis. If a genetic algorithm is used, the cost-benefit relationship is incorporated directly into the fitness function. It is possible for the cost associated with increasing voltage to increase non-linearly. For example, a fitness function reflecting the fact that power requirements are proportional to the square root of the generated voltage (assuming a constant resistance and DC) could be employed. An example of a trade-off study configuration optimization is presented in Section 4.7. 


\subsection{Configurations for Actuation Maximization}

Actuation optimization was performed for many predetermined numbers of actuators for each of the first three modes individually, and for multi-modal acceleration and displacement control. In the case of multi-modal control, the weighting factors were all taken to be 1 , meaning that no mode was particularly emphasized. The actuation maximization results are outlined below. Figure 4.4 shows the numbering scheme used for all possible actuators.

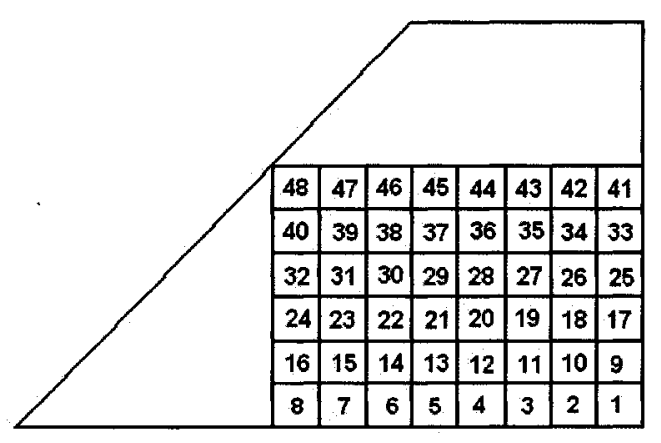

Figure 4.4: Actuator Numbering Scheme

\subsubsection{First Mode}

Actuation maximization configurations for the first mode are shown with $1,2,5$, and 10 actuator pairs in Table 4.2. The actuation maximization configurations for 5 and 10 actuators are plotted in Figure 4.5. The configurations agree with the FRF peak magnitude results shown in Figure 4.1 and the actuators are positioned along the fin root as close as possible to the first bending node line. The RME and ME decreases relatively slowly for the first mode, with a RME of $86 \%$ and a ME of $77 \%$ for 10 actuators. The ME of the twoactuator configuration was $97 \%$, indicating that the second actuator selected was almost as effective as the first. The drop in actuator effectiveness is relatively slow for the first mode, indicating that while actuation maximization is important, it is less critical for the first mode. A random scattering of actuators within the actuator test area would still be 
able to maintain a moderate level of vibration control over the first mode.

Table 4.2: Selected Actuation Maximization Configurations for the First Mode

\begin{tabular}{ccccc}
\hline \hline No. Actuators & Activated Actuators & Actuation Authority $(\mu \mathbf{g} / V)$ & $\mathrm{ME}$ & $\mathrm{RME}$ \\
1 & 2 & 2.1 & 1 & 1 \\
2 & 2,3 & 4.2 & 0.97 & 0.98 \\
5 & $2-5,10$ & 9.8 & 0.85 & 0.92 \\
10 & $1-7,10-12$ & 18 & 0.77 & 0.86
\end{tabular}

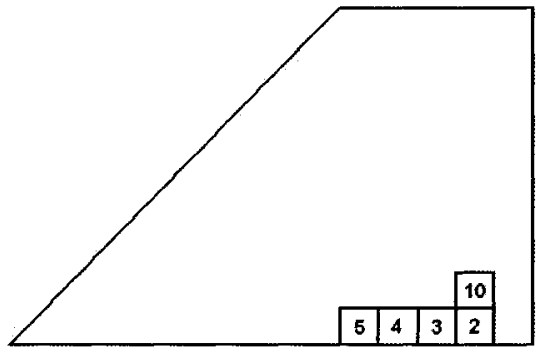

(a) 5 Activated Actuators

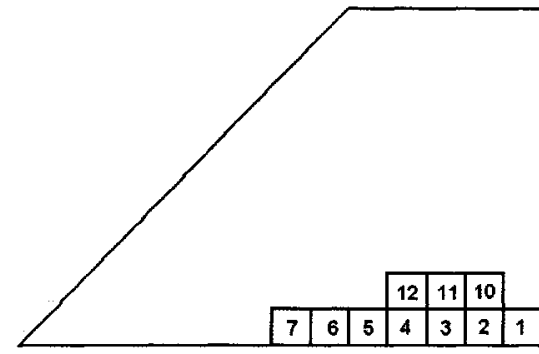

(b) 10 Activated Actuators

Figure 4.5: Actuation Maximization Configurations for the First Mode

\subsubsection{Second Mode}

Actuation maximization configurations for the second mode are shown with $1,2,5$, and 10 actuator pairs in Table 4.3. The effectiveness of actuation maximization is evident from a comparison with the worst possible configuration of 10 actuators (the 10 actuators with the lowest control authority as determined by using an inverse fitness function), and the mean configuration of 10 actuators (the average control authority if actuators were randomly placed). The mean configuration does not correspond to an actual actuator configuration, because instead of representing an actual selection of actuators, it assumes that all actuators contribute exactly the mean actuation authority of all 48 possible actuators. The actuation maximization configurations for 5 and 10 actuators are plotted in Figure 4.6. The configurations agree with the FRF peak magnitude results shown in Figure 4.2. As expected, the 
actuators are positioned as far as possible from the first torsional node line.

Table 4.3: Selected Actuation Maximization Configurations for the Second Mode

\begin{tabular}{ccccc}
\hline \hline No. Actuators & Activated Actuators & Actuation Authority $(\mu \mathrm{g} / V)$ & $\mathrm{ME}$ & $\mathrm{RME}$ \\
1 & 8 & 3.8 & 1 & 1 \\
2 & 1,8 & 7.4 & 0.86 & 0.93 \\
5 & $1,2,6-8$ & 17 & 0.69 & 0.88 \\
10 & $1-2,6-8,38-40,47-48$ & 27 & 0.57 & 0.72 \\
10 -mean configuration & $\mathrm{N} / \mathrm{A}$ & 11 & $\mathrm{~N} / \mathrm{A}$ & 0.31 \\
10-worst configuration & $12,21-27,35,43$ & 1.9 & 0.01 & 0.05
\end{tabular}

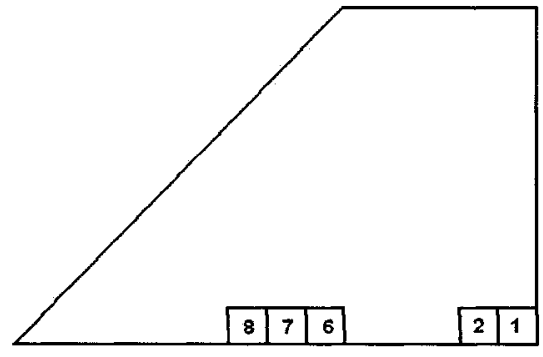

(a) 5 Activated Actuators

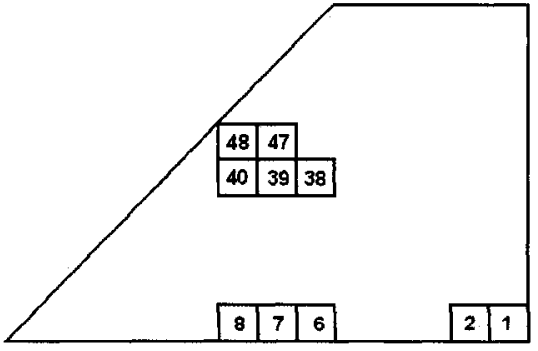

(b) 10 Activated Actuators

Figure 4.6: Actuation Maximization Configurations for the Second Mode

The RME decreases relatively rapidly for the second mode, with a RME of $72 \%$ for 10 actuators. Also of note is the ME, which indicates that the second actuator selected was only $86 \%$ as effective as the first, and that the fifth actuator selected was only $69 \%$ as effective as the first. Therefore, it is much more efficient to use a smaller number of actuators if sufficient actuation authority could still be achieved. This number of actuators could be determined through an actuator minimization optimization. The mean and worst configurations for 10 actuators are of particular note. For a set of 10 actuators, the mean configuration had a RME of only $31 \%$, as compared with $72 \%$ for the optimized configuration. This indicates that a configuration obtained by randomly choosing groupings of actuators on average would be only $40 \%$ as effective as the optimized configuration. The worst possible configuration 
of 10 actuators had a RME of only $5 \%$, and the worst actuator had only $1 \%$ of the control authority of the best possible actuator. On average, less control authority would be achieved by randomly placing 10 actuators than by using an actuation maximization to determine the position of 5 .

\subsubsection{Third Mode}

Actuation maximization configurations for the third mode are shown with 1, 2, 5, and 10 actuator pairs in Table 4.4. The actuation maximization configurations for 5 and 10 actuators are plotted in Figure 4.7. The configurations agree with the FRF peak magnitude results shown in Figure 4.3. The actuators are positioned along the fin root as close as possible to the first bending node line, or alternatively clustered around the second bending node line running from leading to trailing edge roughly midway from the fin root to tip. The RME and ME decreases moderately for the third mode, with a RME of $83 \%$ and a ME of $72 \%$ for 10 actuators. The ME of the two-actuator configuration was $93 \%$, indicating that the second actuator selected was still very effective, but not nearly as much as the second most effective actuator for the first mode. The drop in actuator effectiveness is moderate for the third mode, indicating that actuation maximization is more important than for the first, but less than for the second. A random scattering of actuators within the actuator test area would be unlikely to be able to maintain an acceptable level of vibration control over the third mode.

Table 4.4: Selected Actuation Maximization Configurations for the Third Mode

\begin{tabular}{ccccc}
\hline \hline No. Actuators & Activated Actuators & Actuation Authority $(\mu \mathrm{g} / V)$ & $\mathrm{ME}$ & $\mathrm{RME}$ \\
1 & 2 & 7.7 & 1 & 1 \\
2 & 2,34 & 15 & 0.93 & 0.97 \\
5 & $1,233-35$ & 35 & 0.85 & 0.91 \\
10 & $1-3,25-27,33-36$ & 64 & 0.72 & 0.83
\end{tabular}




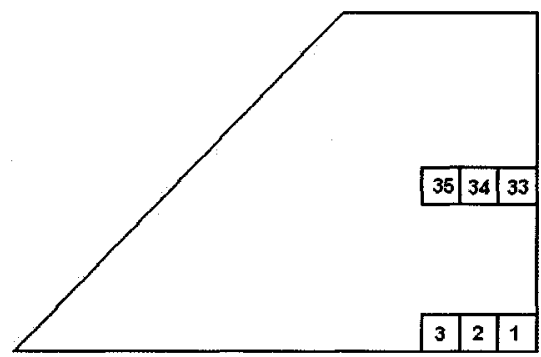

(a) 5 Activated Actuators

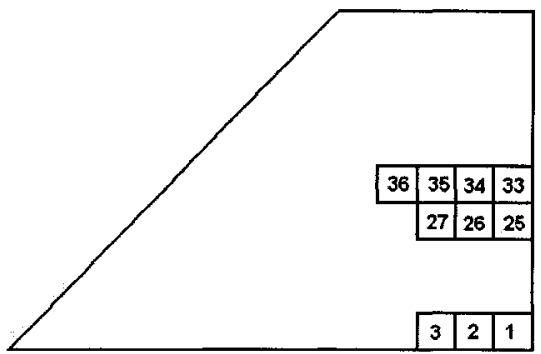

(b) 10 Activated Actuators

Figure 4.7: Actuation Maximization Configurations for the Third Mode

\subsubsection{Multi-Modal Acceleration}

Actuation maximization configurations for multi-modal acceleration control are shown with selected numbers of actuator pairs in Table 4.5. A mean configuration of 10 randomly selected actuators is also shown for comparison. The actuation maximization configurations for 2, 5, 10, and 24 actuators are plotted in Figures 4.9 and 4.10. The ME and RME for up to 24 actuators are plotted in Figure 4.8. The actuation maximization configurations for acceleration control maximized the combined acceleration control authority from the first three modes with equal weighting on each mode. The positioning of actuators was thus a compromise between control authority for the first three modes. Although these configurations are not ideally suited to control any mode individually, they provide the best overall actuation authority for acceleration control of the modes.

The RME for acceleration control decreased rapidly, to a RME of $64 \%$ for 10 actuators. The ME dropped markedly after the second actuator; the third actuator chosen was only $68 \%$ as effective as the first. The rapid decrease in ME and RME resulted from the compromise between modal actuation authority. The multi-modal fitness function inevitably leads to a reduced overall actuation efficiency, but allows more flexibility of control over individual modes. This demonstrates the importance of understanding the anticipated loading conditions for a structure. A superior design is achieved by designing an active structure to control as narrow a band of excitation as possible. However, if the in-service loading 
conditions are not fully understood, it is often necessary to incorporate a more flexible design. This flexibility is achieved at the cost of efficiency. A higher efficiency could also be achieved by selecting fewer actuators (at the cost of actuation authority) or applying different weighting factors in order to place more emphasis on a particular mode (at the cost of flexibility).

Table 4.5: Selected Actuation Maximization Configurations for the Multi-modal Acceleration Control

\begin{tabular}{ccccc}
\hline \hline No. Actuators & Activated Actuators & Actuation Authority $(\mu \mathrm{g} / V)$ & $\mathrm{ME}$ & $\mathrm{RME}$ \\
1 & 2 & 14 & 1 & 1 \\
2 & 1,2 & 25 & 0.91 & 0.92 \\
3 & $1-3$ & 34 & 0.68 & 0.83 \\
5 & $1-3,33,34$ & 50 & 0.64 & 0.73 \\
8 & $1-3,6,7,33-35$ & 73 & 0.59 & 0.67 \\
10 & $1-3,5-8,33-35$ & 87 & 0.56 & 0.64 \\
12 & $1-3,5-8,26,33-36$ & 100 & 0.55 & 0.62 \\
16 & $1-8,25,26,33-38$ & 130 & 0.54 & 0.59 \\
20 & $1-8,25-29,33-39$ & 160 & 0.48 & 0.57 \\
24 & $1-8,25-30,33-42$ & 180 & 0.43 & 0.54 \\
$10-$ mean configuration & N/A & 57 & N/A & 0.40
\end{tabular}

\subsubsection{Multi-Modal Displacement}

Actuation maximization configurations for multi-modal displacement control are shown with selected numbers of actuator pairs in Table 4.6. A mean configuration of 10 randomly selected actuators is also shown for comparison. The actuation maximization configurations for 2, 5, 10, and 24 actuators are plotted in Figures 4.12 and 4.13. The ME and RME for up to 24 actuators are plotted in Figure 4.11. The actuation maximization configurations for displacement control maximized the combined displacement control authority from the first three modes with equal weighting on each mode. The positioning of actuators was thus a compromise between control authority for the first three modes. Although these 


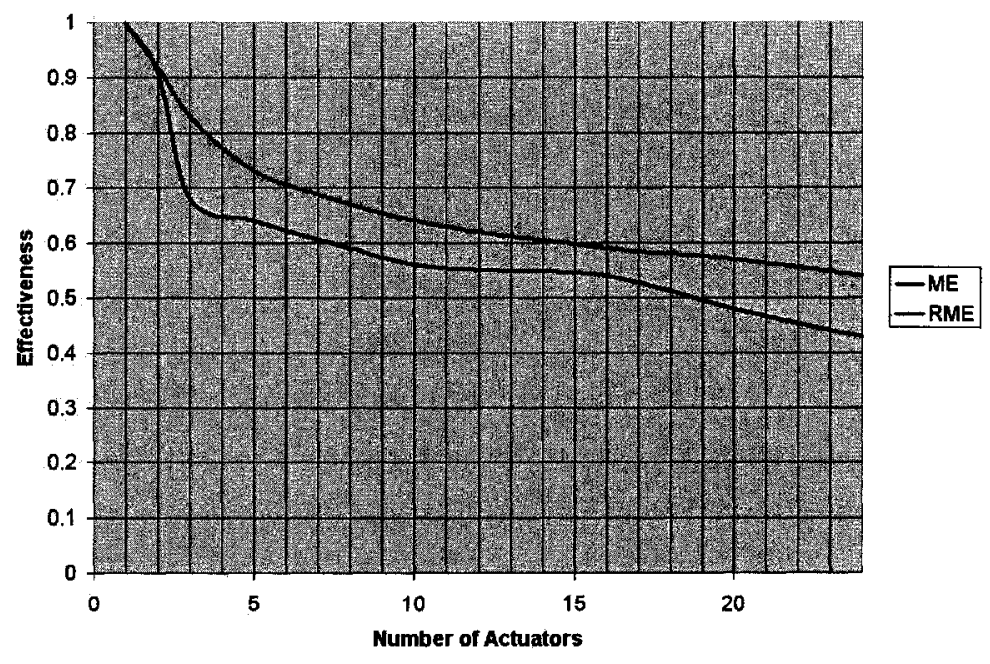

Figure 4.8: Minimum Effectiveness (ME) and Relative Mean Effectiveness (RME) versus Number of Actuators for Acceleration Control

configurations are not ideally suited to control any mode individually, they provide the best overall actuation authority for displacement control of the modes. Unlike with an individual mode, configurations for displacement control are significantly different from configuration used for acceleration control. This is caused by relative modal contribution in acceleration and displacement. The same effect could be achieved by using appropriate weighting factors.

The RME for displacement control decreased more slowly than for acceleration control, to a RME of $75 \%$ for 10 actuators. The ME also decreased significantly more slowly, with the tenth actuator $64 \%$ as effective as the first. This indicates that although there is a significant compromise between individual modes for acceleration control, the multi-modal configurations for displacement control are more efficient than for acceleration control as a result of greater dominance by the lower two modes. As a result, there is less of a trade-off between flexibility and efficiency. However, as with acceleration, the multi-modal fitness function does lead to a reduced overall actuation efficiency. The RME and ME decrease more rapidly for multi-modal displacement control than for the first or third mode individually, but less rapidly than for the second. As the number of actuators increased, the efficiency advantage of multi-modal displacement relative to acceleration dissipated. For 24 actuators the RME was $59 \%$ for displacement control as compared to $54 \%$ for acceleration 


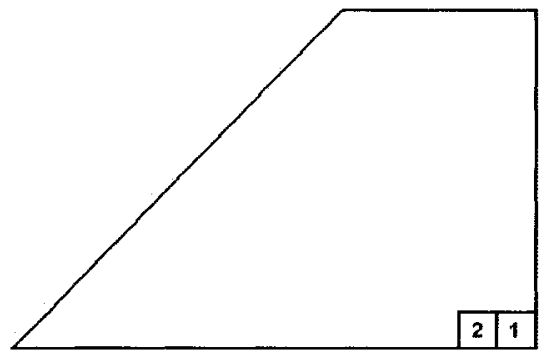

(a) 2 Activated Actuators

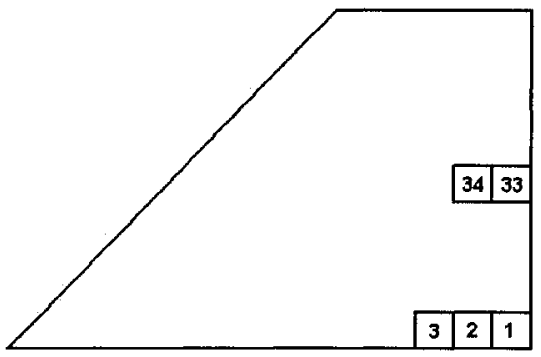

(b) 5 Activated Actuators

Figure 4.9: Actuation Maximization Configurations for Multi-modal Acceleration Control

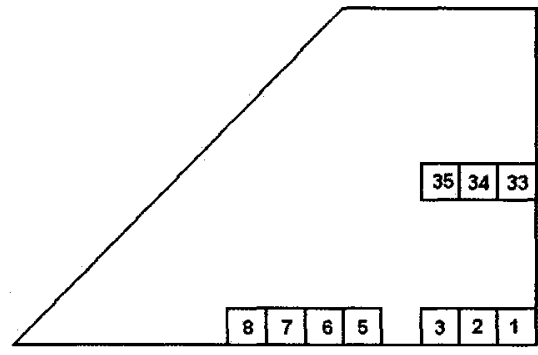

(a) 10 Activated Actuators

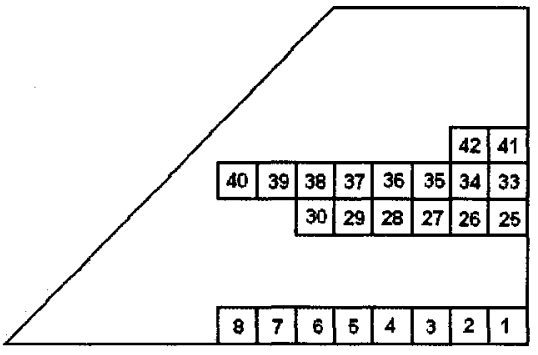

(b) 24 Activated Actuators

Figure 4.10: Actuation Maximization Configurations for Multi-modal Acceleration Control

control.

The dominance of the first mode resulted from the much lower first fundamental frequency as compared with the higher modes and agrees with theoretical and experimental results. As a result, the actuation maximization configurations for displacement control were similar to those for first modal control as shown in Table 4.2. However, the differences between the optimal configurations demonstrate some contribution from the second and third modes. As with the second mode and acceleration cases, the optimized configuration for 10 actuators generated higher actuation authority than a mean configuration. However, this was somewhat less significant for displacement control than for acceleration control. 
Table 4.6: Selected Actuation Maximization Configurations for the Multi-modal Displacement Control

\begin{tabular}{ccccc}
\hline \hline No. Actuators & Activated Actuators & Actuation Authority $(m m / V)$ & ME & RME \\
1 & 2 & 0.77 & 1 & 1 \\
2 & 1,2 & 1.4 & 0.86 & 0.92 \\
3 & $1-3$ & 2.0 & 0.85 & 0.88 \\
5 & $1-6$ & 3.2 & 0.77 & 0.83 \\
8 & $1-7,10$ & 4.9 & 0.72 & 0.78 \\
10 & $1-8,10,11$ & 5.8 & 0.64 & 0.75 \\
12 & $1-12$ & 6.7 & 0.59 & 0.72 \\
16 & $1-16$ & 8.4 & 0.51 & 0.67 \\
20 & $1-20$ & 9.8 & 0.47 & 0.63 \\
24 & $1-22,26,28$ & 11 & 0.39 & 0.59 \\
$10-$ mean configuration & N/A & 4.2 & N/A & 0.54
\end{tabular}

\subsection{Example of a Trade-off Study}

This trade-off study is a theoretical example of how a benefit vs. cost approach could be used to optimize the configuration and number of actuators on the flexible fin.

Assuming the task is to minimize vibration magnitude as measured at accelerometer 1, the benefits associated with additional actuators is increasing displacement actuation authority in $\mathrm{mm} / \mathrm{V}$. If the anticipated loading conditions are low frequency excitations in the range of $1-50 \mathrm{~Hz}$, we can consider only the first two modes (fundamental frequencies of $17.5 \mathrm{~Hz}$ and $47 \mathrm{~Hz}$ ). Since there is a much larger loading range affecting the first mode, let us take $\mathrm{w}_{1}=1$ and $\mathrm{w}_{2}=0.5$.

There are two costs associated with using additional actuators: power requirements and weight. Assuming the multiple actuator results can be used only for a selected group of actuators, the cost associated with weight is linear. In reality there would be no passive actuators on the fin, so there would also be a variable stiffness to consider. Assuming DC electricity and constant resistance, the power required can be expressed as 


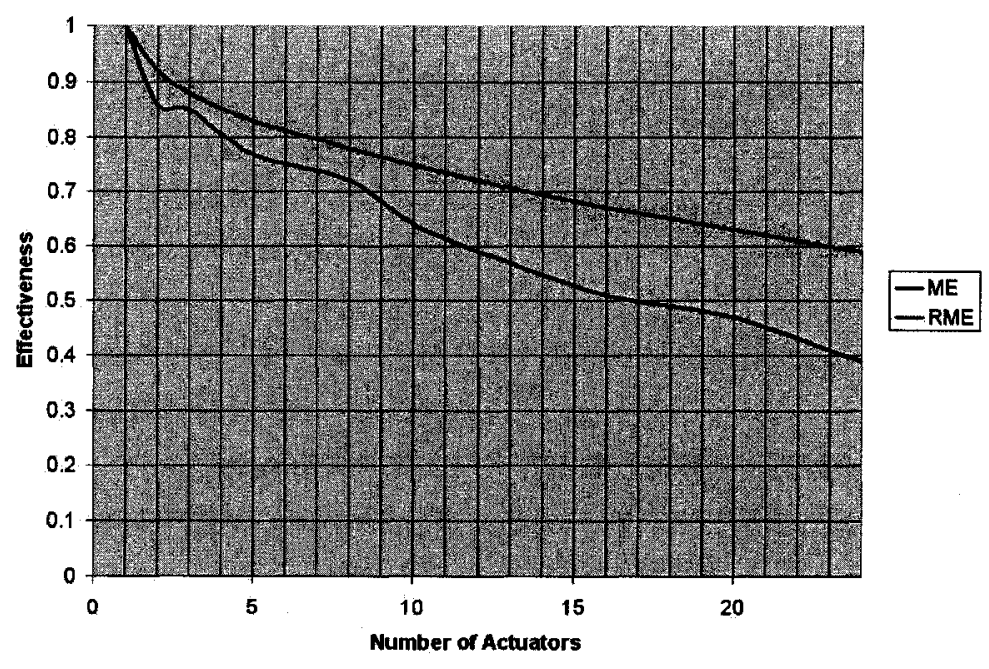

Figure 4.11: Minimum Effectiveness (ME) and Relative Mean Effectiveness (RME) versus Number of Actuators for Displacement Control

$$
\text { Power Required }=\left(V^{2}\right) / R \text {, }
$$

where $\mathrm{V}$ is the voltage and $\mathrm{R}$ is the resistance. Assuming that maximum voltage is delivered to all actuators, the required voltage is proportional to the number of actuators and thus

$$
\text { Number of Actuators } \propto \sqrt{\text { Power Required }}, \text { or }
$$

$$
\text { Power Required } \propto(\text { No.Actuators })^{2} \text {. }
$$

Assuming power requirements and weight are scaled so that they contribute equally to the cost, we get

$$
\text { Costs } \propto \text { No.Actuators }+(\text { No.Actuators })^{2} .
$$

The optimal configuration depends on the relationship that is set between costs and benefits. A simple method of scaling the problem would be to specify that the relationship 


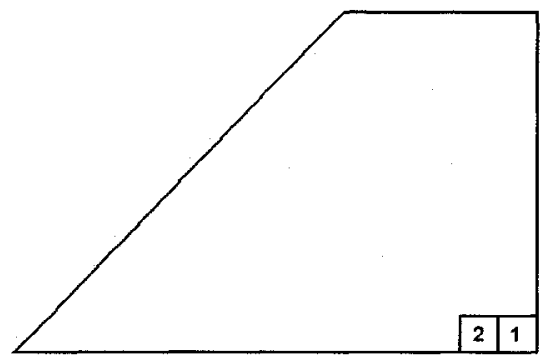

(a) 2 Activated Actuators

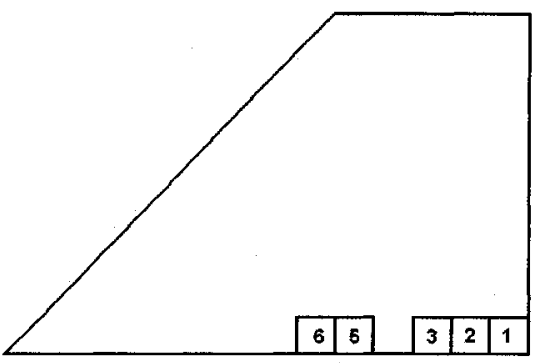

(b) 5 Activated Actuators

Figure 4.12: Actuation Maximization Configurations for Multi-modal Displacement Control

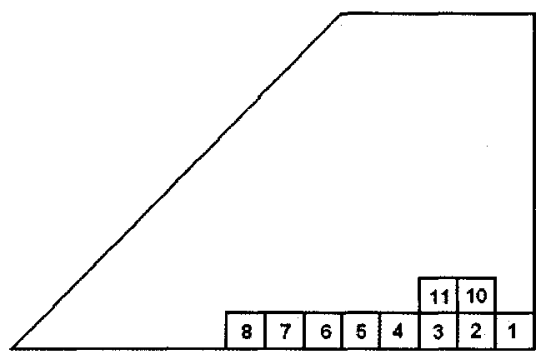

(a) 10 Activated Actuators

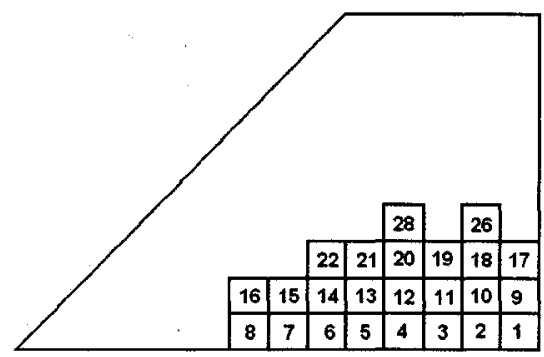

(b) 24 Activated Actuators

Figure 4.13: Actuation Maximization Configurations for Multi-modal Displacement Control

between costs and benefits converge with a single actuator. The objective then is to plot costs and benefits on the same plot and determine the point of maximum separation. 


\section{Chapter 5}

\section{Verification of Simulation through}

\section{Experimentation}

This chapter describes the verification of the simulation results through comparison to experiment. Simulation results from a finite element model of the flexible fin are compared with experimental results obtained from an apparatus in an identical configuration. The experimental configuration is shown in Figure 2.2 and the finite element model is shown in Figure 2.3. A comparison of the natural frequencies for the FEM and experimental models is shown in Figure 5.1. Actuation maximization is applied to both models of the fin and the resulting actuator configurations are compared. An actuator equivalence is also developed so that a comparison of the experimental results can be made with the results obtained in Chapters 3 and 4 .

Table 5.1: Comparison of Natural Frequencies from Simulation and Experimental Model

\begin{tabular}{cccc}
\hline \hline Mode & Exp. Nat. Freq. (Hz) & FEM Nat. Freq. (Hz) & Difference (\%) \\
1 & 17.9 & 17.0 & -5.3 \\
2 & 53.8 & 49.1 & -8.7 \\
3 & 83.5 & 80.4 & -3.7 \\
4 & 126.0 & 121.1 & -3.9 \\
5 & 172.0 & 166.4 & -3.3
\end{tabular}




\subsection{Experimental Data Acquisition}

Figure 5.1 shows the flexible fin model with attached actuator pairs while Figure 5.2 shows the experimental test bed developed at the SDSMP laboratory of the NRC of Canada. The FRF was recorded for the experimental model of the fin for the activation of each of the actuator pairs. $\mathrm{A}+/-50$ volt $(100 \mathrm{Vpp})$ bidirectional chirp signal that swept the frequency range of $1-100 \mathrm{~Hz}$ was used to excite each actuator pair in turn, while the FRF was recorded at all accelerometers in real time using Real-Time Workshop and xPC TargetBox of MathWorks. The sampling rate was $1000 \mathrm{~Hz}$ for a total of 4 minutes (2 minutes in each direction). For each accelerometer, the FRFs were recorded in an array associated with each actuator. The sampling rate and time meant that the arrays were composed of 240,000 entries (4 minutes at 1000 cycles per second).

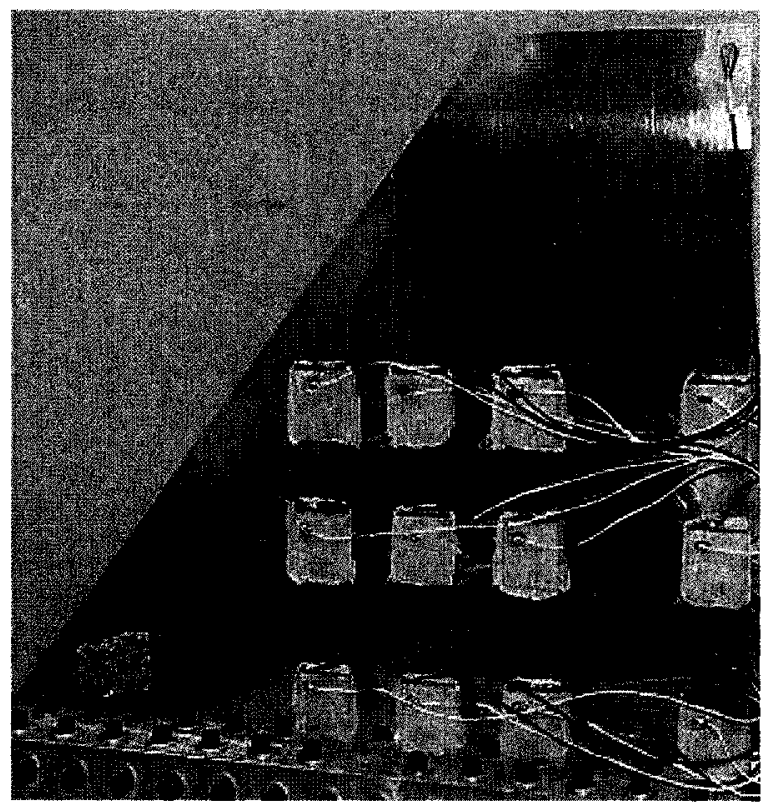

Figure 5.1: Flexible Fin with Piezoelectric Actuators [3]

\subsubsection{Test Bed Specifications}

The flexible fin test bed used for the purposes of this study was developed at the ASDSMP laboratory of the National Research Council of Canada. It consisted of a flexible aluminum 


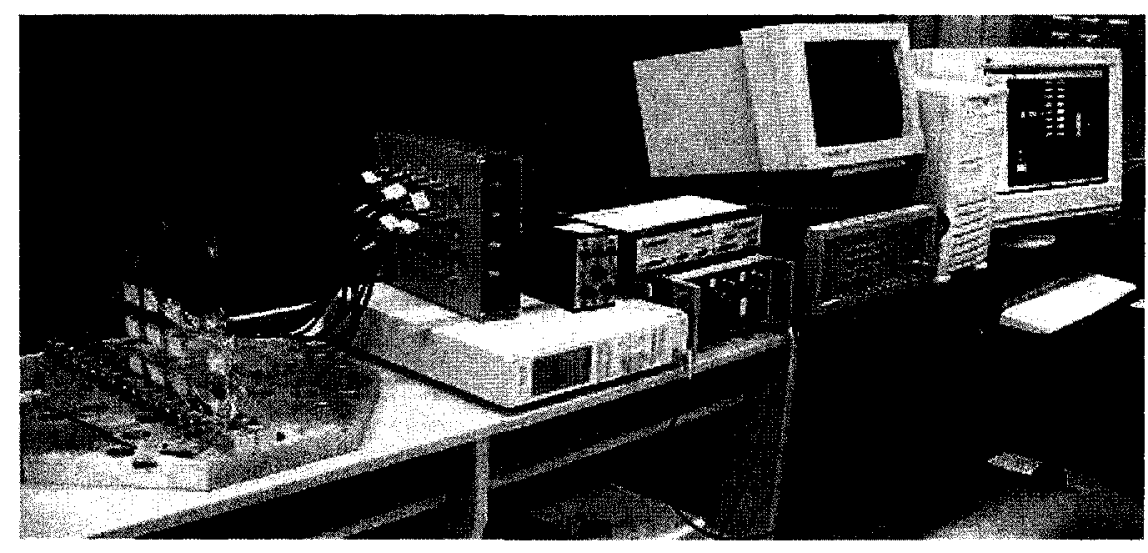

Figure 5.2: The Experimental Test Bed [3]

fin of $1 \mathrm{~mm}$ thickness cantilevered at the fin root between a pair of solid aluminum blocks. Twelve lead zirconium titanate (PZT) thin ceramic plate actuators were bonded to each side of the fin as shown in Figure 5.1 [4]. The material properties of the fin aluminum (2024-T3) and actuator piezoelectric material (BM500 from SensorTech Systems, Inc.) are shown in Table 5.2. The accelerometer positions, serial numbers, and sensitivities for the flexible fin test bed are shown in Table 5.3. These accelerometers are equivalent to those listed in Table 2.1 for the finite element model.

Table 5.2: Material Properties of Aluminum and Piezoelectric Material

\begin{tabular}{ccc}
\hline Property & Aluminum 2024-T3 & PZT BM500 \\
\hline \hline Density $\left(\mathrm{Kg} / \mathrm{m}^{3}\right)$ & 2796 & 7650 \\
Elastic Modulus $(\mathrm{MPa})$ & 73.0 & 64.58 \\
Thermal Expansion $\left(\mu \mathrm{m} \cdot{ }^{\circ} \mathrm{C}\right)$ & 23.2 & - \\
Thickness $(\mathrm{mm})$ & 1.02 & 0.50 \\
Charge Constant, $\mathrm{d}_{31}(\mathrm{pC} / \mathrm{N})$ & - & 175 \\
\hline
\end{tabular}

\subsubsection{Experimental Results}

Matlab was used to extract the experimental FRF peak magnitudes that were stored in arrays associated with each accelerometer for each activated actuator. These FRFs were 
Table 5.3: Accelerometer Information for the Flexible Fin Test Bed

\begin{tabular}{cccc}
\hline Accel & $\mathrm{X}(\mathrm{mm})$ & $\mathrm{Y}(\mathrm{mm})$ & Serial No. \\
\hline \hline 1 & 8.8 & 245.4 & 11474 \\
2 & 181.7 & 178.2 & 8700 \\
3 & 114.8 & 245.4 & 8704 \\
4 & 10.0 & 168.2 & 8701 \\
5 & 8.8 & 210.2 & 8699 \\
\hline
\end{tabular}

plotted versus excitation frequency in order to determine the peak FRF magnitude associated with each of the three first modes. A typical example is shown in Figure 5.3. The peak FRF magnitudes for the activation of each individual actuator were then selected and tabulated. These are presented in Tables 5.4 to 5.6.

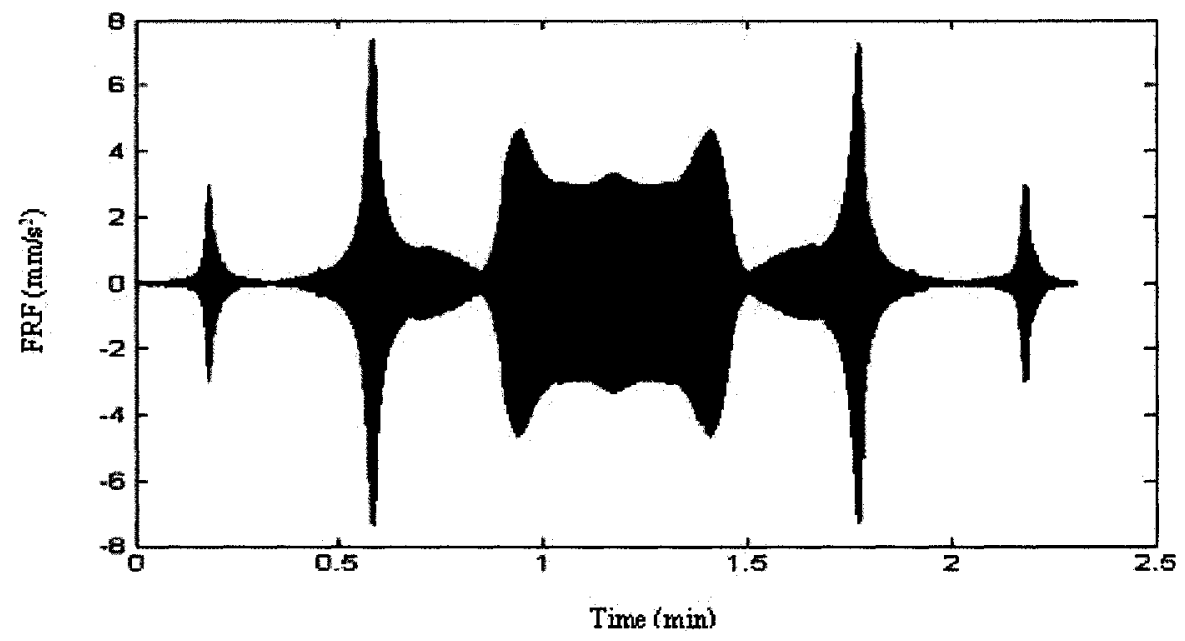

Figure 5.3: A Typical FRF vs. Time Plot

\subsubsection{Scaling of Experimental Results}

A $3.4 \%$ structural damping ratio was used for the finite element simulation in Patran. This value was adopted in order to obtain results that were consistent with those obtained experimentally for the first mode. This resulted in simulation values being poorly scaled in comparison with those obtained from the experimental model of the fin for the second and 
third mode. Scaling factors of 0.71 and 0.53 were applied to the simulation results obtained for the second and third mode, respectively. These factors had the effect of equalizing the mean peak FRF magnitudes between the respective experimental and simulation results. This scaling could easily have been alternatively introduced as weighting factors of 1.4 $(1 / 0.71)$ and $1.9(1 / 0.53)$ on the second and third mode, respectively. In effect, using the peak magnitudes of the second and third modes from the simulation results would be equivalent to assigning higher weighting factors to these modes in multi-modal vibration control. In individual modal control this has no effect, since only the relationships between FRFs are relevant.

\subsection{Comparison of Experimental Results with Simulation}

Although there was initially poor scaling between the results for the second and third modes, there was very good agreement between simulation and experiment for relative contributions of the actuators. There was, therefore, good agreement between the optimized configurations obtained by simulation and experiment. The minor differences noted were probably due to modelling assumptions such as damping, ignoring adhesive layers, etc. The experimental results were compared with the simulation results for three configurations of the finite element model: an FEM model of the experimental configuration, the multiple actuator optimization model from Chapter 4, and the single actuator optimization model from Chapter 3. These comparisons are described below.

\subsubsection{Comparison with Results from FEM model in Experimental Con- figuration (12 Actuators)}

Comparisons of the experimental results with the scaled simulation results for the first, second, and third modes are shown in Tables 5.4 to 5.6. The experimental results are in quite close agreement with the simulation results, with mean differences of $-0.6 \%,-4.6 \%$, and $4.8 \%$, for the first, second, and third modes, respectively. When the absolute difference in actuation authority magnitude is considered, the results are in reasonable, though reduced, 
agreement, particularly for the second and third modes. However, the optimum actuators for individual mode or multi-modal vibration control is of much more relevance than the actuation authority. It is useful to rank the actuators in order of their effectiveness for each type of control. A direct comparison of the optimum configurations for any pre-determined number of actuators can then be made between the experimental and simulation results. Tables 5.7 and 5.8 show these comparisons for individual and multi-modal acceleration and displacement control, respectively. These are rearrangements of the results shown in Tables 5.4 to 5.6, ranking the actuators in terms of the order of effectiveness from most effective to least in terms of the fitness function associated with the type of control. The numbers listed in the Tables are the numbers associated with the actuators from Figure 2.3. The tables can be used to determine an optimal configuration for any pre-determined number of actuators by using the actuators that are more effective than the actuator with a ranking equal to the number desired. The tables demonstrate the good agreement between experimental and simulation results, with the results generally being consistent or differing by a single rank.

\subsubsection{Comparison with Results from Multiple Actuator Configuration Optimization (Chapter 4)}

It is also possible to compare the experimental results with the simulation results obtained from the fin configuration used in the multiple actuator optimization problem (48 actuators). The configurations are similar since one actuator is activated at a time from a distributed set, and the geometry of the fin and actuators are the same. The only difference is the number of passive actuators. Since there are 47 passive actuators for the multiple actuator activation

problem and only 11 for the experimental model, the FRF peak magnitudes should be quite different, but the rankings of optimum actuator positions should be fairly similar. In order to compare the results obtained by using the FEM model with 48 actuators to the experimental results, the actuators from the multiple actuator optimization problem must be expressed as the nearest equivalent actuators from the experimental configuration. These represent the closest translation of each of the 48 actuators to one of the 12 actuators in the reduced order model. When equivalent actuators are used for the first 5 experimental actuators, as shown 
Table 5.4: Comparison of Experimental and Simulation Results for the First Mode of the Smart Fin

\begin{tabular}{cccc}
\hline Actuator & Exp. Peak FRF $\left(\mathrm{mm} / \mathrm{s}^{2}\right)$ & FEM Peak FRF $\left(\mathrm{mm} / \mathrm{s}^{2}\right)$ & Difference $(\%)$ \\
\hline \hline 1 & 8.14 & 8.99 & 10 \\
2 & 8.73 & 9.69 & 11 \\
3 & 8.91 & 9.17 & 3 \\
4 & 7.35 & 7.76 & 5 \\
5 & 4.80 & 5.11 & 5 \\
6 & 5.97 & 5.64 & -7 \\
7 & 5.89 & 5.29 & -11 \\
8 & 5.13 & 4.76 & -8 \\
9 & 2.61 & 2.64 & 1 \\
10 & 3.26 & 3.00 & -9 \\
11 & 3.04 & 3.00 & -2 \\
12 & 2.97 & 2.82 & -6 \\
Mean abs. diff. & - & - & 6.8 \\
Mean diff. & - & - & -0.6 \\
\hline
\end{tabular}

in Tables 5.9 and 5.10 , it is possible to verify the results of the multiple actuator activation problem using the experimental model. The results are quite consistent, with rankings generally being the same or one rank removed. The minor differences probably arose due to the lack of modal scaling that was applied to the simulation results in the previous section, the difference in the number of passive actuators, and modelling assumptions and deficiencies as discussed in Chapter 2.

\subsubsection{Comparison with Results from Single Actuator Positional Opti- mization (Chapter 3)}

The results obtained through simulation for the single actuator optimization problem using the finite element model of the fin also agreed well with the results obtained through experiment using the smart fin test bed if allowances are made for the differences in configuration. 
Table 5.5: Comparison of Experimental and Scaled Simulation Results for the Second Mode of the Smart Fin

\begin{tabular}{cccc}
\hline Actuator & Exp. Peak FRF $\left(\mathrm{mm} / \mathrm{s}^{2}\right)$ & FEM Peak FRF $\left(\mathrm{mm} / \mathrm{s}^{2}\right)$ & Difference $(\%)$ \\
\hline 1 & 8.35 & 9.79 & 17 \\
2 & 3.63 & 3.38 & -7 \\
3 & 9.96 & 10.46 & 5 \\
4 & 11.90 & 14.51 & 22 \\
5 & 1.61 & 1.25 & -23 \\
6 & 2.19 & 1.91 & -13 \\
7 & 1.92 & 2.18 & 14 \\
8 & 1.39 & 1.51 & 9 \\
9 & 2.77 & 2.31 & -16 \\
10 & 6.87 & 5.74 & -16 \\
11 & 7.58 & 6.10 & -20 \\
12 & 7.40 & 5.43 & -27 \\
Mean abs. diff. & - & - & 15.6 \\
Mean diff. & - & - & -4.6 \\
\hline
\end{tabular}

For example, according to the simulation, in the case of displacement control for the single actuator problem, the most effective actuator was at $\mathrm{x}=25 \mathrm{~mm}$ and $\mathrm{y}=20 \mathrm{~mm}$. This position is consistent with the first row of actuators, as determined by experiment. Specifically, it is equivalent to actuator 1 , which in the experimental test bed was determined to be the second most effective actuator. In the case of acceleration control for the single actuator problem, the most effective actuator according to simulation was at $\mathrm{x}=160 \mathrm{~mm}$ and $\mathrm{y}=125 \mathrm{~mm}$. This position, along the second node line and therefore representing a position of high control authority for the third mode, is equivalent to actuator number 10 in the oxperimental setup, which was determined to be the fifth most effective actuator. This discrepancy can be explained by the simulation condition that positions within $20 \mathrm{~mm}$ of the root were excluded in order to avoid interference with the fin root. This condition resulted in the first row (actuators 1-4 in experimental equivalent) not being considered as a possible optimal position for the single actuator. A removal of this condition from 
Table 5.6: Comparison of Experimental and Scaled Simulation Results for the Third Mode of the Smart Fin

\begin{tabular}{cccc}
\hline Actuator & Exp. Peak FRF $\left(\mathrm{mm} / \mathrm{s}^{2}\right)$ & FEM Peak FRF $\left(\mathrm{mm} / \mathrm{s}^{2}\right)$ & Difference (\%) \\
\hline 1 & 7.85 & 8.22 & 5 \\
2 & 5.16 & 6.06 & 17 \\
3 & 5.16 & 5.66 & 10 \\
4 & 4.29 & 4.98 & 16 \\
5 & 4.70 & 4.58 & -3 \\
6 & 3.91 & 4.44 & 14 \\
7 & 3.37 & 3.77 & 12 \\
8 & 3.36 & 3.50 & 4 \\
9 & 11.37 & 9.70 & -15 \\
10 & 6.82 & 6.73 & -1 \\
11 & 4.69 & 4.98 & 6 \\
12 & 4.64 & 4.31 & -7 \\
Mean abs. diff. & - & - & 9.1 \\
Mean diff. & - & - & 4.8 \\
\hline
\end{tabular}

the simulation shows an optimal placement within $20 \mathrm{~mm}$ of the root and consistent with experimental results. 
Table 5.7: Actuator Ranking from Most Effective to Least For Individual Modal Control

\begin{tabular}{|c|c|c|c|c|c|c|}
\hline Rank & $\begin{array}{c}\text { Mode } 1 \\
\text { Exp. }\end{array}$ & $\begin{array}{c}\text { Mode } 1 \\
\text { FEM }\end{array}$ & $\begin{array}{c}\text { Mode } 2 \\
\text { Exp. }\end{array}$ & $\begin{array}{c}\text { Mode } 2 \\
\text { FEM }\end{array}$ & $\begin{array}{c}\text { Mode } 3 \\
\text { Exp. }\end{array}$ & $\begin{array}{c}\text { Mode } 3 \\
\text { FEM }\end{array}$ \\
\hline $1 \mathrm{st}$ & 3 & 2 & 4 & 4 & 9 & 9 \\
\hline 2nd & 2 & 3 & 3 & 3 & 1 & 1 \\
\hline $3 \mathrm{rd}$ & 1 & 1 & 1 & 1 & 10 & 10 \\
\hline 4 th & 4 & 4 & 11 & 11 & 2 & 2 \\
\hline 5 th & 6 & 6 & 12 & 10 & 3 & 3 \\
\hline 6 th & 7 & 7 & 10 & 12 & 5 & 4 \\
\hline 7 th & 8 & 5 & 2 & 2 & 11 & 11 \\
\hline 8 th & 5 & 8 & 9 & 9 & 12 & 5 \\
\hline 9th & 10 & 10 & 6 & 7 & 4 & 6 \\
\hline 10th & 11 & 11 & 7 & 6 & 6 & 12 \\
\hline 11th & 12 & 12 & 5 & 8 & 7 & 7 \\
\hline 12 th & 9 & 9 & 8 & 5 & 8 & 8 \\
\hline
\end{tabular}

Table 5.8: Actuator Ranking from Most Effective to Least For Multi-Modal Control

\begin{tabular}{ccccc}
\hline Rank & $\begin{array}{c}\text { Acceleration } \\
\text { Exp. }\end{array}$ & $\begin{array}{c}\text { Acceleration } \\
\text { FEM }\end{array}$ & $\begin{array}{c}\text { Displacement } \\
\text { Exp. }\end{array}$ & $\begin{array}{c}\text { Displacement } \\
\text { FEM }\end{array}$ \\
\hline \hline 1st & 1 & 4 & 3 & 3 \\
2nd & 3 & 1 & 1 & 1 \\
3rd & 4 & 3 & 2 & 2 \\
4th & 2 & 2 & 4 & 4 \\
5th & 10 & 10 & 6 & 6 \\
6 th & 9 & 9 & 7 & 7 \\
7 th & 11 & 11 & 8 & 5 \\
8th & 12 & 12 & 5 & 8 \\
9 th & 6 & 6 & 10 & 10 \\
10 th & 7 & 7 & 11 & 11 \\
11th & 5 & 5 & 12 & 12 \\
12th & 8 & 8 & 9 & 9 \\
\hline
\end{tabular}


Table 5.9: Comparison of Actuator Ranking of Multiple Actuator Optimization with Experimental Results for Individual Modal Control using Equivalent Actuators

\begin{tabular}{|c|c|c|c|c|c|c|}
\hline & Mode 1 & Mode 1 & Mode 2 & Mode 2 & Mode 3 & Mode 3 \\
\hline Rank & Equiv. FEM & Exp. & Equiv. FEM & Exp. & Equiv. FEM & Exp. \\
\hline 1 st & 1 & 3 & 4 & 4 & 1 & 9 \\
\hline $2 \mathrm{nd}$ & 2 & 2 & 1 & 3 & 9 & 1 \\
\hline 3rd & 3 & 1 & 3 & 1 & 10 & 10 \\
\hline 4 th & 5 & 4 & 11 & 11 & 8 & 2 \\
\hline 5 th & 6 & 6 & 12 & 12 & 3 & 3 \\
\hline
\end{tabular}

Table 5.10: Comparison of Actuator Ranking of Multiple Actuator Optimization with Experimental Results for Multi-Modal Control using Equivalent Actuators

\begin{tabular}{ccccc}
\hline Rank & $\begin{array}{c}\text { Acceleration } \\
\text { Equiv. FEM }\end{array}$ & $\begin{array}{c}\text { Acceleration } \\
\text { Exp. }\end{array}$ & $\begin{array}{c}\text { Displacement } \\
\text { Equiv. FEM }\end{array}$ & $\begin{array}{c}\text { Displacement } \\
\text { Exp. }\end{array}$ \\
\hline \hline 1st & 1 & 1 & 1 & 3 \\
2nd & 3 & 3 & 3 & 1 \\
3rd & 4 & 4 & 2 & 2 \\
4th & 9 & 2 & 4 & 4 \\
5th & 10 & 10 & 5 & 6 \\
\hline
\end{tabular}




\section{Chapter 6}

\section{Conclusions and Recommendations}

\subsection{Conclusions}

The combination of using a finite element model to obtain FRFs for the first three modes of the fin and a genetic algorithm solver provided an efficient means of optimizing the configuration of actuators on the fin. The position of a single actuator or sensor pair can be optimized by the method described in Chapter 3. Alternatively, the configuration of multiple actuators can be simultaneously optimized using the method described in Chapter 4.

As consistent with the bending node lines shown in Figure 2.1, high bending actuation authority was achieved for the first mode by placement of actuators near the first node line at the root and for the third mode near both the first and the second bending node lines. Similarly, high torsional actuation authority for the second mode was achieved by placement of actuators near the leading and trailing edges as far as possible from the central node line running from root to tip of the fin. The optimized configurations for individual modal control placed the actuators to satisfy these conditions. However, with multi-modal acceleration and displacement control, the optimized configurations were trade-offs between the actuator positions used for individual modal control. The first mode dominated in displacement, and the optimal actuator configurations were similar to the configuration for control of the first mode. Conversely, the third mode dominated in acceleration, and the optimal actuator configurations were similar to the configuration for control of the third 
mode. However, in both acceleration and displacement, the non-dominant modes were still significant in influencing the optimum configurations.

The simulation results were verified using a comparison to results obtained from the experimental model of the flexible fin. The optimized configurations obtained through simulation and experiment were quite consistent, and the FRF peak magnitudes also matched well once the second and third modes were scaled using suitable modal scaling factors.

A number of new ideas were developed in this work:

- The method of using finite element FRFs to derive the fitness function for subsequent optimization using a genetic algorithm is a novel approach whose principal benefit lies in the decoupling of the finite element method and genetic algorithm solver. This allows for the solution of problems with practically any degree of complexity without necessitating the derivation of a closed-form analytical solution.

- Using a genetic algorithm for three-dimensional polynomial surface fitting is a novel approach that takes advantage of a genetic algorithm's ability to process large amounts of trial solutions to converge over a number of generations to an optimal solution. Although it would have been possible to apply a closed form regression, the implementation would have been prohibitively cumbersome, particularly with a ninth order three-dimensional polynomial surface. Sub-dividing the region into sub-areas for a better surface optimization, although reminiscent of the two-dimensional method of splines, was an approach developed specifically for this thesis.

- Many of the features of the surface fitting genetic algorithm were novel. The introduction of a range parameter which simultaneously provided bounds for synthesis of an initial population pool and limits for mutation was particularly effective. The mutation operator was specifically designed for polynomial surface fitting. As such, it was able to maintain the fine balance of providing sufficiently large mutations to introduce significant change at most generations without reducing the fitness to the point that the mutation would automatically be rejected at every generation. The reproduction and immigration operators were also designed specifically for the surface 
fitting algorithm. The reproduction operator was more complex than in most genetic algorithms. The use of an immigration operator to increase the convergence rate is rare in genetic algorithms, but in this case it proved effective.

- The multi-modal fitness functions developed in this thesis are effective for obtaining a degree of flexibility in the optimization for control of vibration under various operating conditions. Their simplicity is an asset, and customization can be achieved through the use of the modal weighting factors.

- The ability to optimize the configuration of any number of actuators on the smart fin using a genetic algorithm is probably the most practical result of this thesis. The results can also be used in a trade-off study to determine the optimum configuration of actuators consistent with a particular set of costs and benefits.

- The parameters Minimum Effectiveness (ME) and Relative Mean Effectiveness (RME) were developed in this thesis to provide a measure of relative actuator contribution and configuration efficiency. These parameters can be applied to any geometry or configuration, and could be used with any multiple actuator or sensor optimization.

\subsection{Recommendations}

This section outlines some recommendations for future work:

- A graphical user interface could be developed for the polynomial surface fitting genetic algorithm code in order to make it more user friendly. The code could also be modified to increase its effectiveness for general polynomial surface fitting. The code is currently theoretically capable of fitting any set of data in space to a polynomial surface, but in practice this can be prohibitively difficult if the data is extremely erratic.

- An approach to quantify damping that is more consistent with the higher vibration modes would be useful. In this thesis, an overall structural damping ratio of $3.4 \%$ was adopted in order to ensure consistency between experimental and simulation results 
for the first mode. This led to scaling inconsistencies with the second and third mode FRF magnitudes. Although this had only a minor effect on the actual optimization, in most cases it will be difficult to determine structural damping through simulation alone. This makes modal scaling nearly impossible to implement, and in effect, assigns poorly understood weighting factors to the fitness function.

- The method for optimizing the configuration of multiple actuators on the flexible fin used many passive actuators. This should not have affected the optimization results significantly because the general relationship between FRFs would have been relatively consistent. However, the absolute FRF magnitudes might have been significantly altered by this, and this could have contributed to additional modal scaling discrepancies. Ideally, a method which does not rely on passive actuators would be better able to account for this.

- The development of more comprehensive benefits and costs would be necessary to implement a full scale trade-off study in order to optimize the configuration of a variable number of actuators on the smart fin. This would have to be consistent with minimal control authority requirements and expected operating conditions in order to be applicable to configuration optimization.

- The definition of actuation authority could be expanded to incorporate additional accelerometers, taking into account the vibration of multiple points on the fin simultaneously. This would better reflect fin excitation, although the resulting fitness function would need to be consistent with modal emphasis and the fact that different points on the fin are more susceptible to excitation by particular modes. Thus, a multi-accelerometer fitness function would exacerbate the challenge of modal scaling.

- A study of expected operating conditions for the fin and resulting actuation authority requirements for all excited modes would be necessary to achieve an optimal configuration that is consistent with actual anticipated loading conditions. This study should be used to develop appropriate modal weighting factors for the fitness function. An 
optimization would need to be applied to a full scale aircraft fin (experimental and/or simulation) before the configuration could be implemented on an actual aircraft. 


\section{List of References}

[1] T. Ryall, R. Moses, M. Hopkins, D. Henderson, D. Zimcik, and F. Nitzsche, "Buffet load alleviation," in Second Australian Congress on Applied Mechanics, Canberra, Australia, pp. 126-131, 1999.

[2] F. Nitzsche, D. Zimcik, and K. Langille, "Active control of vertical fin buffeting with aerodynamic control surface and strain actuation," in 38th Structures, Structural Dynamics, and Materials Conference, Adaptive Structures Forum, AIAA-971386, Kissimmee, FL, pp. 1467-1477, 1997.

[3] A. Yousefi-Koma, A. Chen, and D. Zimcik, "Development of an active control system for a smart fin," in 7th CANSMART International Workshop on Smart Materials and Structures, Montreal, Canada, 2004.

[4] A. Yousefi-Koma, D. Zimcik, and A. Mander, "Experimental and theoretical system identification of flexible structures with piezoelectric actuators," in 24th International Congress of the Aeronautical Sciences (ICAS), Yokohama, Japan, 2004.

[5] L. Meyn and K. James, "Full-scale wind-tunnel studies of f/a-18 tail buffet," Journal of Aircraft, vol. 33 (3), pp. 589-595, 1996.

[6] R. Moses and E. Pendleton, "A comparison of pressure measurements between a fullscale and 1/6-scale twin tail during buffet," in 83rd Structures and Materials Panel (SMP) Meeting, Loads and Requirements for Military Aircraft: AGARD-R-815, NATO Advisory Group for Aerospace Research and Development, 1999.

[7] K. Lazarus, E. Saarmaa, and G. Agnes, "An active smart material system for buffet load alleviation," in Second SPIE Conference on Smart Structures and Material Systems: Industrial and Commercial Applications of Smart Structures Technologies, San Diego, CA, pp. 179-192, 1995.

[8] H. Ashley, S. Rock, S. Digurmarthi, R. Chaney, and A. Eggers, "Active control for fin buffet alleviation," in Wl-TR-93-3099, Air Force Wright Laboratory, 1994.

[9] R. Hauch, J. Jacobs, C. Dima, and K. Ravindra, "Reduction of vertical tail buffet response using active control," Journal of Aircraft, vol. 33 (3), pp. 617-622, 1996. 
[10] J. Heeg, "Analytical and experimental investigation of flutter suppression by piezoelectric actuation," NASA Technical paper 3241, 1993.

[11] F. Nitzsche, D. Zimcik, and D. Liberatore, "Finite element approach for the design of control algorithms for vertical fin buffeting using strain actuation," in RTO AVT Symposium on Active Control Technology for Enhanced Performance Operational Capabilities of Military Aircraft, RTO MP-051, Kingston, Canada, pp. 8.1-8.10, 2000.

[12] B. Bailey, Investigation of a Composite Hingeless Helicopter Rotor Blade with Integral Actuators (Masters Thesis). Ottawa, ON, Canada: Carleton University, 2000.

[13] A. Bent, Active Fibre Composites for Structural Actuation (PhD Thesis). Cambridge, MA, USA: Massachusetts Institute of Technology, 2000.

[14] R. Clark, W. Saunders, and G. Gibbs, Adaptive Structures: Dynamics and Control. New York, NY, USA: John Wiley and Sons, 1998.

[15] D. Goldberg, Genetic Algorithms in Search, Optimization, and Machine Learning. Reading, MA, USA: Addison Wesley, 1989.

[16] C. Karr, B.Weck, D. Massart, and P. Vankeerberghen, "Least median squares curve fitting using a genetic algorithm," Engineering Applications of Artificial Intelligence, vol. 8 (2), pp. 177-189, 1995.

[17] M. Jamshidi, L. Coelho, R. Krohling, and P. Fleming, Robust Control Systems with Genetic Algorithms. New York, NY, USA: CRC Press, 2003.

[18] S. Wang, S. Quek, and K. Ang, "Vibration control of smart piezoelectric composite plates," Smart Materials and Structures, vol. 10, pp. 637-644, 2001.

[19] M. Kwak, S. Heo, and S. Han, "Optimal configuration of piezoceramic sensors and actuators for the vibration control of rectangular plate," in 14th International Conference on Adaptive Structures and Technologies (ICAST), Seoul, Korea, 2003.

[20] L. Sheng and K. Kapania, "Genetic algorithms for optimization of piezoelectric actuator locations," AIAA Journal, vol. 39 (9), pp. 1818-1822, 2002.

[21] V. Lopes, V. Steffen, and D. Inman, "Active vibration control using piezoelectric sensor/actuator patches for vibration control," Intelligent Material Systems and Structures, 2005.

[22] C. Cook and W. Crossley, "Investigation of genetic algorithm approaches for smart actuator placement for aircraft maneuvering," in 39th AIAA Aerospace Sciences Meeting and Exhibit, Reno, NV, 2001. 
[23] M. Rahmoune, M. Benjeddou, A. Ohayon, and D. Osmont, "Finite element modeling of a smart structure plate system," in 7th International Conference on Adaptive Structures, Rome, Italy, 1996.

[24] S. Rao, Mechanical Vibrations (4th Ed.). Upper Saddle River, NJ, USA: Prentice Hall, 2004.

[25] I. Gonos, N. Mastorakis, and M. Swamy, "Genetic algorithm approach to the problem of factorization of general multidimensional polynomials," IEEE Transactions on Circuits and Systems I: Fundamental Theory and Aplication, vol. 50 (1), pp. 16-22, 2003.

[26] C. Karr, D. Stanley, and B. Scheiner, "A genetic algorithm aplied to least squares curve fitting," Report of Investigations No. 9339 U.S. Department of the Interior, Bureau of Mines, Washington D.C., 1991.

[27] Mathworks, Evaluating the Goodness of Fit. Matlab Mathworks Website: Curve Fitting Toolbox, 2004.

[28] M. Moed, "Reducing the search time of a steady state genetic algorithm using the immigration operator," in Third International Conference on Tools for Artificial Intelligence, pp. 500-501, 1992. 


\section{Appendix A}

\section{Surface Fitting Matlab Code}

$\%$ This algorithm fits a 2-variable n-order polynomial $(z=f(x, y))$ to

m data points by a genetic algorithm method

$\%$ get data points

xvalues $=[$ (user defined $)] ;$ yvalues $=[$ (user defined $)]$;

functionvalues $=[$ (user defined $)]$;

reenter $=1$;

while reenter $==1$

$\%$ Select order of polynomial

$\mathrm{n}$ = input('What order of polynomial do you wish to use? (min 1, $\max 9$, default 9): ');

if isempty(n)

$\mathrm{n}=9$;

end 


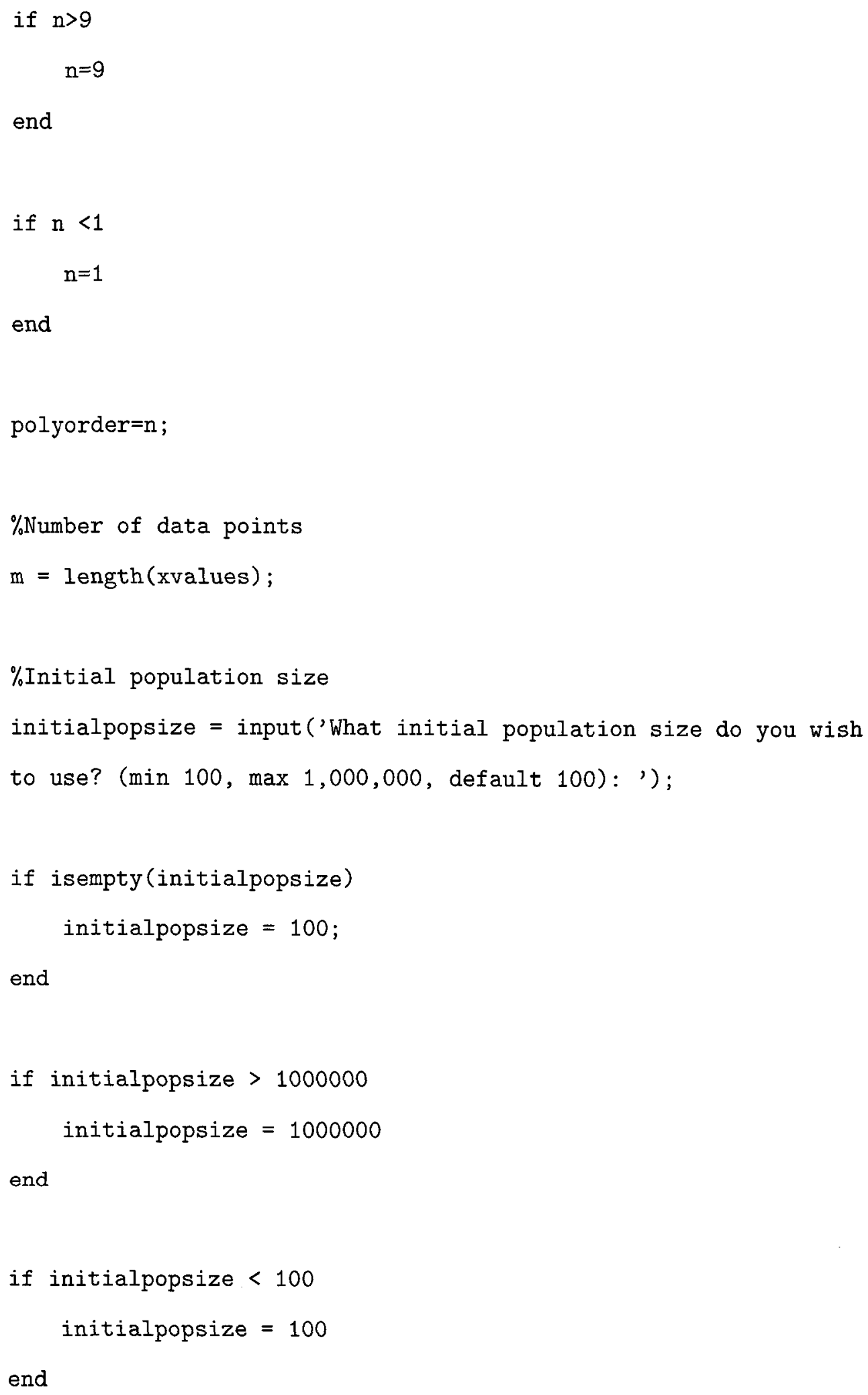




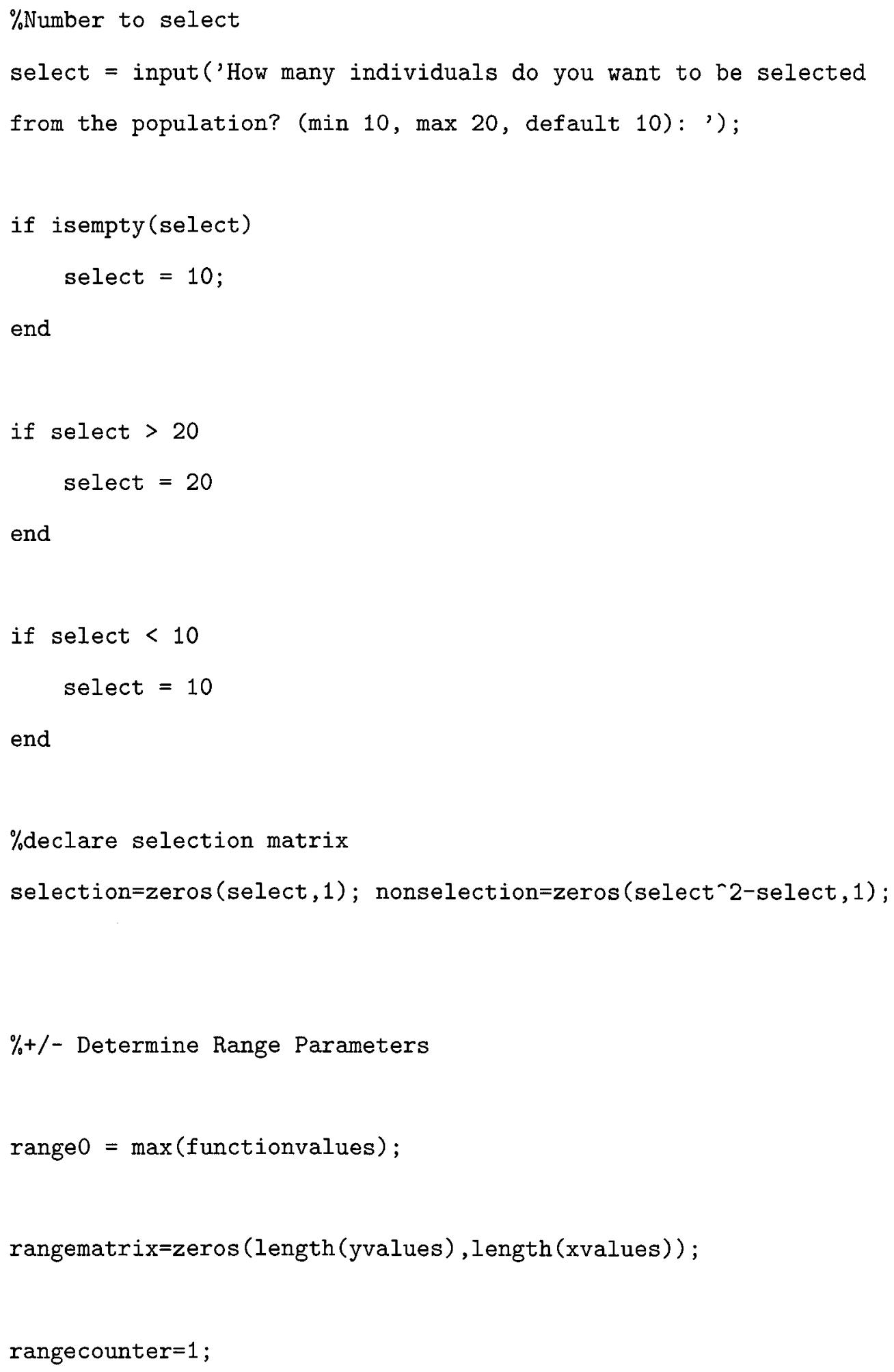




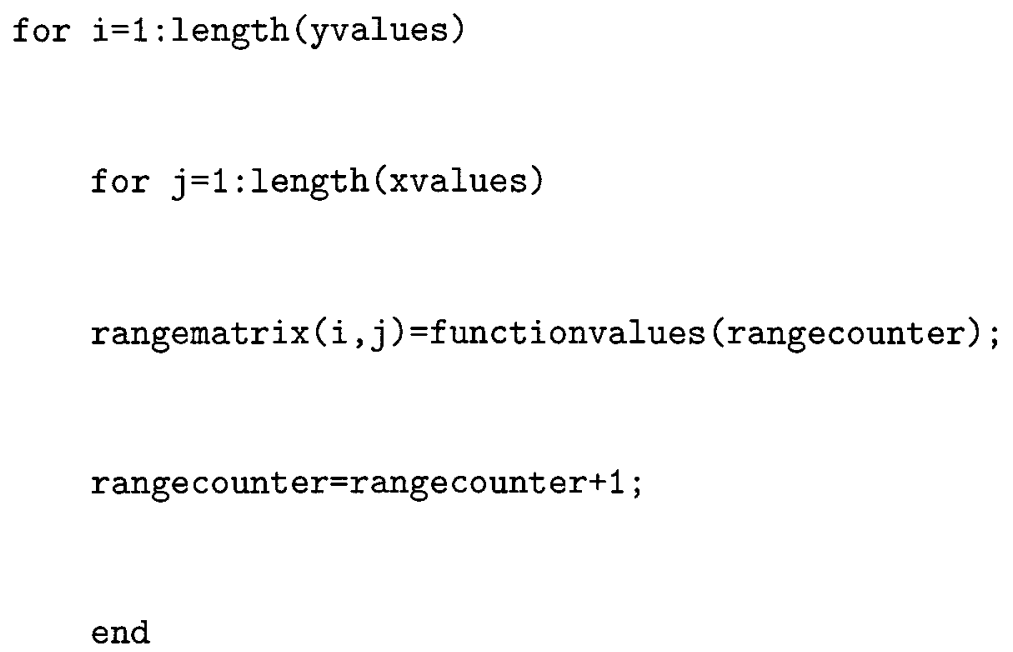


rangematrix $1 y(i, j)=$ rangematrix $(i, j)-\operatorname{rangematr} i x(i+1, j)$;

end

end

range $1=\operatorname{abs}(\max (\operatorname{rangematrix} 1 x))$;

if $\operatorname{abs}(\max (\operatorname{rangematrix} 1 \mathrm{y}))>\operatorname{abs}(\max (\operatorname{rangematrix} 1 \mathrm{x}))$

range $1=\operatorname{abs}(\max ($ rangematrix $1 \mathrm{y})$

end

rangematrix $2 x=$ zeros (length (yvalues), length (xvalues)-2);

rangematrix $2 y=z e r o s$ (length (yvalues) -2 , length (xvalues));

for $i=1:$ length (yvalues)

for $j=1:$ length (xvalues) -2

rangematrix $2 x(i, j)=\operatorname{rangematrix} 1 x(i, j)-\operatorname{rangematrix} 1 x(i, j+1) ;$

end

end 


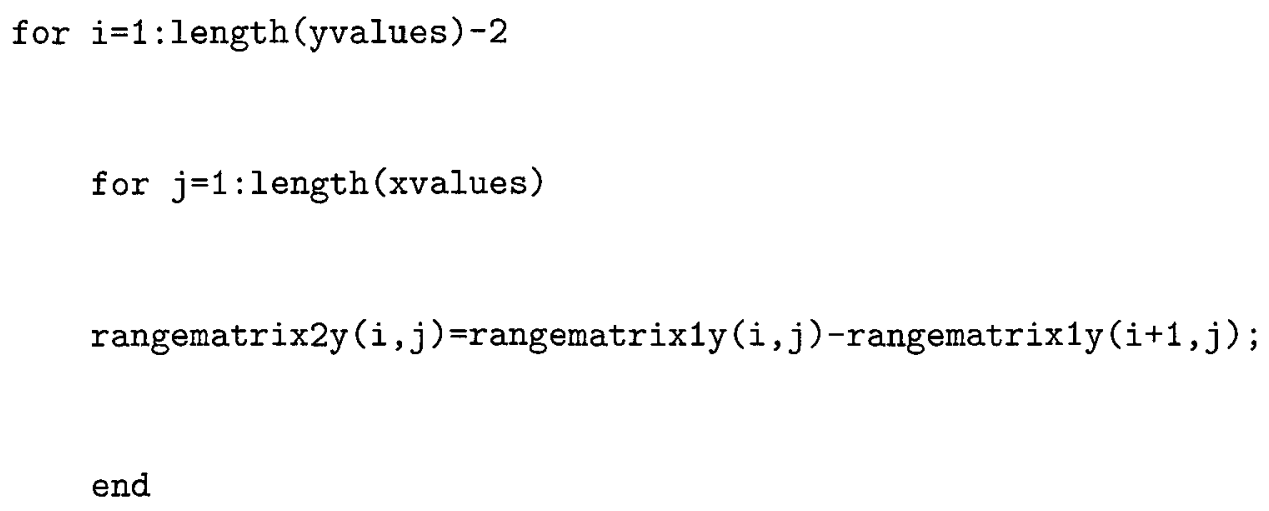


end

if mutatechance > 50

mutatechance $=50$

end

if mutatechance $<1$

mutatechance $=1$

end

$\%$ Mutation severity

mutateseverity $=$ input ('What is severity of mutation? (min 0.01, $\max 1$, default 0.1 ): ');

if isempty(mutateseverity)

mutateseverity $=0.1$

end

if mutateseverity $>1$

mutateseverity $=1$

end

if mutateseverity $<0.01$

mutateseverity $=0.01$

end

$\%$ Max error

checkerror $=$ input ('What is the maximum allowable error? (use a higher value for higher order polynomials; min 0.1, $\max 10,000$, 


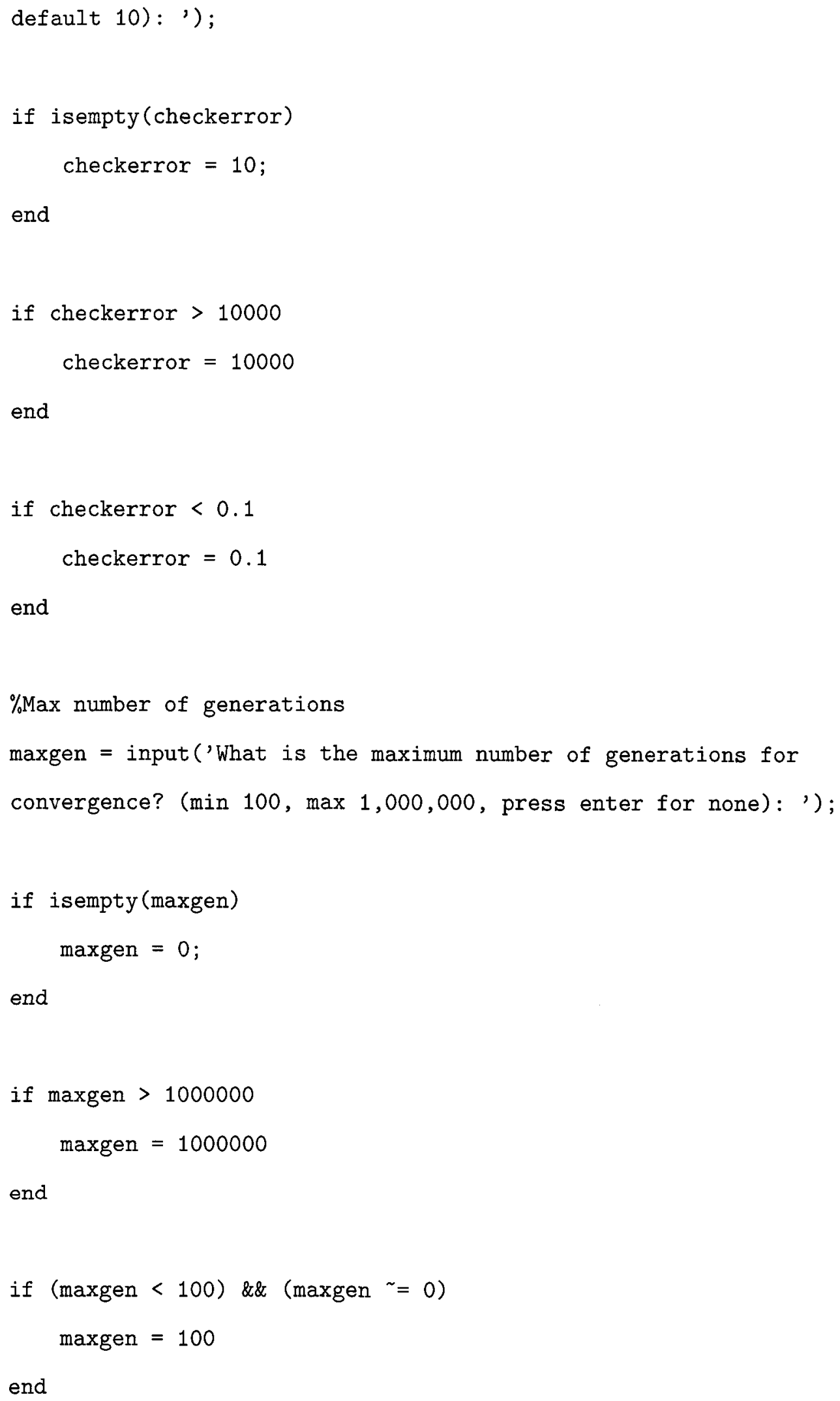




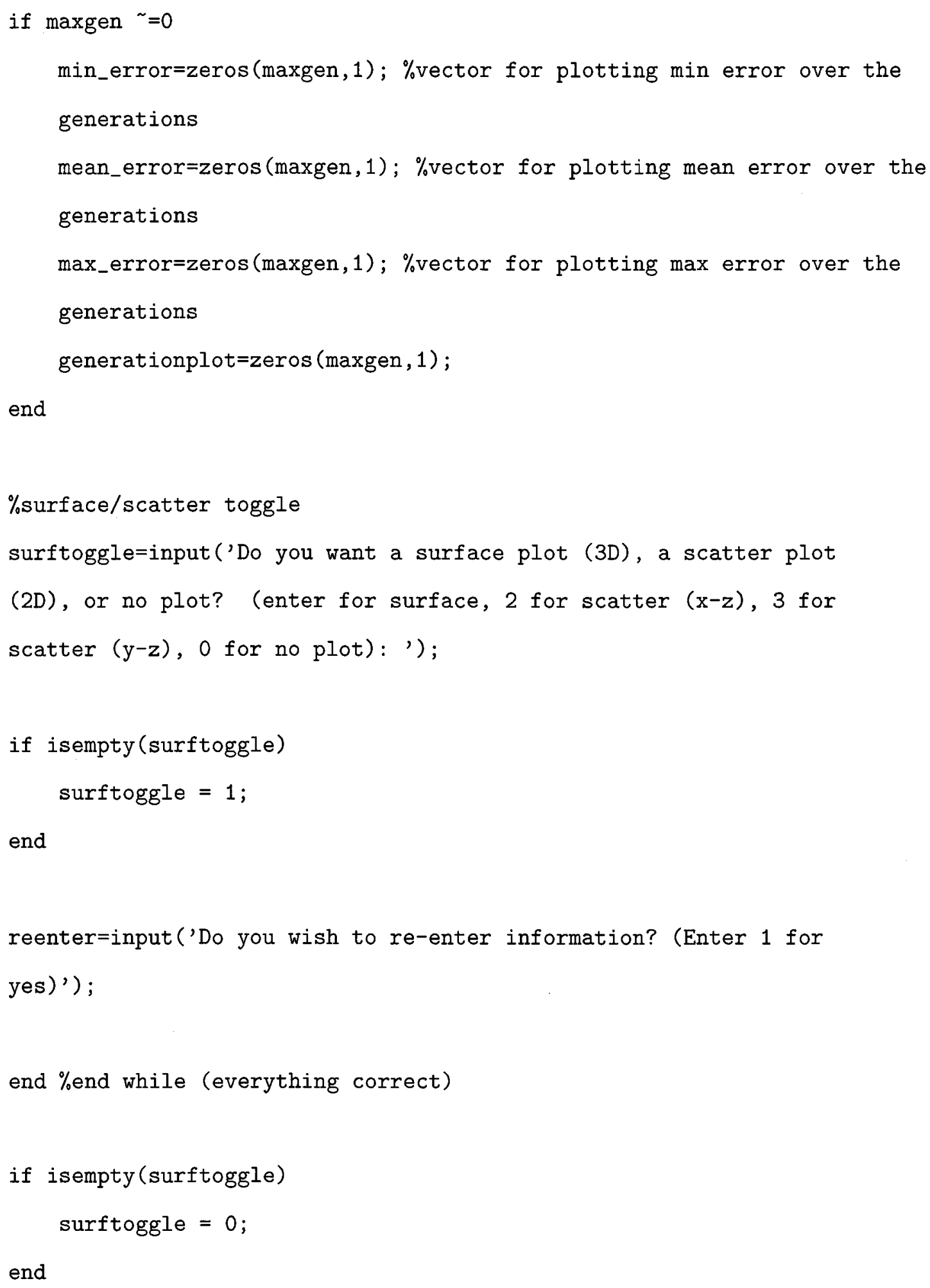




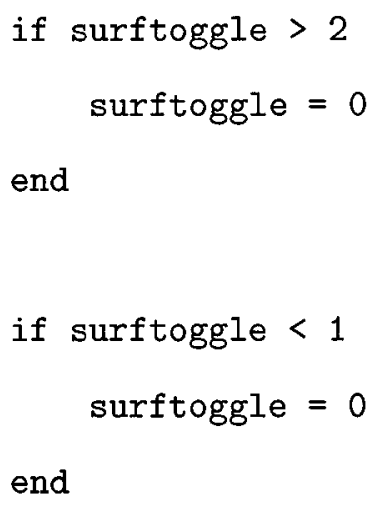

end

if surftoggle $<1$

surftoggle $=0$

end

\%DEFINE MATRICES

trialvalues=zeros (initialpopsize,m); \%the trial function values at each point

$\%$ define coefficient matrix coeffs=zeros(initialpopsize,55); \%55 is number of coefficients for 9th order polynomial

\%define error matrix error=zeros (initialpopsize,1); \%square root of sum of squares errorsum $=0 ; \%$ square root of sum of squares

\%assign initial random values to coefficient matrix:

for $j=1$ :initialpopsize

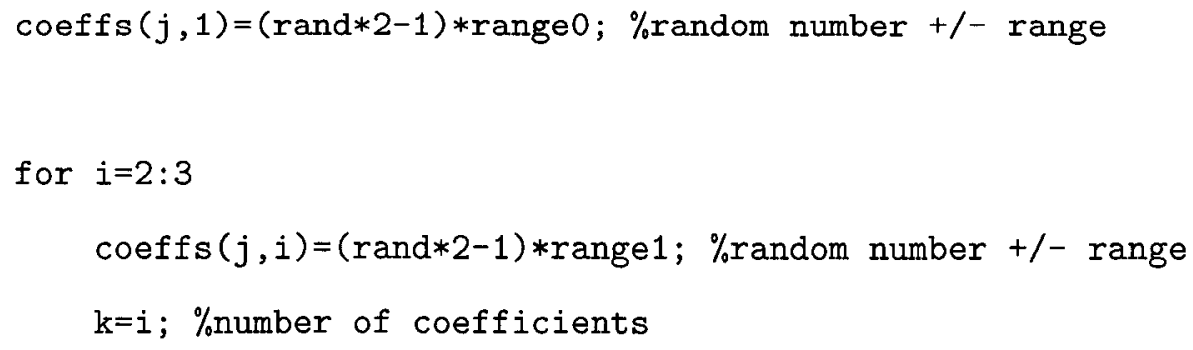


end

if $n>1$

for $i=4: 6$

coeffs $(j, i)=($ rand $* 2-1) *$ range $2 ; \%$ random number $+/-$ range

$\mathrm{k}=\mathrm{i}$; $\%$ number of coefficients

end

end

if $n>2$

for $i=7: 10$

coeffs $(j, i)=($ rand $* 2-1) * r$ ange $3 ; \%$ random number $+/-$ range

$k=i ; \%$ number of coefficients

end

end

if $n>3$

for $i=11: 15$

coeffs $(j, i)=($ rand $* 2-1) *$ range $3 ; \%$ random number $+/-$ range

$k=i ; \%$ number of coefficients

end

end

if $\mathrm{n}>4$

for $i=16: 21$

coeffs $(j, i)=($ rand $* 2-1) *$ range $3 ; \%$ random number $+/-$ range

$\mathrm{k}=\mathrm{i}$; $\%$ number of coefficients

end

end 


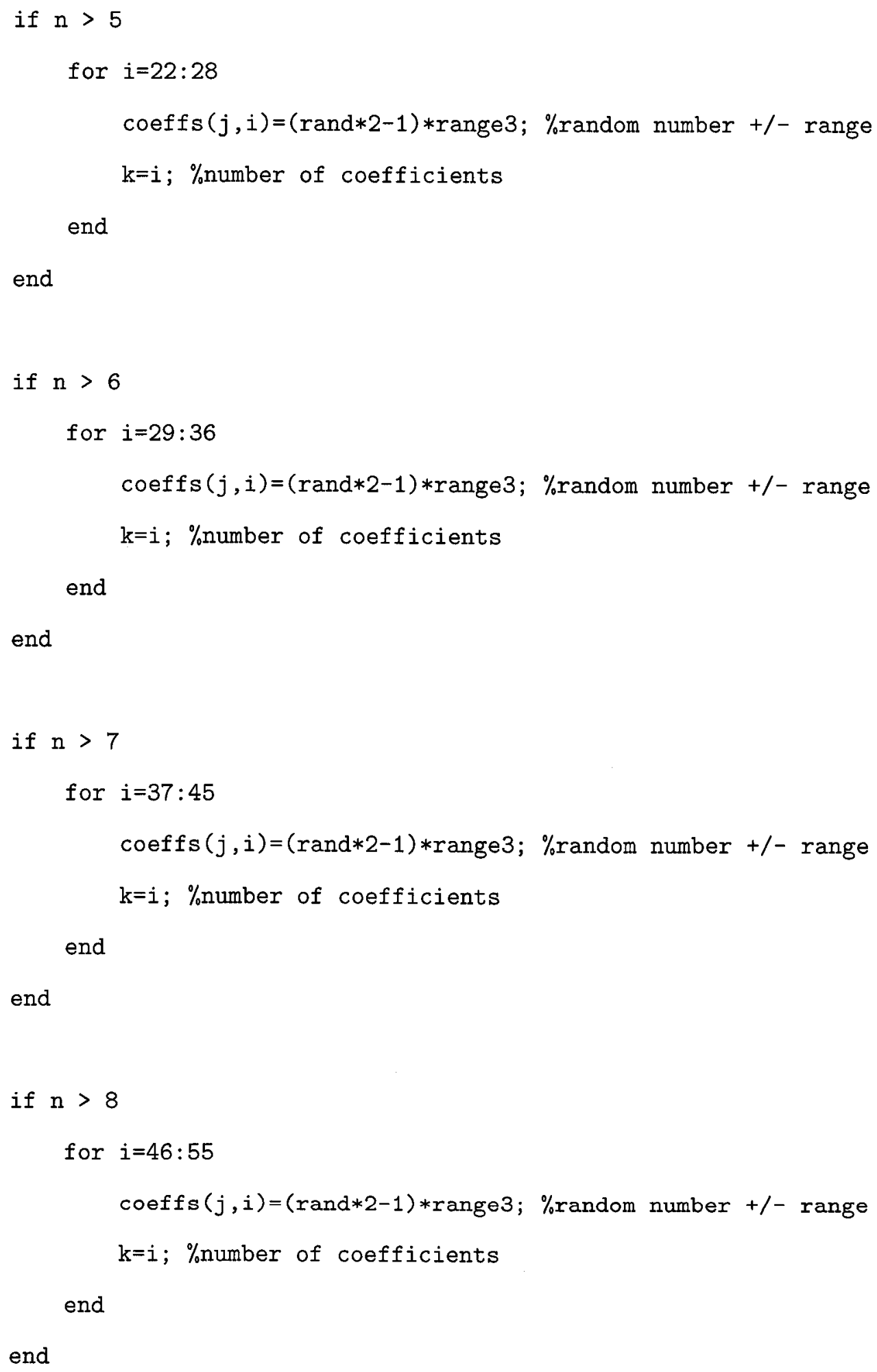


end

mutatedcoeffs=zeros $(1,55) ; \%$ trial coeff set

\%determine fitness: square root of sum of squared errors

$\%$ first we need to evaluate the function value

for $j=1$ :initialpopsize

for $i=1: m$

$\operatorname{trialvalues}(j, i)=\operatorname{coeffs}(j, 1)+(\operatorname{coeffs}(j, 2) * \operatorname{xvalues}(i))+$

$(\operatorname{coeffs}(j, 3) * \operatorname{yvalues}(i))+(\operatorname{coeffs}(j, 4) * \operatorname{xvalues}(i)-2)+$

$(\operatorname{coeffs}(j, 5) * \operatorname{xvalues}(i) * \operatorname{yvalues}(i))+\left(\operatorname{coeffs}(j, 6) * \operatorname{yvalues}(i)^{\wedge} 2\right)+$

$\left(\operatorname{coeffs}(j, 7) * \operatorname{xvalues}(i)^{-} 3\right)+\left(\operatorname{coeffs}(j, 8) * \operatorname{xvalues}(i)^{\wedge} 2 * \operatorname{yvalues}(i)\right)+$

$(\operatorname{coeffs}(j, 9) * \operatorname{xvalues}(i) * \operatorname{yvalues}(i) \sim 2)+(\operatorname{coeffs}(j, 10) * \operatorname{yvalues}(i) \sim 3)+$

$\left(\operatorname{coeffs}(j, 11) * x v a l u e s(i)^{\wedge} 4\right)+\left(\operatorname{coeffs}(j, 12) * \operatorname{xvalues}(i)^{\wedge} 3 * \operatorname{yvalues}(i)\right)+$

$\left(\operatorname{coeffs}(j, 13) * \operatorname{xvalues}(i)^{\wedge} 2 * \operatorname{yvalues}(i)^{\sim} 2\right)+(\operatorname{coeffs}(j, 14) * \operatorname{xvalues}(i) *$

yvalues $(i)-3)+(\operatorname{coeffs}(j, 15) * \operatorname{yvalues}(i) \sim 4)+(\operatorname{coeffs}(j, 16) *$

xvalues (i $\left.)^{\wedge} 5\right)+\left(\operatorname{coeffs}(j, 17) * \operatorname{xvalues}(i)^{\wedge} 4 *\right.$ yvalues $\left.(i)\right)+$

$\left(\operatorname{coeffs}(j, 18) * x \operatorname{xalues}(i)^{\wedge} 3 * \operatorname{yvalues}(i)^{\wedge} 2\right)+(\operatorname{coeffs}(j, 19)$

*xvalues (i)^ $2 * \operatorname{yvalues}(i) \wedge 3)+(\operatorname{coeffs}(j, 20) * \operatorname{xvalues}(i) * \operatorname{yvalues}(i) \sim 4)+$

$\left(\operatorname{coeffs}(j, 21) * \operatorname{yvalues}(i)^{\wedge} 5\right)+\left(\operatorname{coeffs}(j, 22) * \operatorname{xvalues}(i)^{\wedge} 6\right)+$

$\left(\operatorname{coeffs}(j, 23) * \operatorname{xvalues}(i)^{\wedge} 5 * \operatorname{yvalues}(i)\right)+(\operatorname{coeffs}(j, 24) * \operatorname{xvalues}(i) \wedge 4 *$

yvalues $\left.\left.(i)^{\wedge} 2\right)+(\operatorname{coeffs}(j, 25) * \operatorname{xvalues}(i))^{-3 * y v a l u e s}(i) \wedge-3\right)+$

$(\operatorname{coeffs}(j, 26) * \operatorname{xvalues}(i) \sim 2 * \operatorname{yvalues}(i) \wedge 4)+(\operatorname{coeffs}(j, 27) * \operatorname{xvalues}(i) *$

yvalues $\left.(i)^{\wedge} 5\right)+\left(\operatorname{coeffs}(j, 28) * \operatorname{yvalues}(i)^{\wedge} 6\right)+\left(\operatorname{coeffs}(j, 29) * \operatorname{xvalues}(i)^{\wedge} 7\right)+$

$\left(\operatorname{coeffs}(j, 30) * \operatorname{xvalues}(i)^{\wedge} 6 * \operatorname{yvalues}(i)\right)+\left(\operatorname{coeffs}(j, 31) * \operatorname{xvalues}(i)^{\wedge} 5 *\right.$

yvalues $\left.(i)^{\wedge} 2\right)+\left(\operatorname{coeffs}(j, 32) * \operatorname{xvalues}(i)^{\wedge} 4 * \operatorname{yvalues}(i)^{\wedge} 3\right)+(\operatorname{coeffs}(j, 33) *$

xvalues (i) ^ $3 *$ yvalues (i ) 4$)+\left(\operatorname{coeffs}(j, 34) * \operatorname{xvalues}(i)^{\wedge} 2 * \operatorname{yvalues}(i)^{\wedge} 5\right)+$

$(\operatorname{coeffs}(j, 35) * \operatorname{xvalues}(i) * \operatorname{yvalues}(i) \sim 6)+\left(\operatorname{coeffs}(j, 36) * \operatorname{yvalues}(i)^{\sim} 7\right)+$

$\left(\operatorname{coeffs}(j, 37) * \operatorname{xvalues}(i)^{\wedge} 8\right)+\left(\operatorname{coeffs}(j, 38) * \operatorname{xvalues}(i)^{\wedge} 7 * \operatorname{yvalues}(i)\right)+$ 


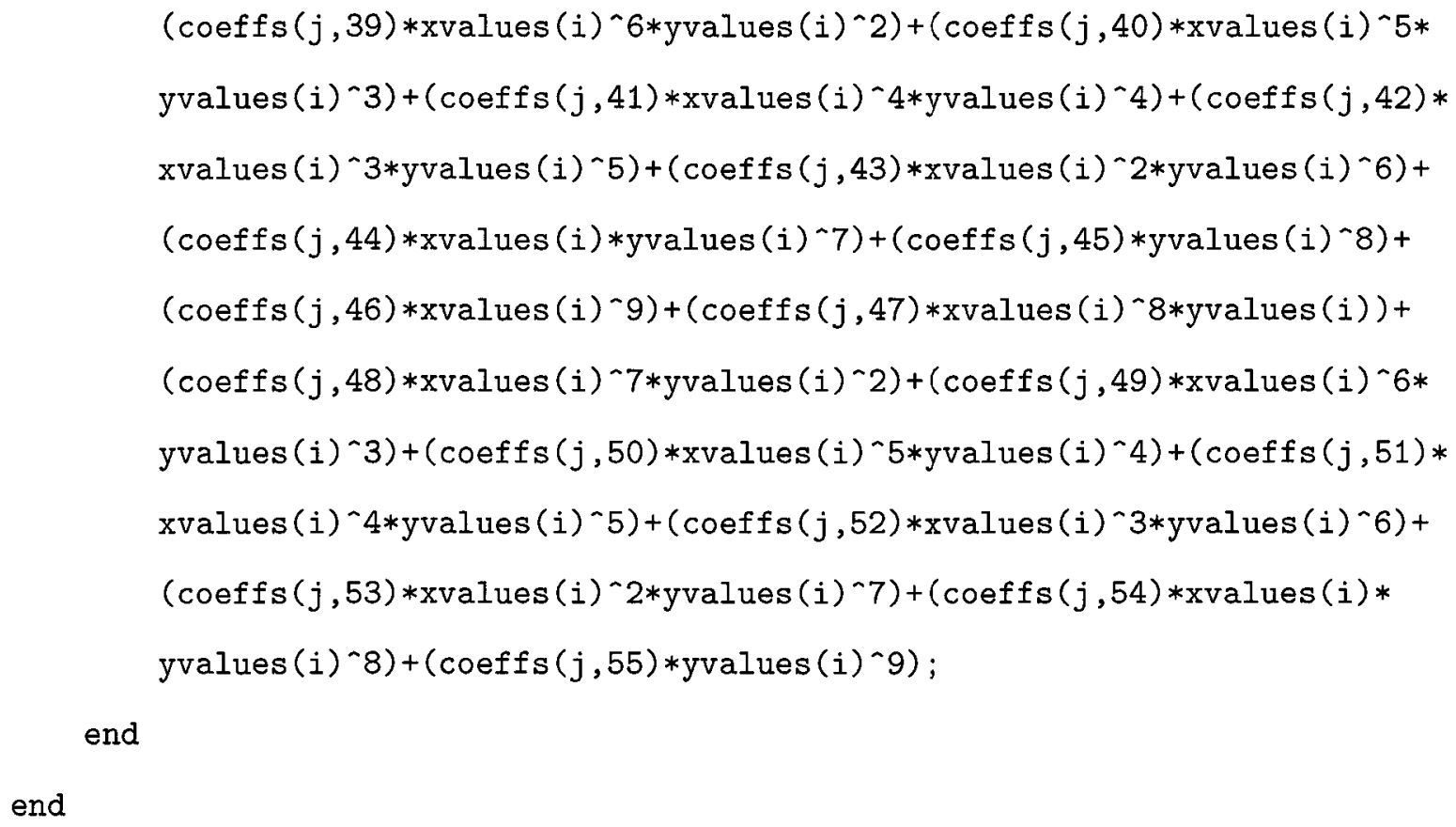


$\operatorname{error}(I)=$ inf; $\quad \%$ set the error of that individual to infinity so the loop will select the next lowest error, etc.

end $\%$ end for $i$

\%ake new population by breeding all posible combinations. Note that the \%parents are part of the population (self-cross), although they are $\%$ subject to mutation (if favourable)

newcoeffs=zeros (select`2,55); \%declare newpopulation matrix. Note for breeding all selected values, the number of possible $\%$ combinations is select 2 .

generation=1; \%initialize population counter generationplot (1)=1;

$\%-$ START OF LOOP

while (minimum_error $>$ checkerror \&\& (generation $<$ maxgen || maxgen $==0)$ )); \% Minimum absolute fitness for ending algorithm. Ideally should be zero.

generation=generation 1 ; \% Increase number of generations by 1 .

for $r=1:$ select $\%$ for each of the selected individuals primarycross=selection( $n$ ); \%set each one in turn as the primary cross individual

for $i=1:$ select $\%$ for each other selected individual (including itself for self cross). This is the secondary cross. level $=(r-1) *$ select $+i ; \%$ ind position in newpopulation matrix 
based on the primary and secondary cross.

alpha=rand $(55,1)$; \%result of cross for each allele, 0 to 1 for alpha, weighting on primary vs. secondary cross. for $j=1: 55 \%$ for each allele newcoeffs $($ level,$j)=\operatorname{alpha}(j) * \operatorname{coeffs}($ primarycross,$j)+$ (1-alpha $(j)) *$ coeffs (selection $(i), j) ; \%$ set allele to weighted value of primary cross and secondary cross end \%end for $j$

end \%end for $i$

end \%end for $r$

coeffs=zeros $\left(\right.$ select $\left.^{`} 2,55\right) ; \quad \%$ re-declare population matrix

(need to resize from popsize to select`2 if first run)

error=zeros (select`2,1); $\quad \%$ re-declare error matrix (need to re-size)

meanerror=zeros $($ select^2,1); $\quad \%$ declare mean error matrix

maxerror=zeros (select`2,1); \%declare max error matrix

trialvalues=zeros $\left(\right.$ select $\left.{ }^{\sim} 2, \mathrm{~m}\right) ; \quad \%$ re-size the trial function values matrix

mutatedvalues=zeros $(1, \mathrm{~m})$; $\quad \%$ for comparison with mutated values

coeffs=newcoeffs; $\quad \%$ set population to bred population

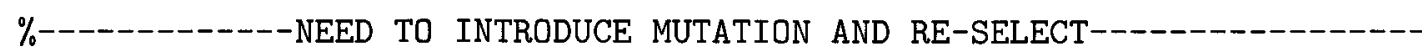

\%Introduce a chance of mutation in the population before selection

$\%$ (since most mutations will be detrimental and therefore selected out)

for $j=1$ : select $2 \%$ for whole pop 


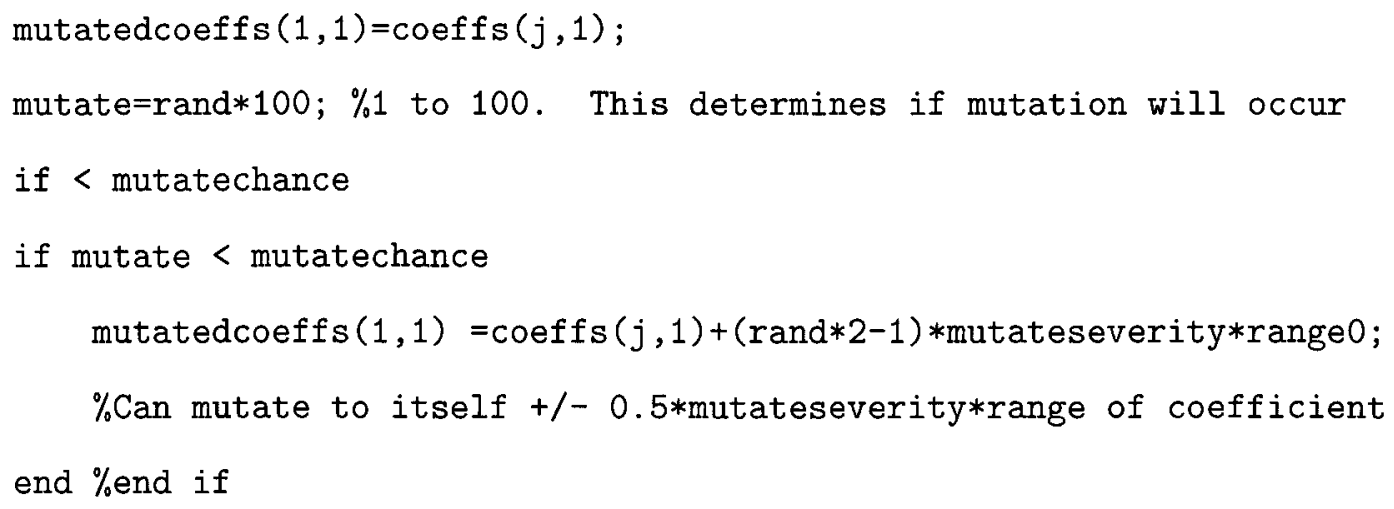


end \%end for $i$

end

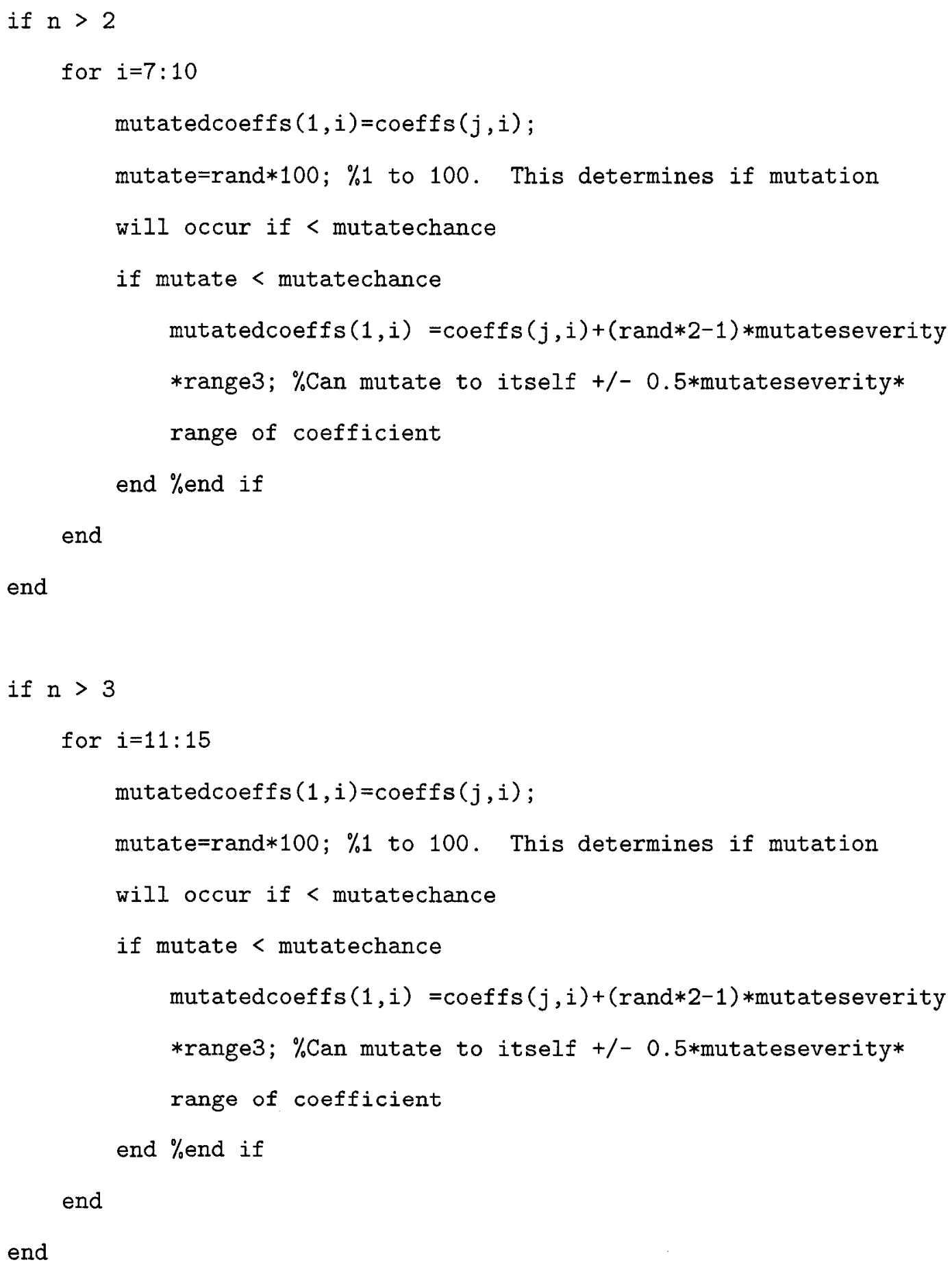




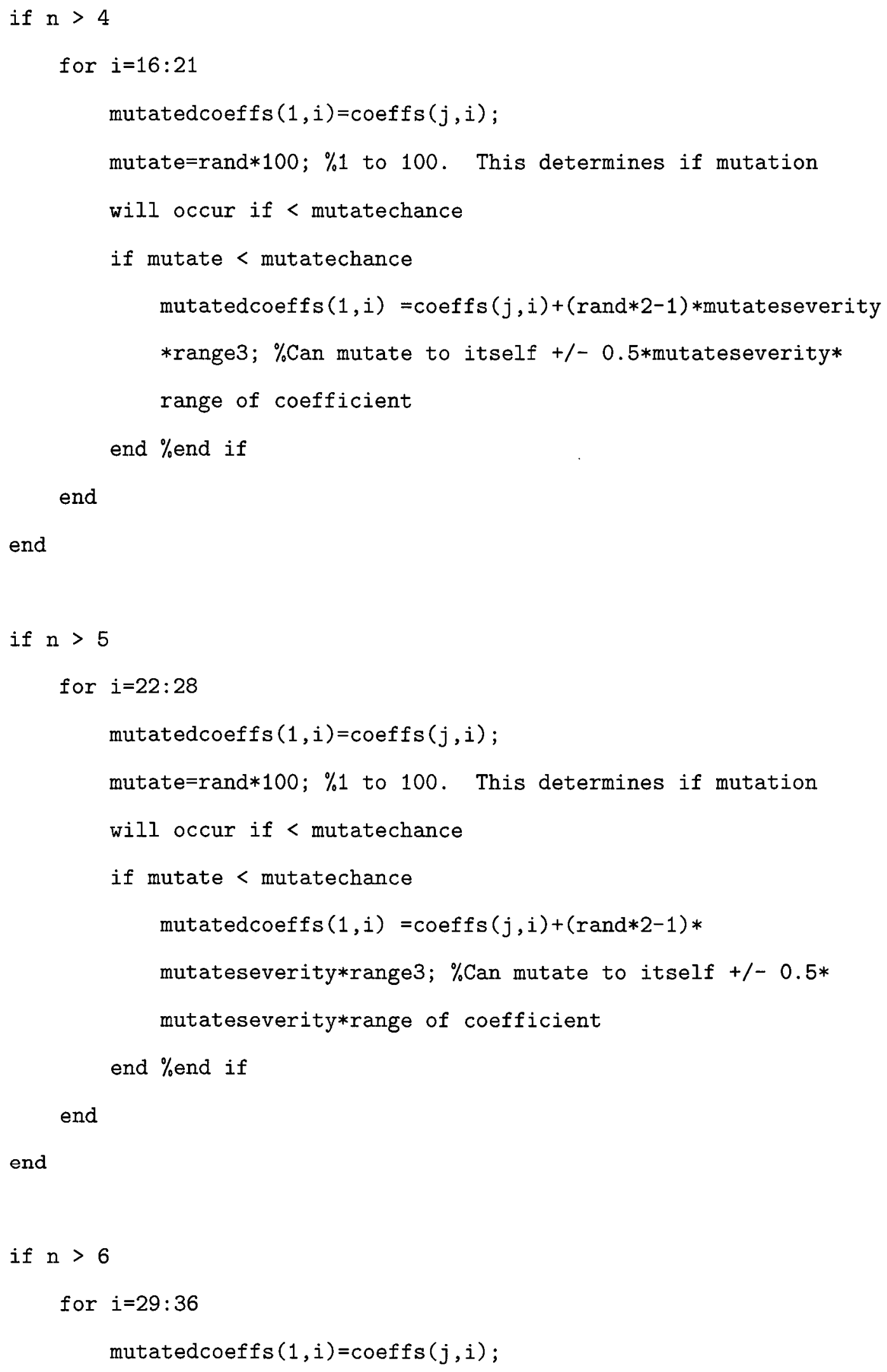


mutate $=$ rand $* 100 ; \% 1$ to 100 . This determines if mutation will occur if < mutatechance

if mutate < mutatechance $\operatorname{mutatedcoeffs}(1, i)=\operatorname{coeffs}(j, i)+(\operatorname{rand} * 2-1) *$ mutateseverity* range 3 ; Can mutate to itself $+/-0.5 *$ mutateseverity* range of coefficient

end $\%$ end if

end

end

if $n>7$

for $i=37: 45$

mutatedcoeffs $(1, i)=\operatorname{coeffs}(j, i)$;

mutate $=$ rand $* 100 ; \% 1$ to 100 . This determines if mutation

will occur if < mutatechance

if mutate < mutatechance

mutatedcoeffs $(1, i)=\operatorname{coeffs}(j, i)+(\operatorname{rand} * 2-1) *$ mutateseverity

*range 3 ; Can mutate to itself $+/-0.5$ *mutateseverity* range of coefficient

end \%end if

end

end

if $n>8$

for $i=46: 55$

mutatedcoeffs $(1, i)=\operatorname{coeffs}(j, i)$;

mutate $=$ rand $* 100 ; \% 1$ to 100 . This determines if mutation

will occur if < mutatechance

if mutate < mutatechance 
$\operatorname{mutatedcoeffs}(1, i)=\operatorname{coeffs}(j, i)+(\operatorname{rand} * 2-1) *$ mutateseverity*range $3 ; \%$ Can mutate to itself $+/-0.5 *$ mutateseverity*range of coefficient

end $\%$ end if

end

end $\%$ end if

$\%$ Evaluate function value of non-mutated

for $i=1: m$

trialvalues $(j, i)=\operatorname{coeffs}(j, 1)+(\operatorname{coeffs}(j, 2) * \operatorname{xvalues}(i))+$

$(\operatorname{coeffs}(j, 3) * \operatorname{yvalues}(i))+(\operatorname{coeffs}(j, 4) * \operatorname{xvalues}(i) \wedge 2)+(\operatorname{coeffs}(j, 5)$

*xvalues $(i) * \operatorname{yvalues}(i))+(\operatorname{coeffs}(j, 6) * \operatorname{yvalues}(i) \wedge 2)+(\operatorname{coeffs}(j, 7)$

$\left.* \operatorname{xvalues}(i)^{\wedge} 3\right)+\left(\operatorname{coeffs}(j, 8) * \operatorname{xvalues}(i)^{\wedge} 2 * \operatorname{yvalues}(i)\right)+(\operatorname{coeffs}(j, 9)$

*xvalues $\left.(i) * y v a l u e s(i)^{\sim} 2\right)+(\operatorname{coeffs}(j, 10) * \operatorname{yvalues}(i) \wedge 3)+(\operatorname{coeffs}(j, 11)$

$* x v a l u e s(i) \leadsto 4)+(\operatorname{coeffs}(j, 12) * \operatorname{xvalues}(i) \wedge 3 * \operatorname{yvalues}(i))+(\operatorname{coeffs}(j, 13)$

$* x v a l u e s(i) ` 2 * y v a l u e s(i) ` 2)+(\operatorname{coeffs}(j, 14) * \operatorname{xvalues}(i) * \operatorname{yvalues}(i) \multimap 3)$

$+\left(\operatorname{coeffs}(j, 15) * \operatorname{yvalues}(i)^{-} 4\right)+\left(\operatorname{coeffs}(j, 16) * \operatorname{xvalues}(i)^{\wedge} 5\right)+$

$(\operatorname{coeffs}(j, 17) * x v a l u e s(i)-4 * \operatorname{yvalues}(i))+(\operatorname{coeffs}(j, 18) * x v a l u e s(i) \leadsto 3 *$

yvalues $\left.(i)^{\wedge} 2\right)+\left(\operatorname{coeffs}(j, 19) * \operatorname{xvalues}(i)^{\wedge} 2 * \operatorname{yvalues}(i)^{\wedge} 3\right)+(\operatorname{coeffs}(j, 20)$

*xvalues $(i) * y v a l u e s(i)-4)+(\operatorname{coeffs}(j, 21) * \operatorname{yvalues}(i)-5)+(\operatorname{coeffs}(j, 22)$

$*$ xvalues $\left.(i)^{\wedge} 6\right)+\left(\operatorname{coeffs}(j, 23) * \operatorname{xvalues}(i)^{\wedge} 5 * \operatorname{yvalues}(i)\right)+(\operatorname{coeffs}(j, 24)$

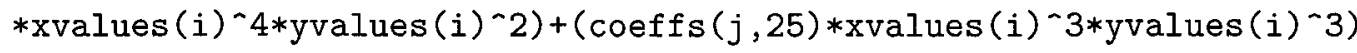

$+(\operatorname{coeffs}(j, 26) * x v a l u e s(i) \sim 2 * \operatorname{yvalues}(i) \backsim 4)+(\operatorname{coeffs}(j, 27) * \operatorname{xvalues}(i)$

*yvalues $\left.(i)^{\wedge} 5\right)+\left(\operatorname{coeffs}(j, 28) * \operatorname{yvalues}(i)^{\sim} 6\right)+(\operatorname{coeffs}(j, 29) *$

$\left.\operatorname{xvalues}(i)^{\sim} 7\right)+(\operatorname{coeffs}(j, 30) * \operatorname{xvalues}(i) \sim 6 * \operatorname{yvalues}(i))+(\operatorname{coeffs}(j, 31)$

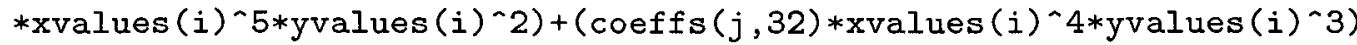

$+\left(\operatorname{coeffs}(j, 33) * \operatorname{xvalues}(i) \wedge 3 * \operatorname{yvalues}(i)^{\wedge} 4\right)+\left(\operatorname{coeffs}(j, 34) * \operatorname{xvalues}(i)^{\wedge} 2\right.$

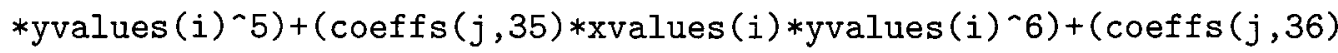

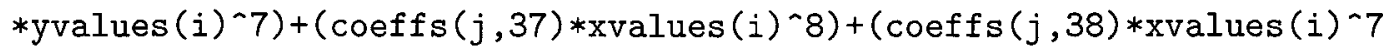

$*$ yvalues $(i))+(\operatorname{coeffs}(j, 39) * \operatorname{xvalues}(i) \wedge 6 * \operatorname{yvalues}(i) \wedge 2)+(\operatorname{coeffs}(j, 40)$

Reproduced with permission of the copyright owner. Further reproduction prohibited without permission. 
$\left.* \operatorname{xvalues}(i)^{\wedge} 5 * \operatorname{yvalues}(i)^{\wedge} 3\right)+\left(\operatorname{coeffs}(j, 41) * \operatorname{xvalues}(i)^{\wedge} 4 * \operatorname{yvalues}(i)^{\wedge} 4\right)$ $+(\operatorname{coeffs}(j, 42) * \operatorname{xvalues}(i) \wedge 3 * \operatorname{yvalues}(i) \wedge 5)+\left(\operatorname{coeffs}(j, 43) * \operatorname{xvalues}(i)^{\wedge} 2\right.$ *yvalues $\left.(i)^{\wedge} 6\right)+\left(\operatorname{coeffs}(j, 44) * \operatorname{xvalues}(i) * \operatorname{yvalues}(i)^{\wedge} 7\right)+(\operatorname{coeffs}(j, 45)$ *yvalues $\left.(i)^{\wedge}-8\right)+(\operatorname{coeffs}(j, 46) * x v a l u e s(i) ` 9)+(\operatorname{coeffs}(j, 47) * x v a l u e s(i) \wedge 8$ *yvalues $(i))+(\operatorname{coeffs}(j, 48) * \operatorname{xvalues}(i)-7 * \operatorname{yvalues}(i) \wedge 2)+(\operatorname{coeffs}(j, 49)$

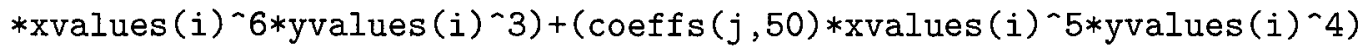
$+\left(\operatorname{coeffs}(j, 51) * \operatorname{xvalues}(i)^{\wedge} 4 * \operatorname{yvalues}(i)^{\wedge} 5\right)+(\operatorname{coeffs}(j, 52) * \operatorname{xvalues}(i) \wedge 3$ $\left.* \operatorname{yvalues}(i)^{\wedge} 6\right)+\left(\operatorname{coeffs}(j, 53) * \operatorname{xvalues}(i)^{\wedge} 2 * \operatorname{yvalues}(i)^{\wedge} 7\right)+(\operatorname{coeffs}(j, 54)$ *xvalues $(i) * y v a l u e s(i) \wedge 8)+\left(\operatorname{coeffs}(j, 55) * \operatorname{yvalues}(i)^{\wedge} 9\right)$;

end

$\%$ Evalute function value of mutated

for $i=1: m$

mutatedvalues $(1, i)=$ mutatedcoeffs $(1,1)+($ mutatedcoeffs $(1,2) * \operatorname{xvalues}(i))$

$+($ mutatedcoeffs $\left.(1,3) * \operatorname{yvalues}(i))+(\text { mutatedcoeffs }(1,4) * \operatorname{xvalues}(i))^{\prime}\right)+$

(mutatedcoeffs $(1,5) * x v a l u e s(i) * y v a l u e s(i))+($ mutatedcoeffs $(1,6) *$ yvalues $\left.(i)^{\wedge} 2\right)+($ mutatedcoeffs $(1,7) * \operatorname{xvalues}(i) \wedge 3)+($ mutatedcoeffs $(1,8)$ *xvalues (i) ^2*yvalues (i))+(mutatedcoeffs $(1,9) * x v a l u e s(i) * y v a l u e s(i)$-2)

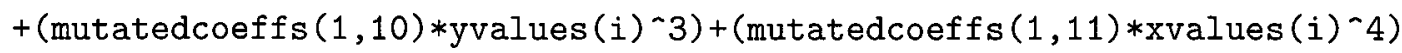
$+($ mutatedcoeffs $(1,12) * \operatorname{xvalues}(i)-3 * \operatorname{yvalues}(i))+($ mutatedcoeffs $(1,13) *$ xvalues (i)^2*yvalues (i)^2)+(mutatedcoeffs $(1,14) * x v a l u e s(i) * y v a l u e s(i) ` 3)$ $+\left(\right.$ mutatedcoeffs $(1,15) *$ yvalues $\left.(i)^{-4} 4\right)+\left(\right.$ mutatedcoeffs $\left.(1,16) * \operatorname{xvalues}(i)^{-5}\right)+$ (mutatedcoeffs $(1,17) * x v a l u e s(i) \wedge 4 * \operatorname{yvalues}(i))+($ mutatedcoeffs $(1,18) *$ xvalues (i)`3*yvalues (i)^2)+(mutatedcoeffs $(1,19) * \operatorname{xvalues}(i) ` 2 *$ yvalues (i) 3$)+($ mutatedcoeffs $(1,20) * \operatorname{xvalues}(i) * \operatorname{yvalues}(i) \sim 4)+$ (mutatedcoeffs $(1,21) * y v a l u e s(i)-5)+($ mutatedcoeffs $(1,22) * x v a l u e s(i) \sim 6)$ $+($ mutatedcoeffs $(1,23) * x v a l u e s(i) \sim 5 *$ yvalues $(i))+($ mutatedcoeffs $(1,24)$ *xvalues (i )^4*yvalues (i )^2)+(mutatedcoeffs $(1,25) * \operatorname{xvalues}(i) ` 3 *$ yvalues $\left.(i)^{\wedge} 3\right)+($ mutatedcoeffs $(1,26) * \operatorname{xvalues}(i) \wedge 2 * \operatorname{yvalues}(i) \wedge 4)+$ (mutatedcoeffs $(1,27) * \operatorname{xvalues}(i) * \operatorname{yvalues}(i) \wedge 5)+($ mutatedcoeffs $(1,28)$ 
*yvalues $(i) \sim 6)+($ mutatedcoeffs $(1,29) * \operatorname{xvalues}(i) \sim 7)+($ mutatedcoeffs $(1,30)$ *xvalues (i) - 6 *yvalues (i)) +(mutatedcoeffs $(1,31) * x v a l u e s(i) ` 5 *$ yvalues (i) ^2)+(mutatedcoeffs (1,32)*xvalues (i)^4*yvalues (i)^3)+ (mutatedcoeffs $(1,33) * x v a l u e s(i) \wedge 3 * y v a l u e s(i) \wedge 4)+($ mutatedcoeffs $(1,34)$ *xvalues (i )^2*yvalues (i )^5)+(mutatedcoeffs $(1,35) * \operatorname{xvalues}(i) *$ yvalues (i) 6$)+\left(\right.$ mutatedcoeffs $\left.(1,36) * \operatorname{yvalues}(i)^{\sim} 7\right)+($ mutatedcoeffs $(1,37)$ *xvalues (i ) ^ 8$)+\left(\right.$ mutatedcoeffs $\left.(1,38) * \operatorname{xvalues}(i)^{\wedge} 7 * \operatorname{yvalues}(i)\right)+$ (mutatedcoeffs $(1,39) * \operatorname{xvalues}(i)^{\sim} 6 *$ yvalues (i) 2$)+($ mutatedcoeffs $(1,40)$ *xvalues (i )`5*yvalues (i)^3)+(mutatedcoeffs $(1,41) * \operatorname{xvalues}(i) \sim 4 *$ yvalues (i) ^4)+(mutatedcoeffs $(1,42) * \operatorname{xvalues}(i)^{\wedge} 3 *$ yvalues (i)^5)+ (mutatedcoeffs $(1,43) * x v a l u e s(i) ` 2 * y v a l u e s(i) \wedge 6)+($ mutatedcoeffs $(1,44)$ *xvalues (i) *yvalues (i) $\left.{ }^{\sim} 7\right)+\left(\right.$ mutatedcoeffs $\left.(1,45) * \operatorname{yvalues}(i)^{\sim} 8\right)+$ (mutatedcoeffs $\left.(1,46) * \operatorname{xvalues}(i)^{\wedge} 9\right)+($ mutatedcoeffs $(1,47) * \operatorname{xvalues}(i) \wedge 8 *$ yvalues (i)) +(mutatedcoeffs $(1,48) * \operatorname{xvalues}(i) \wedge 7 * \operatorname{yvalues}(i) \wedge 2)+$ (mutatedcoeffs $(1,49) * \operatorname{xvalues}(i) \wedge 6 * \operatorname{yvalues}(i) ` 3)+($ mutatedcoeffs $(1,50) *$ xvalues (i )`5*yvalues (i)^4)+(mutatedcoeffs $(1,51) * \operatorname{xvalues}(i) \wedge 4 *$ yvalues(i) ^5)+(mutatedcoeffs $\left.(1,52) * \operatorname{xvalues}(i)^{\wedge} 3 * \operatorname{yvalues}(i)^{\wedge} 6\right)+$ (mutatedcoeffs $\left.(1,53) * \operatorname{xvalues}(i)^{\sim} 2 * \operatorname{yvalues}(i)^{\sim} 7\right)+($ mutatedcoeffs $(1,54) *$ xvalues (i)*yvalues (i)^8)+(mutatedcoeffs $\left.(1,55) * \operatorname{yvalues}(i)^{\wedge} 9\right)$;

end

$\%$ Now we need to find the error of the non-mutated errorsum=0; \%square root of sum of squares for $i=1: m$ errorsum $=$ errorsum $+($ trialvalues $(j, i)-$ functionvalues $(1, i)) \wedge 2$; end $\operatorname{error}(j, 1)=\operatorname{sqrt}($ errorsum $)$; 


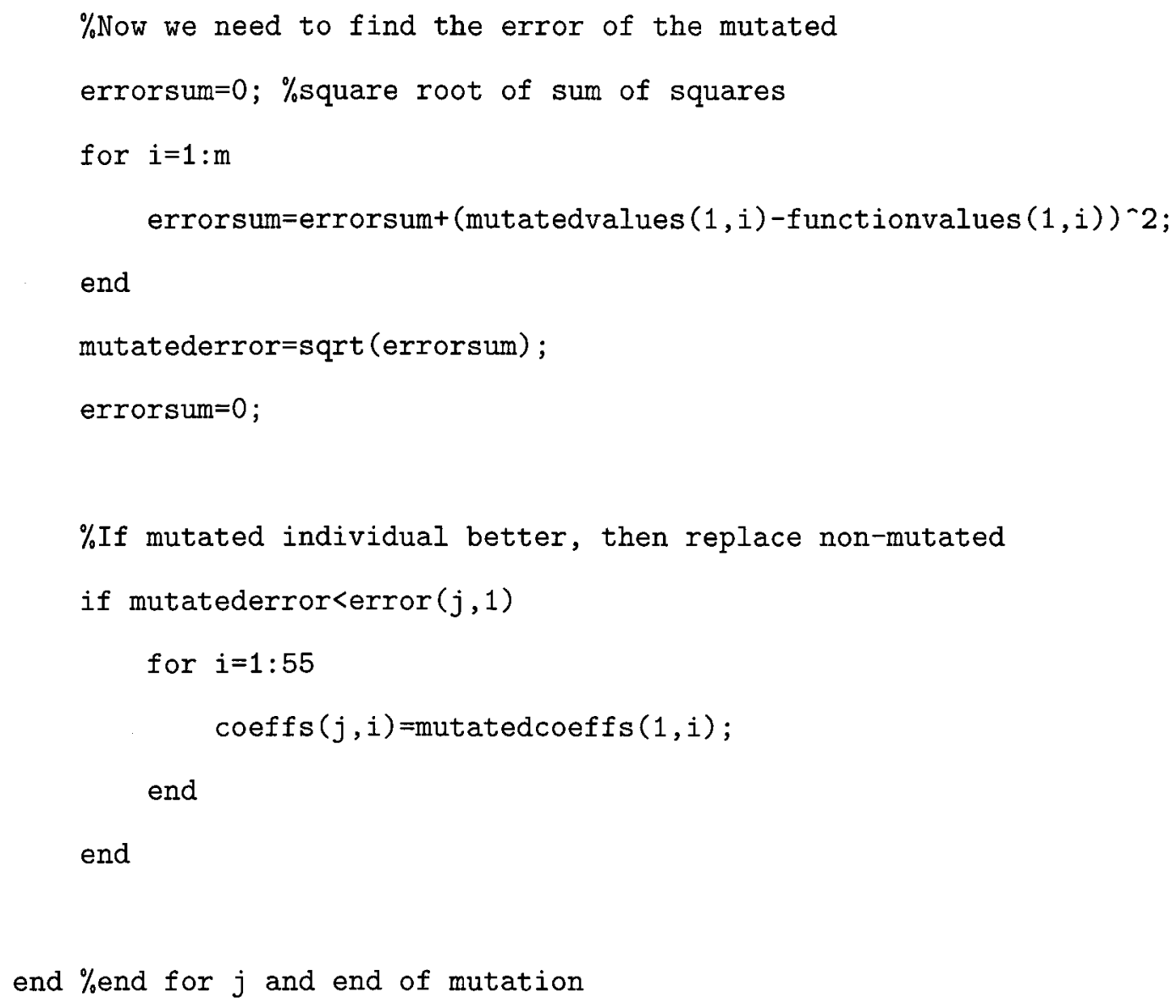

\%THE INDIVIDUALS NOT SELECTED FOR BREEDING WILL BE REPLACED BY NEW RANDOM \%INDIVIDUALS (immigration)

\%----NOW WE NEED TO RE-EVALUATE FITNESS AND RE-SELECT.

\%determine fitness: square root of sum of squared errors $\%$ first we need to re-evaluate the function value

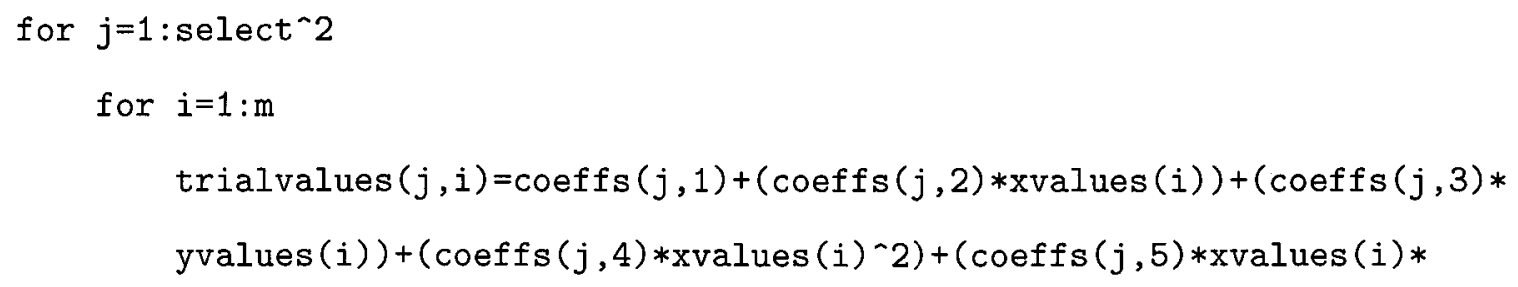


yvalues $(i))+\left(\operatorname{coeffs}(j, 6) * \operatorname{yvalues}(i)^{\wedge} 2\right)+\left(\operatorname{coeffs}(j, 7) * \operatorname{xvalues}(i)^{\wedge} 3\right)+$ $(\operatorname{coeffs}(j, 8) * \operatorname{xvalues}(i) \wedge 2 * \operatorname{yvalues}(i))+(\operatorname{coeffs}(j, 9) * \operatorname{xvalues}(i) *$ yvalues $(i) \wedge 2)+(\operatorname{coeffs}(j, 10) * \operatorname{yvalues}(i) \wedge 3)+(\operatorname{coeffs}(j, 11) *$ $\operatorname{xvalues}(i) \wedge 4)+(\operatorname{coeffs}(j, 12) * \operatorname{xvalues}(i) \wedge 3 * \operatorname{yvalues}(i))+(\operatorname{coeffs}(j, 13) *$

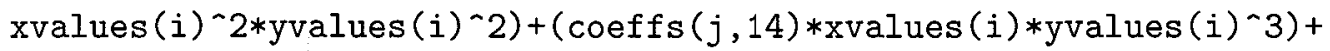
$\left(\operatorname{coeffs}(j, 15) * \operatorname{yvalues}(i)^{-4}\right)+\left(\operatorname{coeffs}(j, 16) * \operatorname{xvalues}(i)^{\wedge} 5\right)+$ $(\operatorname{coeffs}(j, 17) * x \operatorname{xalues}(i) \sim 4 * \operatorname{yvalues}(i))+\left(\operatorname{coeffs}(j, 18) * \operatorname{xvalues}(i)^{\wedge} 3 *\right.$ yvalues $\left.(i)^{\wedge} 2\right)+\left(\operatorname{coeffs}(j, 19) * \operatorname{xvalues}(i)^{\wedge} 2 * \operatorname{yvalues}(i)^{\wedge} 3\right)+$ $(\operatorname{coeffs}(j, 20) * x v a l u e s(i) * \operatorname{yvalues}(i) \sim 4)+(\operatorname{coeffs}(j, 21) * \operatorname{yvalues}(i) \sim 5)+$ $\left(\operatorname{coeffs}(j, 22) * \operatorname{xvalues}(i)^{\wedge} 6\right)+\left(\operatorname{coeffs}(j, 23) * \operatorname{xvalues}(i)^{\wedge} 5 * \operatorname{yvalues}(i)\right)+$ $\left(\operatorname{coeffs}(j, 24) * \operatorname{xvalues}(i)^{\wedge} 4 * \operatorname{yvalues}(i)^{\wedge} 2\right)+\left(\operatorname{coeffs}(j, 25) * \operatorname{xvalues}(i)^{\wedge} 3 *\right.$ yvalues $(i) \wedge 3)+(\operatorname{coeffs}(j, 26) * x \operatorname{xalues}(i) \wedge 2 * \operatorname{yvalues}(i) \wedge 4)+$ $\left(\operatorname{coeffs}(j, 27) * \operatorname{xvalues}(i) * \operatorname{yvalues}(i)^{\wedge} 5\right)+\left(\operatorname{coeffs}(j, 28) * \operatorname{yvalues}(i)^{\wedge} 6\right)+$ $\left(\operatorname{coeffs}(j, 29) * \operatorname{xvalues}(i)^{\wedge} 7\right)+\left(\operatorname{coeffs}(j, 30) * \operatorname{xvalues}(i)^{\wedge} 6 * \operatorname{yvalues}(i)\right)+$ $\left(\operatorname{coeffs}(j, 31) * \operatorname{xvalues}(i)^{\wedge} 5 * \operatorname{yvalues}(i)^{\wedge} 2\right)+\left(\operatorname{coeffs}(j, 32) * \operatorname{xvalues}(i)^{\wedge} 4 *\right.$ yvalues $(i) \wedge 3)+(\operatorname{coeffs}(j, 33) * \operatorname{xvalues}(i) \wedge 3 * \operatorname{yvalues}(i) \wedge 4)+$ $\left(\operatorname{coeffs}(j, 34) * \operatorname{xvalues}(i)^{\wedge} 2 * \operatorname{yvalues}(i)^{\wedge} 5\right)+(\operatorname{coeffs}(j, 35) * \operatorname{xvalues}(i) *$ yvalues $\left.(i)^{\wedge} 6\right)+\left(\operatorname{coeffs}(j, 36) * \operatorname{yvalues}(i)^{\wedge} 7\right)+(\operatorname{coeffs}(j, 37) *$ xvalues $\left.(i)^{\wedge} 8\right)+\left(\operatorname{coeffs}(j, 38) * \operatorname{xvalues}(i)^{\wedge} 7 * \operatorname{yvalues}(i)\right)+(\operatorname{coeffs}(j, 39) *$ xvalues (i)^ $6 *$ yvalues $\left.(i)^{\wedge} 2\right)+\left(\operatorname{coeffs}(j, 40) * \operatorname{xvalues}(i)^{\wedge} 5 *\right.$ $\operatorname{yvalues}(i) \wedge 3)+(\operatorname{coeffs}(j, 41) * \operatorname{xvalues}(i) \wedge 4 * \operatorname{yvalues}(i) \wedge 4)+(\operatorname{coeffs}(j, 42) *$

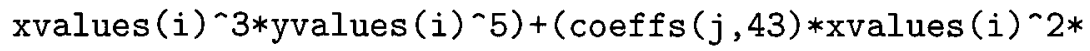
yvalues $\left.(i)^{\sim} 6\right)+(\operatorname{coeffs}(j, 44) * \operatorname{xvalues}(i) * \operatorname{yvalues}(i) \sim 7)+(\operatorname{coeffs}(j, 45) *$ yvalues $\left.(i)^{\wedge} 8\right)+\left(\operatorname{coeffs}(j, 46) * \operatorname{xvalues}(i)^{\wedge} 9\right)+(\operatorname{coeffs}(j, 47) *$ xvalues (i)^ $8 * \operatorname{yvalues}(i))+\left(\operatorname{coeffs}(j, 48) * \operatorname{xvalues}(i)^{\wedge} 7 * \operatorname{yvalues}(i) \wedge 2\right)+$ (coeffs $\left.(j, 49) * \operatorname{xvalues}(i)^{\sim} 6 * \operatorname{yvalues}(i)^{\wedge} 3\right)+(\operatorname{coeffs}(j, 50) *$

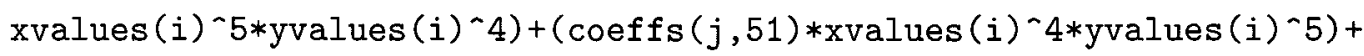
$(\operatorname{coeffs}(j, 52) * \operatorname{xvalues}(i)-3 * \operatorname{yvalues}(i) \wedge 6)+(\operatorname{coeffs}(j, 53) *$ $\operatorname{xvalues}(i) \wedge 2 * \operatorname{yvalues}(i) \wedge 7)+(\operatorname{coeffs}(j, 54) * \operatorname{xvalues}(i) * \operatorname{yvalues}(i) \wedge 8)+$ 


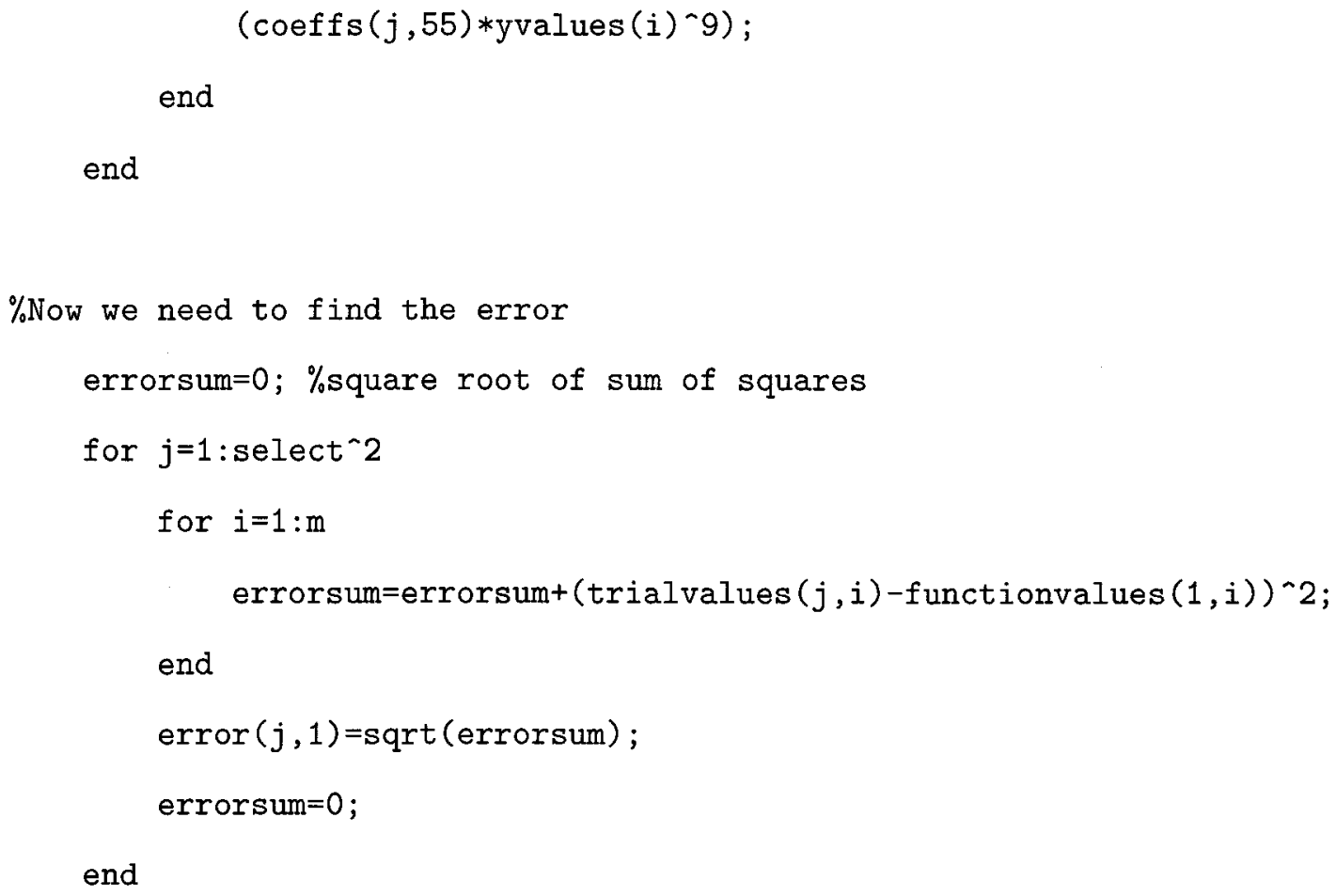

\%Replace the non-breeding indivisuals with immigrating members of the $\%$ population close to the best individual

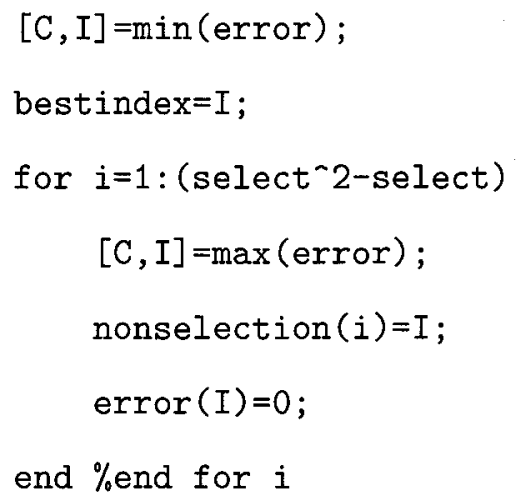




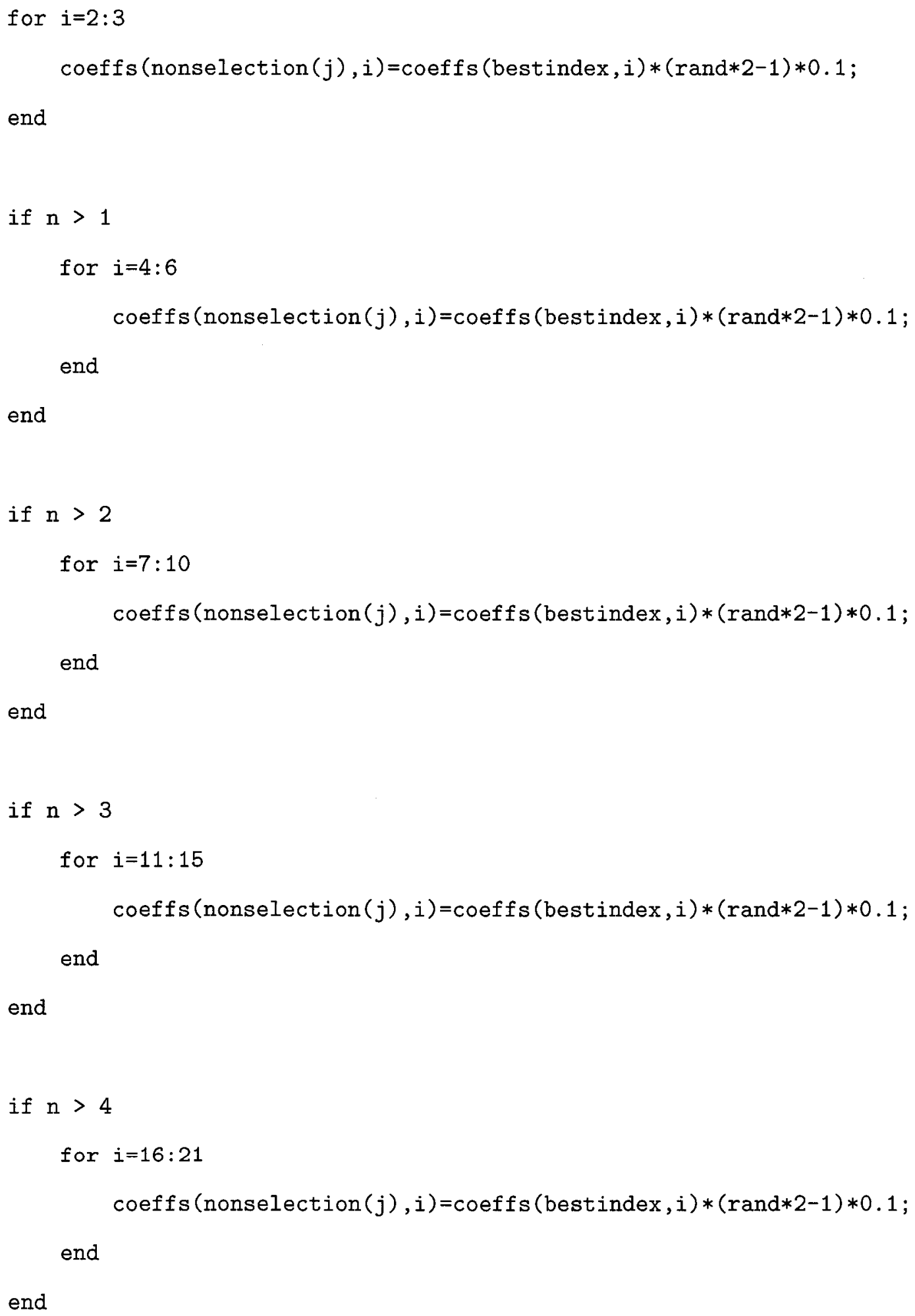




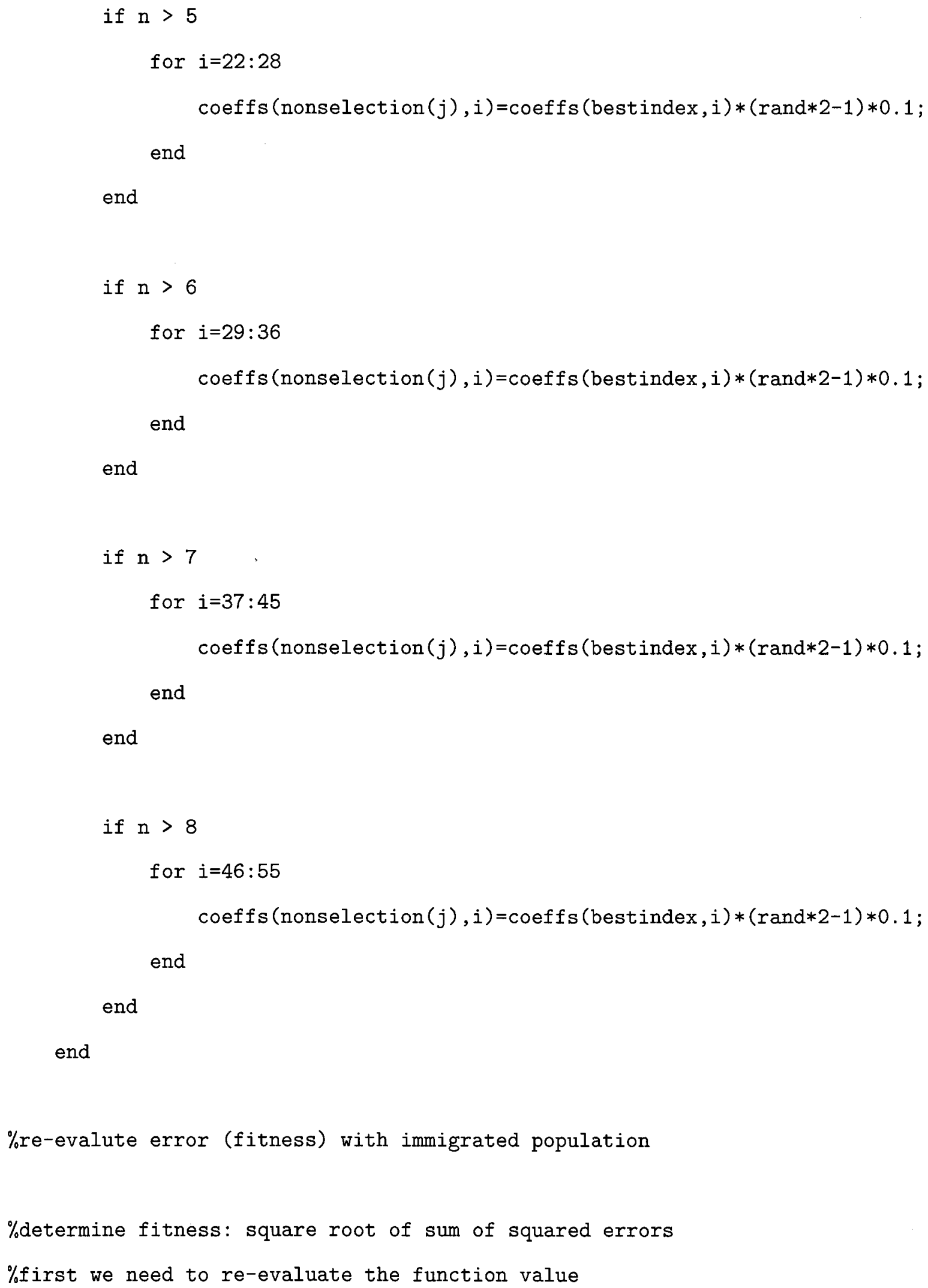




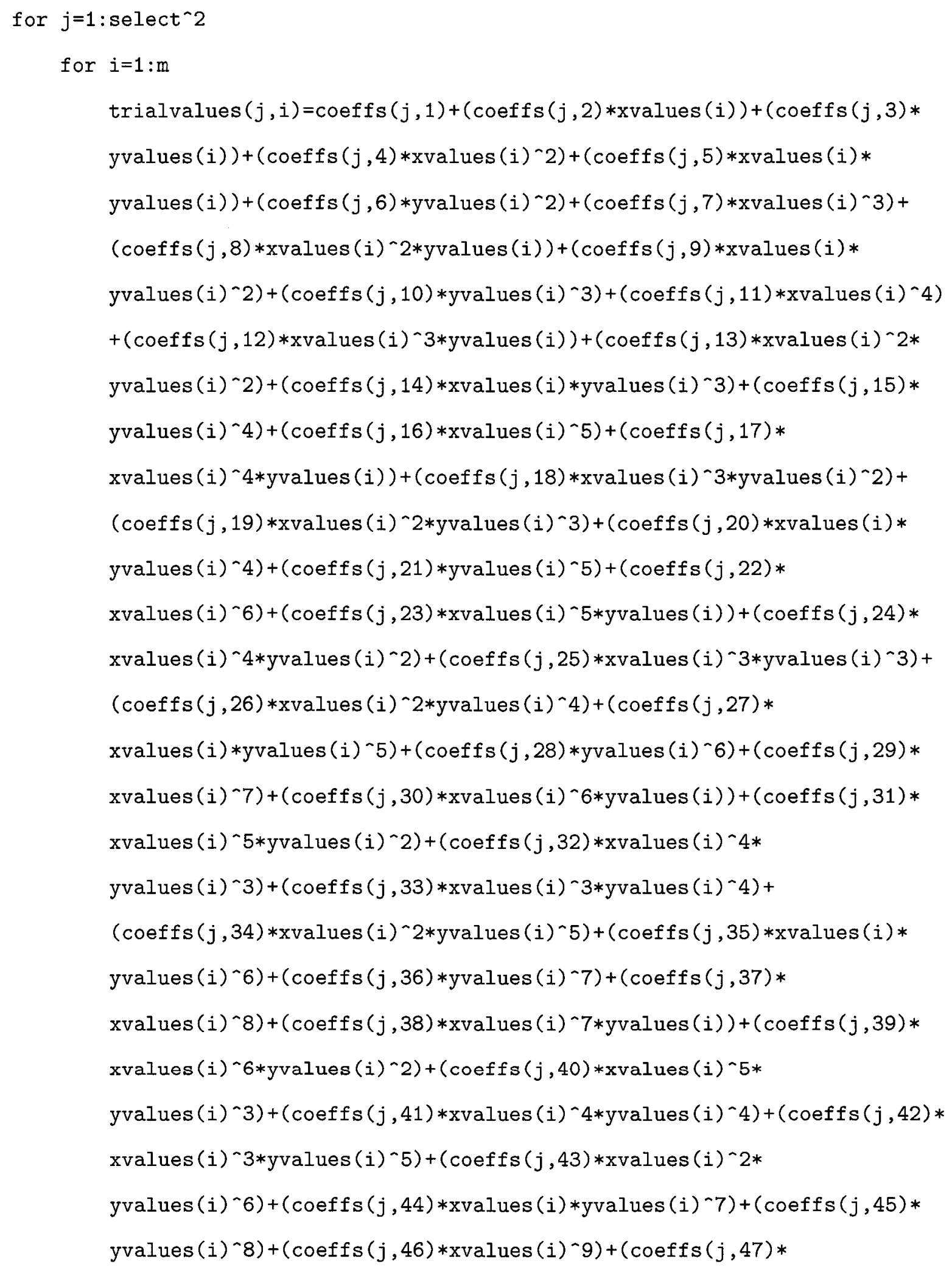




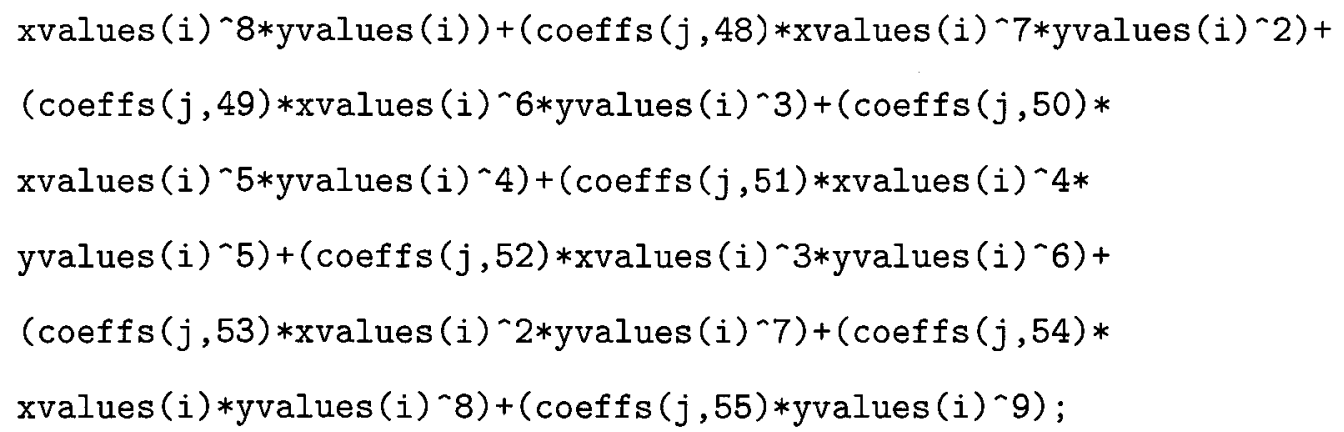


$j=$ selection(1); \%set $j$ to index of best value

$\%$ display values at $m$ points

for $i=1: m$

$\mathrm{x}=\mathrm{xvalues}(\mathrm{i})$

$y=y v a l u e s(i)$

zvalue $=\operatorname{coeffs}(j, 1)+(\operatorname{coeffs}(j, 2) * \operatorname{xvalues}(i))+(\operatorname{coeffs}(j, 3) *$

yvalues $(i))+\left(\operatorname{coeffs}(j, 4) * \operatorname{xvalues}(i)^{\wedge} 2\right)+(\operatorname{coeffs}(j, 5) * \operatorname{xvalues}(i) *$

yvalues $(i))+(\operatorname{coeffs}(j, 6) * \operatorname{yvalues}(i) \wedge 2)+(\operatorname{coeffs}(j, 7) * \operatorname{xvalues}(i) \wedge 3)+$

$\left(\operatorname{coeffs}(j, 8) * \operatorname{xvalues}(i)^{\wedge} 2 * \operatorname{yvalues}(i)\right)+(\operatorname{coeffs}(j, 9) * \operatorname{xvalues}(i) *$

yvalues $\left.(i)^{\wedge} 2\right)+(\operatorname{coeffs}(j, 10) * \operatorname{yvalues}(i) \wedge 3)+(\operatorname{coeffs}(j, 11) * \operatorname{xvalues}(i) \wedge 4)+$

(coeffs $(j, 12) * \operatorname{xvalues}(i) \wedge 3 * \operatorname{yvalues}(i))+(\operatorname{coeffs}(j, 13) * \operatorname{xvalues}(i) \wedge 2 *$

yvalues $\left.(i)^{\wedge} 2\right)+(\operatorname{coeffs}(j, 14) * \operatorname{xvalues}(i) * \operatorname{yvalues}(i) \wedge 3)+(\operatorname{coeffs}(j, 15) *$

yvalues $(i)-4)+(\operatorname{coeffs}(j, 16) * \operatorname{xvalues}(i)-5)+(\operatorname{coeffs}(j, 17) * \operatorname{xvalues}(i) \wedge 4 *$

yvalues $(i))+(\operatorname{coeffs}(j, 18) * \operatorname{xvalues}(i)-3 * \operatorname{yvalues}(i) \wedge 2)+(\operatorname{coeffs}(j, 19) *$

xvalues (i)^2*yvalues $(i) \wedge 3)+(\operatorname{coeffs}(j, 20) * \operatorname{xvalues}(i) * \operatorname{yvalues}(i) \wedge 4)+$

$(\operatorname{coeffs}(j, 21) * \operatorname{yvalues}(i)-5)+(\operatorname{coeffs}(j, 22) * \operatorname{xvalues}(i) \wedge 6)+(\operatorname{coeffs}(j, 23) *$

$\operatorname{xvalues}(i) ` 5 * \operatorname{yvalues}(i))+(\operatorname{coeffs}(j, 24) * \operatorname{xvalues}(i) \wedge 4 * \operatorname{yvalues}(i) \wedge 2)+$

(coeffs $\left.(j, 25) * x v a l u e s(i)^{\wedge} 3 * \operatorname{yvalues}(i)^{\wedge} 3\right)+\left(\operatorname{coeffs}(j, 26) * x v a l u e s(i)^{\wedge} 2 *\right.$

yvalues (i) 4$)+\left(\operatorname{coeffs}(j, 27) * \operatorname{xvalues}(i) * \operatorname{yvalues}(i)^{\sim} 5\right)+(\operatorname{coeffs}(j, 28) *$

yvalues $\left.(i)^{\wedge} 6\right)+\left(\operatorname{coeffs}(j, 29) * \operatorname{xvalues}(i)^{\wedge} 7\right)+\left(\operatorname{coeffs}(j, 30) * \operatorname{xvalues}(i)^{\wedge} 6 *\right.$

yvalues $(i))+(\operatorname{coeffs}(j, 31) * \operatorname{xvalues}(i) \wedge 5 * \operatorname{yvalues}(i) \wedge 2)+(\operatorname{coeffs}(j, 32) *$

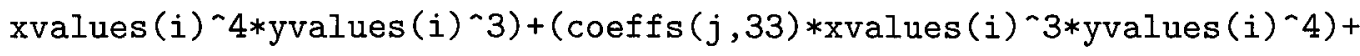

$(\operatorname{coeffs}(j, 34) * \operatorname{xvalues}(i) \wedge 2 * \operatorname{yvalues}(i) \sim 5)+(\operatorname{coeffs}(j, 35) * \operatorname{xvalues}(i) *$

yvalues $\left.(i)^{\wedge} 6\right)+(\operatorname{coeffs}(j, 36) * \operatorname{yvalues}(i) \wedge 7)+(\operatorname{coeffs}(j, 37) * \operatorname{xvalues}(i) \wedge 8)+$

$(\operatorname{coeffs}(j, 38) * \operatorname{xvalues}(i) \wedge 7 * \operatorname{yvalues}(i))+(\operatorname{coeffs}(j, 39) * \operatorname{xvalues}(i) \wedge 6 *$

yvalues $\left.(i)^{\wedge} 2\right)+(\operatorname{coeffs}(j, 40) * \operatorname{xvalues}(i) \wedge 5 * \operatorname{yvalues}(i)-3)+(\operatorname{coeffs}(j, 41) *$

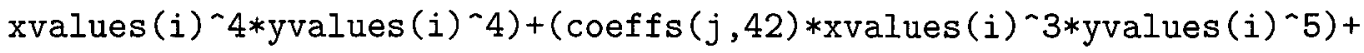




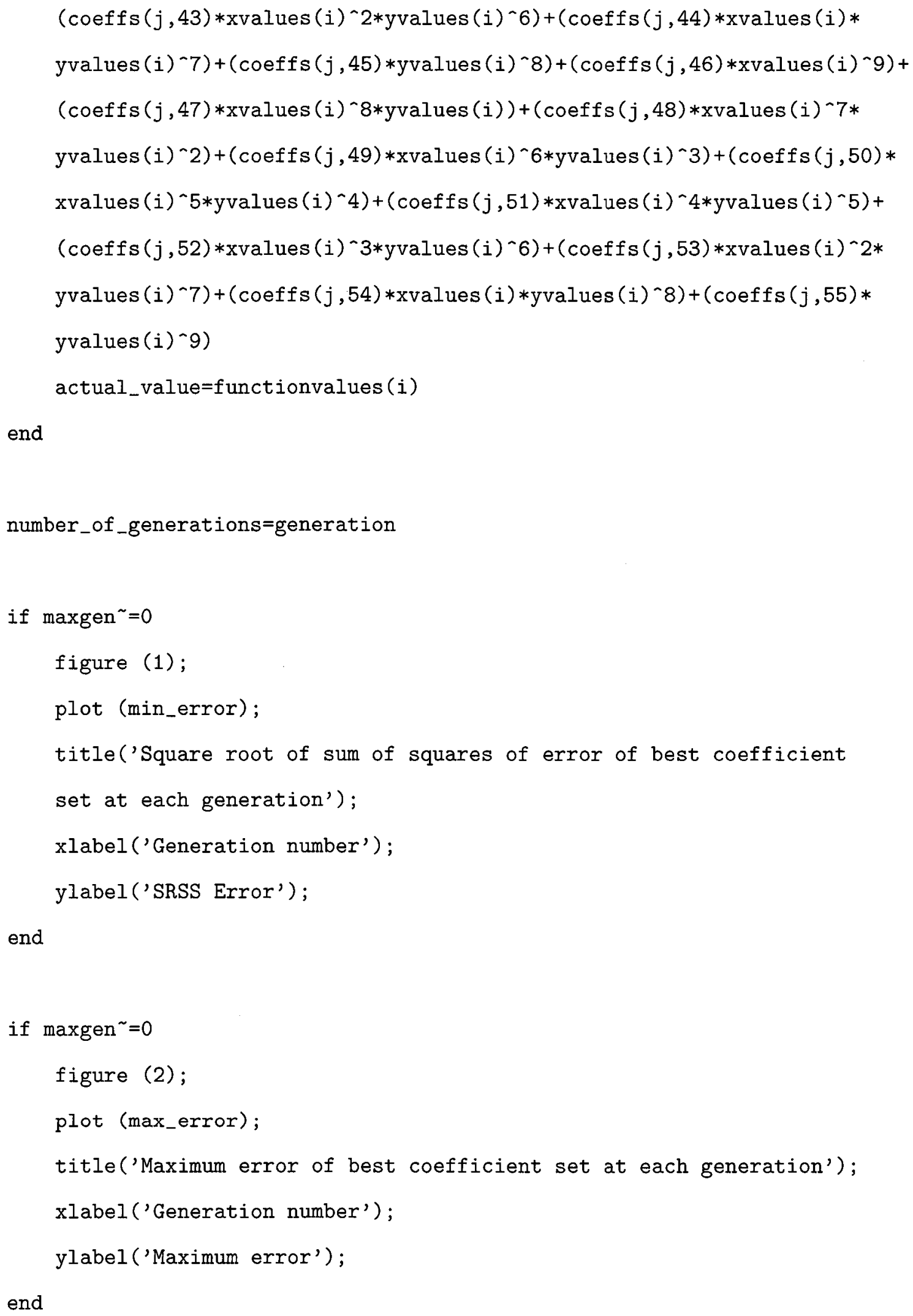




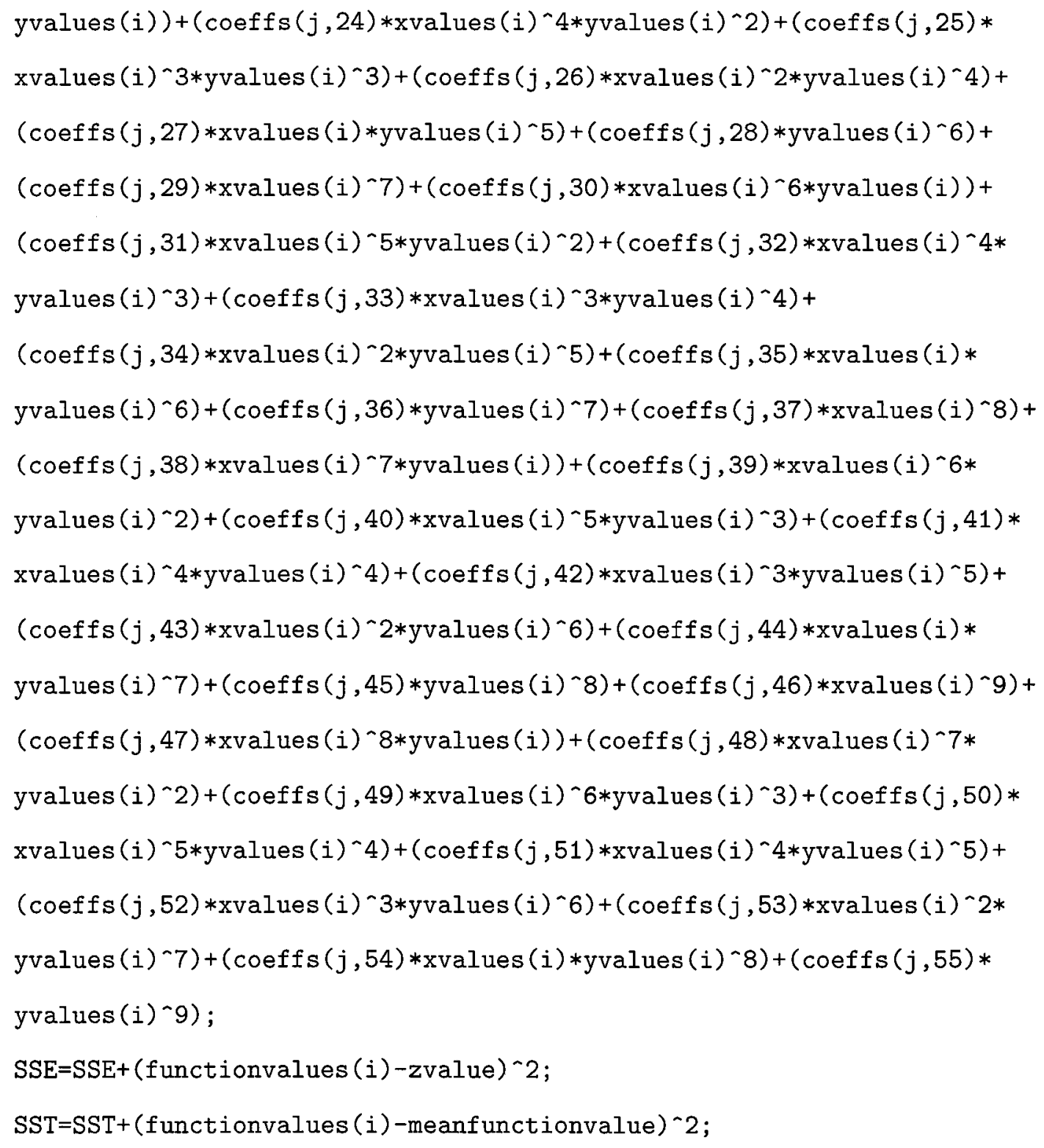




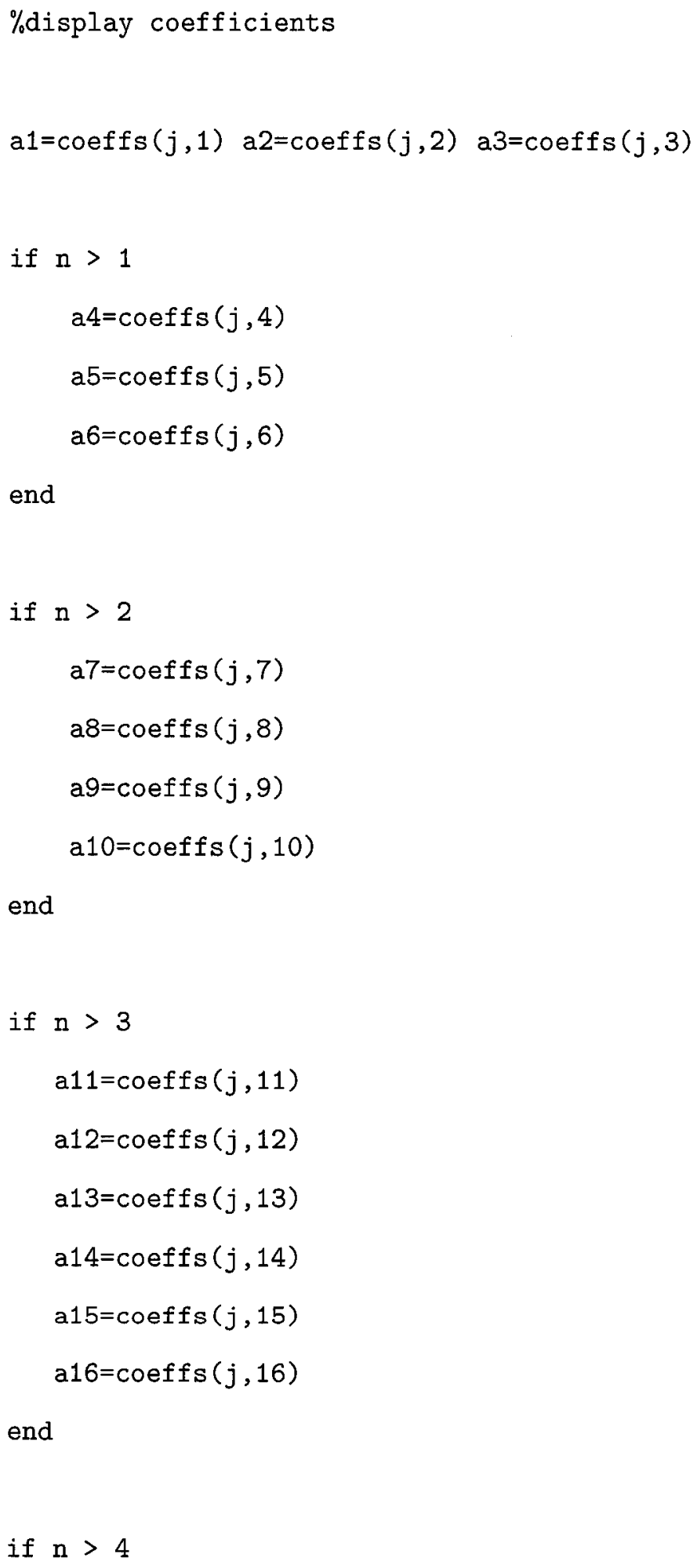




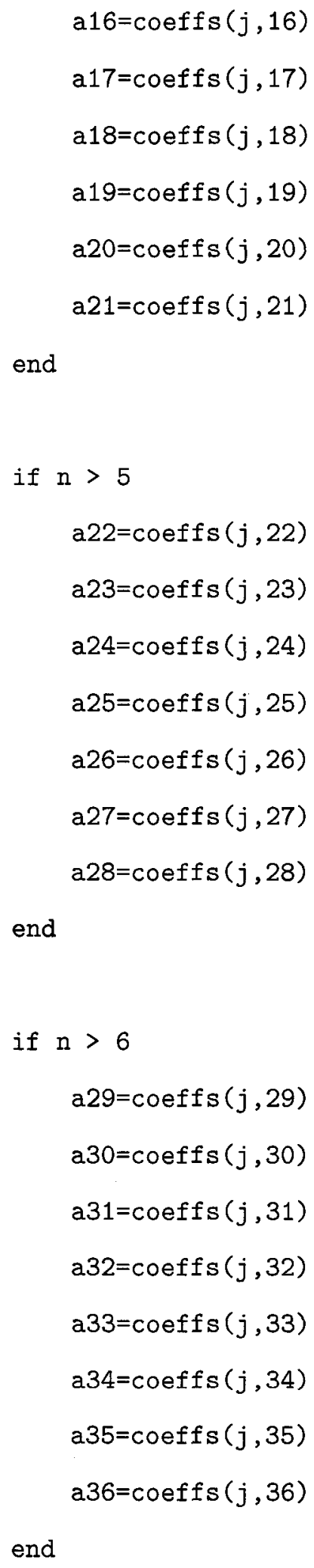




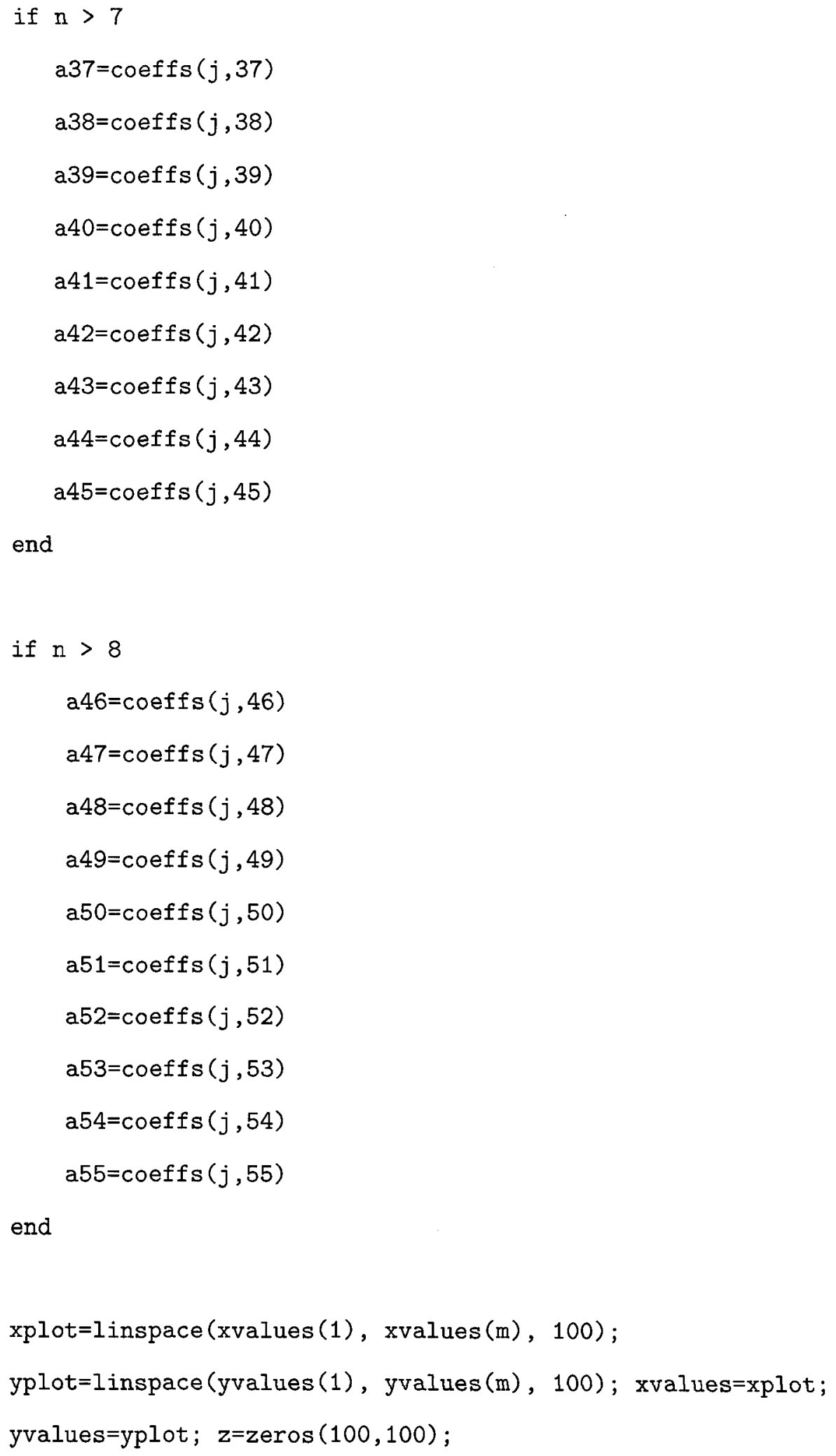


for $r=1: 100 ; \%$ values

for $i=1: 100 ; \%$ values

$$
\begin{aligned}
& z(r, i)=\operatorname{coeffs}(j, 1)+(\operatorname{coeffs}(j, 2) * \operatorname{xvalues}(r))+(\operatorname{coeffs}(j, 3) * \\
& \text { yvalues }(i))+\left(\operatorname{coeffs}(j, 4) * \operatorname{xvalues}(r)^{\wedge} 2\right)+(\operatorname{coeffs}(j, 5) * \operatorname{xvalues}(r) * \\
& \text { yvalues }(i))+\left(\operatorname{coeffs}(j, 6) * \operatorname{yvalues}(i)^{\wedge} 2\right)+(\operatorname{coeffs}(j, 7) * \\
& \text { xvalues } \left.(r)^{\wedge} 3\right)+\left(\operatorname{coeffs}(j, 8) * \operatorname{xvalues}(r)^{\wedge} 2 * \operatorname{yvalues}(i)\right)+ \\
& (\operatorname{coeffs}(j, 9) * x v a l u e s(r) * \operatorname{yvalues}(i) \sim 2)+(\operatorname{coeffs}(j, 10) * \\
& \text { yvalues } \left.(i)^{\wedge} 3\right)+\left(\operatorname{coeffs}(j, 11) * x \operatorname{xvalues}(r)^{\wedge} 4\right)+(\operatorname{coeffs}(j, 12) * \\
& \left.\operatorname{xvalues}(r)^{\wedge} 3 * \text { yvalues }(i)\right)+\left(\operatorname{coeffs}(j, 13) * \operatorname{xvalues}(r)^{\wedge} 2 *\right. \\
& \text { yvalues }(i) \wedge 2)+(\operatorname{coeffs}(j, 14) * x v a l u e s(r) * y v a l u e s(i) ` 3)+ \\
& (\operatorname{coeffs}(j, 15) * \operatorname{yvalues}(i) \wedge 4)+\left(\operatorname{coeffs}(j, 16) * \operatorname{xvalues}(r)^{\wedge} 5\right)+ \\
& (\operatorname{coeffs}(j, 17) * x v a l u e s(r) \leadsto 4 * \operatorname{yvalues}(i))+(\operatorname{coeffs}(j, 18) * \\
& \operatorname{xvalues}(r) \text { ^3*yvalues }(i) \frown 2)+\left(\operatorname{coeffs}(j, 19) * \operatorname{xvalues}(r)^{\wedge} 2 *\right. \\
& \text { yvalues }(i) \wedge 3)+(\operatorname{coeffs}(j, 20) * \operatorname{xvalues}(r) * y v a l u e s(i) \wedge 4)+ \\
& \left(\operatorname{coeffs}(j, 21) * \operatorname{yvalues}(i)^{\wedge} 5\right)+\left(\operatorname{coeffs}(j, 22) * \operatorname{xvalues}(r)^{\wedge} 6\right)+ \\
& \left(\operatorname{coeffs}(j, 23) * \operatorname{xvalues}(r)^{\sim} 5 * \operatorname{yvalues}(i)\right)+(\operatorname{coeffs}(j, 24) *
\end{aligned}
$$

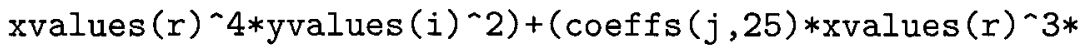

$$
\begin{aligned}
& \text { yvalues }(i) \wedge 3)+\left(\operatorname{coeffs}(j, 26) * \operatorname{xvalues}(r)^{\wedge} 2 * \operatorname{yvalues}(i)^{\wedge} 4\right)+ \\
& \left(\operatorname{coeffs}(j, 27) * \operatorname{xvalues}(r) * \operatorname{yvalues}(i)^{\wedge} 5\right)+(\operatorname{coeffs}(j, 28) * \\
& \text { yvalues } \left.(i)^{\wedge} 6\right)+\left(\operatorname{coeffs}(j, 29) * \operatorname{xvalues}(r)^{\wedge} 7\right)+(\operatorname{coeffs}(j, 30) * \\
& \left.\operatorname{xvalues}(r)^{\sim} 6 * \operatorname{yvalues}(i)\right)+\left(\operatorname{coeffs}(j, 31) * \operatorname{xvalues}(r)^{\wedge} 5 *\right. \\
& \text { yvalues } \left.(i)^{\wedge} 2\right)+\left(\operatorname{coeffs}(j, 32) * \operatorname{xvalues}(r)^{\wedge} 4 * \operatorname{yvalues}(i) \wedge 3\right)+ \\
& \left(\operatorname{coeffs}(j, 33) * x v a l u e s(r)^{\wedge} 3 * \operatorname{yvalues}(i)^{\wedge} 4\right)+(\operatorname{coeffs}(j, 34) * \\
& \left.\operatorname{xvalues}(r)^{\wedge} 2 * \text { yvalues }(i)^{\wedge} 5\right)+(\operatorname{coeffs}(j, 35) * \operatorname{xvalues}(r) * \\
& \text { yvalues } \left.(i)^{\wedge} 6\right)+\left(\operatorname{coeffs}(j, 36) * \operatorname{yvalues}(i)^{\wedge} 7\right)+(\operatorname{coeffs}(j, 37) * \\
& \left.\operatorname{xvalues}(r)^{\wedge} 8\right)+\left(\operatorname{coeffs}(j, 38) * \operatorname{xvalues}(r)^{\wedge} 7 * \operatorname{yvalues}(i)\right)+ \\
& \left(\operatorname{coeffs}(j, 39) * \operatorname{xvalues}(r) \leadsto 6 * \operatorname{yvalues}(i)^{\wedge} 2\right)+(\operatorname{coeffs}(j, 40) * \\
& \text { xvalues }(r) \wedge 5 * \text { yvalues }(i) \wedge 3)+\left(\operatorname{coeffs}(j, 41) * \operatorname{xvalues}(r)^{\wedge} 4 *\right.
\end{aligned}
$$




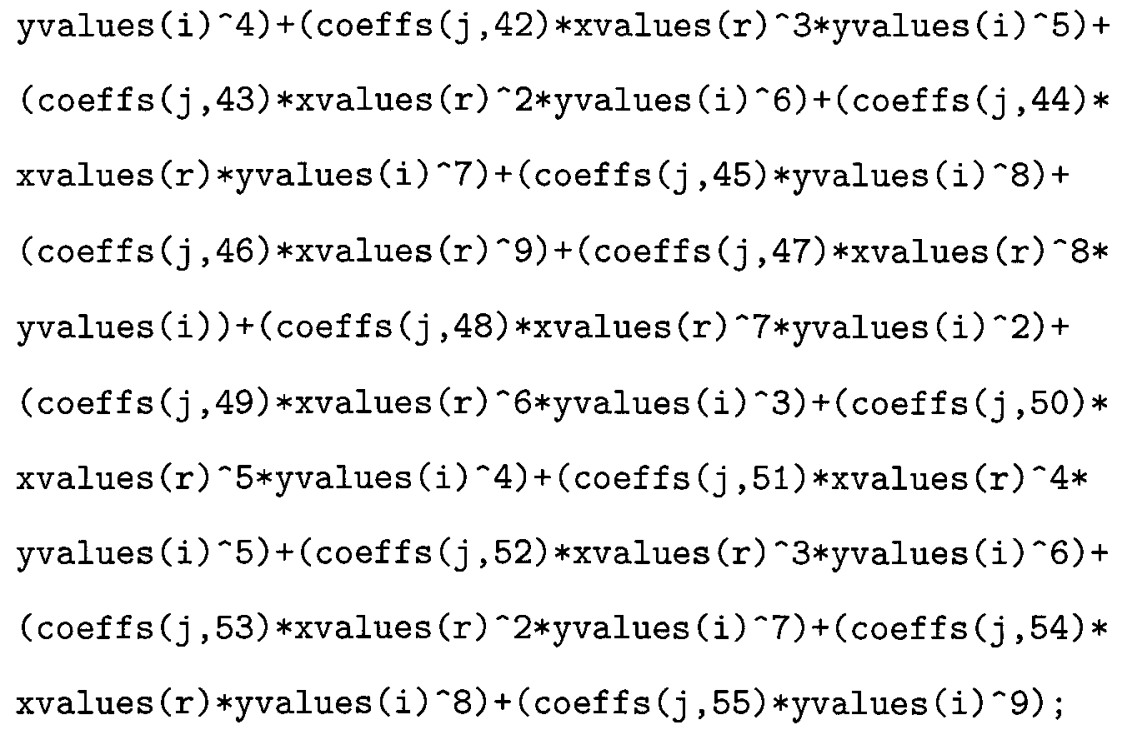




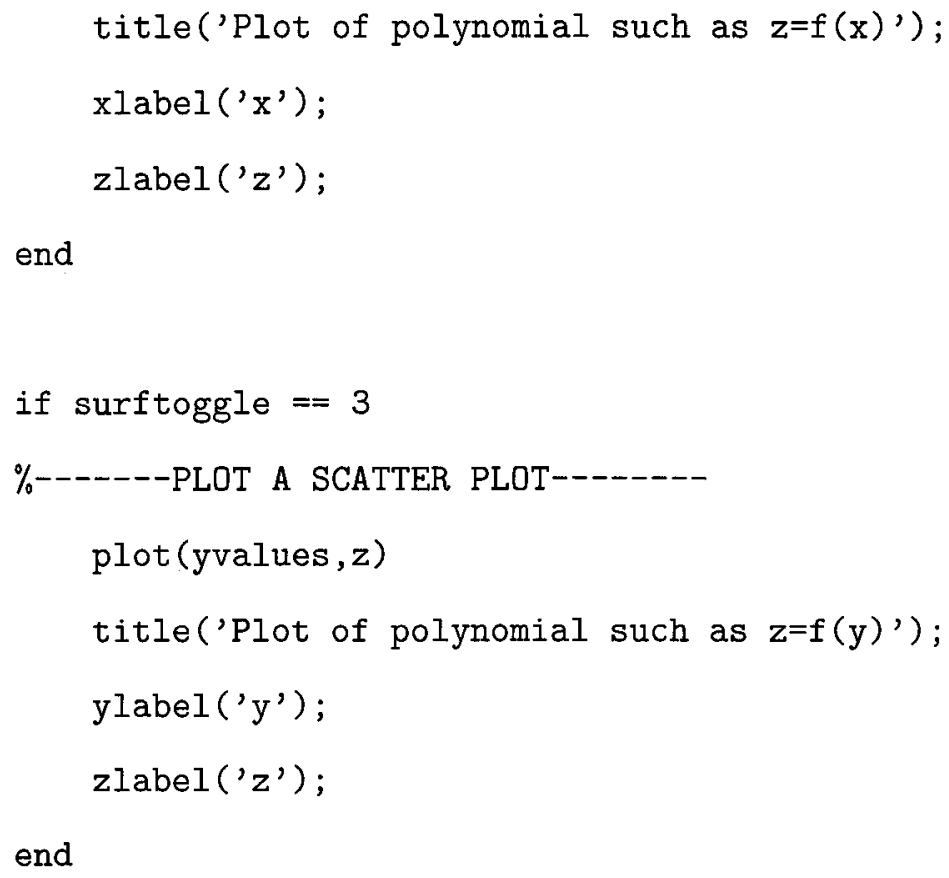


ylabel ('Maximum error $\left.(\%)^{\prime}\right)$;

end

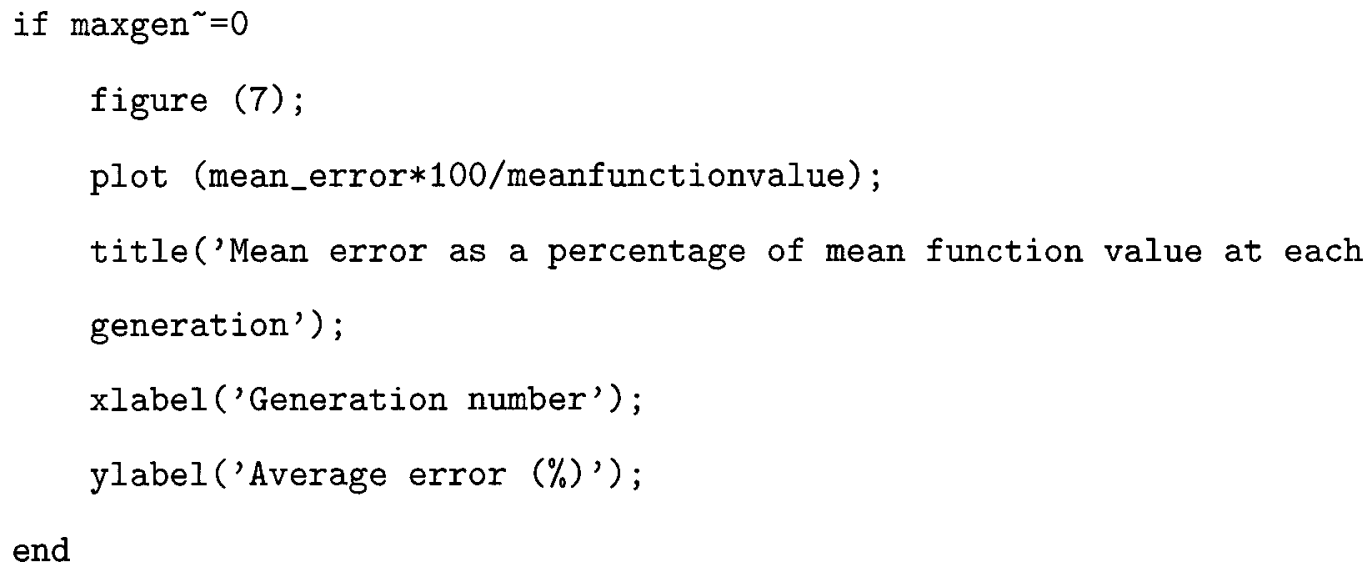




\section{Appendix B}

\section{Single Actuator Matlab Optimization Code}

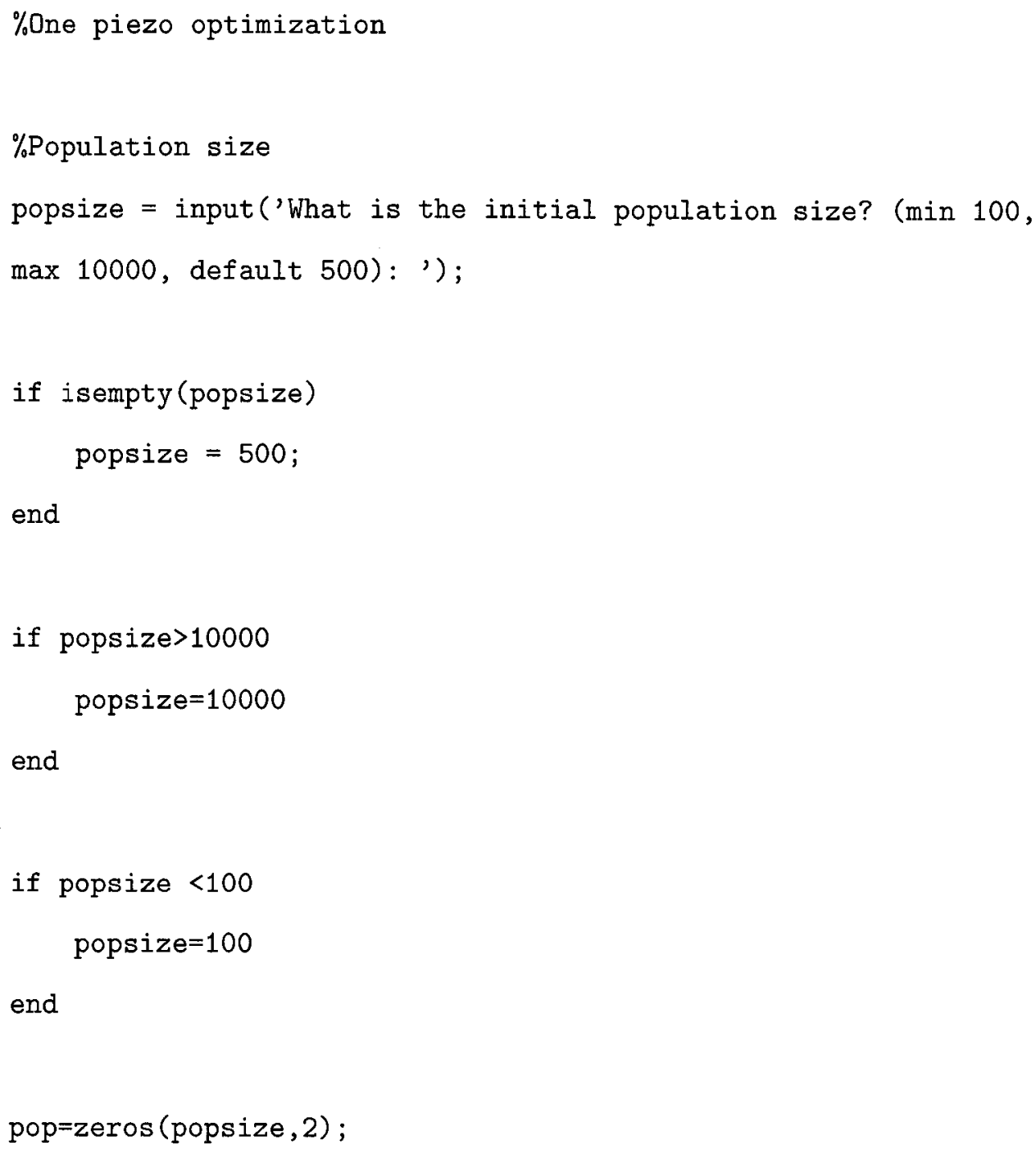




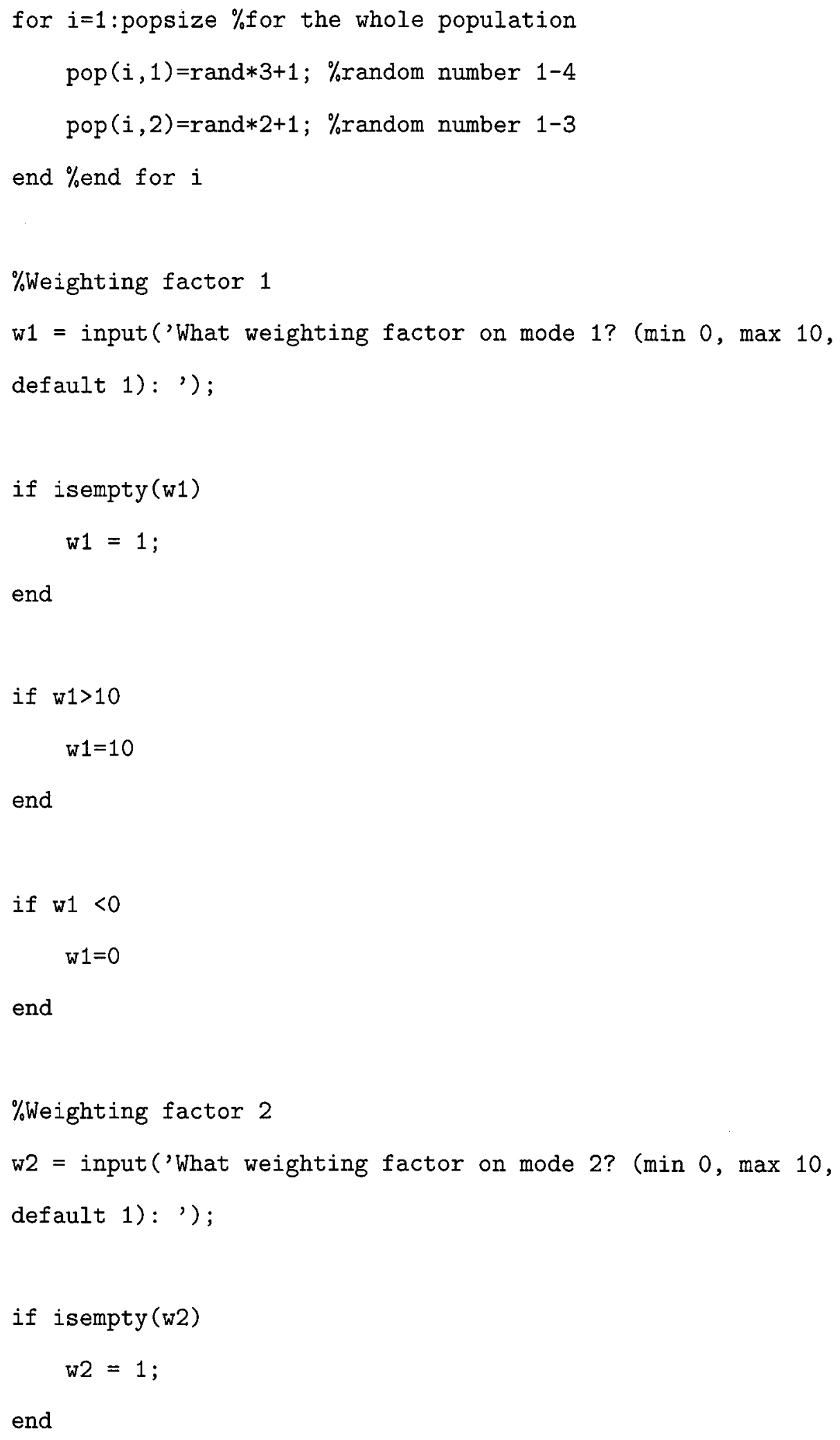




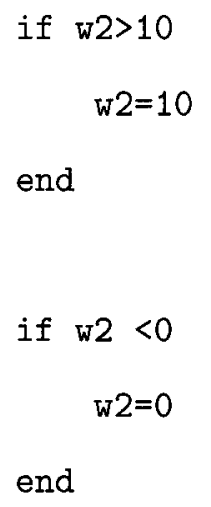




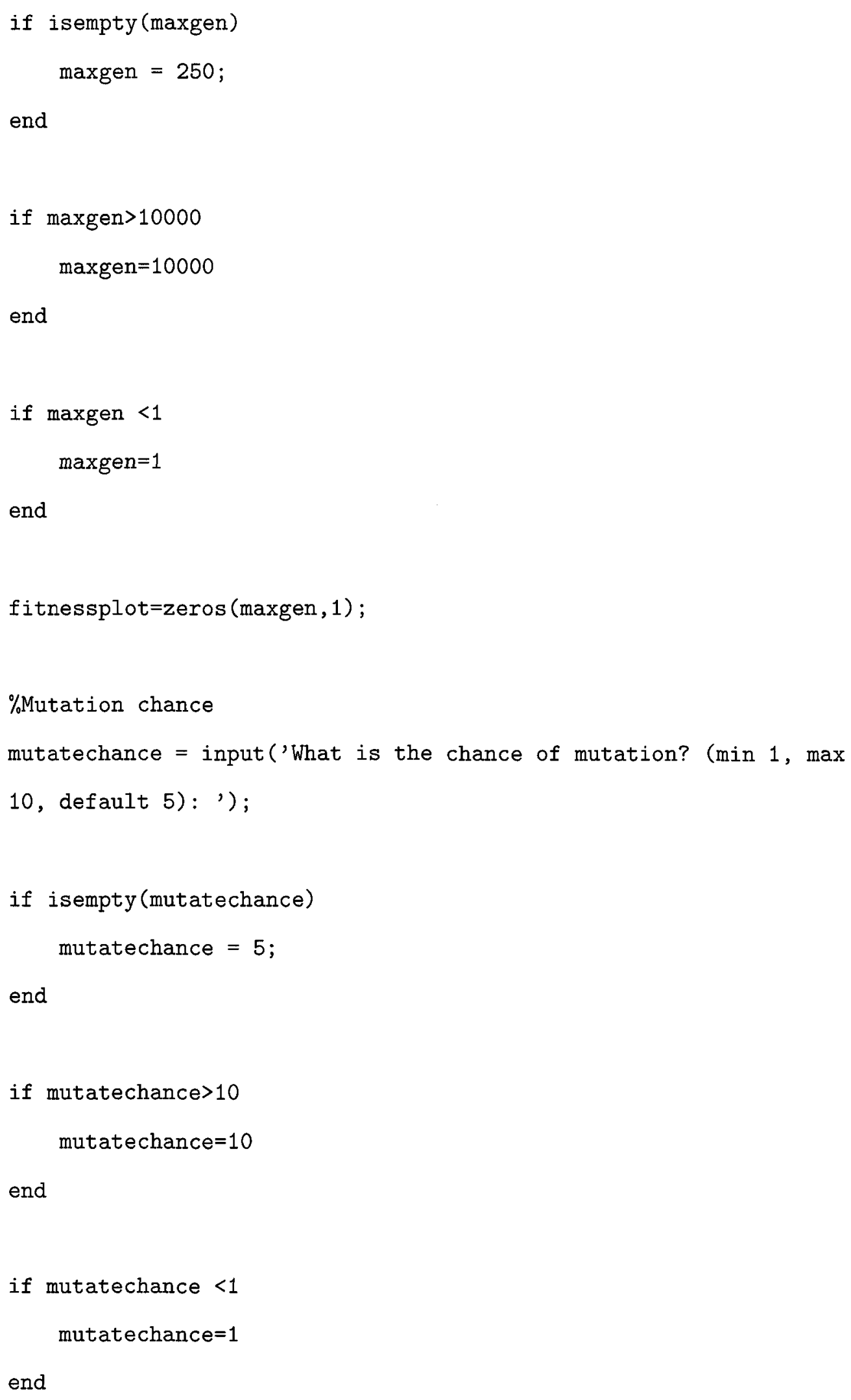


fitness=zeros $($ popsize, 1$)$;

\%Evaluate current values

a1=zeros $(1,55) ; \quad a 2=z \operatorname{eros}(1,55) ; \quad a 3=\operatorname{zeros}(1,55)$;

$\mathrm{a} 1(1)=74.72 ; \mathrm{a} 1(2)=9.59 ; \mathrm{a} 1(3)=-5.90 ; \mathrm{a} 1(4)=-0.82 ; \mathrm{a} 1(5)=-1.98 ;$

$\mathrm{a} 1(6)=-1.81 ; \mathrm{a} 1(7)=-0.20 ; \mathrm{a} 1(8)=0.07 ; \mathrm{a} 1(9)=1.20 ; \mathrm{a} 1(10)=-0.25 ;$

$\mathrm{a} 2(1)=68.87 ; \quad \mathrm{a} 2(2)=-22.10 ; \quad \mathrm{a} 2(3)=6.38 ; \quad \mathrm{a} 2(4)=-7.15 ; \quad \mathrm{a} 2(5)=-4.76$;

$\mathrm{a} 2(6)=0.49 ; \quad \mathrm{a} 2(7)=0.50 ; \mathrm{a} 2(8)=5.71 ; \mathrm{a} 2(9)=-8.88 ; \mathrm{a} 2(10)=1.45 ;$

$\mathrm{a} 2(11)=0.13 ; \mathrm{a} 2(12)=1.49 ; \mathrm{a} 2(13)=2.83 ; \mathrm{a} 2(14)=-2.34 ; \mathrm{a} 2(15)=0.65$;

$\mathrm{a} 2(16)=0.01 ; \mathrm{a} 2(17)=0.08 ; \mathrm{a} 2(18)=0.33 ; \mathrm{a} 2(19)=0.36 ; \mathrm{a} 2(20)=-0.31 ;$

$\mathrm{a} 2(21)=0.11 ; \mathrm{a} 2(22)=0.003 ; \mathrm{a} 2(23)=-0.0586 ; \mathrm{a} 2(24)=-0.0166$;

$\mathrm{a} 2(25)=0.0083 ; \mathrm{a} 2(26)=0.0317 ; \mathrm{a} 2(27)=-0.0508 ; \mathrm{a} 2(28)=0.0155$;

$\mathrm{a} 2(29)=-0.0015 ; \mathrm{a} 2(30)=-0.0157 ; \mathrm{a} 2(31)=-0.0094 ; \mathrm{a} 2(32)=-0.0015$;

a2 $(33)=-0.00006 ;$ a2 $(34)=0.0036 ; \mathrm{a} 2(35)=-0.0056 ; \mathrm{a} 2(36)=0.0027$;

a2 $(37)=0.0010 ; \quad$ a2 $(38)=-0.0030 ; \quad a 2(39)=0.0035 ; \quad a 2(40)=-0.0022$;

a2 $(41)=0.0001 ; a 2(42)=-0.000006 ; a 2(43)=-0.0023 ; a 2(44)=0.0047$;

a2 $(45)=0.000087$;

$\mathrm{a} 3(1)=43.61 ; \quad \mathrm{a} 3(2)=-67.51 ; \mathrm{a} 3(3)=148.49 ; \quad \mathrm{a} 3(4)=11.99 ; \mathrm{a} 3(5)=5.74 ;$

$\mathrm{a} 3(6)=33.03 ; \mathrm{a} 3(7)=-0.418 ; \mathrm{a} 3(8)=-6.844 ; \mathrm{a} 3(9)=-4.380$;

$\mathrm{a} 3(10)=-2.4375 ; \mathrm{a} 3(11)=0.1942 ; \mathrm{a} 3(12)=-0.3020 ; \mathrm{a} 3(13)=0.6289 ;$

$\mathrm{a} 3(14)=0.1898 ; \mathrm{a} 3(15)=-2.0013 ; \mathrm{a} 3(16)=0$; 
for $i=1:$ popsize

\%NOTE: 2xy, remove later

fitness $1=$ w $1 *(a 1(1)+(a 1(2) * p o p(i, 1))+(a 1(3) * p o p(i, 2))+(a 1(4) * p o p(i, 1)-2)+$ $2 *(a 1(5) * \operatorname{pop}(i, 1) * \operatorname{pop}(i, 2))+(a 1(6) * \operatorname{pop}(i, 2)-2)+(a 1(7) * p o p(i, 1)-3)+(a 1(8) *$ $\left.\operatorname{pop}(i, 1)^{\wedge} 2 * \operatorname{pop}(i, 2)\right)+\left(a 1(9) * \operatorname{pop}(i, 1) * \operatorname{pop}(i, 2)^{\wedge} 2\right)+(a 1(10) * p o p(i, 2) \wedge 3)+$ $\left(\right.$ a1 (11) *pop $\left.(i, 1)^{-} 4\right)+\left(\right.$ a. $\left.(12) * p o p(i, 1)^{\wedge} 3 * \operatorname{pop}(i, 2)\right)+\left(\right.$ a1 (13)*pop $(i, 1)^{\wedge} 2 *$ pop $\left.(i, 2)^{-} 2\right)+($ a1 (14)*pop $(i, 1) * \operatorname{pop}(i, 2) \sim 3)+($ a1 (15)*pop $(i, 2) \sim 4)+($ a1 (16)* $\left.\operatorname{pop}(i, 1)^{\sim} 5\right)+\left(\operatorname{a1}(17) * \operatorname{pop}(i, 1)^{-} 4 * \operatorname{pop}(i, 2)\right)+\left(\mathrm{a} 1(18) * \operatorname{pop}(i, 1)^{\sim} 3 * \operatorname{pop}(i, 2)^{\wedge} 2\right)+$

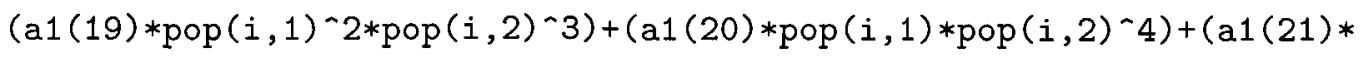
pop $(i, 2)-5)+(a 1(22) * p o p(i, 1)-6)+(a 1(23) * p o p(i, 1)-5 * p o p(i, 2))+(a 1(24) *$

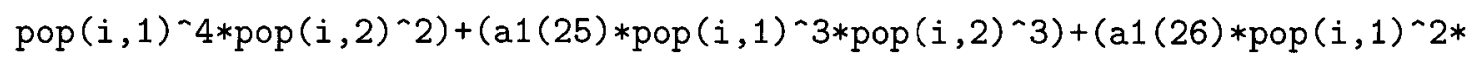
pop $(i, 2) \wedge 4)+\left(\right.$ a1 (27)*pop $\left.(i, 1) * p o p(i, 2)^{\wedge} 5\right)+\left(\right.$ a1 (28)*pop $\left.(i, 2)^{\wedge} 6\right)+($ a1 (29)* $\left.\operatorname{pop}(i, 1)^{\wedge} 7\right)+\left(\mathrm{a} 1(30) * \operatorname{pop}(i, 1)^{\sim} 6 * \operatorname{pop}(i, 2)\right)+\left(\mathrm{a} 1(31) * \mathrm{pop}(i, 1)^{\wedge} 5 * \operatorname{pop}(i, 2)^{\wedge} 2\right)+$

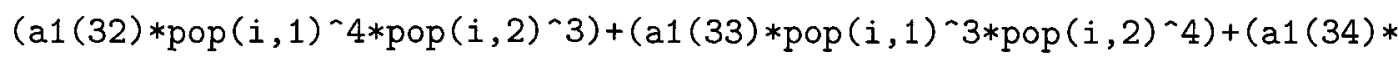
pop $\left.(i, 1)^{\sim} 2 * \operatorname{pop}(i, 2)^{-} 5\right)+\left(a 1(35) * p o p(i, 1) * p o p(i, 2)^{-} 6\right)+\left(a 1(36) * p o p(i, 2)^{\wedge} 7\right)+$ $($ a1 (37) *pop $(i, 1)-8)+($ a1 (38) *pop $(i, 1) \wedge 7 * \operatorname{pop}(i, 2))+($ a1 (39) *pop $(i, 1) \wedge 6 *$ pop $\left.(i, 2)^{\wedge} 2\right)+\left(a 1(40) * p o p(i, 1)^{\wedge} 5 * p o p(i, 2)^{\wedge} 3\right)+\left(a 1(41) * p o p(i, 1)^{\wedge} 4 * p o p(i, 2)^{\wedge} 4\right)+$

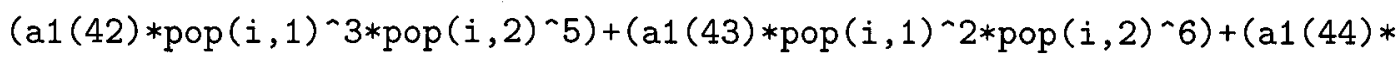
$\left.\operatorname{pop}(i, 1) * \operatorname{pop}(i, 2)^{\wedge} 7\right)+(a 1(45) * \operatorname{pop}(i, 2) \wedge 8)+(a 1(46) * p o p(i, 1) \wedge 9)+(a 1(47) *$ pop $\left.(i, 1)^{\sim} 8 * \operatorname{pop}(i, 2)\right)+\left(a 1(48) * \operatorname{pop}(i, 1)^{\sim} 7 * \operatorname{pop}(i, 2)^{\sim} 2\right)+\left(\right.$ a1 $(49) * \operatorname{pop}(i, 1)^{\wedge} 6 *$ pop $\left.(i, 2)^{\wedge} 3\right)+\left(\right.$ a1 $(50) * p o p(i, 1)^{\wedge} 5 *$ pop $\left.(i, 2)^{\wedge} 4\right)+\left(\right.$ a. $\left.(51) * p o p(i, 1)^{\wedge} 4 * p o p(i, 2)^{\wedge} 5\right)+$ (a1 (52) *pop (i , 1) ^3*pop $\left.(i, 2)^{-} 6\right)+\left(\right.$ a1 (53) *pop (i, 1) $2 *$ pop $\left.(i, 2)^{\sim} 7\right)+($ a1 (54)* $\left.\left.\operatorname{pop}(i, 1) * \operatorname{pop}(i, 2)^{-} 8\right)+\left(\operatorname{a1}(55) * \operatorname{pop}(i, 2)^{\wedge} 9\right)\right)$;

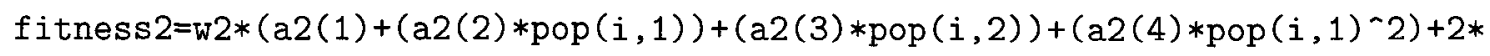
$(a 2(5) * p o p(i, 1) * p o p(i, 2))+(a 2(6) * p o p(i, 2)-2)+(a 2(7) * p o p(i, 1)-3)+(a 2(8) *$

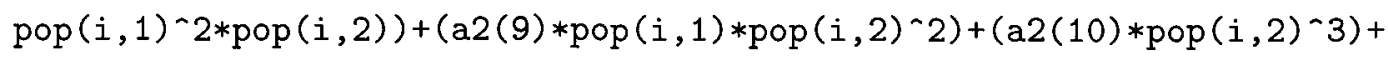

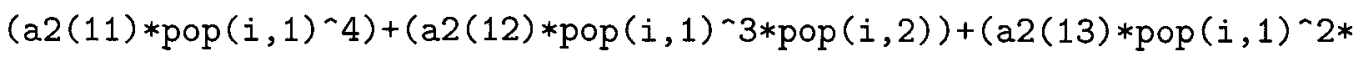
pop $\left.(i, 2)^{\wedge} 2\right)+\left(\right.$ a2 $\left.(14) * p o p(i, 1) * p o p(i, 2)^{\wedge} 3\right)+\left(a 2(15) * p o p(i, 2)^{\wedge} 4\right)+($ a2 (16)* 
$\left.\operatorname{pop}(i, 1)^{-} 5\right)+(a 2(17) * \operatorname{pop}(i, 1)-4 * p o p(i, 2))+(a 2(18) * p o p(i, 1)-3 * p o p(i, 2)-2)+$ $\left(\right.$ a2 (19) *pop $\left.(i, 1)^{\wedge} 2 * \operatorname{pop}(i, 2)^{\wedge} 3\right)+\left(\right.$ a2 (20)*pop $\left.(i, 1) * p o p(i, 2)^{\wedge} 4\right)+($ a2 (21)* pop $\left.(i, 2)^{\wedge} 5\right)+\left(a 2(22) * p o p(i, 1)^{-} 6\right)+(a 2(23) * p o p(i, 1) \wedge 5 * p o p(i, 2))+(a 2(24) *$

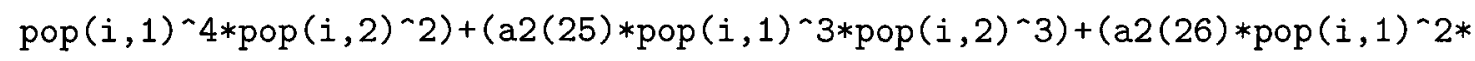
pop $\left.(i, 2)^{-4}\right)+\left(a 2(27) * p o p(i, 1) * p o p(i, 2)^{\wedge} 5\right)+(a 2(28) * p o p(i, 2)-6)+(a 2(29) *$ pop $\left.(i, 1)^{\wedge} 7\right)+\left(a 2(30) * p o p(i, 1)^{-} 6 * p o p(i, 2)\right)+\left(a 2(31) * p o p(i, 1)^{-} 5 * p o p(i, 2)^{-} 2\right)+$ $\left(\right.$ a2 (32) *pop $\left.(i, 1)^{-} 4 * \operatorname{pop}(i, 2)^{-}-3\right)+\left(\right.$ a2 (33) *pop $\left.(i, 1)^{-} 3 * \operatorname{pop}(i, 2)^{-} 4\right)+($ a2 (34)* pop $\left.(i, 1)^{\wedge} 2 * \operatorname{pop}(i, 2)^{\wedge} 5\right)+(a 2(35) * \operatorname{pop}(i, 1) * \operatorname{pop}(i, 2)-6)+\left(a 2(36) * \operatorname{pop}(i, 2)^{-} 7\right)+$ $\left(\right.$ a2 (37) *pop $\left.(i, 1)^{-} 8\right)+\left(\right.$ a2 (38)*pop $(i, 1)^{\wedge} 7 *$ pop $\left.(i, 2)\right)+\left(\right.$ a2 (39)*pop $(i, 1)^{-} 6 *$ pop $\left.(i, 2)^{\wedge} 2\right)+\left(\right.$ a2 $(40) * p o p(i, 1)^{\wedge} 5 *$ pop $\left.(i, 2)^{\wedge} 3\right)+\left(\right.$ a2 (41)*pop $(i, 1)^{\wedge} 4 *$ pop $\left.(i, 2)^{\wedge} 4\right)+\left(a 2(42) * p o p(i, 1)^{\wedge} 3 * p o p(i, 2) \wedge 5\right)+\left(a 2(43) * p o p(i, 1)^{\wedge} 2 *\right.$ pop $(i, 2) \wedge 6)+\left(a 2(44) * p o p(i, 1) * p o p(i, 2)^{\wedge} 7\right)+(a 2(45) * p o p(i, 2) \wedge 8)+(a 2(46) *$ pop $\left.(i, 1)^{\wedge} 9\right)+\left(a 2(47) * p o p(i, 1)^{-} 8 * p o p(i, 2)\right)+\left(a 2(48) * p o p(i, 1)^{\wedge} 7 * p o p(i, 2)^{\wedge} 2\right)+$

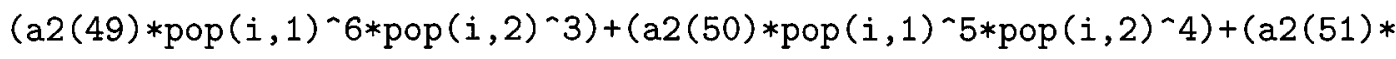

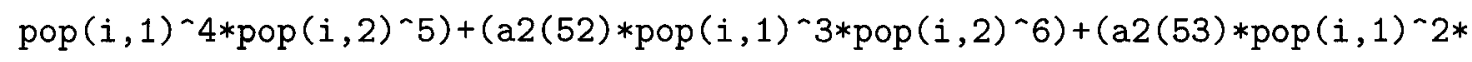
pop $\left.\left.(i, 2)^{\sim} 7\right)+\left(a 2(54) * p o p(i, 1) * p o p(i, 2)^{\wedge} 8\right)+\left(a 2(55) * p o p(i, 2)^{\wedge} 9\right)\right)$;

fitness $3=w 3 *(a 3(1)+(a 3(2) * p o p(i, 1))+(a 3(3) * p o p(i, 2))+(a 3(4) * p o p(i, 1)-2)+$ $2 *(a 3(5) * \operatorname{pop}(i, 1) * \operatorname{pop}(i, 2))+\left(a 3(6) * \operatorname{pop}(i, 2)^{\sim} 2\right)+\left(a 3(7) * \operatorname{pop}(i, 1)^{-} 3\right)+$ $\left(\right.$ a3 (8)*pop $\left.(i, 1)^{\wedge} 2 * \operatorname{pop}(i, 2)\right)+\left(\right.$ a3 $\left.(9) * \operatorname{pop}(i, 1) * \operatorname{pop}(i, 2)^{\wedge} 2\right)+($ a3 (10)* pop $(i, 2) \sim 3)+\left(\right.$ a3 (11) *pop $\left.(i, 1)^{\wedge} 4\right)+($ a3 $(12) * p o p(i, 1)-3 * p o p(i, 2))+(a 3(13) *$ pop $\left.(i, 1)^{\wedge} 2 * \operatorname{pop}(i, 2)^{\wedge} 2\right)+\left(a 3(14) * p o p(i, 1) * p o p(i, 2)^{\wedge} 3\right)+\left(\right.$ a3 (15)*pop $\left.(i, 2)^{\wedge} 4\right)+$ $\left(\right.$ a3 (16)*pop $\left.(i, 1)^{\wedge} 5\right)+\left(\right.$ a3 (17) *pop $(i, 1)^{\wedge} 4 *$ pop $\left.(i, 2)\right)+\left(\right.$ a3 (18)*pop $(i, 1)^{\wedge} 3 *$ pop $\left.(i, 2)^{\wedge} 2\right)+\left(a 3(19) * p o p(i, 1)^{\wedge} 2 * p o p(i, 2)^{\wedge} 3\right)+\left(a 3(20) * p o p(i, 1) * p o p(i, 2)^{\wedge} 4\right)+$

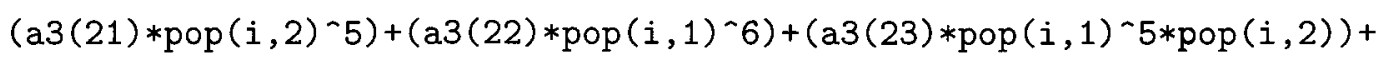

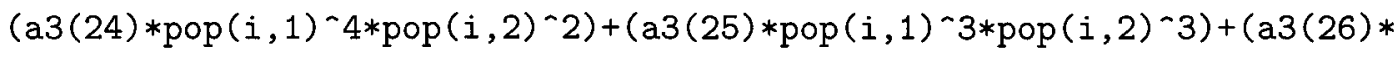
pop $\left.(i, 1)^{-} 2 * p o p(i, 2) \wedge 4\right)+(a 3(27) * p o p(i, 1) * p o p(i, 2) \wedge 5)+(a 3(28) * p o p(i, 2)-6)+$ $($ a3 (29)*pop $(i, 1) \sim 7)+($ a3 (30)*pop $(i, 1) \sim 6 *$ pop $(i, 2))+\left(\right.$ a3 (31)*pop $(i, 1)^{\wedge} 5 *$ pop $\left.(i, 2)^{\wedge} 2\right)+\left(a 3(32) * p o p(i, 1)^{\wedge} 4 * p o p(i, 2)^{\wedge} 3\right)+\left(a 3(33) * p o p(i, 1)^{\wedge} 3 * p o p(i, 2)^{\wedge} 4\right)+$ 


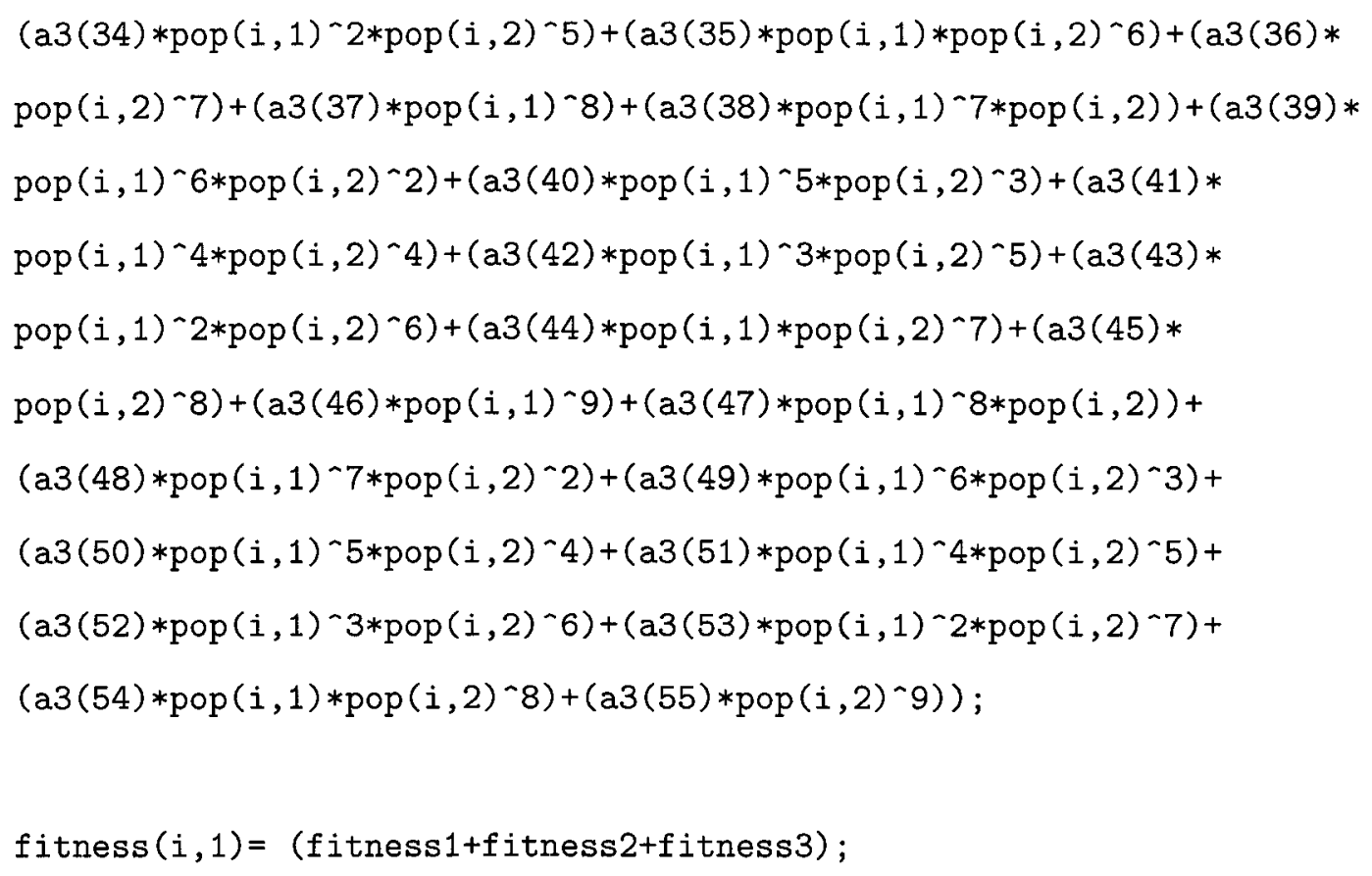

\%Make new population by breeding all posible combinations. Note that the $\%$ ten parents are part of the population (self-cross), although they are 
$\%$ subject to mutation (if favourable)

newpop=zeros $\left(\right.$ select $\left.^{`} 2,2\right)$; \%declare newpopulation matrix. Note for breeding all selected values, the number of possible

$\%$ combinations is select ${ }^{\sim} 2$.

generation $=0$;

while generation<maxgen

generation=generation+1; \% Increase number of generations by 1 .

for $r=1:$ select \%for each of the selected individuals primarycross=selection $(r)$; \%set each one in turn as the primary cross individual

for $i=1:$ select $\%$ for each other selected individual (including itself for self cross). This is the secondary cross.

level=(r-1)*select+i; \% find position in newpopulation matrix based on the primary and secondary cross.

alphax=rand; \%result of cross for $x$; 0 to 1 for alpha, weighting on primary vs. secondary cross.

alphay=rand; \%result of cross for y; 0 to 1 for alpha, weighting on primary vs. secondary cross.

newpop (leve1,1)=alphax*pop (primarycross , 1)+(1-alphax)*pop (selection (i) , 1) ;

$\%$ set allele to weighted value of primary cross and secondary cross newpop (level , 2) =alphay*pop (primarycross , 2) +(1-alphay) *pop (selection $(i), 2)$;

$\%$ set allele to weighted value of primary cross and secondary cross end $\%$ end for $i$ 
end \%end for $r$

pop=zeros $($ select` 2,2$) ; \%$ re-declare population matrix (need to resize from popsize to select ${ }^{-2}$ if first run)

fitness=zeros (select`2,1); \%re-declare fitness matrix (need to re-size)

pop=newpop; \%set population to crossed population

mutated=zeros $(1,2)$;

$\%$ Introduce a chance of mutation in the population before selection

$\%$ (since most mutations will be detrimental and therefore selected out)

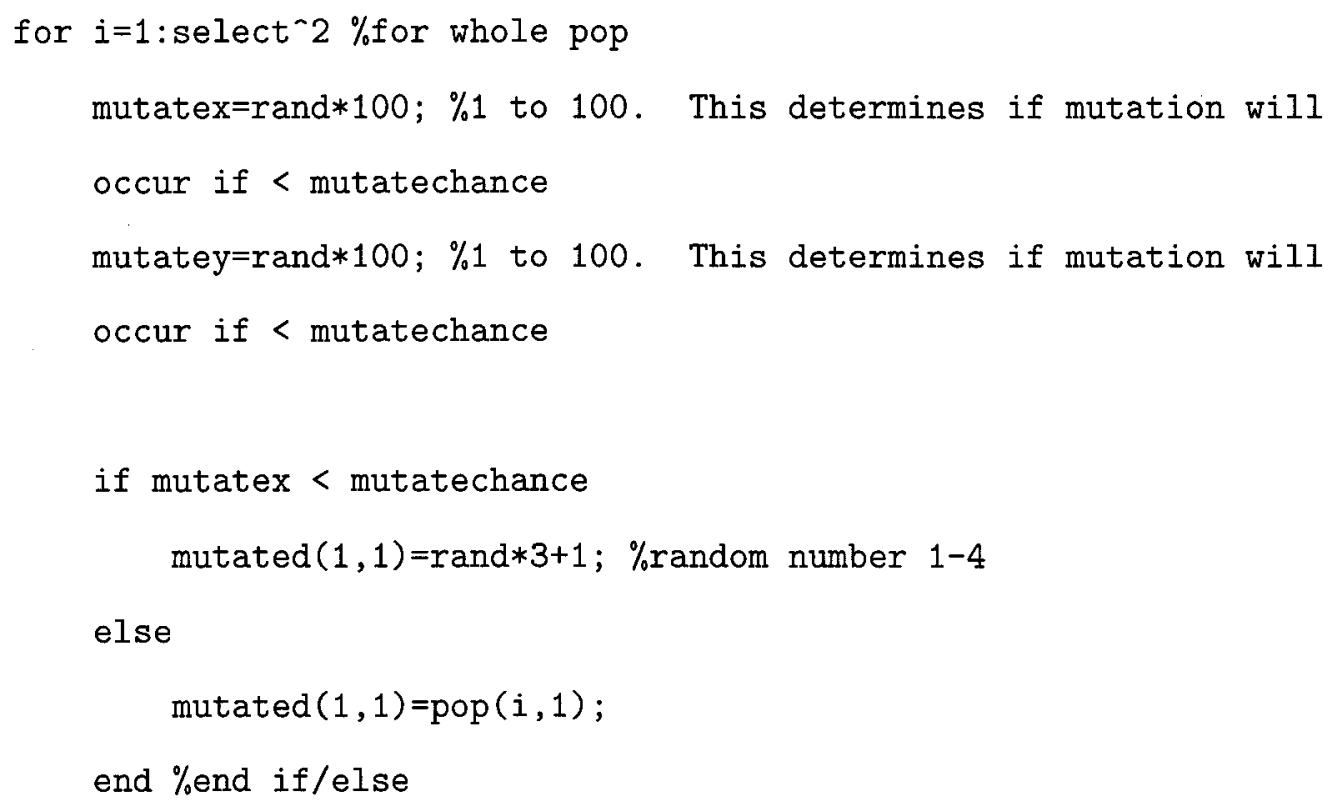




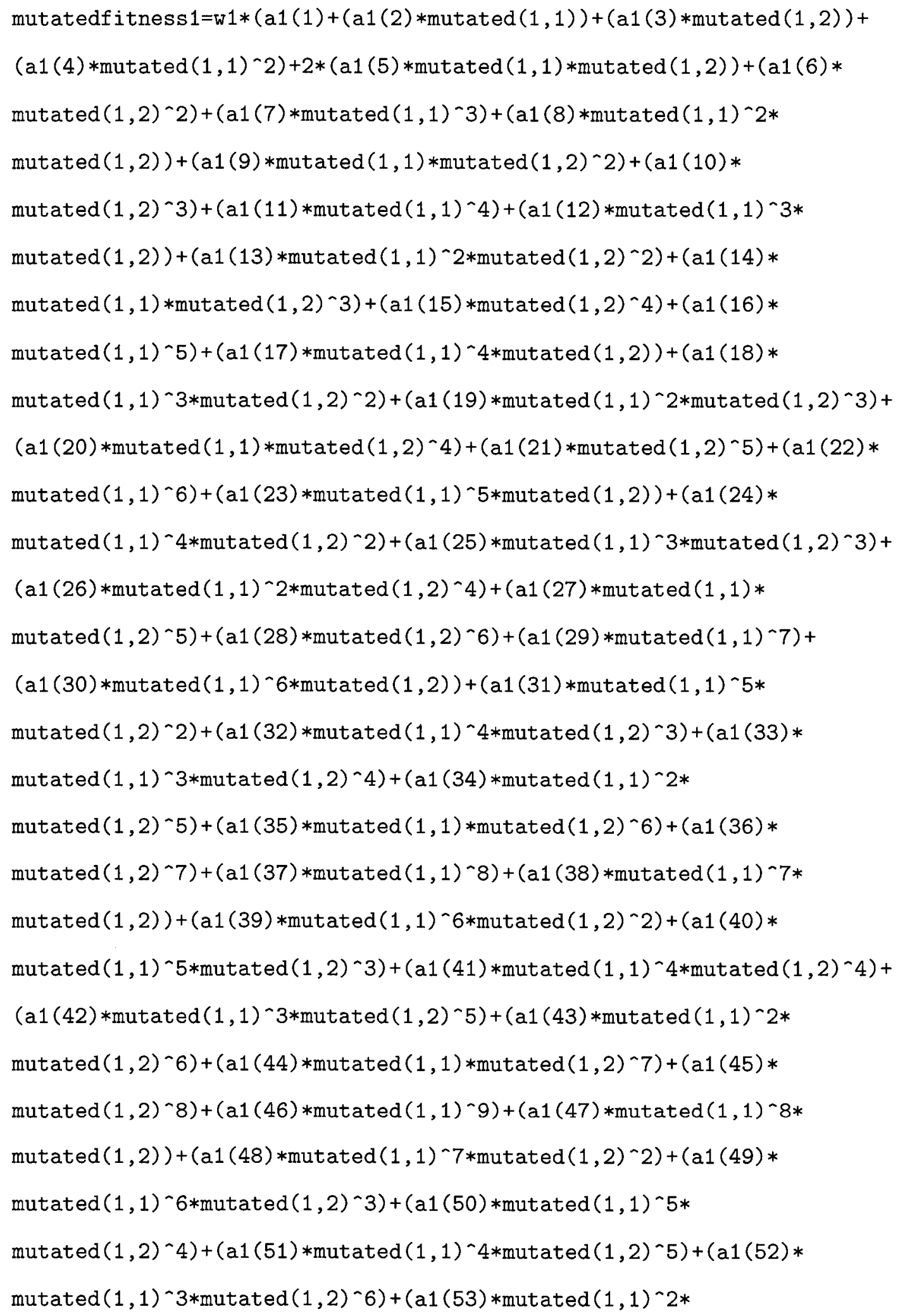


mutated $\left.(1,2)^{-} 7\right)+($ a1 (54) $*$ mutated $(1,1) * \operatorname{mutated}(1,2)-8)+\left(\right.$ a1 $\left.\left.(55) * \operatorname{mutated}(1,2)^{-}-9\right)\right)$;

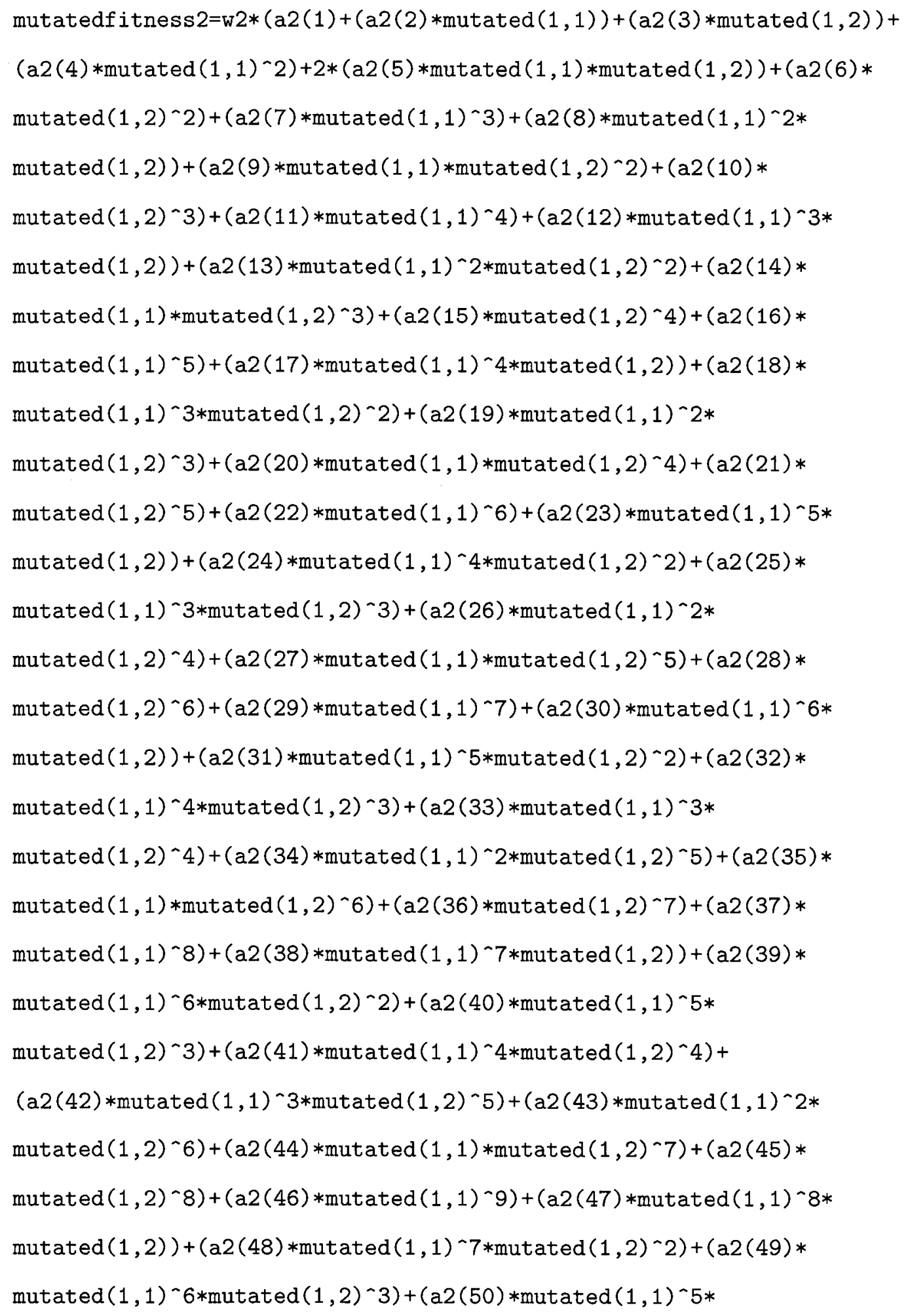




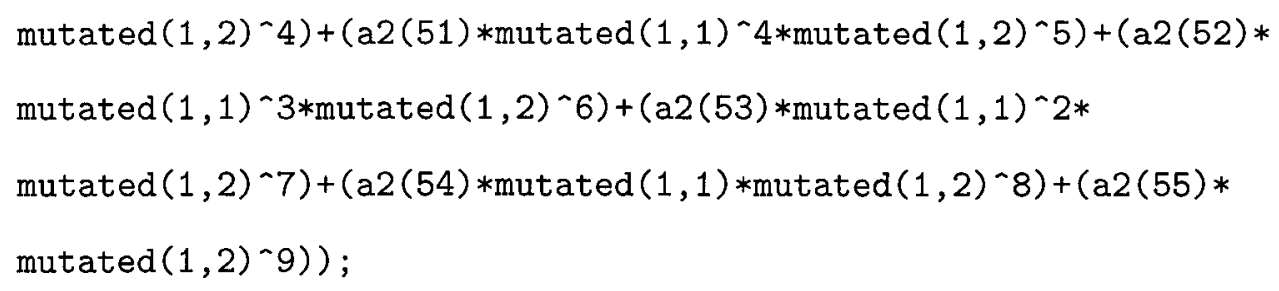




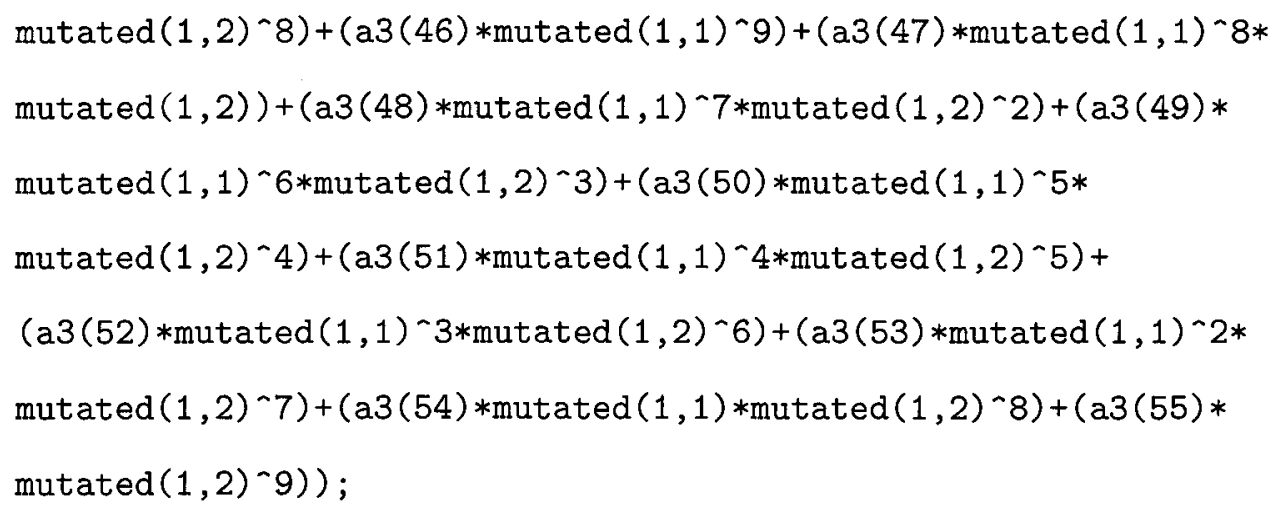


(a1 (45)*pop $\left.(i, 2)^{-} 8\right)+\left(\right.$ a1 (46)*pop $\left.(i, 1)^{\wedge} 9\right)+($ a1 (47) *pop (i, 1)^8* $\operatorname{pop}(i, 2))+\left(\right.$ a $\left.1(48) * \operatorname{pop}(i, 1)^{\wedge} 7 * \operatorname{pop}(i, 2) \sim 2\right)+\left(\right.$ a $1(49) * \operatorname{pop}(i, 1)^{\wedge} 6 *$ pop $\left.(i, 2)^{-} 3\right)+\left(\right.$ a $\left.1(50) * p o p(i, 1)^{\sim} 5 * \operatorname{pop}(i, 2)^{\wedge} 4\right)+\left(\mathrm{a} 1(51) * \operatorname{pop}(i, 1)^{\sim} 4 *\right.$ $\left.\operatorname{pop}(i, 2)^{\wedge} 5\right)+\left(\right.$ a1 (52)*pop $\left.(i, 1)^{\wedge} 3 * \operatorname{pop}(i, 2)^{\wedge} 6\right)+\left(\right.$ a1 (53) *pop $(i, 1)^{\wedge} 2 *$ $\left.\operatorname{pop}(i, 2)^{-} 7\right)+($ a1 (54) *pop $\left.(i, 1) * p o p(i, 2)-8)+\left(a 1(55) * \operatorname{pop}(i, 2)^{-} 9\right)\right)$;

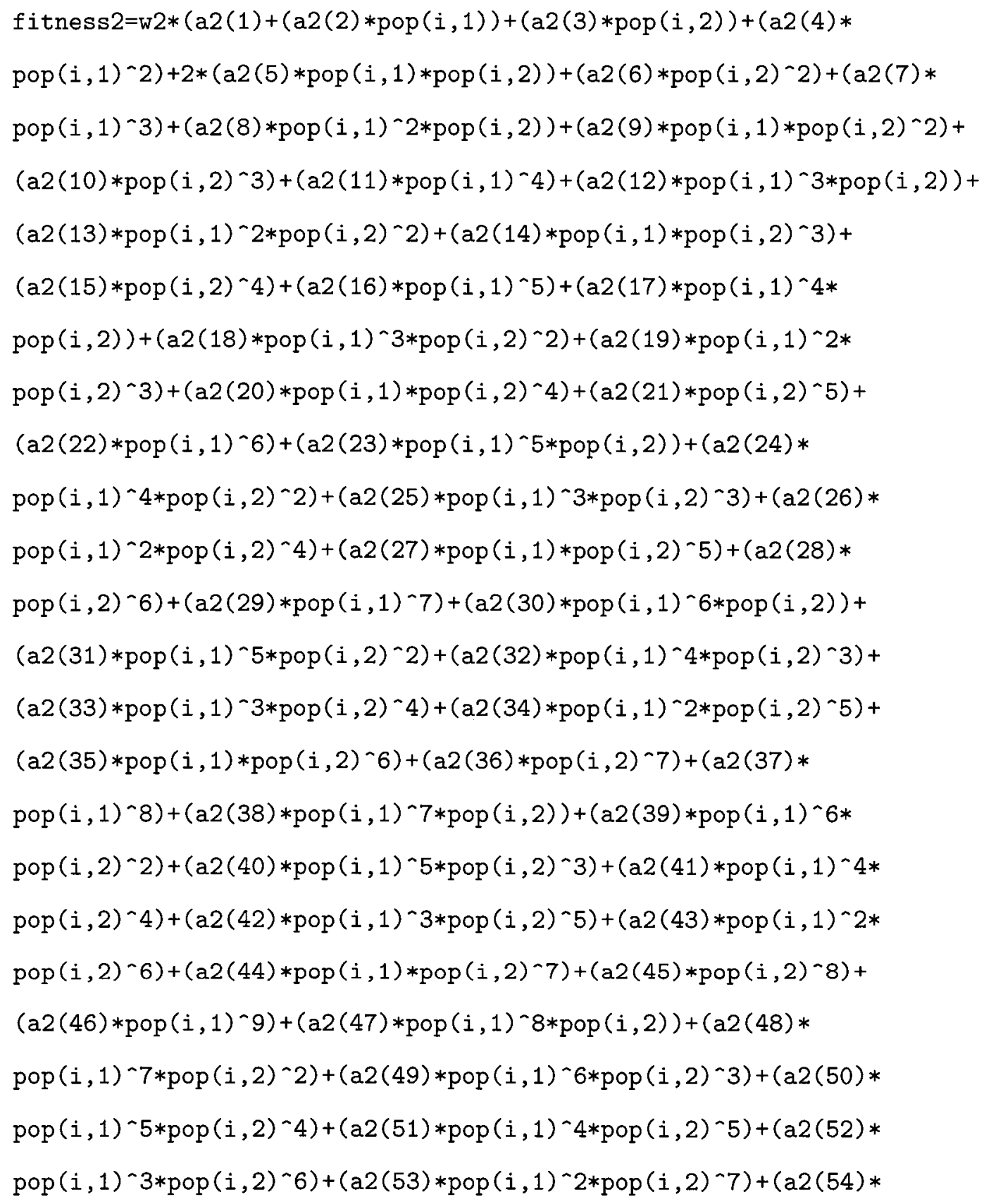




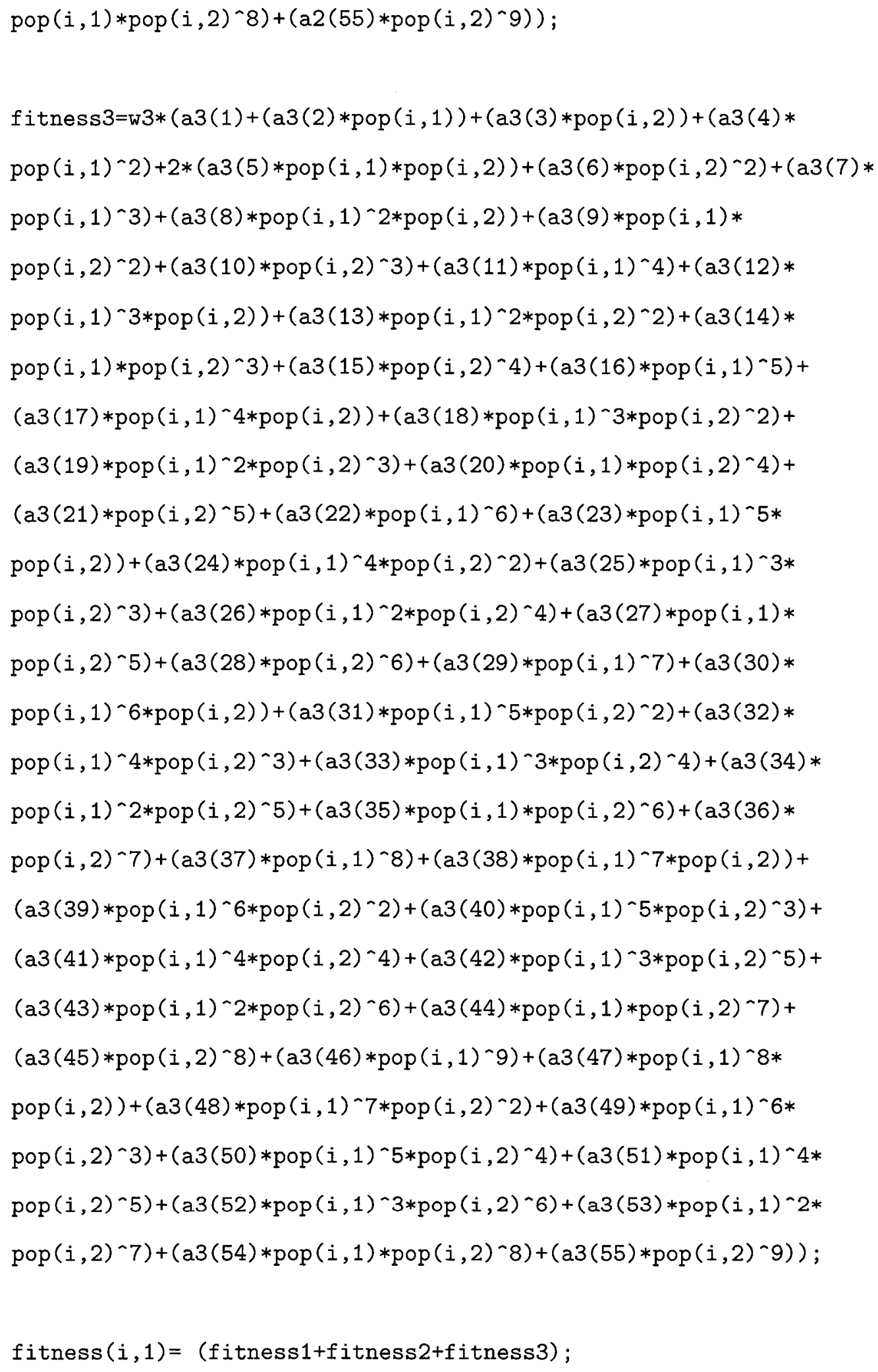


if mutatedfitness $>$ fitness $(i, 1)$

$$
\begin{aligned}
& \operatorname{pop}(i, 1)=\text { mutated }(1,1) ; \\
& \operatorname{pop}(i, 2)=\text { mutated }(1,2) ;
\end{aligned}
$$

end

end \%end for and mutation

\section{$\%$ determine fitness and re-select}

for $i=1:$ select ${ }^{\wedge} 2$

\%NOTE: $2 x y$, remove later

fitness $1=w 1 *(a 1(1)+(a 1(2) * p o p(i, 1))+(a 1(3) * p o p(i, 2))+(a 1(4) *$ $\left.\operatorname{pop}(i, 1)^{\wedge} 2\right)+2 *(a 1(5) * \operatorname{pop}(i, 1) * \operatorname{pop}(i, 2))+\left(\mathrm{a} 1(6) * \operatorname{pop}(i, 2)^{\wedge} 2\right)+(\mathrm{a} 1(7) *$ $\operatorname{pop}(i, 1) \wedge 3)+(a 1(8) * p o p(i, 1) \wedge 2 * p o p(i, 2))+(a 1(9) * p o p(i, 1) * p o p(i, 2) \wedge 2)+$ $($ a1 (10) *pop $(i, 2)-3)+($ a1 (11)*pop $(i, 1)-4)+($ a1 (12)*pop (i, 1) -3* pop $(i, 2))+\left(a 1(13) * p o p(i, 1)^{\wedge} 2 * \operatorname{pop}(i, 2)^{\wedge} 2\right)+(a 1(14) * p o p(i, 1) *$ $\operatorname{pop}(i, 2)-3)+($ a1 (15)*pop $(i, 2)-4)+(a 1(16) * \operatorname{pop}(i, 1)-5)+($ a1 (17)* $\left.\operatorname{pop}(i, 1)^{\wedge} 4 * \operatorname{pop}(i, 2)\right)+\left(a 1(18) * \operatorname{pop}(i, 1)^{\wedge} 3 * \operatorname{pop}(i, 2)^{\wedge} 2\right)+(\mathrm{a} 1(19) *$ $\operatorname{pop}(i, 1) \sim 2 * \operatorname{pop}(i, 2)-3)+($ a1 (20)*pop (i, 1) *pop $(i, 2) \sim 4)+($ a1 (21)* pop $\left.(i, 2)^{\wedge} 5\right)+\left(a 1(22) * p o p(i, 1)^{\wedge} 6\right)+\left(a 1(23) * p o p(i, 1)^{\wedge} 5 * p o p(i, 2)\right)+$

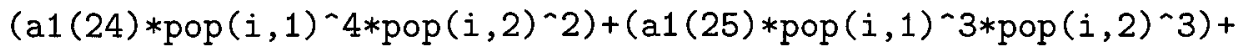
$\left(\right.$ a1 (26) *pop $\left.(i, 1)^{\wedge} 2 * \operatorname{pop}(i, 2)^{\wedge} 4\right)+($ a1 (27)*pop (i, 1)*pop (i, 2)^5)+ $\left(\right.$ a1 (28) *pop $\left.(i, 2)^{-}-6\right)+\left(\right.$ a1 (29)*pop $\left.(i, 1)^{-} 7\right)+($ a1 (30)*pop $(i, 1)-6 *$ pop $(i, 2))+\left(\right.$ a1 (31) *pop $(i, 1)^{\wedge} 5 *$ pop $\left.(i, 2) \wedge 2\right)+($ a1 (32)*pop $(i, 1) \wedge 4 *$ $\left.\operatorname{pop}(i, 2)^{\wedge} 3\right)+\left(\right.$ a1 (33)*pop $\left.(i, 1) ` 3 * \operatorname{pop}(i, 2)^{\wedge} 4\right)+\left(\mathrm{a} 1(34) * \operatorname{pop}(i, 1)^{\wedge} 2 *\right.$ $\left.\operatorname{pop}(i, 2)^{\wedge} 5\right)+\left(\mathrm{a} 1(35) * \operatorname{pop}(i, 1) * \operatorname{pop}(i, 2)^{\wedge} 6\right)+\left(\mathrm{a} 1(36) * \mathrm{pop}(i, 2)^{\wedge} 7\right)+$ $\left(\right.$ a $\left.1(37) * \operatorname{pop}(i, 1)^{\wedge} 8\right)+\left(\right.$ a1 $\left.(38) * \operatorname{pop}(i, 1)^{\sim} 7 * \operatorname{pop}(i, 2)\right)+($ a1 (39)*

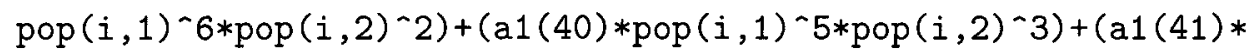
pop $\left.(i, 1)^{\wedge} 4 * \operatorname{pop}(i, 2)^{\wedge} 4\right)+\left(\right.$ a1 (42)*pop $\left.(i, 1)^{\wedge} 3 * \operatorname{pop}(i, 2)^{\wedge} 5\right)+($ a1 (43)* 


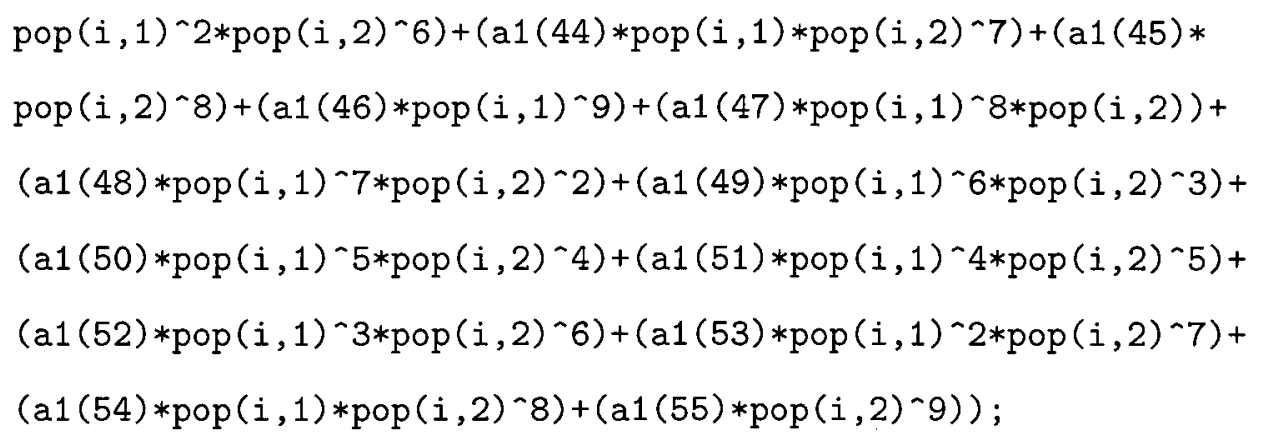


$\left.\operatorname{pop}(i, 2)^{\wedge} 5\right)+\left(\mathrm{a} 2(52) * \operatorname{pop}(i, 1)^{\wedge} 3 * \operatorname{pop}(i, 2)^{\wedge} 6\right)+\left(\mathrm{a} 2(53) * \operatorname{pop}(i, 1)^{\wedge} 2 *\right.$
$\left.\left.\operatorname{pop}(i, 2)^{\wedge} 7\right)+(\mathrm{a} 2(54) * \operatorname{pop}(i, 1) * \operatorname{pop}(i, 2) \wedge 8)+\left(\mathrm{a} 2(55) * \operatorname{pop}(i, 2)^{\wedge} 9\right)\right)$

fitness $3=$ w $3 *(a 3(1)+(a 3(2) *$ pop $(i, 1))+(a 3(3) *$ pop $(i, 2))+(a 3(4) *$ pop $\left.(i, 1)^{-} 2\right)+2 *(a 3(5) * \operatorname{pop}(i, 1) * p o p(i, 2))+\left(a 3(6) * p o p(i, 2)^{\wedge} 2\right)+$ (a3 (7) *pop $\left.(i, 1)^{\wedge} 3\right)+($ a3 (8) *pop $(i, 1) \wedge 2 * p o p(i, 2))+($ a3 (9) *pop $(i, 1) *$ $\left.\operatorname{pop}(i, 2)^{\wedge} 2\right)+(a 3(10) * \operatorname{pop}(i, 2) \wedge 3)+\left(a 3(11) * p o p(i, 1)^{\wedge} 4\right)+(a 3(12) *$ $\left.\operatorname{pop}(i, 1)^{-3 * \operatorname{pop}}(i, 2)\right)+\left(\right.$ a3 (13) *pop $\left.(i, 1)^{\sim} 2 * \operatorname{pop}(i, 2)^{\sim} 2\right)+($ a3 (14)* $\left.\operatorname{pop}(i, 1) * \operatorname{pop}(i, 2)^{-3}\right)+\left(\mathrm{a} 3(15) * \operatorname{pop}(i, 2)^{\wedge} 4\right)+\left(\mathrm{a} 3(16) * \operatorname{pop}(i, 1)^{\wedge} 5\right)+$ $\left(\mathrm{a} 3(17) * \mathrm{pop}(i, 1)^{\wedge} 4 * \mathrm{pop}(i, 2)\right)+\left(\mathrm{a} 3(18) * \mathrm{pop}(i, 1)^{\wedge} 3 * \mathrm{pop}(i, 2)^{\wedge} 2\right)+$ (a3 (19)*pop (i, 1)^2*pop (i, 2)^3)+(a3(20)*pop (i, 1)*pop (i, 2)^4)+ (a3 (21)*pop $\left.(i, 2)^{\sim} 5\right)+\left(\right.$ a3 (22) *pop $\left.(i, 1)^{\wedge} 6\right)+($ a3 (23)*pop $(i, 1) \wedge 5 *$ $\operatorname{pop}(i, 2))+\left(\right.$ a3 $\left.(24) * \operatorname{pop}(i, 1)^{\wedge} 4 * \operatorname{pop}(i, 2)^{\wedge} 2\right)+\left(\right.$ a3 (25)*pop $(i, 1)^{\wedge} 3 *$ pop $\left.(i, 2)^{-} 3\right)+\left(\right.$ a3 $\left.(26) * \operatorname{pop}(i, 1)^{\wedge} 2 * \operatorname{pop}(i, 2)^{\wedge} 4\right)+($ a3 $(27) * \operatorname{pop}(i, 1) *$ pop $\left.(i, 2)^{\wedge} 5\right)+\left(\right.$ a3 (28)*pop $\left.(i, 2)^{\wedge} 6\right)+\left(\right.$ a3 (29)*pop $\left.(i, 1)^{\wedge} 7\right)+($ a3 (30)* pop $\left.(i, 1)^{-} 6 * \operatorname{pop}(i, 2)\right)+\left(\right.$ a3 (31) *pop (i, 1)`5*pop $\left.(i, 2)^{\wedge} 2\right)+($ a3 (32)*

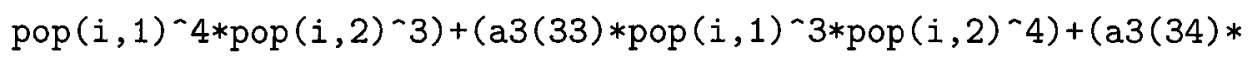

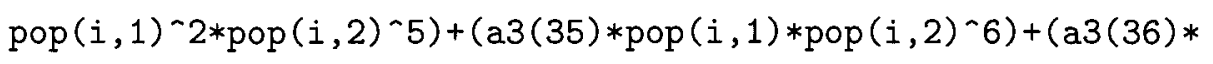
$\left.\operatorname{pop}(i, 2)^{\wedge} 7\right)+\left(\mathrm{a} 3(37) * \mathrm{pop}(i, 1)^{\sim} 8\right)+\left(\mathrm{a} 3(38) * \mathrm{pop}(\mathrm{i}, 1)^{\sim} 7 * \mathrm{pop}(\mathrm{i}, 2)\right)+$ (a3 (39)*pop (i, 1)^6*pop (i, 2)^2)+(a3(40)*pop (i, 1)^5*pop (i, 2)^3)+ $\left(\right.$ a3 (41) *pop $\left.(i, 1)^{\wedge} 4 * \operatorname{pop}(i, 2) \wedge 4\right)+\left(\right.$ a3 $\left.(42) * \operatorname{pop}(i, 1) \wedge 3 * \operatorname{pop}(i, 2)^{\wedge} 5\right)+$ $\left(\right.$ a3 (43) *pop $\left.(i, 1)^{`} 2 * \operatorname{pop}(i, 2)^{\wedge} 6\right)+\left(\right.$ a3 $\left.(44) * \operatorname{pop}(i, 1) * \operatorname{pop}(i, 2)^{\wedge} 7\right)+$ $\left(\right.$ a3 (45) *pop $\left.(i, 2)^{\wedge} 8\right)+\left(\right.$ a3 (46)*pop $\left.(i, 1)^{\wedge} 9\right)+\left(\right.$ a3 (47)*pop $(i, 1)^{\wedge} 8 *$ $\operatorname{pop}(i, 2))+\left(\right.$ a.3 (48)*pop $\left.(i, 1)^{\wedge} 7 * \operatorname{pop}(i, 2) ` 2\right)+\left(\right.$ a3 (49)*pop $(i, 1)^{\wedge} 6 *$ pop $(i, 2) \wedge 3)+(a 3(50) * p o p(i, 1) \wedge 5 * p o p(i, 2) \wedge 4)+(a 3(51) * p o p(i, 1) \wedge 4 *$ pop $\left.(i, 2)^{\wedge} 5\right)+\left(a 3(52) * \operatorname{pop}(i, 1)^{\wedge} 3 * \operatorname{pop}(i, 2)^{\wedge} 6\right)+\left(a 3(53) * p o p(i, 1)^{\wedge} 2 *\right.$ $\left.\left.\operatorname{pop}(i, 2)^{\sim} 7\right)+\left(a 3(54) * p o p(i, 1) * p o p(i, 2)^{\wedge} 8\right)+\left(a 3(55) * p o p(i, 2)^{-9}\right)\right)$;

fitness $(i, 1)=($ fitness $1+f i t n e s s 2+f i t n e s s ~ 3)$; 
end

bestvalue=max (fitness) \%display the current best individual fitness fitnessplot (generation) $=\max ($ fitness);

for $i=1:$ select $\%$ for the number of fitest individuals we wish to select $[C, I]=\max$ (fitness); \% find the index of the fitest individual selection( $i)=I$; \%store that index in the matrix selection fitness(I) $=0$; \%set fitness of that individual to zero so the loop will select the next fitest, etc.

end $\%$ end for $i$

end \%end of algorith while loop

$\mathrm{xcoordinate}=\mathrm{pop}(\operatorname{selection}(1), 1)$ ycoordinate=pop (selection $(1), 2)$

$\%$ Plot individual surface plots and combination with weighting factors

xplot=linspace $(1,4,100) ; y p l o t=l i n s p a c e(1,3,100)$;

$\mathbf{z} 1=\operatorname{zeros}(100,100) ; \quad z 2=\operatorname{zeros}(100,100) ; \quad z 3=\operatorname{zeros}(100,100)$;

$\mathbf{z} 4=\mathbf{z e r o s}(100,100)$;

for $i=1: 100 ; \%$ for $x$

for $j=1: 100$; $\%$ for $y$

$z 1(i, j)=(a 1(1)+(a 1(2) * x p l o t(i))+(a 1(3) * y p l o t(j))+(a 1(4) *$

$\operatorname{xplot}(i) \wedge 2)+2 *(a 1(5) * x p l o t(i) * y p l o t(j))+(a 1(6) * y p l o t(j) \wedge 2)+$

(a1 (7) *xplot (i)^3)+(a1(8)*xplot (i)^2*yplot (j))+(a1 (9)*

$\operatorname{xplot}(i) * y p l o t(j) \wedge 2)+(a 1(10) * y p l o t(j) \wedge 3)+(a 1(11) * \operatorname{xplot}(i) \wedge 4)+$ 


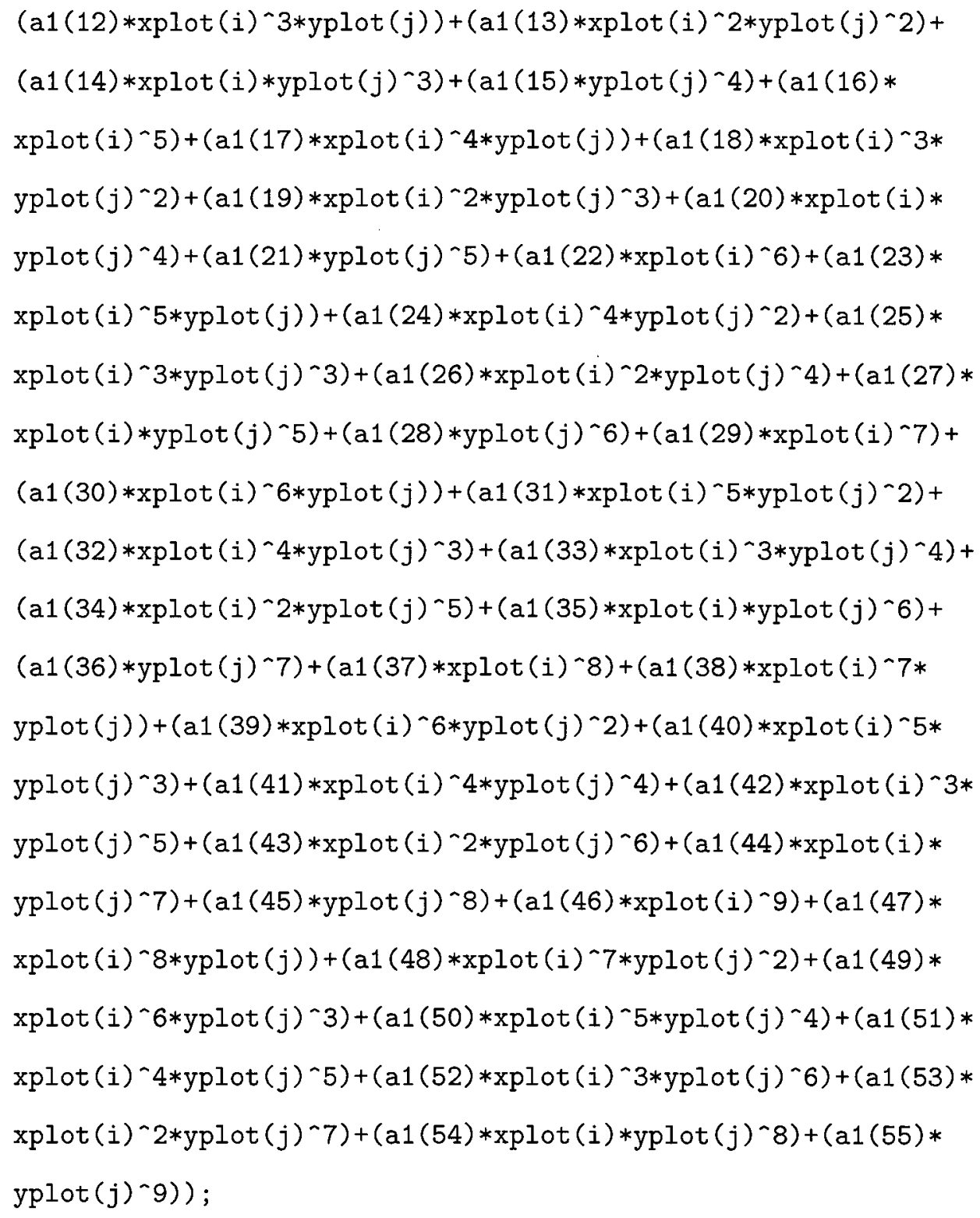




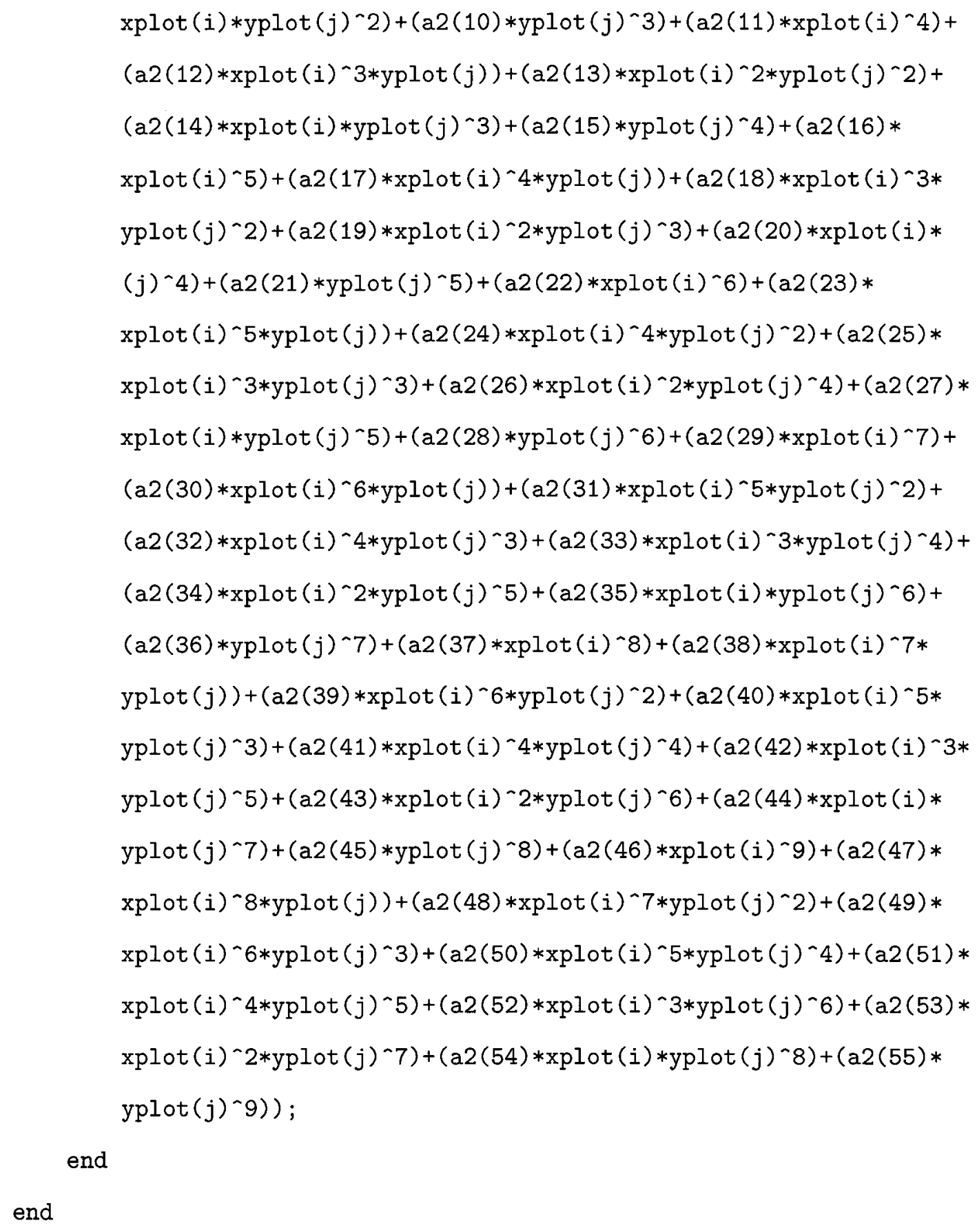




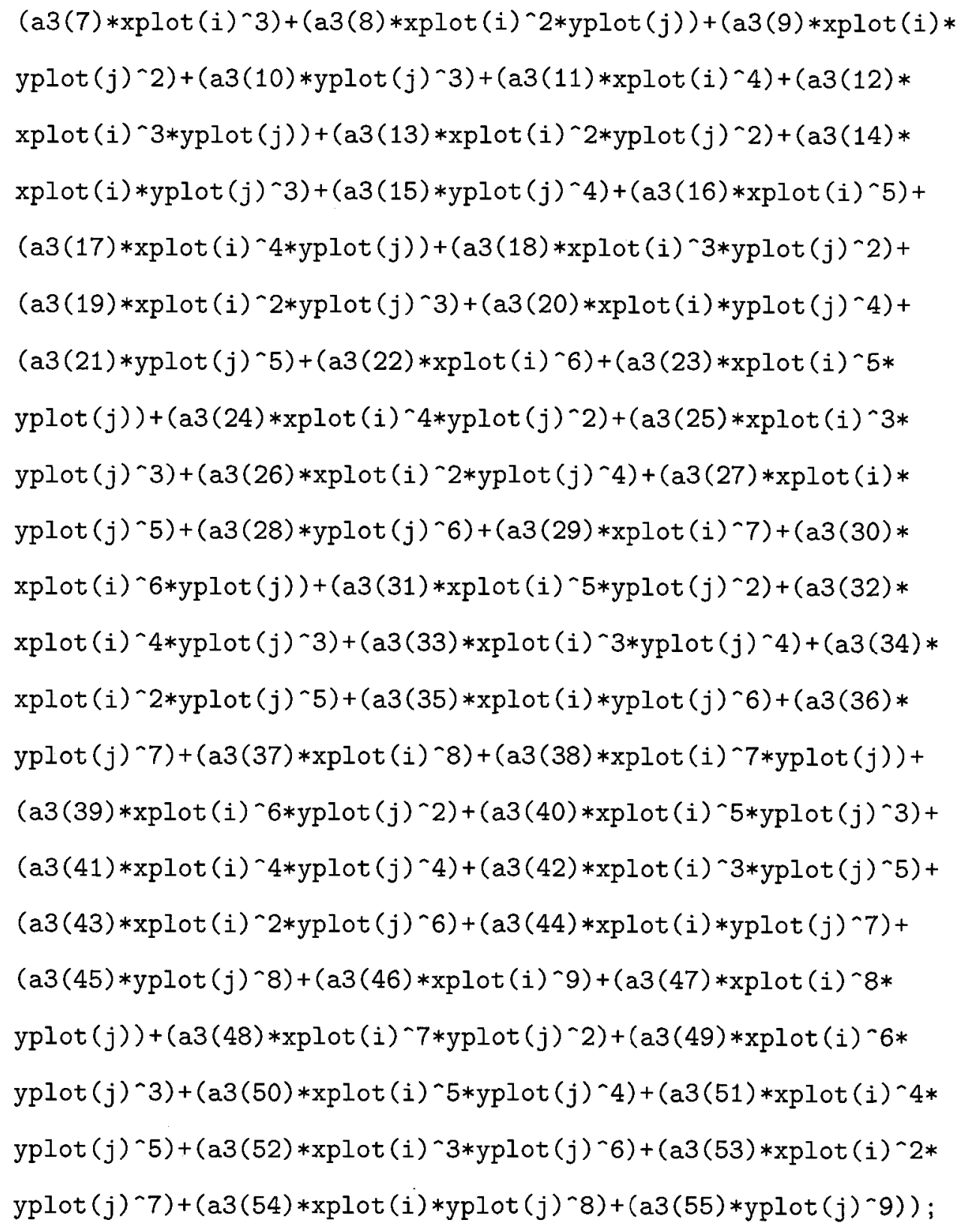


end

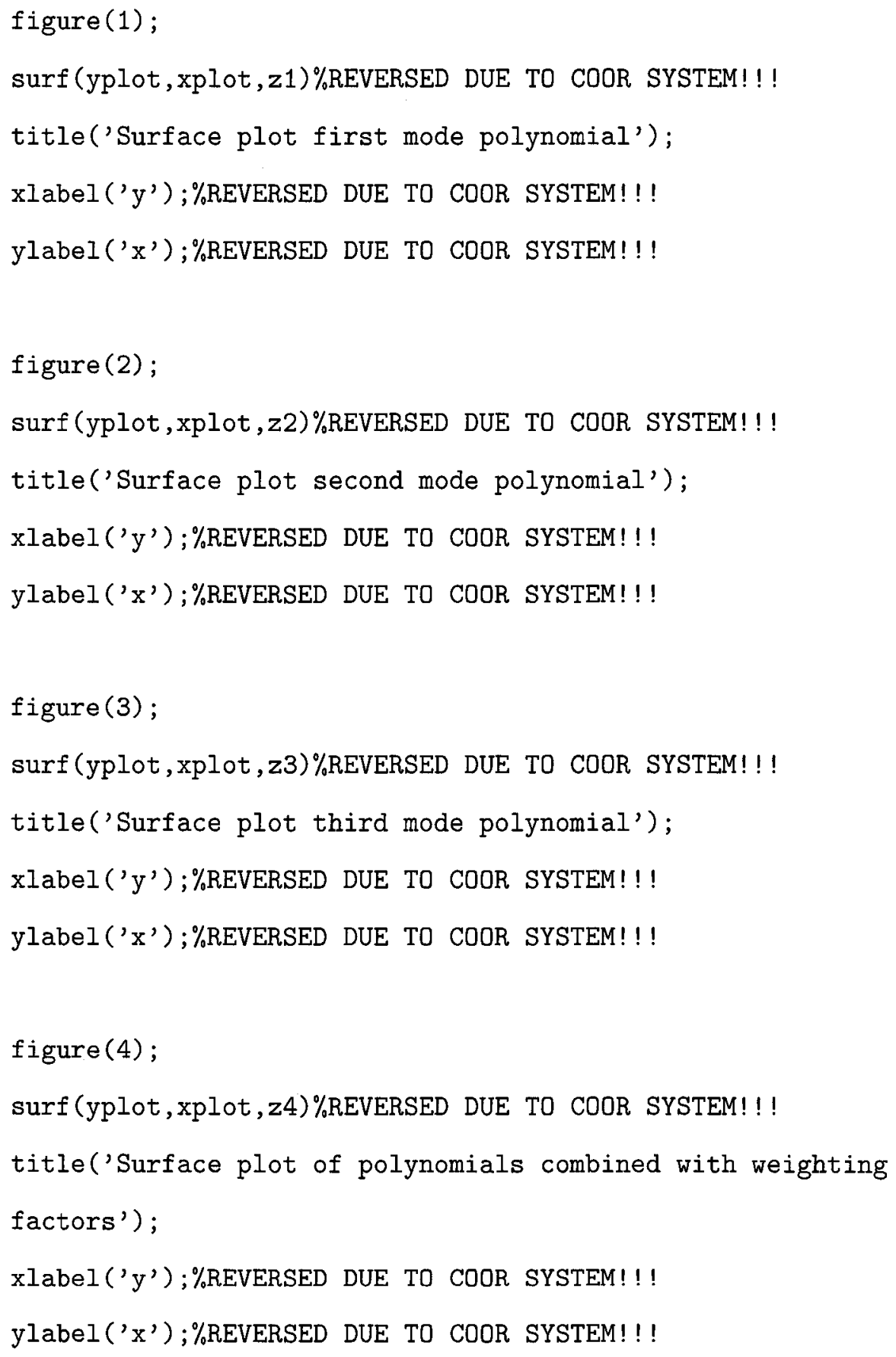




\section{Appendix $\mathrm{C}$}

\section{Grouping Optimization Matlab Code}

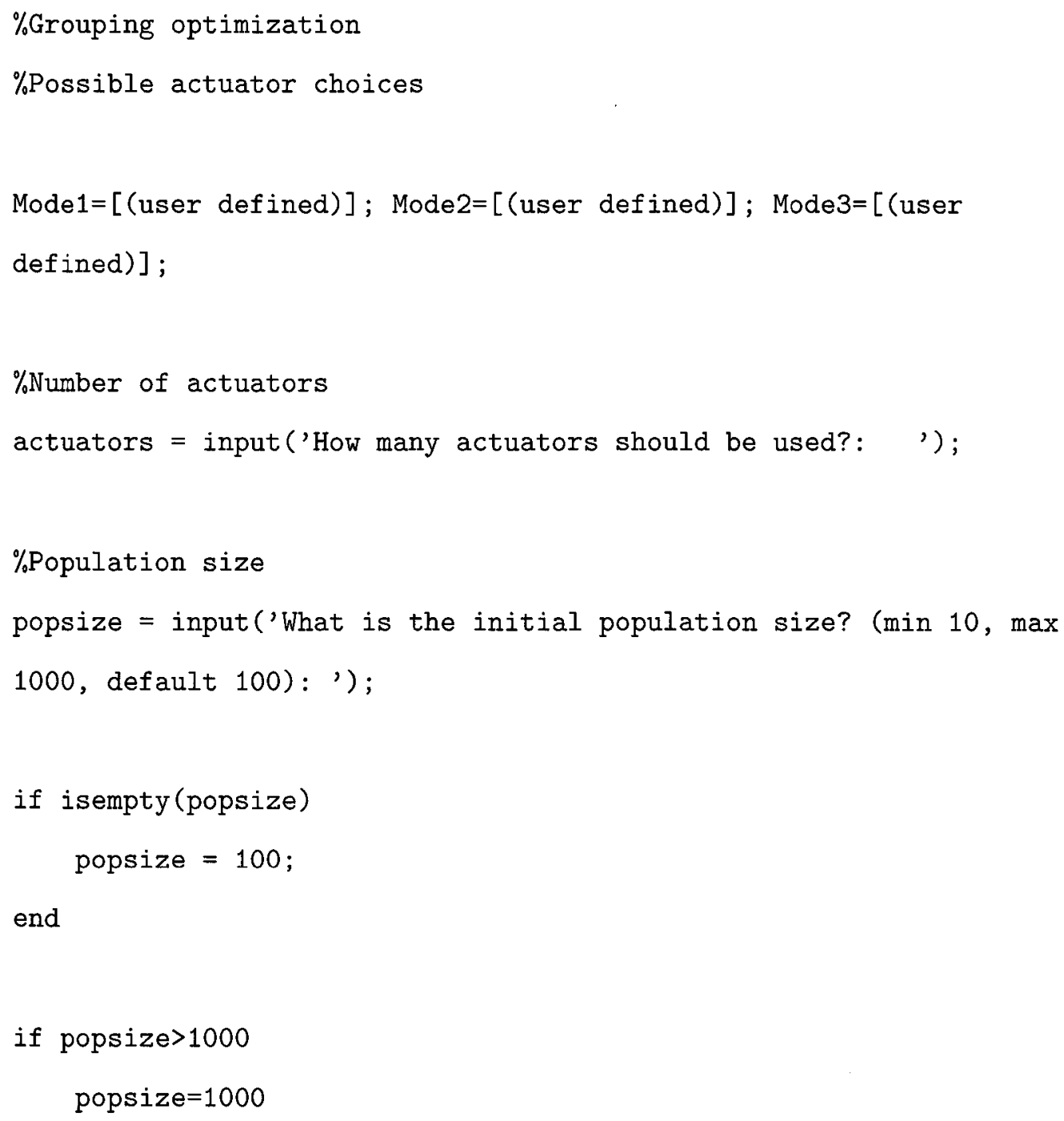


end

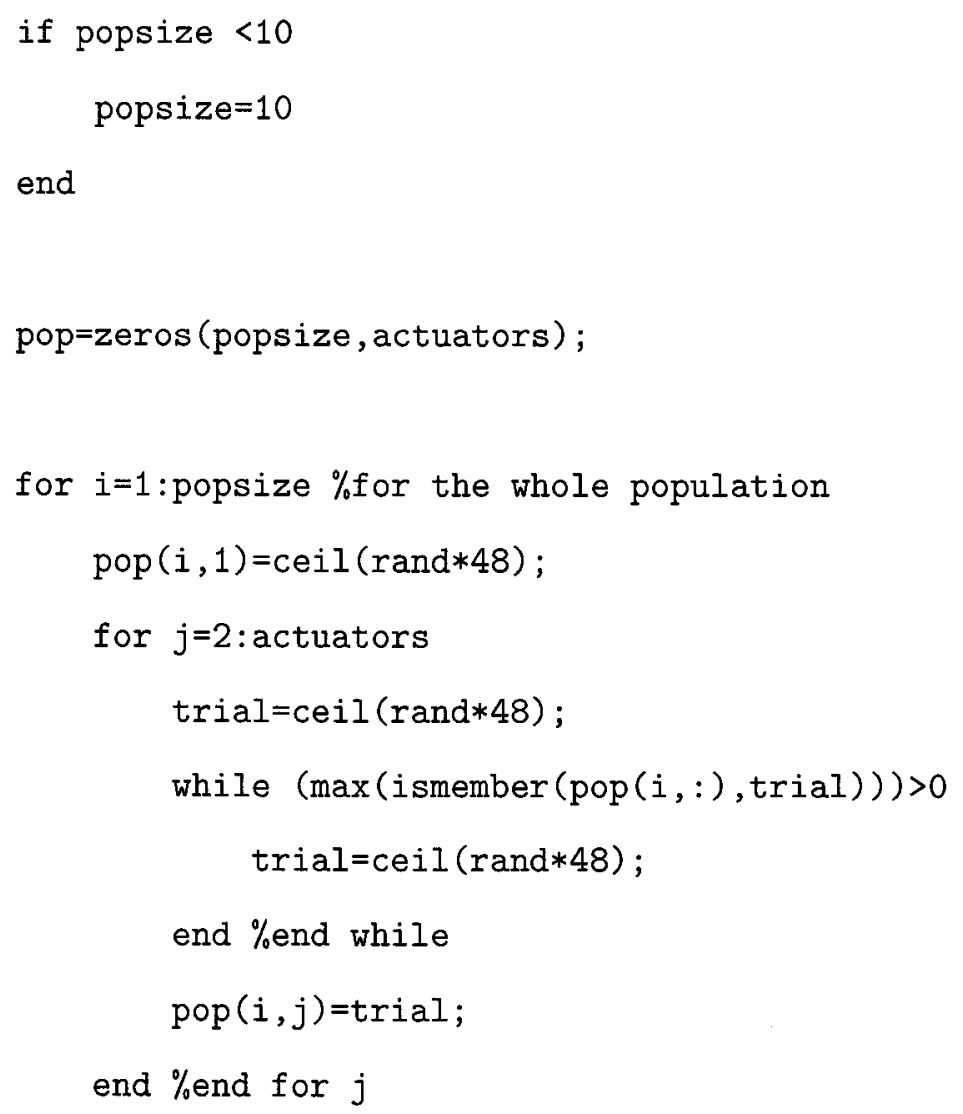

end \%end for $i$

$\%$ weighting factor 1

w1 = input ('What weighting factor on mode 1? (min 0, $\max 10$, default 10): ');

if isempty (w1) 


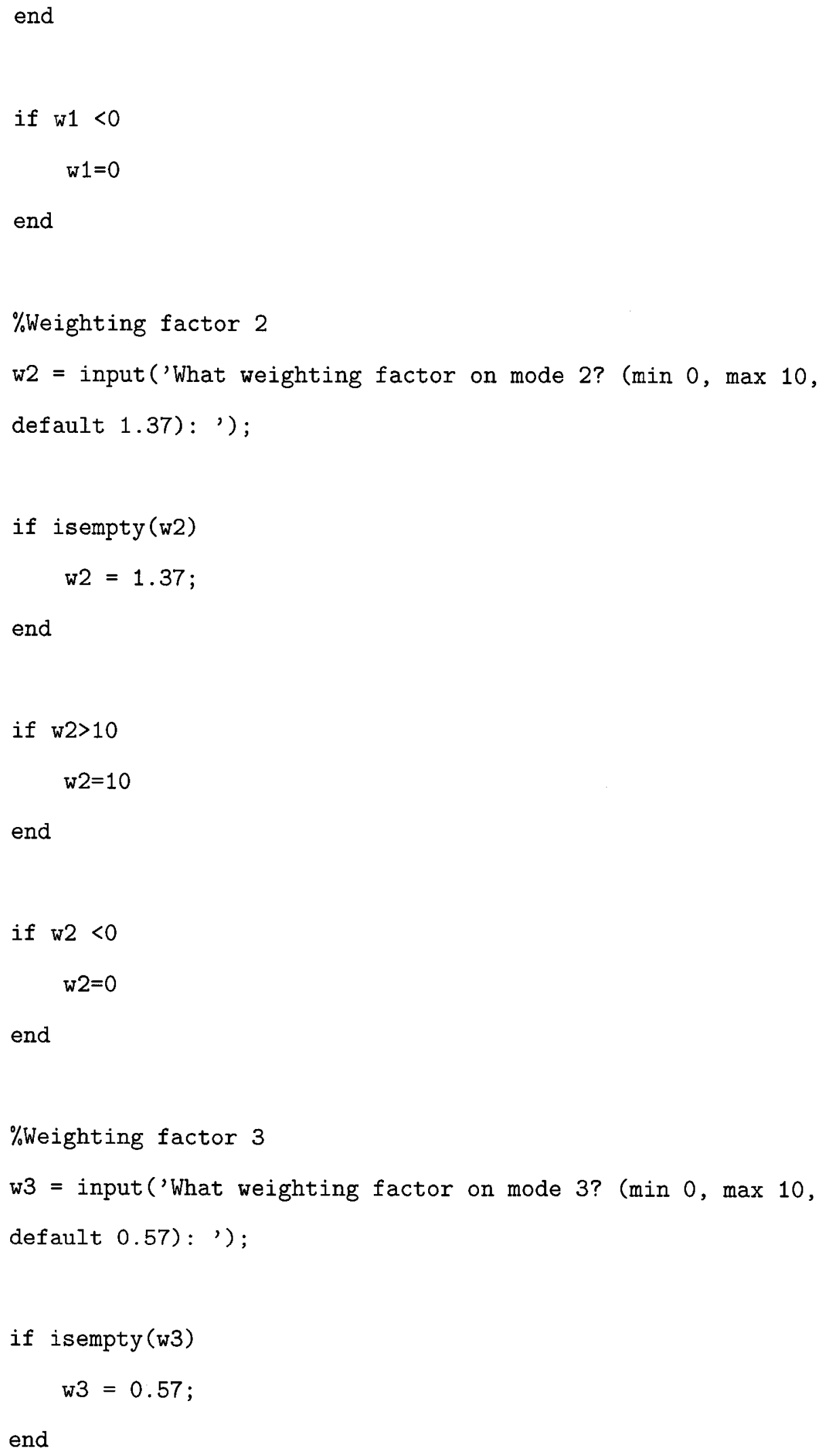




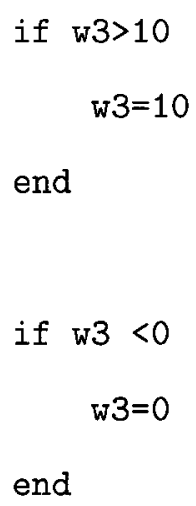




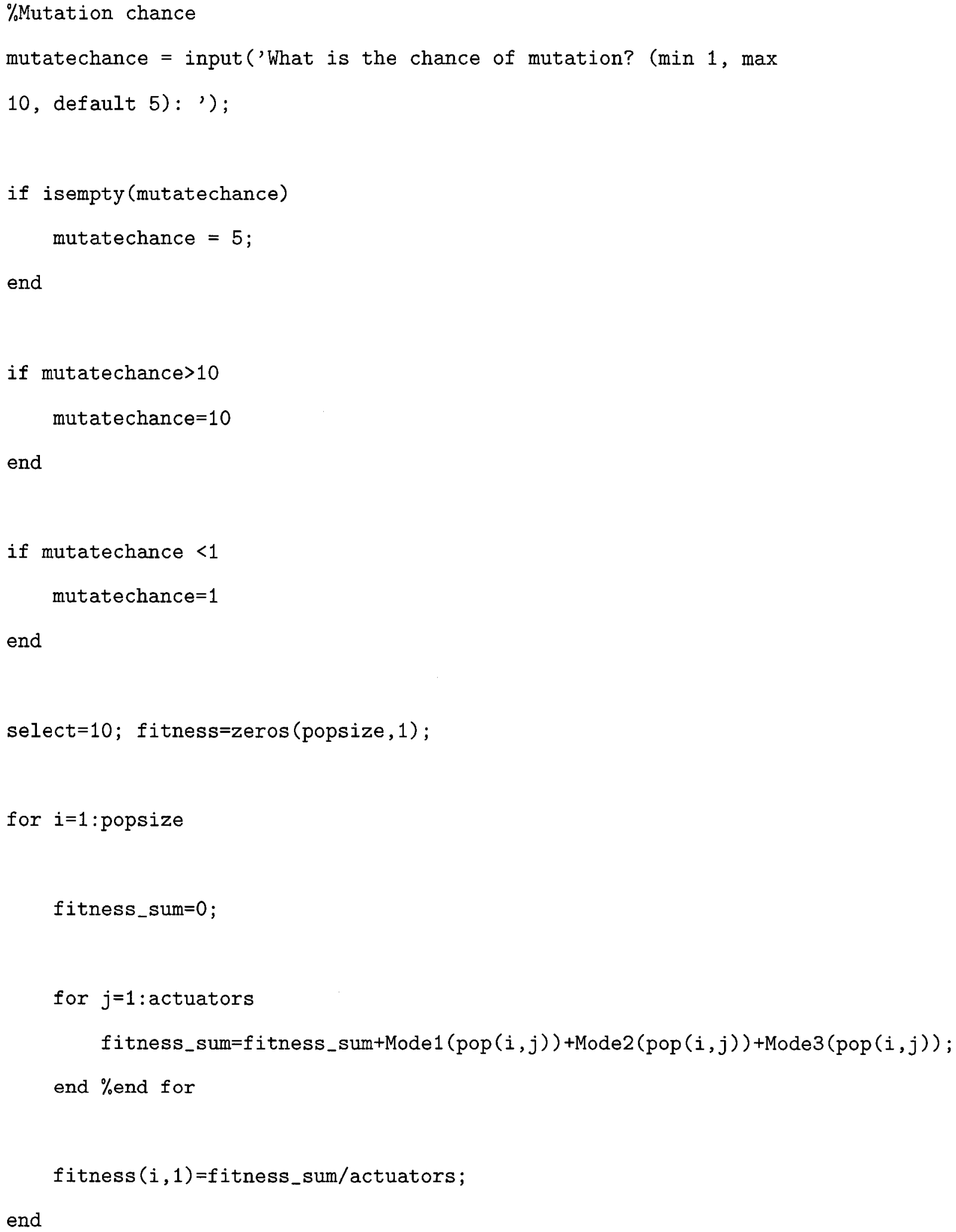


maximum_average_fitness=max(fitness) \%display the current best average fitness

fitnessplot (1) =max (fitness) ;

selection=zeros $($ select,1);

for $i=1$ :select $\%$ for the number of fitest individuals we wish to select $[C, I]=\max$ (fitness); \% find the index of the fitest individual selection(i)=I; \%store that index in the matrix selection fitness $(I)=0$; $\%$ set fitness of that individual to zero so the loop will select the next fitest, etc.

end \%end for i

\%Make new population by breeding all posible combinations. Note that the $\%$ ten parents are part of the population (self-cross), although they are $\%$ subject to mutation (if favourable)

newpop=zeros (select`2, actuators); \%declare newpopulation matrix. Note for breeding all selected values, the number of possible $\%$ combinations is select` 2 . generation $=0$

while generation<maxgen newpop=zeros (select^2, actuators); \%declare newpopulation matrix. Note for breeding all selected values, the number of possible $\%$ combinations is select` 2 . 
generation=generation $+1 \%$ Increase number of generations by 1 .

for $r=1$ :select \%for each of the selected individuals primarycross=selection( $r$ ); \%set each one in turn as the primary cross individual

for $i=1$ : select $\%$ for each other selected individual (including itself for self cross). This is the secondary cross.

level $=(r-1) *$ select $+i ; \%$ find position in newpopulation matrix based on the primary and secondary cross.

for $j=1$ :actuators $\%$ for every actuator alpha=round (rand); \%result of cross; 0 or 1 on primary vs. secondary cross. trial=round (alpha*pop (primarycross,$j)+(1-a l p h a) * \operatorname{pop}(\operatorname{selection}(i), j))$; $\%$ set allele to primary or secondary cross and round to ensure integer if $(\max ($ ismember (newpop (level, : ), trial)) )>0 trial=round $((1-a l p h a) *$ pop (primarycross,$j)+($ alpha $) *$ $\operatorname{pop}(\operatorname{selection}(i), j))$;

if (max (ismember (newpop (level, :), trial))) >0 trial=ceil (rand $* 48) ; \%$ forced mutation while (max (ismember (newpop(level, :), trial))) $>0$ trial=ceil $(\operatorname{rand} * 48)$; end \%end while end $\%$ end if end $\%$ end if newpop $($ level,$j)=\operatorname{trial}$;

end \%end for $j$

end $\%$ end for $i$

end \%end for $r$ 


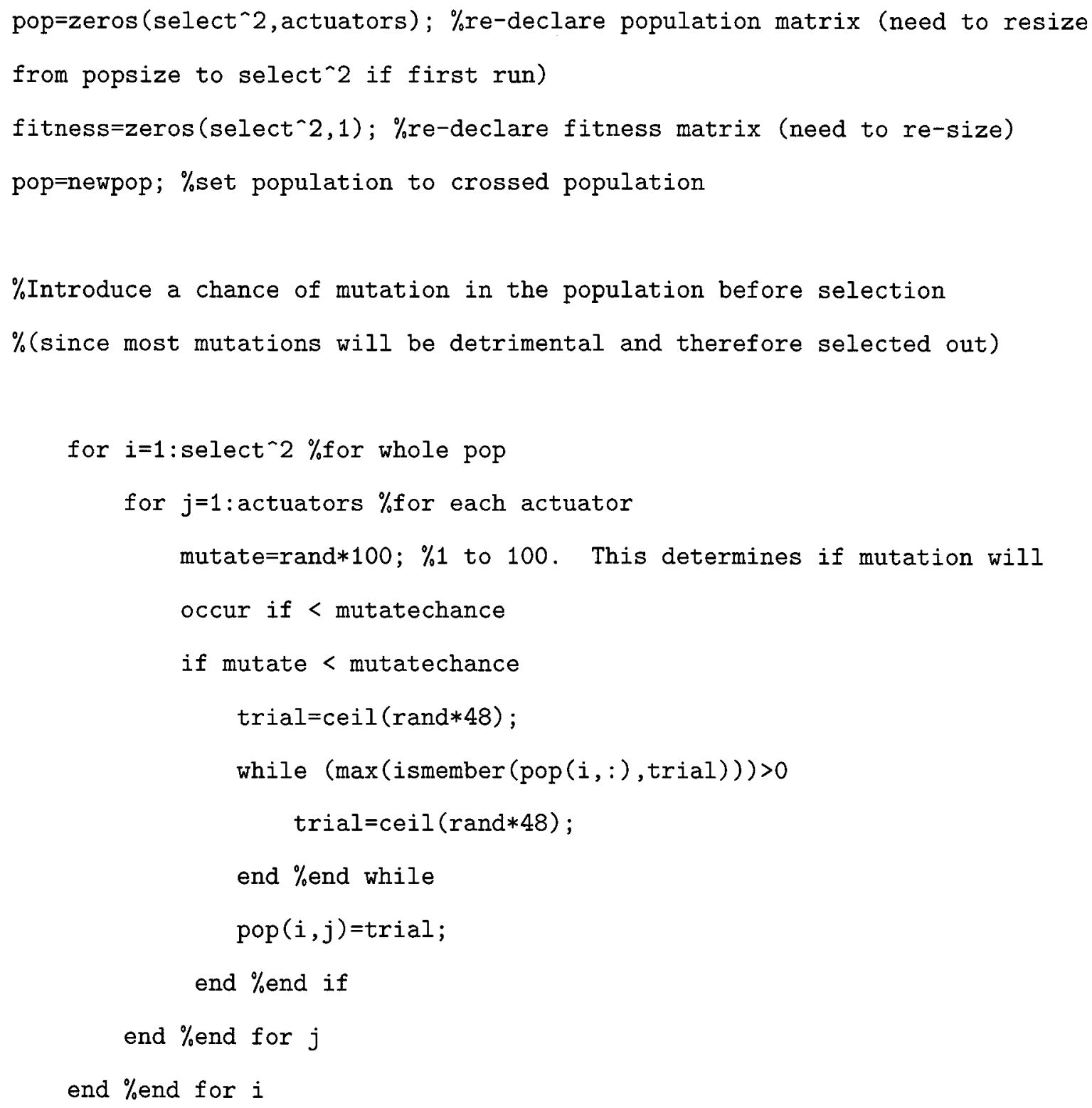


for $j=1$ :actuators

fitness_sum=fitness_sum+Mode1 $(\operatorname{pop}(i, j))+\operatorname{Mode} 2(\operatorname{pop}(i, j))+\operatorname{Mode} 3(\operatorname{pop}(i, j))$; end $\%$ end for $j$

fitness $(i, 1)=f i t n e s s_{\text {sum/actuators }}$;

end $\%$ end for $i$

maximum_average_fitness=max (fitness) \%display the current best average fitness

fitnessplot (1)=max (fitness);

selection=zeros (select, 1$)$;

for $i=1:$ select $\%$ for the number of fitest individuals we wish to select

$[C, I]=\max (f i t n e s s) ; \%$ find the index of the fitest individual

selection(i)=I; \%store that index in the matrix selection

fitness(I) $=0$; \%set fitness of that individual to zero so the loop will

select the next fitest, etc.

end \%end for $i$

end \%end of algorith while loop

$\%$ plot fitness over generations

figure(1); plot (fitnessplot); xlabel('Generation');

ylabel('Fitness'); title('Fitness vs. Generation Plot');

$\%$ display best set of actuators

best_actuator_set=sort $(\operatorname{pop}(\operatorname{selection}(1),:))$ 